Supporting Information

\title{
Modular Approach to Substituted Pyridoazepinones
}

\author{
Valentin S. Dorokhov and Samir Z. Zard* \\ Laboratoire de Synthèse Organique, CNRS UMR 7652, Ecole Polytechnique, \\ 91128 Palaiseau Cedex, France \\ *samir.zard@polytechnique.edu
}

\section{Table of contents}

General experimental methods S2

Synthesis of starting xanthates S3

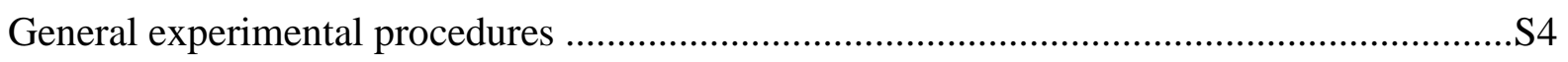

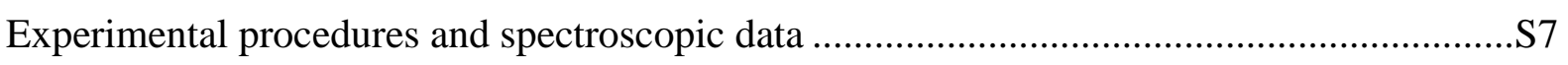

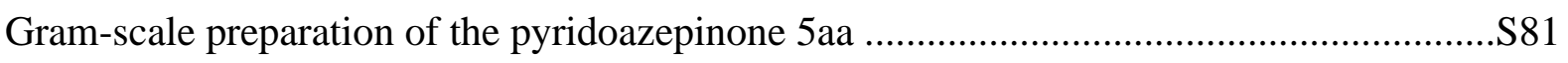

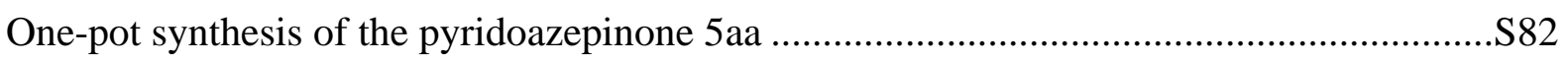

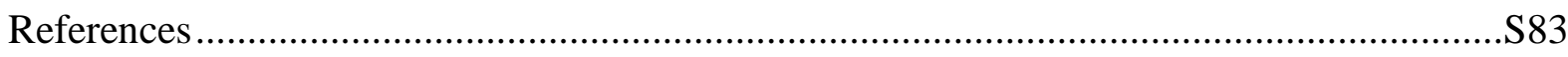

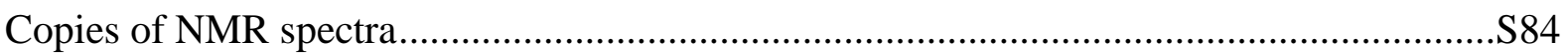




\section{General experimental methods}

Reactions sensitive to air and/or moisture were performed under nitrogen atmosphere. For the reactions that require heating, an oil bath or a DrySyn heating block was used as the heat source.

Flash column chromatography was carried out on silica gel $60(40-63 \mu \mathrm{m})$. Thin layer chromatography (TLC) was performed on alumina plates precoated with silica gel (Merck silica gel, $\left.60 \mathrm{~F}_{254}\right)$. Products spots were visualized by exposure to the UV light $\left(\lambda_{\max }=254 \mathrm{~nm}\right)$ and/or by staining with vanillin in acidic ethanol solution and/or basic aqueous $\mathrm{KMnO}_{4}$, followed by heating. Preparative TLC was performed on glass plates coated with silica gel (Merck PLC silica gel, $60 \mathrm{~F}_{254}$ ). Petroleum ether refers to the fraction of petroleum boiling between $40^{\circ} \mathrm{C}$ and $60^{\circ} \mathrm{C}$.

Nuclear magnetic resonance spectra were recorded at ambient temperature on a Bruker Avance DPX 400 instrument. Proton magnetic resonance spectra ( ${ }^{1} \mathrm{H}$ NMR) were recorded at $400 \mathrm{MHz}$ and coupling constants $(\mathrm{J})$ are reported to $\pm 0.5 \mathrm{~Hz}$. The following abbreviations were utilized to describe peak patterns when appropriate: $\mathrm{br}=$ broad, $\mathrm{s}=$ singlet, $\mathrm{d}=$ doublet, $\mathrm{t}=$ triplet, $\mathrm{q}=$ quartet and $\mathrm{m}=$ multiplet. Carbon magnetic resonance spectra $\left({ }^{13} \mathrm{C} \mathrm{NMR}\right)$ were recorded at $101 \mathrm{MHz}$. Chemical shifts (H, C) are quoted in parts per million (ppm) and are referenced to the residual solvent peak $\left(\mathrm{CDCl}_{3}: \delta \mathrm{H}=7.26\right.$ and $\delta \mathrm{C}=77.2$; DMSO: $\delta \mathrm{H}=2.50$ and $\delta \mathrm{C}=39.5$; Acetone: $\delta \mathrm{H}=2.05$ and $\delta \mathrm{C}=29.8$ ).

High resolution mass spectrometry experiments were performed on a tims-TOF mass spectrometer (Bruker, France). Electrospray source has been used in positive mode. The quoted masses are accurate to $\pm 5 \mathrm{ppm}$. Infrared spectra were recorded on a Perkin-Elmer Spectrum Two apparatus. Absorption maxima $\left(v_{\max }\right)$ are reported in wavenumbers $\left(\mathrm{cm}^{-1}\right)$. Melting points were recorded in degrees Celsius $\left({ }^{\circ} \mathrm{C}\right)$, using a Stuart SMP40 Automatic Melting Point apparatus. 


\section{Synthesis of starting xanthates}

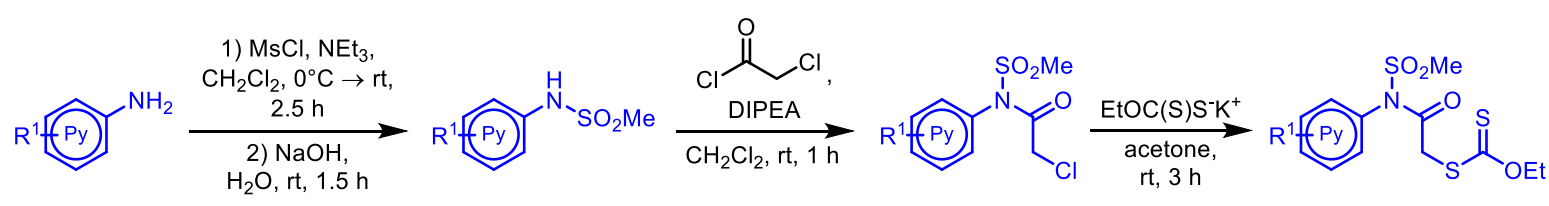

2-R $R^{1}-5$-amino:

$\mathrm{R}^{1}=\mathrm{Br}, \mathrm{R}^{2}=\mathrm{H}$

1b $(99 \%)$

2b $(76 \%)$

3b (82\%)

$\mathrm{R}^{1}=\mathrm{CH}_{3}, \mathrm{R}^{2}=\mathrm{H}$

1c $(65 \%)$

2c $(59 \%)$

3c $(80 \%)$

$\mathrm{R}^{1}=\mathrm{CF}_{3}, \mathrm{R}^{2}=\mathrm{H}$

1d $(98 \%)$

2d $(73 \%)$

3d $(80 \%)$

$\mathrm{R}^{1}=\mathrm{Cl}, \mathrm{R}^{2}=\mathrm{CH}_{3}$

2 e $(57 \%)$

$3 \mathbf{e}(72 \%)$

\section{2- $\boldsymbol{R}^{\mathbf{1}-4-a m i n o:}$}

$\mathrm{R}^{1}=\mathrm{Cl}, \mathrm{R}^{2}=\mathrm{H}$

$1 f(65 \%)$

2 f $(55 \%)$

$3 f(71 \%)$

2-R ${ }^{1}-6$-amino:

$\mathrm{R}^{1}=\mathrm{Cl}, \mathrm{R}^{2}=\mathrm{H}$

1g $(87 \%)$

2g $(77 \%)$

$3 g(63 \%)$<smiles>CCOC(=S)SCC(=O)N(c1ccc(Br)nc1)S(C)(=O)=O</smiles>

3b<smiles>CCOC(=S)SCC(=O)N(c1ccc(C)nc1)S(C)(=O)=O</smiles>

$3 c$<smiles>CCOC(=S)SCC(=O)N(c1ccc(C(F)(F)F)nc1)S(C)(=O)=O</smiles>

$3 d$<smiles>CCOC(=S)SC(C)C(=O)N(c1ccc(Cl)nc1)S(C)(=O)=O</smiles><smiles>CCOC(=S)SCC(=O)N(c1ccnc(Cl)c1)S(C)(=O)=O</smiles>

$3 f$<smiles>CCOC(=S)SCC(=O)N(c1cccc(Cl)n1)[N+](=O)[O-]</smiles>

$3 \mathrm{~g}$ 


\section{General experimental procedures}

\section{General procedure A (Sulfonylation of aminopyridines)}

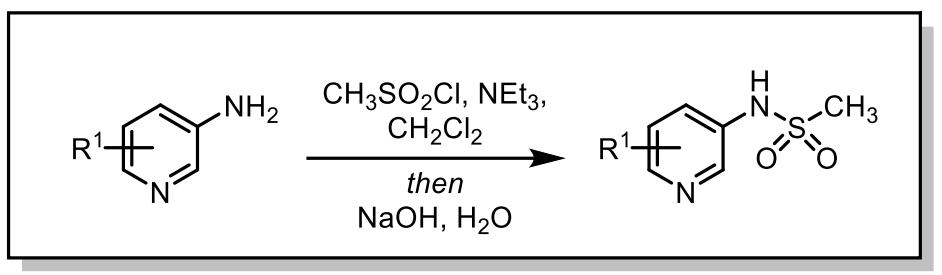

According to a modified literature procedure ${ }^{1}$, aminopyridine (1 eq.) was dissolved in DCM (1 M solution), then $\mathrm{Et}_{3} \mathrm{~N}$ (2.25 eq.) was added. The reaction mixture was cooled to $0^{\circ} \mathrm{C}$, and a solution of methanesulfonyl chloride (2.25 eq.) in DCM was added dropwise under $\mathrm{N}_{2}$ atmosphere. Reaction mixture was stirred at r.t. for 2.5-4 h, then concentrated under reduced pressure. A 2.5 M aqueous solution of $\mathrm{NaOH}$ (10 eq.) was added, and the reaction mixture was stirred for 1.5-4 h (until all solid material dissolved). The mixture was then diluted with $2.0 \mathrm{M}$ $\mathrm{HCl}$ aqueous solution (ca. 10 eq.) to ensure the acidic $\mathrm{pH}$, and extracted with EtOAc. The extract was washed with brine, dried over $\mathrm{Na}_{2} \mathrm{SO}_{4}$ and concentrated under reduced pressure to afford target product, which could be further recrystallized or used directly for the next step.

\section{General procedure B (Acylation of sulfonamide)}

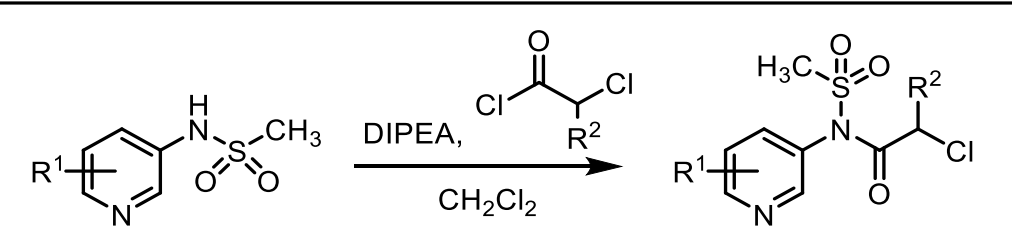

Sulfonamide (1 eq.) was mixed with DCM $(0.5 \mathrm{M})$, and the mixture was cooled to $0^{\circ} \mathrm{C}$. DIPEA (1.5 eq.) and chloroacetyl or chloropropionyl chloride (2.0 eq.) were added under $\mathrm{N}_{2}$ atmosphere, and the mixture was stirred at r.t. for 1-2 h. It was then diluted with DCM, and poured into sat. $\mathrm{NH}_{4} \mathrm{Cl}$ solution. The organic layer was washed with sat. $\mathrm{NH}_{4} \mathrm{Cl}$ solution and brine, dried over $\mathrm{Na}_{2} \mathrm{SO}_{4}$ and concentrated under reduced pressure. The crude material was recrystallized from EtOAc or purified by column chromatography to afford the target product. 


\section{General procedure C (Substitution of chlorine to xanthate)}

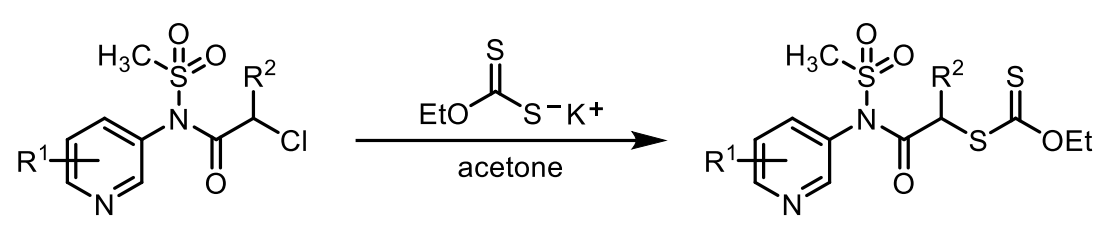

Chloride (1 eq.) was dissolved in acetone ( $0.3 \mathrm{M}$ solution with respect to chloride) at r.t., and potassium ethyl xanthogenate (1.05 eq.) was added. The reaction mixture was stirred at r.t. for 3-4 h, then concentrated under reduced pressure. The residue was dissolved in EtOAc and washed with brine, the organic layer was dried over $\mathrm{Na}_{2} \mathrm{SO}_{4}$ and concentrated under reduced pressure. The crude material was purified by flash column chromatography to afford the target product.

\section{General procedure D (Xanthate addition-transfer process)}

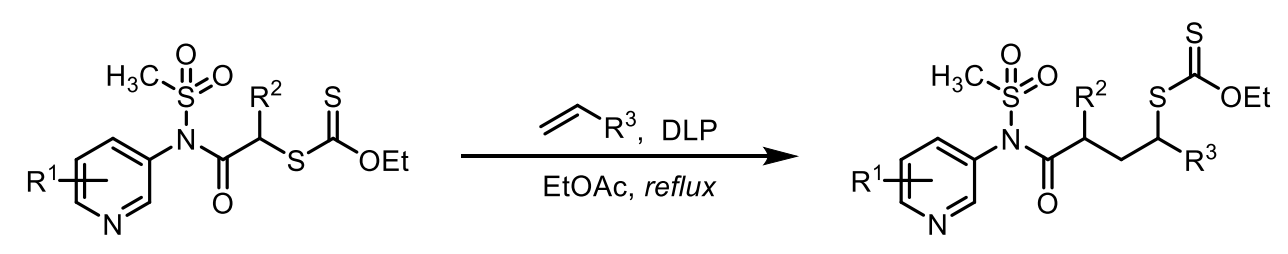

A stirred solution of the xanthate (1 eq.) and the olefin (1.1-2 eq.) in EtOAc (1.0 M of xanthate) was refluxed for 15-20 min under $\mathrm{N}_{2}$. Lauroyl peroxide (DLP) (0.1 eq.) was then added to the refluxing solution, followed by additional portions ( 0.1 eq.) every $60 \mathrm{~min}$ until the starting xanthate was completely consumed (analysis by TLC). The reaction mixture was then cooled to room temperature, concentrated under reduced pressure and purified by flash column chromatography on silica gel to yield the desired compounds.

\section{General procedure E (Radical cyclization)}

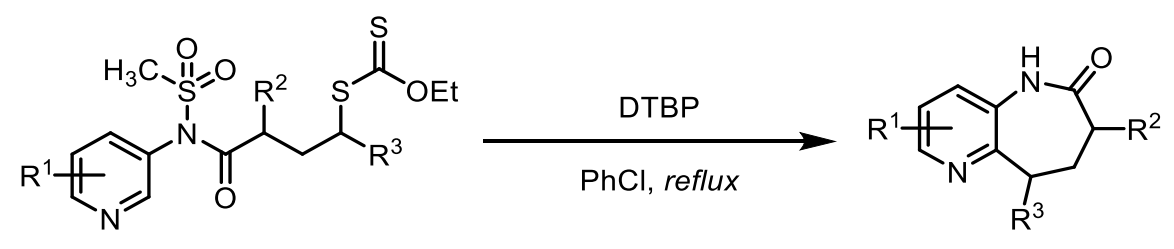

A stirred solution of xanthate (1 eq.) in chlorobenzene (0.01-0.02 $\mathrm{M}$ of xanthate) was refluxed for 15-20 min under $\mathrm{N}_{2}$. Di-tert-butylperoxide (DTBP) (5 eq.) was then added by portions to 
the refluxing solution. The reaction was monitored by TLC every hour until the starting xanthate was completely consumed. The reaction mixture was then cooled down to room temperature, concentrated under reduced pressure and purified by flash column chromatography on silica gel or recrystallized to yield the desired compounds. 


\section{Experimental procedures and spectroscopic data}

\section{N-(6-chloropyridin-3-yl)methanesulfonamide (1a)}<smiles>CS(=O)(=O)Nc1ccc(Cl)nc1</smiles>

Following the general procedure A, the reaction was carried out with the solution of 5-amino2-chloropyridine (10.0 g, $77.8 \mathrm{mmol}, 1$ eq.) in DCM (78 mL), Et ${ }_{3} \mathrm{~N}$ (24.0 mL, $175 \mathrm{mmol}, 2.25$ eq.), and a solution of methanesulfonyl chloride (13.6 mL, $175 \mathrm{mmol}, 2.25$ eq.) in DCM (39 $\mathrm{mL}$ ) during $2.5 \mathrm{~h}$. After concentration of the reaction mixture, the second step was carried out with $2.5 \mathrm{M}$ solution of $\mathrm{NaOH}(310 \mathrm{~mL})$ for $1.5 \mathrm{~h}$. It was followed by the addition of $2.0 \mathrm{M} \mathrm{HCl}$ solution $(390 \mathrm{~mL})$, and extraction with EtOAc $(3 \times 200 \mathrm{~mL})$. Concentration of the solution under reduced pressure afforded crude target material (15.8 g). Recrystallization from $\mathrm{PE} / \mathrm{EtOAc}$ in freezer afforded pure target product $\mathbf{1 a}(13.5 \mathrm{~g}, 84 \%)$ as light brown crystals.

${ }^{1} \mathrm{H}$ NMR (400 MHz, DMSO- $\left.d_{6}\right) \delta 10.18(\mathrm{~s}, 1 \mathrm{H}, \mathrm{NH}), 8.24\left(\mathrm{dd}, J=2.9,0.7 \mathrm{~Hz}, 1 \mathrm{H}, \mathrm{CH}_{\mathrm{Ar}}\right), 7.67$ $\left(\mathrm{dd}, J=8.6,2.9 \mathrm{~Hz}, 1 \mathrm{H}, \mathrm{CH}_{\mathrm{Ar}}\right), 7.50\left(\mathrm{dd}, J=8.6,0.7 \mathrm{~Hz}, 1 \mathrm{H}, \mathrm{CH}_{\mathrm{Ar}}\right), 3.09\left(\mathrm{~s}, 3 \mathrm{H}, \mathrm{SO}_{2} \mathrm{CH}_{3}\right)$. ${ }^{13} \mathrm{C}$ NMR $\left(101 \mathrm{MHz}, \mathrm{DMSO}-d_{6}\right) \delta 144.7\left(\mathrm{C}_{\mathrm{Ar}}\right), 141.0\left(\mathrm{CH}_{\mathrm{Ar}}\right), 134.8\left(\mathrm{C}_{\mathrm{Ar}}\right), 130.6\left(\mathrm{CH}_{\mathrm{Ar}}\right), 124.7$ $\left(\mathrm{CH}_{\mathrm{Ar}}\right), 39.8\left(\mathrm{SO}_{2} \mathrm{CH}_{3}\right)$.

IR (neat), $v\left(\mathrm{~cm}^{-1}\right): 3095,3026,2778,1578,1463,1371,1321,1260,1144,1105,965,836$. HRMS (ESI) m/z: [M + H] $]^{+}$Calcd for $\mathrm{C}_{6} \mathrm{H}_{8} \mathrm{ClN}_{2} \mathrm{O}_{2} \mathrm{~S}$ 206.9990; Found 206.9992.

$\mathrm{R}_{\mathrm{f}}=0.4(\mathrm{PE} / \mathrm{EtOAc}=50 \%)$.

m.p. $=132-134^{\circ} \mathrm{C}($ crystallized from PE/EtOAc $)$. 
<smiles>CS(=O)(=O)Nc1ccc(Br)nc1</smiles>

Following the general procedure A, the reaction was carried out with the solution of 2-amino5-bromopyridine (2.00 g, $11.6 \mathrm{mmol}, 1$ eq.) in DCM (12 mL), Et $3 \mathrm{~N}$ (3.70 mL, $26.0 \mathrm{mmol}, 2.25$ eq.), and a solution of methanesulfonyl chloride (2.00 mL, $26.0 \mathrm{mmol}, 2.25$ eq.) in DCM (6 $\mathrm{mL}$ ) during $3 \mathrm{~h}$. After concentration of the reaction mixture, the second step was carried out with $2.5 \mathrm{M}$ solution of $\mathrm{NaOH}(46 \mathrm{~mL})$ for $4 \mathrm{~h}$. It was followed by addition of $2.0 \mathrm{M} \mathrm{HCl}$ solution $(58 \mathrm{~mL})$, and extraction with EtOAc $(3 \times 50 \mathrm{~mL})$. Concentration of the solution under reduced pressure afforded target material $(2.86 \mathrm{~g}, 99 \%)$ as light brown crystals, sufficiently pure for the next step.

${ }^{1} \mathrm{H}$ NMR (400 MHz, DMSO- $\left.d_{6}\right) \delta 10.18(\mathrm{~s}, 1 \mathrm{H}, \mathrm{NH}), 8.23\left(\mathrm{dd}, J=2.8,0.8 \mathrm{~Hz}, 1 \mathrm{H}, \mathrm{CH}_{\mathrm{Ar}}\right), 7.62$ (dd, $\left.J=8.6,0.8 \mathrm{~Hz}, 1 \mathrm{H}, \mathrm{CH}_{\mathrm{Ar}}\right), 7.57$ (dd, $\left.J=8.6,2.8 \mathrm{~Hz}, 1 \mathrm{H}, \mathrm{CH}_{\mathrm{Ar}}\right), 3.09$ (s, $3 \mathrm{H}, \mathrm{SO}_{2} \mathrm{CH}_{3}$ ).

${ }^{13} \mathrm{C}$ NMR $\left(101 \mathrm{MHz}, \mathrm{DMSO}-d_{6}\right) \delta 141.5\left(\mathrm{CH}_{\mathrm{Ar}}\right), 135.2\left(\mathrm{C}_{\mathrm{Ar}}\right), 135.0\left(\mathrm{C}_{\mathrm{Ar}}\right), 130.3\left(\mathrm{CH}_{\mathrm{Ar}}\right), 128.4$ $\left(\mathrm{CH}_{\mathrm{Ar}}\right), 39.9\left(\mathrm{SO}_{2} \mathrm{CH}_{3}\right)$.

IR (neat), $v\left(\mathrm{~cm}^{-1}\right):$ 2897, 1573, 1486, 1458, 1367, 1318, 1257, 1144, 1093, 964, 920, 836.

HRMS (ESI) m/z: $[\mathrm{M}+\mathrm{H}]^{+}$Calcd for $\mathrm{C}_{6} \mathrm{H}_{8} \mathrm{BrN}_{2} \mathrm{O}_{2} \mathrm{~S}$ 250.9484; Found 250.9495.

$\mathrm{R}_{\mathrm{f}}=0.37(\mathrm{PE} / \mathrm{EtOAc}=50 \%)$.

m.p. $=150-151^{\circ} \mathrm{C}$. 
<smiles>Cc1ccc(NS(C)(=O)=O)cn1</smiles>

Following the general procedure A, the reaction was carried out with the solution of 5-amino2-methylpyridine (2.00 g, $18.5 \mathrm{mmol}, 1$ eq.) in DCM (18 mL), Et $3 \mathrm{~N}$ (5.80 mL, $41.6 \mathrm{mmol}, 2.25$ eq.), and a solution of methanesulfonyl chloride (3.20 mL, $41.6 \mathrm{mmol}, 2.25$ eq.) in DCM (9 $\mathrm{mL}$ ) during $3 \mathrm{~h}$. After concentration of the reaction mixture, the second step was carried out with $2.5 \mathrm{M}$ solution of $\mathrm{NaOH}(74 \mathrm{~mL})$ for $4 \mathrm{~h}$. It was followed by addition of $2.0 \mathrm{M} \mathrm{HCl}$ solution $(92 \mathrm{~mL})$. Then the mixture was neutralized with saturated $\mathrm{NaHCO}_{3}$ solution $(150 \mathrm{~mL})$ to $\mathrm{pH} \approx 7-8$, and extracted with EtOAc $(4 \times 50 \mathrm{~mL})$. Concentration of the solution under reduced pressure afforded target material $(2.24 \mathrm{~g}, 65 \%)$ as yellowish crystals, sufficiently pure for the next step.

${ }^{1} \mathrm{H}$ NMR (400 MHz, DMSO- $\left.d_{6}\right) \delta 9.79$ (s, $\left.1 \mathrm{H}, \mathrm{NH}\right), 8.30\left(\mathrm{dd}, J=2.6,0.8 \mathrm{~Hz}, 1 \mathrm{H}, \mathrm{CH}_{\mathrm{Ar}}\right), 7.51$ (ddd, $J=8.4,2.6 \mathrm{~Hz}, 1 \mathrm{H}, \mathrm{CH}_{\mathrm{Ar}}$ ), 7.23 (dt, $J=8.4,0.8 \mathrm{~Hz}, 1 \mathrm{H}, \mathrm{CH}_{\mathrm{Ar}}$ ), $3.00\left(\mathrm{~s}, 3 \mathrm{H}, \mathrm{SO}_{2} \mathrm{CH}_{3}\right.$ ), $2.42\left(\mathrm{~s}, 3 \mathrm{H}, \mathrm{CH}_{3}\right)$.

${ }^{13} \mathrm{C}$ NMR $\left(101 \mathrm{MHz}, \mathrm{DMSO}-d_{6}\right) \delta 153.7\left(\mathrm{C}_{\mathrm{Ar}}\right), 141.5\left(\mathrm{CH}_{\mathrm{Ar}}\right), 132.4\left(\mathrm{C}_{\mathrm{Ar}}\right), 128.5\left(\mathrm{CH}_{\mathrm{Ar}}\right), 123.3$ $\left(\mathrm{CH}_{\mathrm{Ar}}\right), 39.4\left(\mathrm{SO}_{2} \mathrm{CH}_{3}\right), 23.3\left(\mathrm{CH}_{3}\right)$.

IR (neat), $v\left(\mathrm{~cm}^{-1}\right): 3010,1580,1492,1377,1315,1290,1262,1136,981,935,843,730$.

HRMS (ESI) m/z: [M + H] $]^{+}$Calcd for $\mathrm{C}_{7} \mathrm{H}_{11} \mathrm{~N}_{2} \mathrm{O}_{2} \mathrm{~S}$ 187.0536; Found 187.0544.

$\mathrm{R}_{\mathrm{f}}=0.28($ EtOAc)

m.p. $=169-172^{\circ} \mathrm{C}$. 


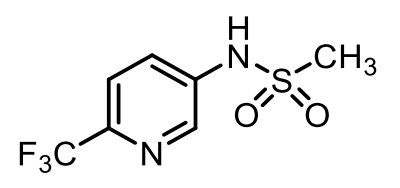

\section{Chemical Formula: $\mathrm{C}_{7} \mathrm{H}_{7} \mathrm{~F}_{3} \mathrm{~N}_{2} \mathrm{O}_{2} \mathrm{~S}$} Molecular Weight: 240.20

Following the general procedure A, the reaction was carried out with the solution of 5-amino2-(trifluoromethyl)pyridine (2.00 g, $12.3 \mathrm{mmol}, 1$ eq.) in DCM (12 mL), Et 3 N (3.90 mL, 27.8 mmol, 2.25 eq.), and a solution of methanesulfonyl chloride ( $2.20 \mathrm{~mL}, 27.8 \mathrm{mmol}, 2.25$ eq.) in DCM (6 mL) during $3.5 \mathrm{~h}$. After concentration of the reaction mixture, the second step was carried out with $2.5 \mathrm{M}$ solution of $\mathrm{NaOH}(50 \mathrm{~mL})$ for $3 \mathrm{~h}$. It was followed by addition of 2.0 $\mathrm{M} \mathrm{HCl}$ solution $(64 \mathrm{~mL})$, and extraction with EtOAc $(3 \times 50 \mathrm{~mL})$. Concentration of the solution under reduced pressure afforded target material $(2.91 \mathrm{~g}, 98 \%)$ as white crystals, sufficiently pure for the next step.

${ }^{1} \mathrm{H}$ NMR (400 MHz, DMSO- $\left.d_{6}\right) \delta 10.62(\mathrm{~s}, 1 \mathrm{H}, \mathrm{NH}), 8.55\left(\mathrm{dd}, J=2.6,1.0 \mathrm{~Hz}, 1 \mathrm{H}, \mathrm{CH}_{\mathrm{Ar}}\right), 7.88$ $\left(\mathrm{dd}, J=8.6,1.0 \mathrm{~Hz}, 1 \mathrm{H}, \mathrm{CH}_{\mathrm{Ar}}\right), 7.82\left(\mathrm{dd}, J=8.6,2.6 \mathrm{~Hz}, 1 \mathrm{H}, \mathrm{CH}_{\mathrm{Ar}}\right), 3.19\left(\mathrm{~s}, 3 \mathrm{H}, \mathrm{SO}_{2} \mathrm{CH}_{3}\right)$. ${ }^{13} \mathrm{C}$ NMR $\left(101 \mathrm{MHz}, \mathrm{DMSO}-d_{6}\right) \delta 140.6\left(\mathrm{C}-\mathrm{F},{ }^{2} J_{\mathrm{C}-F}=34.4 \mathrm{~Hz}, \mathrm{C}_{\mathrm{Ar}}\right), 140.3\left(\mathrm{CH}_{\mathrm{Ar}}\right), 138.3\left(\mathrm{C}_{\mathrm{Ar}}\right)$, $126.0\left(\mathrm{CH}_{\mathrm{Ar}}\right), 121.8\left(\mathrm{C}-\mathrm{F},{ }^{1} J_{\mathrm{C}-F}=273.2 \mathrm{~Hz}, \mathrm{CF}_{3}\right), 121.6\left(\mathrm{C}-\mathrm{F},{ }^{3} J_{\mathrm{C}-F}=2.9 \mathrm{~Hz}, \mathrm{CH}_{\mathrm{Ar}}\right), 40.3$ $\left(\mathrm{SO}_{2} \mathrm{CH}_{3}\right)$.

IR (neat), $v\left(\mathrm{~cm}^{-1}\right): 3221,1586,1473,1332,1317,1245,1130,1022,980,906,838,766$.

HRMS (ESI) m/z: $[\mathrm{M}+\mathrm{H}]^{+}$Calcd for $\mathrm{C}_{7} \mathrm{H}_{8} \mathrm{~F}_{3} \mathrm{~N}_{2} \mathrm{O}_{2} \mathrm{~S}$ 241.0253; Found 241.0263.

$\mathrm{R}_{\mathrm{f}}=0.47(\mathrm{PE} / \mathrm{EtOAc}=50 \%)$.

m.p. $=122-124^{\circ} \mathrm{C}$. 
<smiles>CS(=O)(=O)Nc1ccnc(Cl)c1</smiles>

Following the general procedure A, the reaction was carried out with the solution of 2chloropyridin-4-amine (2.50 g, $19.5 \mathrm{mmol}, 1$ eq.) in DCM (20 mL), Et $3 \mathrm{~N}$ (6.10 mL, $43.8 \mathrm{mmol}$, 2.25 eq.), and a solution of methanesulfonyl chloride ( $3.40 \mathrm{~mL}, 43.8 \mathrm{mmol}, 2.25$ eq.) in DCM $(10 \mathrm{~mL})$ during $2 \mathrm{~h}$. After concentration of the reaction mixture, the second step was carried out with $2.5 \mathrm{M}$ solution of $\mathrm{NaOH}(78 \mathrm{~mL})$ for $2 \mathrm{~h}$. It was followed by addition of $2.0 \mathrm{M} \mathrm{HCl}$ solution $(100 \mathrm{~mL})$, and extraction with EtOAc $(100 \mathrm{~mL}+2 \times 50 \mathrm{~mL})$. Concentration of the solution under reduced pressure and recrystallization from EtOAc afforded target material $(2.63 \mathrm{~g}, 65 \%)$ as white crystals.

${ }^{1} \mathrm{H}$ NMR (400 MHz, Acetone- $\left.d_{6}\right) \delta 9.50(\mathrm{~s}, 1 \mathrm{H}, \mathrm{NH}), 8.25\left(\mathrm{dd}, J=5.6,0.6 \mathrm{~Hz}, 1 \mathrm{H}, \mathrm{CH}_{\mathrm{Ar}}\right), 7.28$ $\left(\mathrm{dd}, J=2.0,0.5 \mathrm{~Hz}, 1 \mathrm{H}, \mathrm{CH}_{\mathrm{Ar}}\right), 7.23\left(\mathrm{dd}, J=5.6,2.0 \mathrm{~Hz}, 1 \mathrm{H}, \mathrm{CH}_{\mathrm{Ar}}\right), 3.24\left(\mathrm{~s}, 3 \mathrm{H}, \mathrm{SO}_{2} \mathrm{CH}_{3}\right)$.

${ }^{13} \mathrm{C}$ NMR $\left(101 \mathrm{MHz}\right.$, Acetone- $\left.d_{6}\right) \delta 152.9\left(\mathrm{C}_{\mathrm{Ar}}\right), 151.4\left(\mathrm{CH}_{\mathrm{Ar}}\right), 149.2\left(\mathrm{C}_{\mathrm{Ar}}\right), 112.2\left(\mathrm{CH}_{\mathrm{Ar}}\right), 112.2$ $\left(\mathrm{CH}_{\mathrm{Ar}}\right), 40.7\left(\mathrm{SO}_{2} \mathrm{CH}_{3}\right)$.

IR (neat), $v\left(\mathrm{~cm}^{-1}\right): 2761,1593,1491,1328,1233,1144,1087,1002,985,853,749$.

HRMS (ESI) m/z: [M + H] $]^{+}$Calcd for $\mathrm{C}_{6} \mathrm{H}_{8} \mathrm{ClN}_{2} \mathrm{O}_{2} \mathrm{~S}$ 206.9990; Found 206.9991.

$\mathrm{R}_{\mathrm{f}}=0.16(\mathrm{PE} / \mathrm{EtOAc}=40 \%)$.

m.p. $=144-146^{\circ} \mathrm{C}($ crystallized from EtOAc $)$. 
<smiles>CS(=O)(=O)Nc1cccc(Cl)n1</smiles>

Following the general procedure A, the reaction was carried out with the solution of 6chloropyridin-2-amine (2.50 g, $19.5 \mathrm{mmol}, 1$ eq.) in DCM (20 mL), Et $3 \mathrm{~N}$ (6.10 mL, $43.8 \mathrm{mmol}$, 2.25 eq.), and a solution of methanesulfonyl chloride (3.40 mL, $43.8 \mathrm{mmol}, 2.25$ eq.) in DCM $(10 \mathrm{~mL})$ during $2 \mathrm{~h}$. After concentration of the reaction mixture, the second step was carried out with $2.5 \mathrm{M}$ solution of $\mathrm{NaOH}(78 \mathrm{~mL})$ for $2 \mathrm{~h}$. It was followed by addition of $2.0 \mathrm{M} \mathrm{HCl}$ solution $(100 \mathrm{~mL})$, and extraction with EtOAc $(3 \times 100 \mathrm{~mL})$. Concentration of the solution under reduced pressure and purification by column chromatography (eluent: $\mathrm{PE} / \mathrm{EtOAc}=40 \%$ ) afforded target material $\mathbf{1 g}(3.50 \mathrm{~g}, 87 \%)$ as white crystals. The product was recrystallized from $\mathrm{Et}_{2} \mathrm{O}$ for analytical purposes.

${ }^{1} \mathrm{H}$ NMR (400 MHz, Chloroform- $d$ ) $\delta 8.11(\mathrm{~s}, 1 \mathrm{H}), 7.62\left(\mathrm{dd}, J=8.1,7.8 \mathrm{~Hz}, 1 \mathrm{H}, \mathrm{CH}_{\mathrm{Ar}}\right), 7.09$ $\left(\mathrm{dd}, J=8.1,0.7 \mathrm{~Hz}, 1 \mathrm{H}, \mathrm{CH}_{\mathrm{Ar}}\right), 7.04\left(\mathrm{dd}, J=7.8,0.7 \mathrm{~Hz}, 1 \mathrm{H}, \mathrm{CH}_{\mathrm{Ar}}\right), 3.29\left(\mathrm{~s}, 3 \mathrm{H}, \mathrm{SO}_{2} \mathrm{CH}_{3}\right)$.

${ }^{13} \mathrm{C}$ NMR $(101 \mathrm{MHz}$, Chloroform- $d) \delta 150.9\left(\mathrm{C}_{\mathrm{Ar}}\right), 149.9\left(\mathrm{C}_{\mathrm{Ar}}\right), 141.2\left(\mathrm{CH}_{\mathrm{Ar}}\right), 119.5\left(\mathrm{CH}_{\mathrm{Ar}}\right)$, $110.3\left(\mathrm{CH}_{\mathrm{Ar}}\right), 41.7\left(\mathrm{SO}_{2} \mathrm{CH}_{3}\right)$.

IR (neat), $v\left(\mathrm{~cm}^{-1}\right): 3240,1586,1570,1434,1376,1318,1142,969,938,881,787$.

HRMS (ESI) m/z: $[\mathrm{M}+\mathrm{H}]^{+}$Calcd for $\mathrm{C}_{6} \mathrm{H}_{8} \mathrm{ClN}_{2} \mathrm{O}_{2} \mathrm{~S}$ 206.9990; Found 206.9993.

$\mathrm{R}_{\mathrm{f}}=0.48(\mathrm{PE} / \mathrm{EtOAc}=40 \%)$.

m.p. $=98-100^{\circ} \mathrm{C}\left(\right.$ crystallized from $\left.\mathrm{Et}_{2} \mathrm{O}\right)$. 
<smiles>CS(=O)(=O)N(C(=O)CCl)c1ccc(Cl)nc1</smiles>

Following the general procedure $\mathrm{B}$, the reaction was carried out with sulfonamide $1 \mathrm{a}$ (13.2 $\mathrm{g}$, $63.9 \mathrm{mmol}, 1$ eq.) in DCM (128 mL), DIPEA (16.8 mL, $95.8 \mathrm{mmol}, 1.5$ eq.) and chloroacetyl chloride (10.2 mL, $127.8 \mathrm{mmol}, 2$ eq.) during 1 h. For work-up, DCM (200 mL) and sat. NH4Cl solution $(200 \mathrm{~mL}+100 \mathrm{~mL})$ were used. The crude material was recrystallized from EtOAc in freezer to afford the target product $\mathbf{2 a}$ as light brown crystals $(12.2 \mathrm{~g}, 67 \%)$.

${ }^{1} \mathrm{H}$ NMR (400 MHz, DMSO- $d_{6}$ ) $\delta 8.60\left(\mathrm{~d}, J=2.8 \mathrm{~Hz}, 1 \mathrm{H}, \mathrm{CH}_{\mathrm{Ar}}\right), 8.10(\mathrm{dd}, J=8.5,2.8 \mathrm{~Hz}$, $\left.1 \mathrm{H}, \mathrm{CH}_{\mathrm{Ar}}\right), 7.72\left(\mathrm{~d}, J=8.5 \mathrm{~Hz}, 1 \mathrm{H}, \mathrm{CH}_{\mathrm{Ar}}\right), 4.31\left(\mathrm{~s}, 2 \mathrm{H}, \mathrm{CH}_{2}\right), 3.64\left(\mathrm{~s}, 3 \mathrm{H}, \mathrm{SO}_{2} \mathrm{CH}_{3}\right)$.

${ }^{13} \mathrm{C}$ NMR $\left(101 \mathrm{MHz}, \mathrm{DMSO}-d_{6}\right) \delta 166.3(\mathrm{C}=\mathrm{O}), 151.5\left(\mathrm{C}_{\mathrm{Ar}}\right), 151.3\left(\mathrm{CH}_{\mathrm{Ar}}\right), 141.6\left(\mathrm{CH}_{\mathrm{Ar}}\right)$, $130.9\left(\mathrm{C}_{\mathrm{Ar}}\right), 125.3\left(\mathrm{CH}_{\mathrm{Ar}}\right), 44.8\left(\mathrm{CH}_{2}\right), 41.8\left(\mathrm{SO}_{2} \mathrm{CH}_{3}\right)$.

IR (neat), $v\left(\mathrm{~cm}^{-1}\right): 3043,1705,1566,1459,1360,1296,1235,1168,1109,1029,971,884$, $773,742$.

HRMS (ESI) m/z: $[\mathrm{M}+\mathrm{H}]^{+}$Calcd for $\mathrm{C}_{8} \mathrm{H}_{9} \mathrm{Cl}_{2} \mathrm{~N}_{2} \mathrm{O}_{3} \mathrm{~S}$ 282.9705; Found 282.9707.

$\mathrm{R}_{\mathrm{f}}=0.70(\mathrm{PE} / \mathrm{EtOAc}=50 \%)$.

m.p. $=152-156^{\circ} \mathrm{C}($ crystallized from EtOAc $)$. 
<smiles>CS(=O)(=O)N(C(=O)CCl)c1ccc(Br)nc1</smiles>

Following the general procedure $\mathrm{B}$, the reaction was carried out with sulfonamide $\mathbf{1 b}$ ( $2.68 \mathrm{~g}$, $10.7 \mathrm{mmol}, 1$ eq.) in DCM (21 mL), DIPEA (2.80 mL, $16.0 \mathrm{mmol}, 1.5$ eq.) and chloroacetyl chloride (1.70 mL, 21.4 mmol, 2 eq.) during 2 h. For work-up, DCM (30 mL) and sat. $\mathrm{NH}_{4} \mathrm{Cl}$ solution $(50 \mathrm{~mL}+30 \mathrm{~mL})$ were used. The crude material was recrystallized from EtOAc in freezer to afford the target product $\mathbf{2 b}$ as white crystals $(2.65 \mathrm{~g}, 76 \%)$.

${ }^{1} \mathrm{H}$ NMR (400 MHz, DMSO-d 6 ) $\delta 8.57\left(\mathrm{dd}, \mathrm{J}=2.8,0.7 \mathrm{~Hz}, 1 \mathrm{H}, \mathrm{CH}_{\mathrm{Ar}}\right.$ ), $7.98(\mathrm{dd}, \mathrm{J}=8.5,2.8$ $\left.\mathrm{Hz}, 1 \mathrm{H}, \mathrm{CH}_{\mathrm{Ar}}\right), 7.85\left(\mathrm{dd}, \mathrm{J}=8.4,0.7 \mathrm{~Hz}, 1 \mathrm{H}, \mathrm{CH}_{\mathrm{Ar}}\right), 4.31\left(\mathrm{~s}, 2 \mathrm{H}, \mathrm{CH}_{2}\right), 3.63\left(\mathrm{~s}, 3 \mathrm{H}, \mathrm{SO}_{2} \mathrm{CH}_{3}\right)$.

${ }^{13} \mathrm{C}$ NMR (101 MHz, DMSO-d 6$) \delta 166.2(\mathrm{C}=\mathrm{O}), 151.7\left(\mathrm{CH}_{\mathrm{Ar}}\right), 142.6\left(\mathrm{C}_{\mathrm{Ar}}\right), 141.4\left(\mathrm{CH}_{\mathrm{Ar}}\right)$, $131.3\left(\mathrm{C}_{\mathrm{Ar}}\right), 129.0\left(\mathrm{CH}_{\mathrm{Ar}}\right), 44.8\left(\mathrm{CH}_{2}\right), 41.8\left(\mathrm{SO}_{2} \mathrm{CH}_{3}\right)$.

IR (neat), $v\left(\mathrm{~cm}^{-1}\right): 3037,1704,1561,1454,1358,1296,1234,1167,1113,1090,969,882,70$, 736,665 .

HRMS (ESI) m/z: $[\mathrm{M}+\mathrm{H}]^{+}$Calcd for $\mathrm{C}_{8} \mathrm{H}_{9} \mathrm{BrClN}_{2} \mathrm{O}_{3} \mathrm{~S}$ 326.9200; Found 326.9215.

$\mathrm{R}_{\mathrm{f}}=0.60(\mathrm{PE} / \mathrm{EtOAc}=50 \%)$.

m.p. $=173-175^{\circ} \mathrm{C}($ crystallized from EtOAc $)$. 


\section{2-chloro-N-(6-methylpyridin-3-yl)-N-(methylsulfonyl)acetamide (2c)}<smiles>Cc1ccc(N(C(=O)CCl)S(C)(=O)=O)cn1</smiles>

Chemical Formula: $\mathrm{C}_{9} \mathrm{H}_{11} \mathrm{CIN}_{2} \mathrm{O}_{3} \mathrm{~S}$ Molecular Weight: 262.71

Following the general procedure $\mathrm{B}$, the reaction was carried out with sulfonamide $1 \mathrm{c}(2.07 \mathrm{~g}$, $11.1 \mathrm{mmol}, 1$ eq.) in DCM (22 mL), DIPEA (2.90 mL, $16.7 \mathrm{mmol}, 1.5$ eq.) and chloroacetyl chloride (1.80 mL, $22.2 \mathrm{mmol}, 2$ eq.) during 2 h. For work-up, DCM (30 mL) and sat. NH4Cl solution $(50 \mathrm{~mL}+2 \times 30 \mathrm{~mL})$ were used. The crude material was purified by column chromatography (eluent: PE/EtOAc $=60 \%$ ) to afford the target product $\mathbf{2 c}$ as brown noncrystalline solid $(1.72 \mathrm{~g}, 59 \%)$.

${ }^{1} \mathrm{H}$ NMR (400 MHz, Chloroform- $d$ ) $\delta 8.46\left(\mathrm{ddd}, J=2.7 \mathrm{~Hz}, 1 \mathrm{H}, \mathrm{CH}_{\mathrm{Ar}}\right), 7.60(\mathrm{dd}, J=8.3,2.7$ $\left.\mathrm{Hz}, 1 \mathrm{H}, \mathrm{CH}_{\mathrm{Ar}}\right), 7.33\left(\mathrm{~d}, J=8.3 \mathrm{~Hz}, 1 \mathrm{H}, \mathrm{CH}_{\mathrm{Ar}}\right), 3.91\left(\mathrm{~s}, 2 \mathrm{H}, \mathrm{CH}_{2}\right), 3.50$ (s, 3H, $\left.\mathrm{SO}_{2} \mathrm{CH}_{2}\right), 2.65$ (s, $\left.3 \mathrm{H}, \mathrm{CH}_{3}\right)$.

${ }^{13} \mathrm{C}$ NMR (101 MHz, Chloroform- $\left.d\right) \delta 166.9(\mathrm{C}=\mathrm{O}), 161.7\left(\mathrm{C}_{\mathrm{Ar}}\right), 149.4\left(\mathrm{CH}_{\mathrm{Ar}}\right), 137.8\left(\mathrm{CH}_{\mathrm{Ar}}\right)$, $128.9\left(\mathrm{C}_{\mathrm{Ar}}\right), 124.6\left(\mathrm{CH}_{\mathrm{Ar}}\right), 43.2\left(\mathrm{CH}_{2}\right), 42.3\left(\mathrm{SO}_{2} \mathrm{CH}_{3}\right), 24.5\left(\mathrm{CH}_{3}\right)$.

IR (neat), $v\left(\mathrm{~cm}^{-1}\right)$ : 3013, 1718, 1621, 1561, 1487, 1317, 1147, 966, 894, 766.

HRMS (ESI) m/z: $[\mathrm{M}+\mathrm{H}]^{+}$Calcd for $\mathrm{C}_{9} \mathrm{H}_{12} \mathrm{ClN}_{2} \mathrm{O}_{3} \mathrm{~S}$ 263.0252; Found 263.0265.

$\mathrm{R}_{\mathrm{f}}=0.33(\mathrm{PE} / \mathrm{EtOAc}=60 \%)$. 
<smiles>CS(=O)(=O)N(C(=O)CCl)c1ccc(C(F)(F)F)nc1</smiles>

Following the general procedure $\mathrm{B}$, the reaction was carried out with sulfonamide $\mathbf{1 d}(2.72 \mathrm{~g}$, $11.3 \mathrm{mmol}, 1$ eq.) in DCM (23 mL), DIPEA (3.00 mL, $17.0 \mathrm{mmol}, 1.5$ eq.) and chloroacetyl chloride (1.80 mL, $22.6 \mathrm{mmol}, 2$ eq.) during $2 \mathrm{~h}$. Then it was diluted with DCM (30 mL), and poured into sat. $\mathrm{NH}_{4} \mathrm{Cl}$ solution $(50 \mathrm{~mL})$. The aqueous layer was extracted with $\mathrm{DCM}(30 \mathrm{~mL})$ and EtOAc $(30 \mathrm{~mL})$, and combined organic layers were washed with brine, dried over $\mathrm{MgSO}_{4}$ and concentrated under reduced pressure. The crude material was recrystallized from EtOAc in freezer to afford the target product $\mathbf{2 d}$ as white crystals $(2.63 \mathrm{~g}, 73 \%)$.

${ }^{1} \mathrm{H}$ NMR (400 MHz, DMSO- $\left.d_{6}\right) \delta 8.94\left(\mathrm{~d}, J=2.4 \mathrm{~Hz}, 1 \mathrm{H}, \mathrm{CH}_{\mathrm{Ar}}\right), 8.36(\mathrm{dd}, J=8.4,2.4 \mathrm{~Hz}$, $\left.1 \mathrm{H}, \mathrm{CH}_{\mathrm{Ar}}\right), 8.13\left(\mathrm{dd}, J=8.4 \mathrm{~Hz}, 1 \mathrm{H}, \mathrm{CH}_{\mathrm{Ar}}\right), 4.34\left(\mathrm{~s}, 2 \mathrm{H}, \mathrm{CH}_{2}\right), 3.68\left(\mathrm{~s}, 3 \mathrm{H}, \mathrm{SO}_{2} \mathrm{CH}_{3}\right)$.

${ }^{13} \mathrm{C}$ NMR (101 MHz, DMSO-d $\left.d_{6}\right) \delta 166.2(\mathrm{C}=\mathrm{O}), 151.6\left(\mathrm{CH}_{\mathrm{Ar}}\right), 147.1\left(\mathrm{C}-\mathrm{F},{ }^{2} J_{C-F}=34.4 \mathrm{~Hz}\right.$, $\left.\mathrm{C}_{\mathrm{Ar}}\right), 140.6\left(\mathrm{CH}_{\mathrm{Ar}}\right), 134.3\left(\mathrm{C}_{\mathrm{Ar}}\right), 121.9\left(\mathrm{C}-\mathrm{F},{ }^{3} J_{C-F}=2.8 \mathrm{~Hz}, \mathrm{CH}_{\mathrm{Ar}}\right), 121.3\left(\mathrm{C}-\mathrm{F},{ }^{1} J_{C-F}=274.4\right.$ $\left.\mathrm{Hz}, \mathrm{CF}_{3}\right), 44.8\left(\mathrm{CH}_{2}\right), 41.9\left(\mathrm{SO}_{2} \mathrm{CH}_{3}\right)$.

IR (neat), $v\left(\mathrm{~cm}^{-1}\right): 2939,1729,1333,1238,1139,1082,1030,968,895,778,760,705$.

HRMS (ESI) m/z: [M + H] ${ }^{+}$Calcd for $\mathrm{C}_{9} \mathrm{H}_{9} \mathrm{ClF}_{3} \mathrm{~N}_{2} \mathrm{O}_{3} \mathrm{~S}$ 316.9969; Found 316.9982.

$\mathrm{R}_{\mathrm{f}}=0.65(\mathrm{PE} / \mathrm{EtOAc}=50 \%)$.

m.p. $=187-189^{\circ} \mathrm{C}($ crystallized from EtOAc $)$. 
<smiles>CC(Cl)C(=O)N(c1ccc(Cl)nc1)S(C)(=O)=O</smiles>

Following the general procedure $\mathrm{B}$, the reaction was carried out with sulfonamide $1 \mathrm{e}(2.50 \mathrm{~g}$, $12.1 \mathrm{mmol}, 1$ eq.) in DCM (24 mL), DIPEA (3.20 mL, $18.2 \mathrm{mmol}, 1.5$ eq.) and chloropropionyl chloride (2.40 mL, $24.2 \mathrm{mmol}, 2$ eq.) during 2 h. For work-up, DCM (2 x $30 \mathrm{~mL}$ ) and sat. $\mathrm{NH}_{4} \mathrm{Cl}$ solution $(50 \mathrm{~mL})$ were used. The crude material was recrystallized from EtOAc in freezer to afford the target product $\mathbf{2 e}$ as white crystals $(2.06 \mathrm{~g}, 57 \%)$.

${ }^{1} \mathrm{H}$ NMR (400 MHz, DMSO- $\left.d_{6}\right) \delta 8.65\left(\mathrm{dd}, J=2.8,0.7 \mathrm{~Hz}, 1 \mathrm{H}, \mathrm{CH}_{\mathrm{Ar}}\right), 8.14(\mathrm{dd}, J=8.5,2.8$ $\left.\mathrm{Hz}, 1 \mathrm{H}, \mathrm{CH}_{\mathrm{Ar}}\right), 7.74\left(\mathrm{dd}, J=8.5,0.7 \mathrm{~Hz}, 1 \mathrm{H}, \mathrm{CH}_{\mathrm{Ar}}\right), 4.36(\mathrm{q}, J=6.4 \mathrm{~Hz}, 1 \mathrm{H}, \mathrm{CH}), 3.65$ (s, 3H, $\left.\mathrm{SO}_{2} \mathrm{CH}_{3}\right), 1.52\left(\mathrm{~d}, J=6.4 \mathrm{~Hz}, 3 \mathrm{H}, \mathrm{CH}_{3}\right)$.

${ }^{13} \mathrm{C}$ NMR (101 MHz, DMSO- $\left.d_{6}\right) \delta 168.7(\mathrm{C}=\mathrm{O}), 151.4\left(\mathrm{C}_{\mathrm{Ar}}\right), 151.4\left(\mathrm{CH}_{\mathrm{Ar}}\right), 141.8\left(\mathrm{CH}_{\mathrm{Ar}}\right)$, $131.0\left(\mathrm{C}_{\mathrm{Ar}}\right), 125.1\left(\mathrm{CH}_{\mathrm{Ar}}\right), 51.8(\mathrm{CH}), 41.6\left(\mathrm{SO}_{2} \mathrm{CH}_{3}\right), 20.1\left(\mathrm{CH}_{3}\right)$.

IR (neat), $v\left(\mathrm{~cm}^{-1}\right): 3069,1712,1562,1456,1343,1273,1154,1115,963,861,775,748$.

HRMS (ESI) m/z: [M + H] ${ }^{+}$Calcd for $\mathrm{C}_{9} \mathrm{H}_{11} \mathrm{Cl}_{2} \mathrm{~N}_{2} \mathrm{O}_{3} \mathrm{~S} 296.9862$; Found 296.9876.

$\mathrm{R}_{\mathrm{f}}=0.60(\mathrm{PE} / \mathrm{EtOAc}=50 \%)$.

m.p. $=134-137^{\circ} \mathrm{C}($ crystallized from EtOAc $)$. 
<smiles>CS(=O)(=O)N(C(=O)CCl)c1ccnc(Cl)c1</smiles>

Following the general procedure $\mathrm{B}$, the reaction was carried out with sulfonamide $\mathbf{1 f}(2.50 \mathrm{~g}$, $12.1 \mathrm{mmol}, 1$ eq.) in DCM (24 mL), DIPEA (3.20 mL, $18.2 \mathrm{mmol}, 1.5$ eq.) and chloroacetyl chloride (1.90 mL, $24.2 \mathrm{mmol}, 2$ eq.) during 2 h. For work-up, DCM (50 mL + $25 \mathrm{~mL})$ and sat. $\mathrm{NH}_{4} \mathrm{Cl}$ solution $(2 \times 50 \mathrm{~mL})$ were used. The crude material was recrystallized from EtOAc in freezer to afford the target product $\mathbf{2 f}$ as white crystals $(1.89 \mathrm{~g}, 55 \%)$.

${ }^{1} \mathrm{H}$ NMR (400 MHz, Acetone- $\left.d_{6}\right) \delta 8.61\left(\mathrm{dd}, J=5.2,0.6 \mathrm{~Hz}, 1 \mathrm{H}, \mathrm{CH}_{\mathrm{Ar}}\right), 7.78(\mathrm{dd}, J=1.8,0.6$ $\left.\mathrm{Hz}, 1 \mathrm{H}, \mathrm{CH}_{\mathrm{Ar}}\right), 7.66\left(\mathrm{dd}, J=5.2,1.8 \mathrm{~Hz}, 1 \mathrm{H}, \mathrm{CH}_{\mathrm{Ar}}\right), 4.34\left(\mathrm{~s}, 2 \mathrm{H}, \mathrm{CH}_{2}\right), 3.62\left(\mathrm{~s}, 3 \mathrm{H}, \mathrm{SO}_{2} \mathrm{CH}_{3}\right)$. ${ }^{13} \mathrm{C}$ NMR $\left(101 \mathrm{MHz}\right.$, Acetone- $\left.d_{6}\right) \delta 166.4(\mathrm{C}=\mathrm{O}), 153.0\left(\mathrm{C}_{\mathrm{Ar}}\right), 152.2\left(\mathrm{CH}_{\mathrm{Ar}}\right), 145.9\left(\mathrm{C}_{\mathrm{Ar}}\right), 126.9$ $\left(\mathrm{CH}_{\mathrm{Ar}}\right), 125.6\left(\mathrm{CH}_{\mathrm{Ar}}\right), 44.9\left(\mathrm{CH}_{2}\right), 42.5\left(\mathrm{SO}_{2} \mathrm{CH}_{3}\right)$.

IR (neat), $v\left(\mathrm{~cm}^{-1}\right)$ : 1725, 1697, 1578, 1461, 1356, 1251, 1154, 1120, 1084, 965, 911, 777, 747. HRMS (ESI) m/z: $[\mathrm{M}+\mathrm{H}]^{+}$Calcd for $\mathrm{C}_{8} \mathrm{H}_{9} \mathrm{Cl}_{2} \mathrm{~N}_{2} \mathrm{O}_{3} \mathrm{~S}$ 282.9705; Found 282.9707. $\mathrm{R}_{\mathrm{f}}=0.48(\mathrm{PE} / \mathrm{EtOAc}=40 \%)$. m.p. $=122-125^{\circ} \mathrm{C}($ crystallized from EtOAc). 
<smiles>CS(=O)(=O)N(C(=O)CCl)c1cccc(Cl)n1</smiles>

Following the general procedure B, the reaction was carried out with sulfonamide $1 \mathrm{~g}$ (3.20 g, $15.5 \mathrm{mmol}, 1$ eq.) in DCM (31 mL), DIPEA (4.10 mL, $23.2 \mathrm{mmol}, 1.5$ eq.) and chloroacetyl chloride (2.50 mL, $31.5 \mathrm{mmol}, 2$ eq.) during $2 \mathrm{~h}$. For work-up, DCM (50 mL $+25 \mathrm{~mL})$ and sat. $\mathrm{NH}_{4} \mathrm{Cl}$ solution $(2 \times 50 \mathrm{~mL}$ ) were used. The crude material was recrystallized from EtOAc in freezer to afford the target product $\mathbf{2} \mathbf{g}$ as white crystals $(3.89 \mathrm{~g}, 77 \%)$.

${ }^{1} \mathrm{H}$ NMR (400 MHz, Acetone- $\left.d_{6}\right) \delta 8.13\left(\mathrm{dd}, J=7.7 \mathrm{~Hz}, 1 \mathrm{H}, \mathrm{CH}_{\mathrm{Ar}}\right), 7.71(\mathrm{dd}, J=6.0,0.7 \mathrm{~Hz}$, $\left.1 \mathrm{H}, \mathrm{CH}_{\mathrm{Ar}}\right), 7.69\left(\mathrm{dd}, J=6.3,0.7 \mathrm{~Hz}, 1 \mathrm{H}, \mathrm{CH}_{\mathrm{Ar}}\right), 4.31\left(\mathrm{~s}, 2 \mathrm{H}, \mathrm{CH}_{2}\right), 3.57\left(\mathrm{~s}, 3 \mathrm{H}, \mathrm{SO}_{2} \mathrm{CH}_{3}\right)$.

${ }^{13} \mathrm{C}$ NMR $\left(101 \mathrm{MHz}\right.$, Acetone- $\left.d_{6}\right) \delta 167.2(\mathrm{C}=\mathrm{O}), 151.2\left(\mathrm{C}_{\mathrm{Ar}}\right), 149.2\left(\mathrm{C}_{\mathrm{Ar}}\right), 143.1\left(\mathrm{CH}_{\mathrm{Ar}}\right), 127.1$ $\left(\mathrm{CH}_{\mathrm{Ar}}\right), 126.0\left(\mathrm{CH}_{\mathrm{Ar}}\right), 44.7\left(\mathrm{CH}_{2}\right), 42.3\left(\mathrm{SO}_{2} \mathrm{CH}_{3}\right)$.

IR (neat), $v\left(\mathrm{~cm}^{-1}\right)$ : 2939, 1728, 1579, 1569, 1429, 1359, 1319, 1158, 1026, 967, 779, 747. HRMS (ESI) m/z: $[\mathrm{M}+\mathrm{H}]^{+}$Calcd for $\mathrm{C}_{8} \mathrm{H}_{9} \mathrm{Cl}_{2} \mathrm{~N}_{2} \mathrm{O}_{3} \mathrm{~S}$ 282.9705; Found 282.9708.

$\mathrm{R}_{\mathrm{f}}=0.58(\mathrm{PE} / \mathrm{EtOAc}=40 \%)$.

m.p. $=151-153^{\circ} \mathrm{C}($ crystallized from EtOAc $)$. 


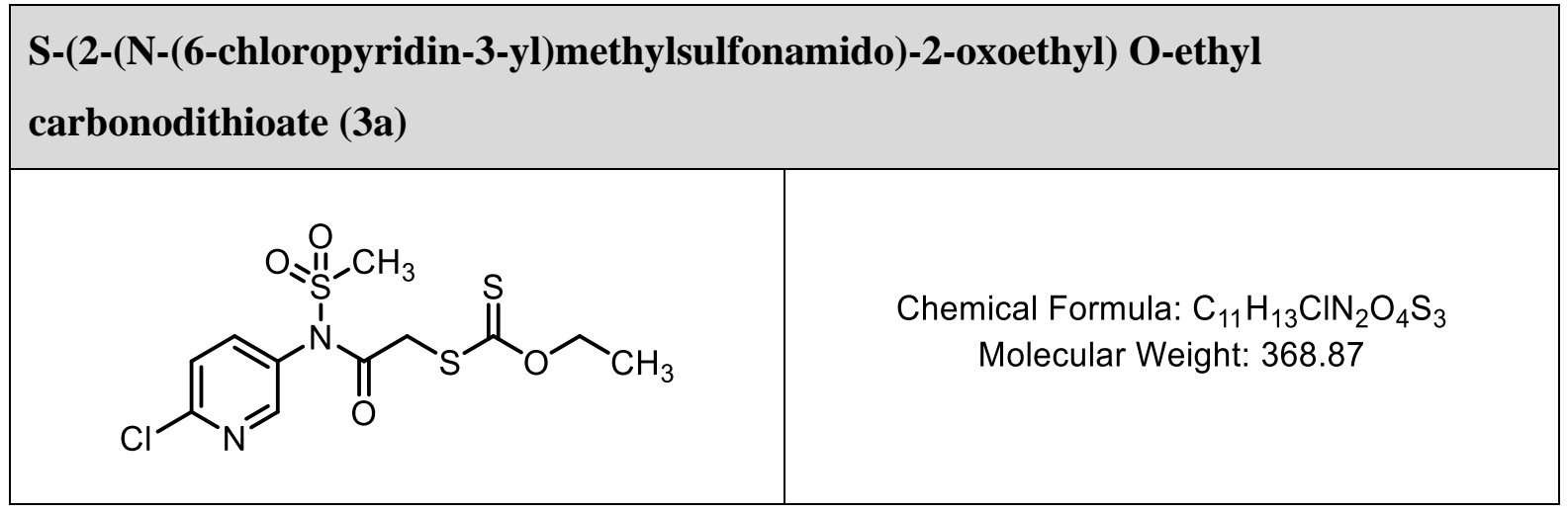

Following the general procedure for the formation of xanthate (procedure $\mathrm{C}$ ), the reaction was carried out with chloride 2a (12.0 g, $42.4 \mathrm{mmol}, 1$ eq.) in acetone (140 mL) and potassium ethyl xanthogenate (7.14 g, 44.5 mmol, 1.05 eq.) during 3 h. For work-up, EtOAc $200 \mathrm{~mL}$ ) and brine $(2 \times 150 \mathrm{~mL})$ were used. The crude material was purified by flash column chromatography (eluent: PE/EtOAc $=40 \%$ ) to afford the target product 3a as a brown oil, that solidified upon time $(14.2 \mathrm{~g}, 91 \%)$.

${ }^{1} \mathrm{H}$ NMR (400 MHz, Chloroform-d) $\delta 8.46\left(\mathrm{dd}, \mathrm{J}=2.8,0.7 \mathrm{~Hz}, 1 \mathrm{H}, \mathrm{CH}_{\mathrm{Ar}}\right), 7.78(\mathrm{dd}, \mathrm{J}=8.4$, $\left.2.8 \mathrm{~Hz}, 1 \mathrm{H}, \mathrm{CH}_{\mathrm{Ar}}\right), 7.51\left(\mathrm{dd}, \mathrm{J}=8.4,0.7 \mathrm{~Hz}, 1 \mathrm{H}, \mathrm{CH}_{\mathrm{Ar}}\right.$ ), $4.61\left(\mathrm{q}, \mathrm{J}=7.1 \mathrm{~Hz}, 2 \mathrm{H}, \mathrm{CH}_{2}\right), 3.80$ (s, $\left.2 \mathrm{H}, \mathrm{CH}_{2}\right), 3.49\left(\mathrm{~s}, 3 \mathrm{H}, \mathrm{SO}_{2} \mathrm{CH}_{3}\right), 1.41\left(\mathrm{t}, \mathrm{J}=7.1 \mathrm{~Hz}, 3 \mathrm{H}, \mathrm{CH}_{3}\right)$.

${ }^{13} \mathrm{C}$ NMR (101 MHz, Chloroform- $\left.d\right) \delta 213.4(\mathrm{C}=\mathrm{S}), 167.2(\mathrm{C}=\mathrm{O}), 153.4\left(\mathrm{C}_{\mathrm{Ar}}\right), 150.4\left(\mathrm{CH}_{\mathrm{Ar}}\right)$, 140.4 $\left(\mathrm{CH}_{\mathrm{Ar}}\right), 131.1\left(\mathrm{C}_{\mathrm{Ar}}\right), 125.7\left(\mathrm{CH}_{\mathrm{Ar}}\right), 71.7\left(\mathrm{CH}_{2}\right), 42.5\left(\mathrm{SO}_{2} \mathrm{CH}_{3}\right), 41.4\left(\mathrm{CH}_{2}\right), 13.8\left(\mathrm{CH}_{3}\right)$.

IR (neat): 2985, 2931, 1713, 1562, 1454, 1355, 1288, 1149, 1108, 1045, 961, 871, 771, 746.

HRMS (ESI) m/z: [M + H] ${ }^{+}$Calcd for $\mathrm{C}_{11} \mathrm{H}_{14} \mathrm{ClN}_{2} \mathrm{O}_{4} \mathrm{~S}_{3} 368.9799$; Found 368.9801 .

$\mathrm{R}_{\mathrm{f}}=0.74(\mathrm{PE} / \mathrm{EtOAc}=40 \%)$.

m.p. $=103-105^{\circ} \mathrm{C}$. 


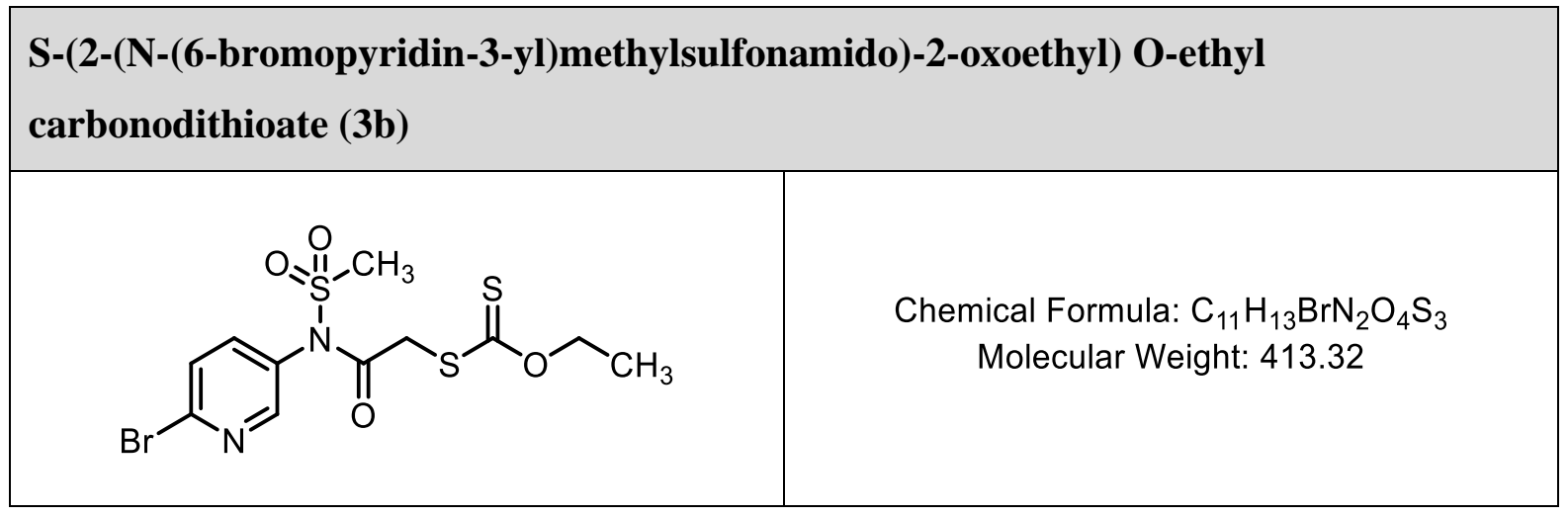

Following the general procedure for the formation of xanthate (procedure $\mathrm{C}$ ), the reaction was carried out with chloride $\mathbf{2 b}(2.54 \mathrm{~g}, 25.9 \mathrm{mmol}, 1$ eq.) in acetone $(26 \mathrm{~mL})$ and potassium ethyl xanthogenate (1.31 g, $8.14 \mathrm{mmol}, 1.05$ eq.) during 4 h. For work-up, EtOAc $(50 \mathrm{~mL}+25 \mathrm{~mL})$ and water $(50 \mathrm{~mL})$ were used. The crude material was purified by flash column chromatography (eluent: $\mathrm{PE} / \mathrm{EtOAc}=40 \%)$ to afford the target product as a white solid $(2.61$ g, $82 \%)$.

${ }^{1} \mathrm{H}$ NMR (400 MHz, Chloroform- $d$ ) $\delta 8.44\left(\mathrm{dd}, J=2.0,1.5 \mathrm{~Hz}, 1 \mathrm{H}, \mathrm{CH}_{\mathrm{Ar}}\right), 7.67\left(\mathrm{~m}, 1 \mathrm{H}, \mathrm{CH}_{\mathrm{Ar}}\right)$, $7.66\left(\mathrm{~m}, 1 \mathrm{H}, \mathrm{CH}_{\mathrm{Ar}}\right), 4.61\left(\mathrm{q}, J=7.1 \mathrm{~Hz}, 2 \mathrm{H}, \mathrm{CH}_{2}\right), 3.79\left(\mathrm{~s}, 2 \mathrm{H}, \mathrm{CH}_{2}\right), 3.49\left(\mathrm{~s}, 3 \mathrm{H}, \mathrm{SO}_{2} \mathrm{CH}_{3}\right)$, $1.41\left(\mathrm{t}, J=7.1 \mathrm{~Hz}, 3 \mathrm{H}, \mathrm{CH}_{3}\right)$.

${ }^{13} \mathrm{C}$ NMR $(101 \mathrm{MHz}$, Chloroform- $d) \delta 213.3(\mathrm{C}=\mathrm{S}), 167.1(\mathrm{C}=\mathrm{O}), 150.8\left(\mathrm{CH}_{\mathrm{Ar}}\right), 144.1\left(\mathrm{C}_{\mathrm{Ar}}\right)$, $140.1\left(\mathrm{CH}_{\mathrm{Ar}}\right), 131.5\left(\mathrm{C}_{\mathrm{Ar}}\right), 129.6\left(\mathrm{CH}_{\mathrm{Ar}}\right), 71.7\left(\mathrm{CH}_{2}\right), 42.5\left(\mathrm{SO}_{2} \mathrm{CH}_{3}\right), 41.4\left(\mathrm{CH}_{2}\right), 13.8\left(\mathrm{CH}_{3}\right)$.

IR (neat), $v\left(\mathrm{~cm}^{-1}\right): 3048,1698,1555,1449,1345,1307,1217,1150,1090,1045,962,891$, 768.

HRMS (ESI) m/z: $[\mathrm{M}+\mathrm{H}]^{+}$Calcd for $\mathrm{C}_{11} \mathrm{H}_{14} \mathrm{BrN}_{2} \mathrm{O}_{4} \mathrm{~S}_{3} 412.9294$; Found 412.9310 .

$\mathrm{R}_{\mathrm{f}}=0.63(\mathrm{PE} / \mathrm{EtOAc}=40 \%)$.

m.p. $=118-122^{\circ} \mathrm{C}$. 


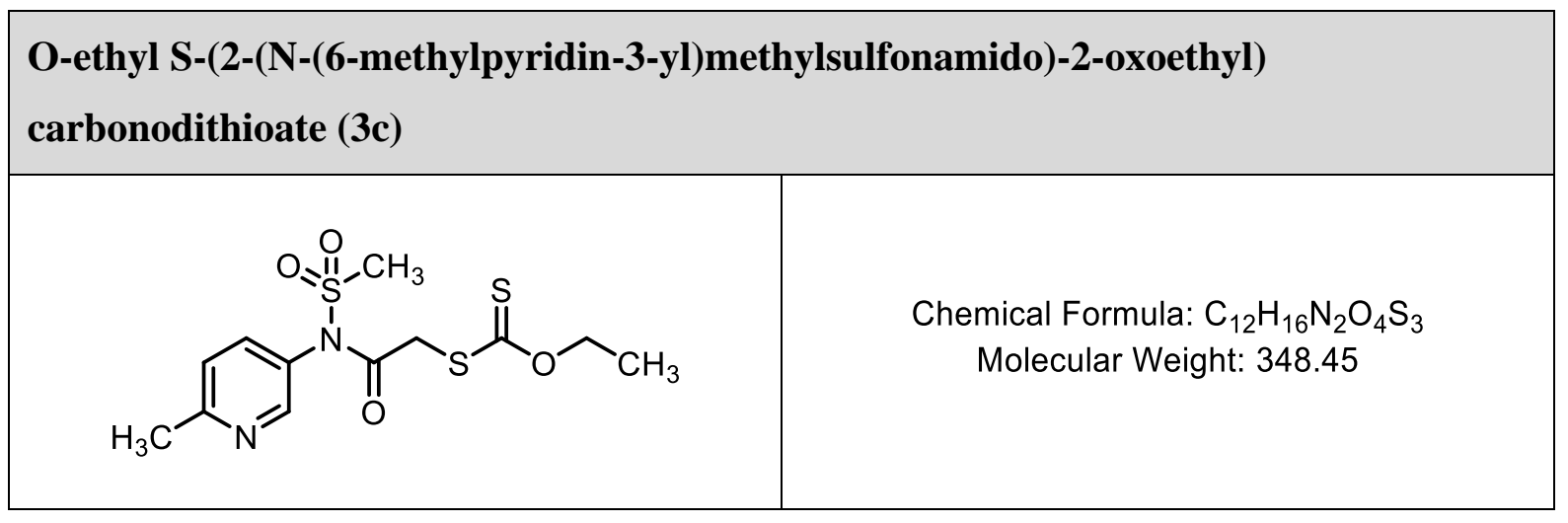

Following the general procedure for the formation of xanthate (procedure C), the reaction was carried out with chloride $2 \mathrm{c}$ (1.65 g, $6.28 \mathrm{mmol}, 1$ eq.) in acetone $(21 \mathrm{~mL})$ and potassium ethyl xanthogenate (1.06 g, $6.59 \mathrm{mmol}, 1.05$ eq.) during 4 h. For work-up, EtOAc $(50 \mathrm{~mL}+25 \mathrm{~mL})$ and water $(50 \mathrm{~mL})$ were used. The crude material was purified by flash column chromatography (eluent: $\mathrm{PE} / \mathrm{EtOAc}=50 / 50 \rightarrow 40 / 60)$ to afford the target product $\mathbf{3 c}$ as a white solid $(1.76 \mathrm{~g}$, $80 \%)$.

${ }^{1} \mathrm{H}$ NMR (400 MHz, Chloroform- $d$ ) $\delta 8.53\left(\mathrm{dd}, J=2.7,0.8 \mathrm{~Hz}, 1 \mathrm{H}, \mathrm{CH}_{\mathrm{Ar}}\right), 7.67(\mathrm{dd}, J=8.3$, $\left.2.7 \mathrm{~Hz}, 1 \mathrm{H}, \mathrm{CH}_{\mathrm{Ar}}\right), 7.32\left(\mathrm{dd}, J=8.3,0.8 \mathrm{~Hz}, 1 \mathrm{H}, \mathrm{CH}_{\mathrm{Ar}}\right.$ ), 4.59 (q, $\left.J=7.1 \mathrm{~Hz}, 2 \mathrm{H}, \mathrm{CH}_{2}\right), 3.75$ (s, $\left.2 \mathrm{H}, \mathrm{CH}_{2}\right), 3.47$ (s, 3H, $\left.\mathrm{SO}_{2} \mathrm{CH}_{3}\right), 2.62\left(\mathrm{~s}, 3 \mathrm{H}, \mathrm{CH}_{3}\right), 1.39$ (t, J = 7.1 Hz, 3H, $\left.\mathrm{CH}_{3}\right)$.

${ }^{13} \mathrm{C}$ NMR $(101 \mathrm{MHz}$, Chloroform- $d) \delta 213.2(\mathrm{C}=\mathrm{S}), 167.5(\mathrm{C}=\mathrm{O}), 161.2\left(\mathrm{C}_{\mathrm{Ar}}\right), 149.6\left(\mathrm{CH}_{\mathrm{Ar}}\right)$, $137.8\left(\mathrm{CH}_{\mathrm{Ar}}\right), 129.4\left(\mathrm{C}_{\mathrm{Ar}}\right), 124.5\left(\mathrm{CH}_{\mathrm{Ar}}\right), 71.4\left(\mathrm{CH}_{2}\right), 42.3\left(\mathrm{SO}_{2} \mathrm{CH}_{3}\right), 41.4\left(\mathrm{CH}_{2}\right), 24.5\left(\mathrm{CH}_{3}\right)$, $13.8\left(\mathrm{CH}_{3}\right)$.

IR (neat), $v\left(\mathrm{~cm}^{-1}\right): 2988,1701,1484,1343,1306,1215,1150,1109,1045,964,893,764$.

HRMS (ESI) m/z: $[\mathrm{M}+\mathrm{H}]^{+}$Calcd for $\mathrm{C}_{12} \mathrm{H}_{17} \mathrm{~N}_{2} \mathrm{O}_{4} \mathrm{~S}_{3}$ 349.0345; Found 349.0358.

$\mathrm{R}_{\mathrm{f}}=0.19(\mathrm{PE} / \mathrm{EtOAc}=40 \%)$.

m.p. $=117-118^{\circ} \mathrm{C}$. 


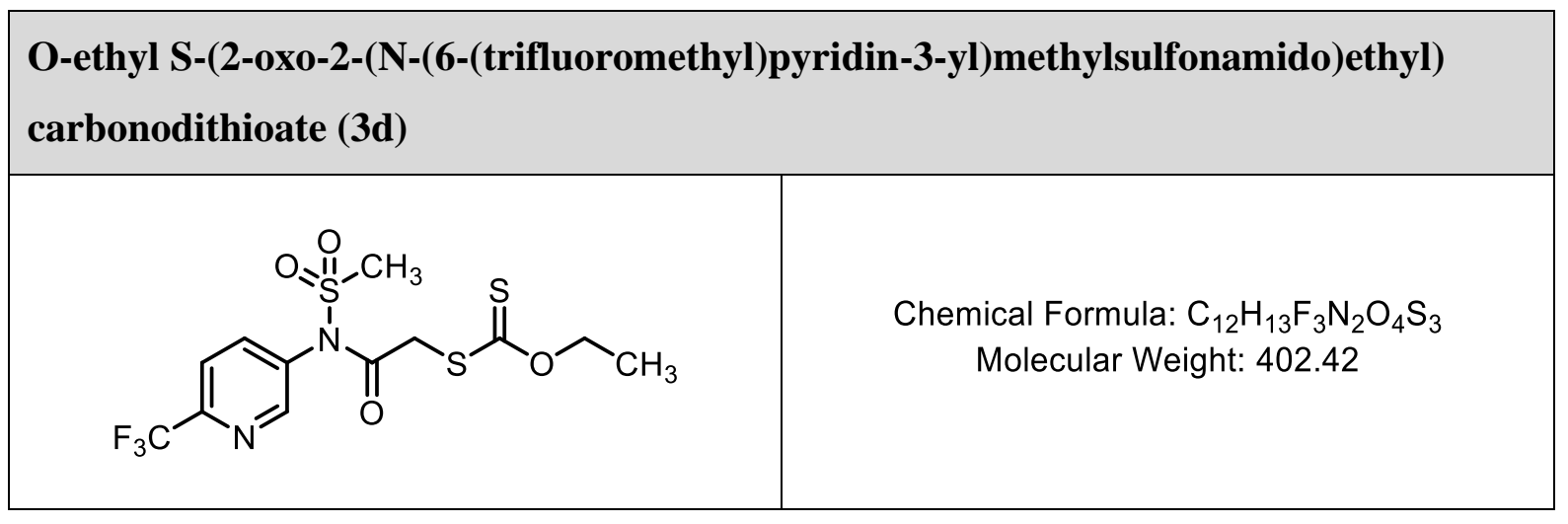

Following the general procedure for the formation of xanthate (procedure C), the reaction was carried out with chloride $2 \mathbf{d}$ ( $2.49 \mathrm{~g}, 7.85 \mathrm{mmol}, 1$ eq.) in acetone (26 mL) and potassium ethyl xanthogenate (1.32 g, $8.24 \mathrm{mmol}, 1.05$ eq.) during 2.5 h. For work-up, EtOAc $(50+30 \mathrm{~mL})$ and brine $(50 \mathrm{~mL})$ were used. The crude material was purified by flash column chromatography (eluent: PE/EtOAc $=80 / 20 \rightarrow 60 / 40)$ to afford the target product 3d as a yellow oil $(2.54 \mathrm{~g}$, $80 \%$ ), that solidified into a white solid.

${ }^{1} \mathrm{H}$ NMR (400 MHz, Chloroform- $d$ ) $\delta 8.79\left(\mathrm{dd}, J=2.5,0.8 \mathrm{~Hz}, 1 \mathrm{H}, \mathrm{CH}_{\mathrm{Ar}}\right), 8.02(\mathrm{dd}, J=8.4$, $\left.2.5 \mathrm{~Hz}, 1 \mathrm{H}, \mathrm{CH}_{\mathrm{Ar}}\right), 7.87\left(\mathrm{dd}, J=8.4,0.8 \mathrm{~Hz}, 1 \mathrm{H}, \mathrm{CH}_{\mathrm{Ar}}\right.$ ), $4.62\left(\mathrm{q}, J=7.1 \mathrm{~Hz}, 2 \mathrm{H}, \mathrm{CH}_{2}\right), 3.82$ (s, $\left.2 \mathrm{H}, \mathrm{CH}_{2}\right), 3.53$ (s, $\left.3 \mathrm{H}, \mathrm{SO}_{2} \mathrm{CH}_{3}\right), 1.42\left(\mathrm{t}, J=7.1 \mathrm{~Hz}, 3 \mathrm{H}, \mathrm{CH}_{3}\right)$.

${ }^{13} \mathrm{C}$ NMR (101 MHz, Chloroform- $\left.d\right) \delta 213.4(\mathrm{C}=\mathrm{S}), 167.0(\mathrm{C}=\mathrm{O}), 150.9\left(\mathrm{CH}_{\mathrm{Ar}}\right), 149.6(\mathrm{C}-\mathrm{F}$, $\left.{ }^{2} J_{C-F}=35.7 \mathrm{~Hz}, \mathrm{C}_{\mathrm{Ar}}\right), 139.6\left(\mathrm{CH}_{\mathrm{Ar}}\right), 134.5\left(\mathrm{C}_{\mathrm{Ar}}\right), 121.8\left(\mathrm{C}-\mathrm{F},{ }^{3} J_{C-F}=2.8 \mathrm{~Hz}, \mathrm{CH}_{\mathrm{Ar}}\right), 121.0(\mathrm{C}-$ $\left.\mathrm{F},{ }^{1} J_{C-F}=274.6 \mathrm{~Hz}, \mathrm{CF}_{3}\right), 71.8\left(\mathrm{CH}_{2}\right), 42.5\left(\mathrm{SO}_{2} \mathrm{CH}_{3}\right), 41.6\left(\mathrm{CH}_{2}\right), 13.8\left(\mathrm{CH}_{3}\right)$.

IR (neat), $v\left(\mathrm{~cm}^{-1}\right): 2898,1714,1360,1338,1242,1158,1130,1086,1040,964,904,774,755$. HRMS (ESI) m/z: $[\mathrm{M}+\mathrm{H}]^{+}$Calcd for $\mathrm{C}_{12} \mathrm{H}_{14} \mathrm{~F}_{3} \mathrm{~N}_{2} \mathrm{O}_{4} \mathrm{~S}_{3}$ 403.0062; Found 403.0080.

$\mathrm{R}_{\mathrm{f}}=0.71(\mathrm{PE} / \mathrm{EtOAc}=40 \%)$.

m.p. $=107-109^{\circ} \mathrm{C}$. 


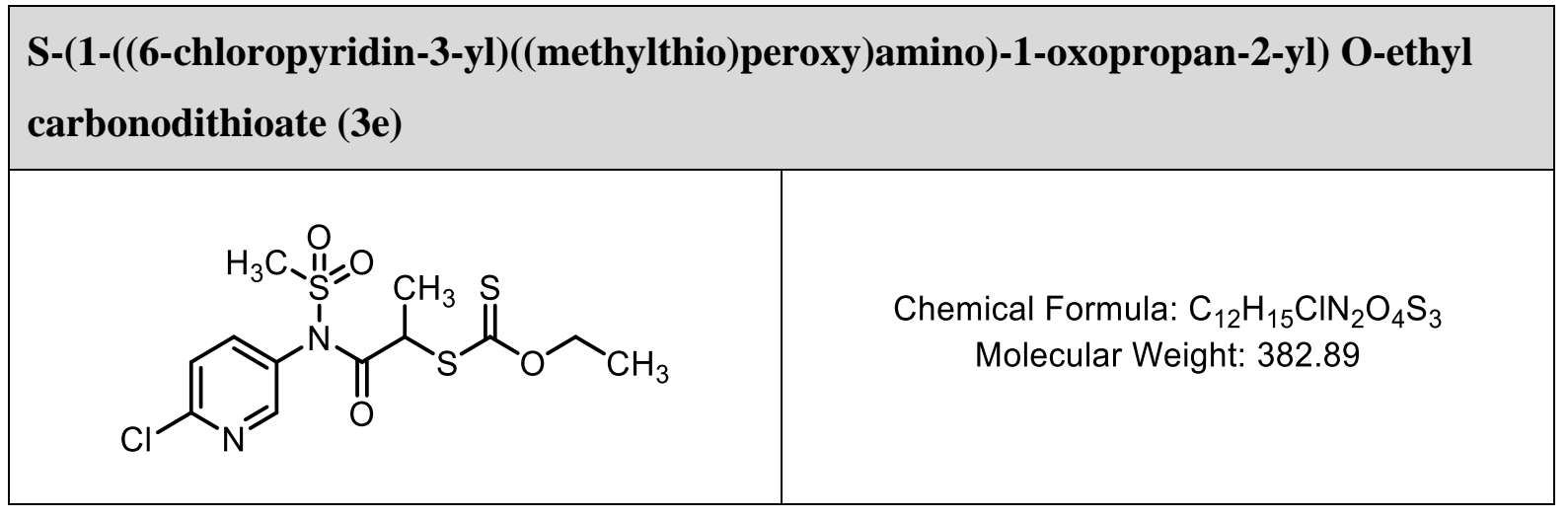

Following the general procedure for the formation of xanthate (procedure $\mathrm{C}$ ), the reaction was carried out with chloride $2 \mathrm{e}$ (1.92 g, $6.45 \mathrm{mmol}, 1$ eq.) in acetone $(22 \mathrm{~mL})$ and potassium ethyl xanthogenate (1.09 g, $6.77 \mathrm{mmol}, 1.05$ eq.) during 2.5 h. For work-up, EtOAc $(100 \mathrm{~mL}+50$ $\mathrm{mL})$ and brine $(100 \mathrm{~mL})$ were used. The crude material was recrystallized from PE/EtOAc in freezer to afford the target product $\mathbf{3 e}$ as white crystals $(1.77 \mathrm{~g}, 72 \%)$.

${ }^{1} \mathrm{H}$ NMR (400 MHz, Chloroform- $d$ ) $\delta 8.45\left(\mathrm{dd}, J=2.8,0.7 \mathrm{~Hz}, 1 \mathrm{H}, \mathrm{CH}_{\mathrm{Ar}}\right), 7.77(\mathrm{dd}, J=8.4$, $\left.2.8 \mathrm{~Hz}, 1 \mathrm{H}, \mathrm{CH}_{\mathrm{Ar}}\right), 7.45\left(\mathrm{dd}, J=8.4,0.7 \mathrm{~Hz}, 1 \mathrm{H}, \mathrm{CH}_{\mathrm{Ar}}\right), 4.63-4.47\left(\mathrm{~m}, 2 \mathrm{H}, \mathrm{CH}_{2}\right), 4.21(\mathrm{q}, J$ $=7.1 \mathrm{~Hz}, 1 \mathrm{H}, \mathrm{CH}), 3.49\left(\mathrm{~s}, 3 \mathrm{H}, \mathrm{SO}_{2} \mathrm{CH}_{3}\right), 1.49\left(\mathrm{~d}, J=7.1 \mathrm{~Hz}, 3 \mathrm{H}, \mathrm{CH}_{3}\right), 1.38(\mathrm{t}, J=7.1 \mathrm{~Hz}$, $\left.3 \mathrm{H}, \mathrm{CH}_{3}\right)$.

${ }^{13} \mathrm{C}$ NMR (101 MHz, Chloroform- $\left.d\right) \delta 211.9(\mathrm{C}=\mathrm{S}), 171.3(\mathrm{C}=\mathrm{O}), 153.2\left(\mathrm{C}_{\mathrm{Ar}}\right), 150.7\left(\mathrm{CH}_{\mathrm{Ar}}\right)$, $140.7\left(\mathrm{CH}_{\mathrm{Ar}}\right), 130.7\left(\mathrm{C}_{\mathrm{Ar}}\right), 125.4\left(\mathrm{CH}_{\mathrm{Ar}}\right), 71.5\left(\mathrm{CH}_{2}\right), 48.4(\mathrm{CH}), 42.4\left(\mathrm{SO}_{2} \mathrm{CH}_{3}\right), 16.7\left(\mathrm{CH}_{3}\right)$, $13.8\left(\mathrm{CH}_{3}\right)$.

IR (neat), $v\left(\mathrm{~cm}^{-1}\right): 2987,1701,1565,1451,1339,1233,1211,1143,1026,954,864,768,750$. HRMS (ESI) m/z: $[\mathrm{M}+\mathrm{H}]^{+}$Calcd for $\mathrm{C}_{12} \mathrm{H}_{16} \mathrm{ClN}_{2} \mathrm{O}_{4} \mathrm{~S}_{3}$ 382.9955; Found 382.9973.

$\mathrm{R}_{\mathrm{f}}=0.70(\mathrm{PE} / \mathrm{EtOAc}=40 \%)$.

m.p. $=128-130^{\circ} \mathrm{C}($ crystallized from PE/EtOAc). 


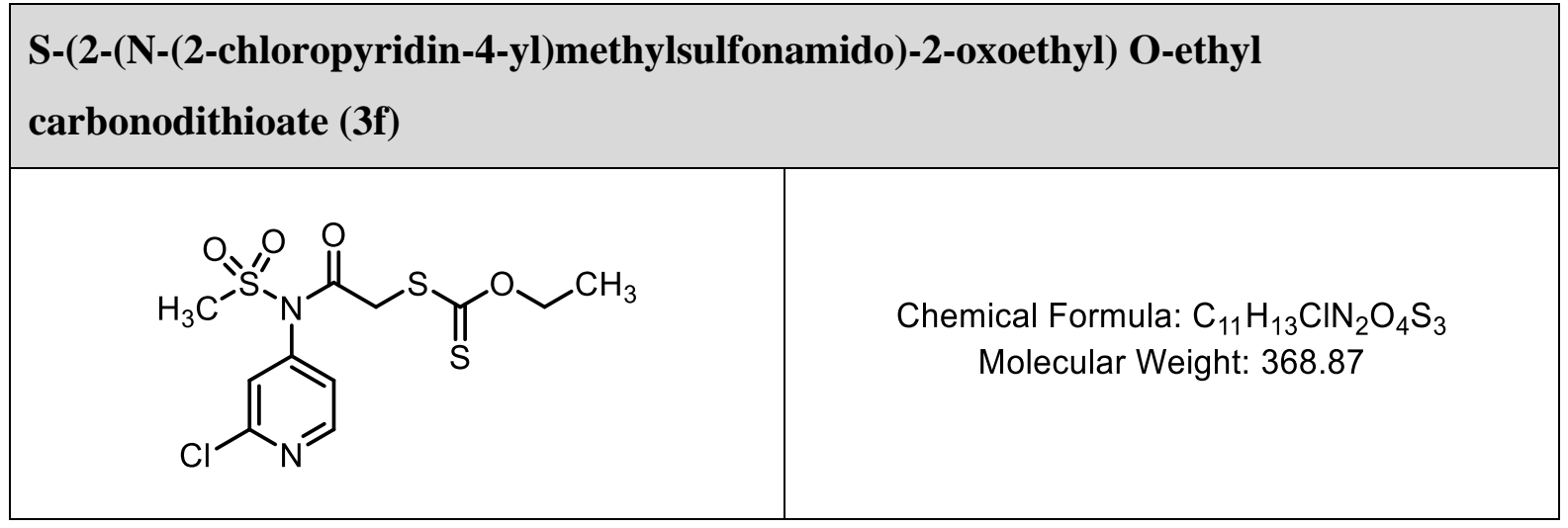

Following the general procedure for the formation of xanthate (procedure $\mathrm{C}$ ), the reaction was carried out with chloride $\mathbf{2 f}(1.75 \mathrm{~g}, 6.18 \mathrm{mmol}, 1$ eq. $)$ in acetone $(20 \mathrm{~mL})$ and potassium ethyl xanthogenate (1.04 g, 6.49 mmol, 1.05 eq.) during 2 h. For work-up, EtOAc (30 mL) and brine $(30 \mathrm{~mL})$ were used. The crude material was purified by column chromatography (eluent: $\mathrm{PE} / \mathrm{EtOAc}=80 / 20 \rightarrow 70 / 30)$ to afford the target product $\mathbf{3 f}$ as yellow oil $(1.63 \mathrm{~g}, 71 \%)$.

${ }^{1} \mathrm{H}$ NMR (400 MHz, Chloroform- $d$ ) $\delta 8.57$ (d, $J=5.2 \mathrm{~Hz}, 1 \mathrm{H}, \mathrm{CH}_{\mathrm{Ar}}$ ), 7.45 (d, $J=1.8 \mathrm{~Hz}, 1 \mathrm{H}$, $\left.\mathrm{CH}_{\mathrm{Ar}}\right), 7.36\left(\mathrm{dd}, J=5.2,1.8 \mathrm{~Hz}, 1 \mathrm{H}, \mathrm{CH}_{\mathrm{Ar}}\right), 4.61\left(\mathrm{q}, J=7.1 \mathrm{~Hz}, 2 \mathrm{H}, \mathrm{CH}_{2}\right), 3.81\left(\mathrm{~s}, 2 \mathrm{H}, \mathrm{CH}_{2}\right)$, $3.48\left(\mathrm{~s}, 3 \mathrm{H}, \mathrm{SO}_{2} \mathrm{CH}_{3}\right), 1.40\left(\mathrm{t}, J=7.1 \mathrm{~Hz}, 3 \mathrm{H}, \mathrm{CH}_{3}\right)$.

${ }^{13} \mathrm{C}$ NMR (101 MHz, Chloroform- $\left.d\right) \delta 213.3(\mathrm{C}=\mathrm{S}), 166.6(\mathrm{C}=\mathrm{O}), 153.2\left(\mathrm{C}_{\mathrm{Ar}}\right), 151.6\left(\mathrm{CH}_{\mathrm{Ar}}\right)$, $144.8\left(\mathrm{C}_{\mathrm{Ar}}\right), 125.7\left(\mathrm{CH}_{\mathrm{Ar}}\right), 123.8\left(\mathrm{CH}_{\mathrm{Ar}}\right), 71.7\left(\mathrm{CH}_{2}\right), 42.7\left(\mathrm{SO}_{2} \mathrm{CH}_{3}\right), 41.3\left(\mathrm{CH}_{2}\right), 13.8\left(\mathrm{CH}_{3}\right)$.

IR (neat), $v\left(\mathrm{~cm}^{-1}\right): 1716,1578,1461,1359,1231,1151,1113,1046,965,773$.

HRMS (ESI) m/z: [M + H] ${ }^{+}$Calcd for $\mathrm{C}_{11} \mathrm{H}_{14} \mathrm{ClN}_{2} \mathrm{O}_{4} \mathrm{~S}_{3} 368.9799$; Found 368.9800 .

$\mathrm{R}_{\mathrm{f}}=0.59(\mathrm{PE} / \mathrm{EtOAc}=40 \%)$. 


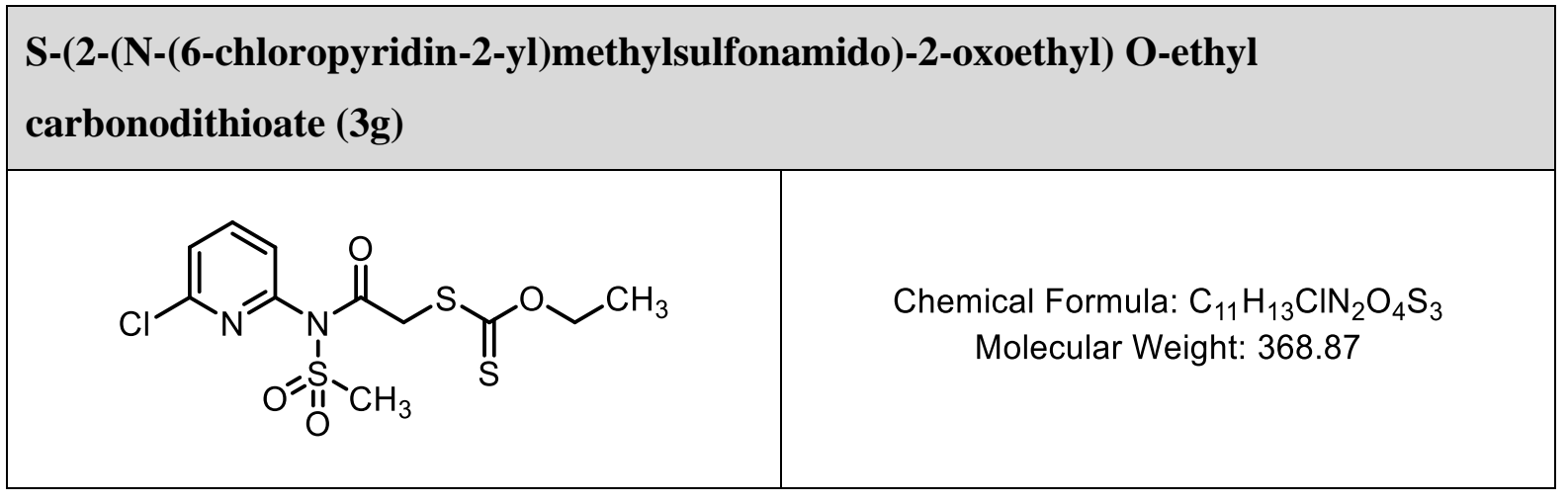

Following the general procedure for the formation of xanthate (procedure $\mathrm{C}$ ), the reaction was carried out with chloride $2 \mathrm{~g}$ ( $3.20 \mathrm{~g}, 11.3 \mathrm{mmol}, 1$ eq.) in acetone (38 $\mathrm{mL})$ and potassium ethyl xanthogenate (1.90 g, $11.9 \mathrm{mmol}, 1.05$ eq.) during $2 \mathrm{~h}$. For work-up, EtOAc (3 x $30 \mathrm{~mL})$ and $\mathrm{H}_{2} \mathrm{O}(2 \times 30 \mathrm{~mL})$ were used. The crude material was recrystallized from EtOAc in freezer to afford the target product $\mathbf{3 g}$ as white crystals $(2.63 \mathrm{~g}, 63 \%)$.

${ }^{1} \mathrm{H}$ NMR (400 MHz, Chloroform- $d$ ) $\delta 7.89\left(\mathrm{dd}, J=7.9,7.7 \mathrm{~Hz}, 1 \mathrm{H}, \mathrm{CH}_{\mathrm{Ar}}\right), 7.52(\mathrm{dd}, J=7.7$, $\left.0.8 \mathrm{~Hz}, 1 \mathrm{H}, \mathrm{CH}_{\mathrm{Ar}}\right), 7.49\left(\mathrm{dd}, J=7.9,0.8 \mathrm{~Hz}, 1 \mathrm{H}, \mathrm{CH}_{\mathrm{Ar}}\right), 4.62\left(\mathrm{q}, J=7.1 \mathrm{~Hz}, 2 \mathrm{H}, \mathrm{CH}_{2}\right), 3.86(\mathrm{~s}$, $\left.2 \mathrm{H}, \mathrm{CH}_{2}\right), 3.50\left(\mathrm{~s}, 3 \mathrm{H}, \mathrm{SO}_{2} \mathrm{CH}_{3}\right), 1.42\left(\mathrm{t}, J=7.1 \mathrm{~Hz}, 3 \mathrm{H}, \mathrm{CH}_{3}\right)$.

${ }^{13} \mathrm{C}$ NMR (101 MHz, Chloroform- $\left.d\right) \delta 212.2(\mathrm{C}=\mathrm{S}), 167.3(\mathrm{C}=\mathrm{O}), 151.1\left(\mathrm{C}_{\mathrm{Ar}}\right), 148.4\left(\mathrm{C}_{\mathrm{Ar}}\right)$, 141.2 $\left(\mathrm{CH}_{\mathrm{Ar}}\right), 126.1\left(\mathrm{CH}_{\mathrm{Ar}}\right), 124.7\left(\mathrm{CH}_{\mathrm{Ar}}\right), 71.2\left(\mathrm{CH}_{2}\right), 42.0\left(\mathrm{SO}_{2} \mathrm{CH}_{3}\right), 41.3\left(\mathrm{CH}_{2}\right), 13.7\left(\mathrm{CH}_{3}\right)$.

IR (neat), $v\left(\mathrm{~cm}^{-1}\right): 1717,1578,1561,1427,1357,1228,1146,1044,963,875,772,731$.

HRMS (ESI) m/z: [M + H] ${ }^{+}$Calcd for $\mathrm{C}_{11} \mathrm{H}_{14} \mathrm{ClN}_{2} \mathrm{O}_{4} \mathrm{~S}_{3}$ 368.9799; Found 368.9803.

$\mathrm{R}_{\mathrm{f}}=0.67(\mathrm{PE} / \mathrm{EtOAc}=40 \%)$.

m.p. $=99-100^{\circ} \mathrm{C}$ (crystallized from EtOAc). 
<smiles>CCOC(=S)SC(CCC(=O)N(c1ccc(Cl)nc1)S(C)(=O)=O)COC(C)=O</smiles>

Chemical Formula: $\mathrm{C}_{16} \mathrm{H}_{21} \mathrm{CIN}_{2} \mathrm{O}_{6} \mathrm{~S}_{3}$ Molecular Weight: 468.98

Following the general procedure D, the reaction was carried out with the solution of 3a (304 mg, $0.82 \mathrm{mmol}, 1$ eq.) and allyl acetate $(0.18 \mathrm{~mL}, 1.63 \mathrm{mmol}, 2$ eq.) in EtOAc (0.82 mL) with 0.1 eq. of DLP $(32 \mathrm{mg}$ ). Flash column chromatography on silica gel (eluent: PE/EtOAc $=0 \%$ $\rightarrow 40 \%)$ afforded target product $4 \mathbf{a a}(330 \mathrm{mg}, 85 \%)$ as a yellowish oil.

${ }^{1} \mathrm{H}$ NMR (400 MHz, Chloroform- $d$ ) $\delta 8.30\left(\mathrm{dd}, J=2.8,0.7 \mathrm{~Hz}, 1 \mathrm{H}, \mathrm{CH}_{\mathrm{Ar}}\right), 7.61(\mathrm{dd}, J=8.4$, $\left.2.8 \mathrm{~Hz}, 1 \mathrm{H}, \mathrm{CH}_{\mathrm{Ar}}\right), 7.47\left(\mathrm{dd}, J=8.4,0.7 \mathrm{~Hz}, 1 \mathrm{H}, \mathrm{CH}_{\mathrm{Ar}}\right), 4.64\left(\mathrm{q}, J=7.1 \mathrm{~Hz}, 2 \mathrm{H}, \mathrm{CH}_{2}\right), 4.28$ $\left(\mathrm{dd}, J=11.4,4.9 \mathrm{~Hz}, 1 \mathrm{H}, \mathrm{CH} H_{A l} \mathrm{H}\right), 4.19\left(\mathrm{dd}, J=11.5,6.1 \mathrm{~Hz}, 1 \mathrm{H}, \mathrm{CH} H_{A 2}\right), 4.06-3.91(\mathrm{~m}, 1 \mathrm{H}$, $\mathrm{S}-\mathrm{CH}), 3.50\left(\mathrm{~s}, 3 \mathrm{H}, \mathrm{SO}_{2} \mathrm{CH}_{3}\right), 2.35-2.17\left(\mathrm{~m}, 3 \mathrm{H}, \mathrm{CH}_{2}\right.$ and $\left.\mathrm{CH}_{B 1} \mathrm{H}\right), 2.06\left(\mathrm{~s}, 3 \mathrm{H}, \mathrm{CH}_{3}\right), 1.92$ $-1.80\left(\mathrm{~m}, 1 \mathrm{H}, \mathrm{CH} H_{B 2}\right), 1.43\left(\mathrm{t}, J=7.1 \mathrm{~Hz}, 3 \mathrm{H}, \mathrm{CH}_{3}\right)$.

${ }^{13} \mathrm{C}$ NMR (101 MHz, Chloroform- $\left.d\right) \delta 213.3(\mathrm{C}=\mathrm{S}), 172.3(\mathrm{C}=\mathrm{O}), 170.7(\mathrm{C}=\mathrm{O}), 153.2\left(\mathrm{C}_{\mathrm{Ar}}\right)$, $150.2\left(\mathrm{CH}_{\mathrm{Ar}}\right), 140.1\left(\mathrm{CH}_{\mathrm{Ar}}\right), 131.3\left(\mathrm{C}_{\mathrm{Ar}}\right), 125.7\left(\mathrm{CH}_{\mathrm{Ar}}\right), 71.0\left(\mathrm{CH}_{2}\right), 65.8\left(\mathrm{CH}_{2}\right), 49.0(\mathrm{~S}-\mathrm{CH})$, $42.6\left(\mathrm{SO}_{2} \mathrm{CH}_{3}\right), 34.2\left(\mathrm{CH}_{2}\right), 25.8\left(\mathrm{CH}_{2}\right), 20.9\left(\mathrm{CH}_{3}\right), 13.9\left(\mathrm{CH}_{3}\right)$.

IR (neat), $v\left(\mathrm{~cm}^{-1}\right): 2984,2934,1735,1712,1562,1455,1355,1222,1151,1109,1039,963$, $909,776,731$.

HRMS (ESI) m/z: $[\mathrm{M}+\mathrm{H}]^{+}$Calcd for $\mathrm{C}_{16} \mathrm{H}_{22} \mathrm{ClN}_{2} \mathrm{O}_{6} \mathrm{~S}_{3}$ 469.0323; Found 469.0328.

$\mathrm{R}_{\mathrm{f}}=0.50(\mathrm{PE} / \mathrm{EtOAc}=40 \%)$. 


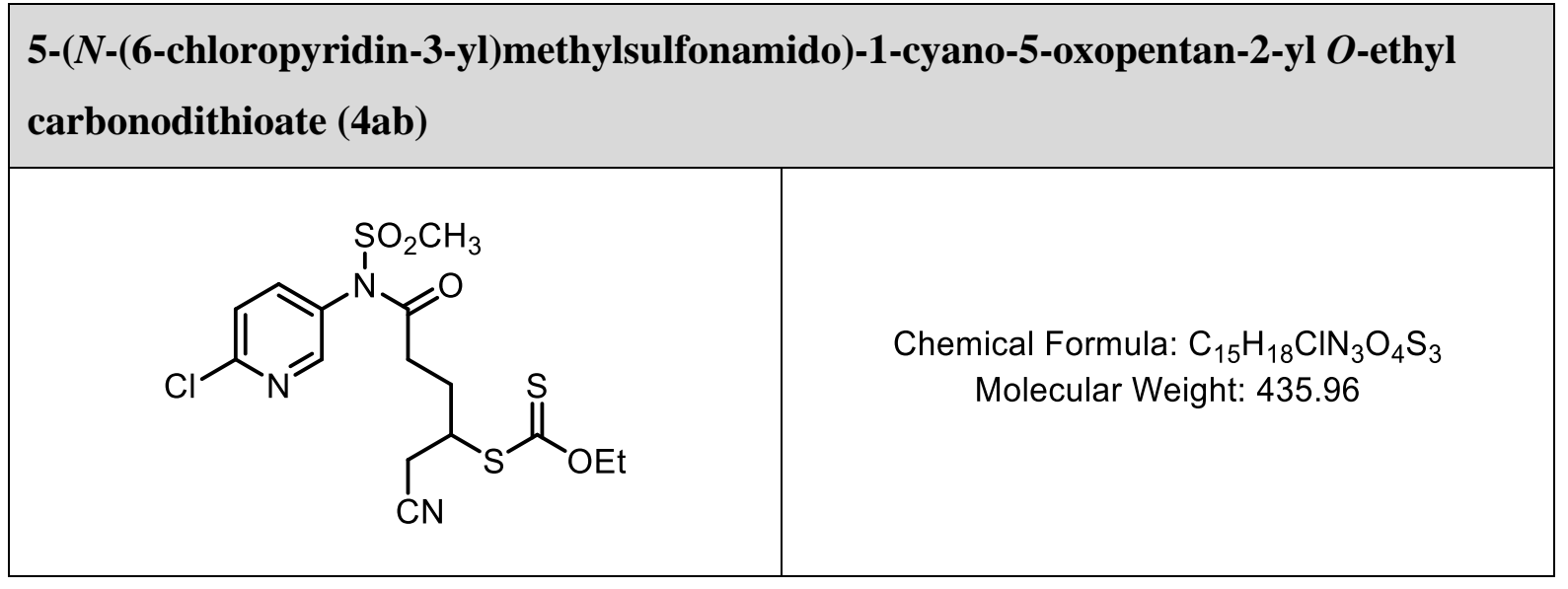

Following the general procedure D, the reaction was carried out with the solution of 3a (300 $\mathrm{mg}, 0.81 \mathrm{mmol}, 1$ eq.) and allyl cyanide $(0.13 \mathrm{~mL}, 1.63 \mathrm{mmol}, 2$ eq. $)$ in EtOAc $(0.82 \mathrm{~mL})$ with 0.2 eq. of DLP (70 mg). Flash column chromatography on silica gel (eluent: PE/EtOAc $=0 \%$ $\rightarrow 50 \%)$ afforded target product $\mathbf{4 a b}(280 \mathrm{mg}, 79 \%)$ as a yellowish oil.

${ }^{1} \mathrm{H}$ NMR (400 MHz, Chloroform- $d$ ) $\delta 8.31\left(\mathrm{dd}, J=2.8,0.7 \mathrm{~Hz}, 1 \mathrm{H}, \mathrm{CH}_{\mathrm{Ar}}\right), 7.63(\mathrm{dd}, J=8.4$, $\left.2.8 \mathrm{~Hz}, 1 \mathrm{H}, \mathrm{CH}_{\mathrm{Ar}}\right), 7.48\left(\mathrm{dd}, J=8.4,0.7 \mathrm{~Hz}, 1 \mathrm{H}, \mathrm{CH}_{\mathrm{Ar}}\right), 4.65\left(\mathrm{qd}, J=7.1,0.7 \mathrm{~Hz}, 2 \mathrm{H}, \mathrm{CH}_{2}\right)$, $3.98-3.89$ (m, 1H, S-CH), 3.49 (s, 3H, $\left.\mathrm{SO}_{2} \mathrm{CH}_{3}\right), 2.89-2.84\left(\mathrm{~m}, 2 \mathrm{H}, \mathrm{CH}_{2}\right), 2.39-2.22(\mathrm{~m}$, $3 \mathrm{H}, \mathrm{CH}_{2}$ and $\left.\mathrm{CH}_{A l} \mathrm{H}\right), 2.06-1.94\left(\mathrm{~m}, 1 \mathrm{H}, \mathrm{CHH}_{A 2}\right), 1.44\left(\mathrm{t}, J=7.1 \mathrm{~Hz}, 3 \mathrm{H}, \mathrm{CH}_{3}\right)$.

${ }^{13} \mathrm{C}$ NMR (101 MHz, Chloroform- $\left.d\right) \delta 212.0(\mathrm{C}=\mathrm{S}), 171.9(\mathrm{C}=\mathrm{O}), 153.2\left(\mathrm{C}_{\mathrm{Ar}}\right), 150.2\left(\mathrm{CH}_{\mathrm{Ar}}\right)$, 140.1 $\left(\mathrm{CH}_{\mathrm{Ar}}\right), 131.2\left(\mathrm{C}_{\mathrm{Ar}}\right), 125.8\left(\mathrm{CH}_{\mathrm{Ar}}\right), 117.0(\mathrm{CN}), 71.2\left(\mathrm{CH}_{2}\right), 45.8(\mathrm{~S}-\mathrm{CH}), 42.6\left(\mathrm{SO}_{2} \mathrm{CH}_{3}\right)$, $34.1\left(\mathrm{CH}_{2}\right), 27.4\left(\mathrm{CH}_{2}\right), 24.4\left(\mathrm{CH}_{2}\right), 13.9\left(\mathrm{CH}_{3}\right)$.

IR (neat), $v\left(\mathrm{~cm}^{-1}\right):$ 2931, 1709, 1562, 1454, 1354, 1224, 1151, 1109, 1043, 962, 910, 776.

HRMS (ESI) m/z: $[\mathrm{M}+\mathrm{H}]^{+}$Calcd for $\mathrm{C}_{15} \mathrm{H}_{19} \mathrm{ClN}_{3} \mathrm{O}_{4} \mathrm{~S}_{3}$ 436.0221; Found 436.0229.

$\mathrm{R}_{\mathrm{f}}=0.26(\mathrm{PE} / \mathrm{EtOAc}=40 \%)$. 


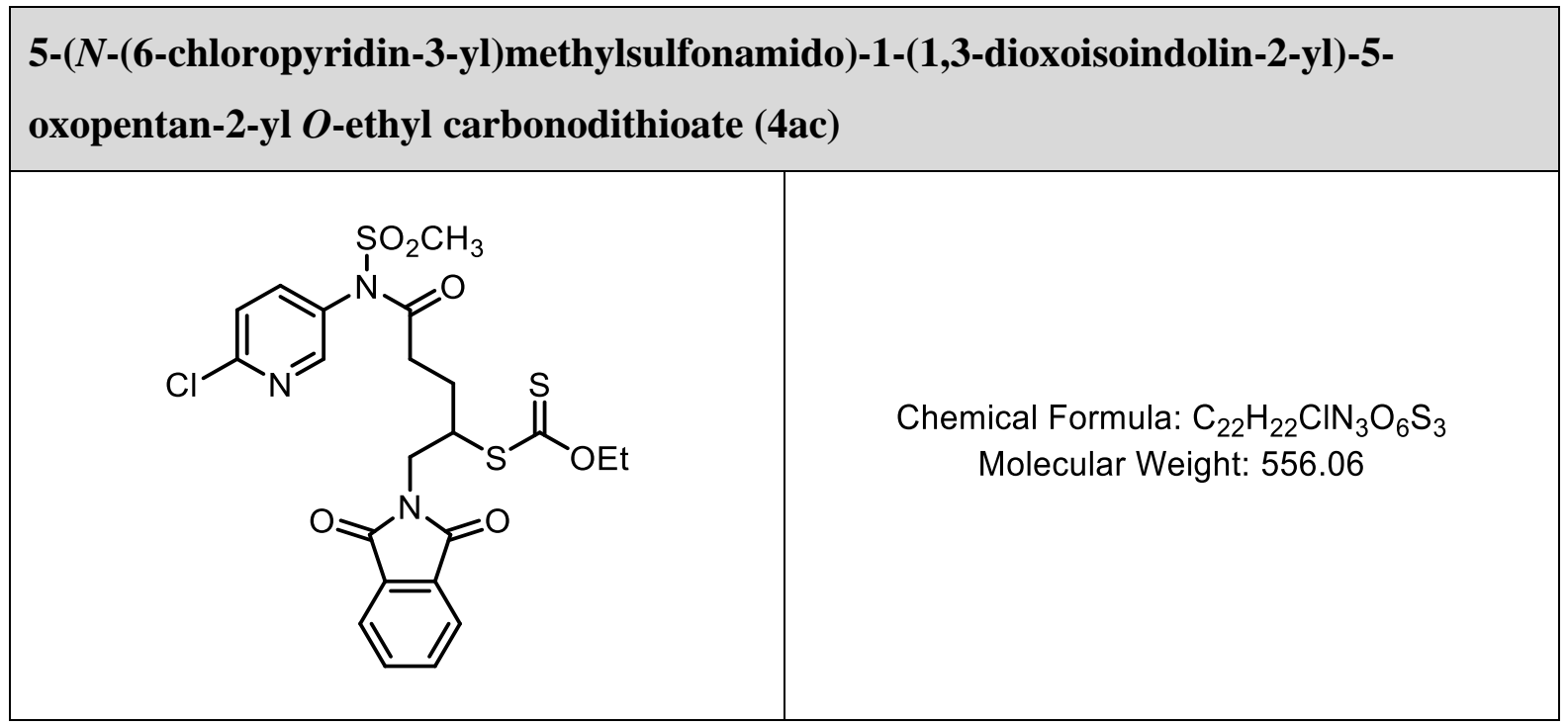

Following the general procedure D, the reaction was carried out with the solution of $\mathbf{3 a}$ (302 $\mathrm{mg}, 0.82 \mathrm{mmol}, 1$ eq.) and $N$-allyl phthalimide (302 mg, $1.63 \mathrm{mmol}, 2$ eq.) in EtOAc (0.82 mL) with 0.1 eq. of DLP (35 mg). Flash column chromatography on silica gel (eluent: PE/EtOAc $=$ $10 \% \rightarrow 40 \% \rightarrow 50 \%)$ afforded target product 4 ac $(367 \mathrm{mg}, 81 \%)$ as a white foam.

${ }^{1} \mathrm{H}$ NMR (400 MHz, Chloroform- $\left.d\right) \delta 8.31\left(\mathrm{dd}, J=2.7,0.7 \mathrm{~Hz}, 1 \mathrm{H}, \mathrm{CH}_{\mathrm{Ar}}\right), 7.86-7.82(\mathrm{~m}, 2 \mathrm{H}$, $\mathrm{CH}_{\text {Phth }}$ ), 7.75 - 7.71 (m, 2H, CHPhth), 7.62 (dd, $J=8.4,2.8 \mathrm{~Hz}, 1 \mathrm{H}, \mathrm{CH}_{\mathrm{Ar}}$ ), 7.46 (dd, $J=8.4$, $\left.0.8 \mathrm{~Hz}, 1 \mathrm{H}, \mathrm{CH}_{\mathrm{Ar}}\right), 4.59$ (q, $\left.J=7.1 \mathrm{~Hz}, 2 \mathrm{H}, \mathrm{CH}_{2}\right), 4.16-4.07$ (m, 1H, S-CH), 3.94 (dd, $J=$ 14.1, 7.2 Hz, $\left.1 \mathrm{H}, \mathrm{CH}_{A l} \mathrm{H}\right), 3.86\left(\mathrm{dd}, J=14.1,7.5 \mathrm{~Hz}, 1 \mathrm{H}, \mathrm{CH} H_{A 2}\right), 3.49$ (s, $\left.3 \mathrm{H}, \mathrm{SO}_{2} \mathrm{CH}_{3}\right), 2.38$ $-2.30\left(\mathrm{~m}, 2 \mathrm{H}, \mathrm{CH}_{2}\right), 2.25-2.12\left(\mathrm{~m}, 1 \mathrm{H}, \mathrm{CH}_{B I} \mathrm{H}\right), 1.93-1.81\left(\mathrm{~m}, 1 \mathrm{H}, \mathrm{CH} H_{B 2}\right), 1.40(\mathrm{t}, J=7.1$ $\left.\mathrm{Hz}, 3 \mathrm{H}, \mathrm{CH}_{3}\right)$.

${ }^{13} \mathrm{C}$ NMR (101 MHz, Chloroform- $\left.d\right) \delta 212.5(\mathrm{C}=\mathrm{S}), 172.2(\mathrm{C}=\mathrm{O}), 168.2(\mathrm{C}=\mathrm{O}), 153.1\left(\mathrm{C}_{\mathrm{Ar}}\right)$, $150.3\left(\mathrm{CH}_{\mathrm{Ar}}\right), 140.2\left(\mathrm{CH}_{\mathrm{Ar}}\right), 134.4(2 \mathrm{C}, \mathrm{CH}$ Phth $), 131.8\left(2 \mathrm{C}, \mathrm{C}_{\mathrm{Phth}}\right), 131.3\left(\mathrm{C}_{\mathrm{Ar}}\right), 125.7\left(\mathrm{CH}_{\mathrm{Ar}}\right)$, 123.7 (2C, $\left.\mathrm{CH}_{\text {Phth }}\right), 70.9\left(\mathrm{CH}_{2}\right), 48.7(\mathrm{~S}-\mathrm{CH}), 42.6\left(\mathrm{SO}_{2} \mathrm{CH}_{3}\right), 40.8\left(\mathrm{CH}_{2}\right), 34.0\left(\mathrm{CH}_{2}\right), 26.2$ $\left(\mathrm{CH}_{2}\right), 13.8\left(\mathrm{CH}_{3}\right)$.

IR (neat), $v\left(\mathrm{~cm}^{-1}\right): 2934,1711,1456,1394,1356,1223,1155,1110,1046,963,777,716$.

HRMS (ESI) m/z: $[\mathrm{M}+\mathrm{H}]^{+}$Calcd for $\mathrm{C}_{22} \mathrm{H}_{23} \mathrm{ClN}_{3} \mathrm{O}_{6} \mathrm{~S}_{3}$ 556.0432; Found 556.0436.

$\mathrm{R}_{\mathrm{f}}=0.27(\mathrm{PE} / \mathrm{EtOAc}=40 \%)$. 


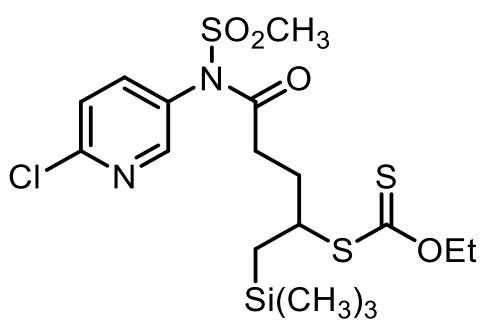

Chemical Formula: $\mathrm{C}_{17} \mathrm{H}_{27} \mathrm{CIN}_{2} \mathrm{O}_{4} \mathrm{~S}_{3} \mathrm{Si}$ Molecular Weight: 483.13

Following the general procedure D, the reaction was carried out with the solution of 3a (304 mg, $0.82 \mathrm{mmol}, 1$ eq.) and allyltrimethylsilane (0.26 mL, $1.63 \mathrm{mmol}, 2$ eq.) in EtOAc (0.82 $\mathrm{mL})$ with 0.2 eq. of DLP $(70 \mathrm{mg})$. Flash column chromatography on silica gel (eluent: $\mathrm{PE} / \mathrm{EtOAc}=0 \% \rightarrow 10 \% \rightarrow 30 \%)$ afforded target product $\mathbf{4 a d}(325 \mathrm{mg}, 82 \%)$ as a colorless oil.

${ }^{1} \mathrm{H}$ NMR (400 MHz, Chloroform- $d$ ) $\delta 8.29\left(\mathrm{dd}, J=2.7,0.7 \mathrm{~Hz}, 1 \mathrm{H}, \mathrm{CH}_{\mathrm{Ar}}\right), 7.59(\mathrm{dd}, J=8.4$, $\left.2.7 \mathrm{~Hz}, 1 \mathrm{H}, \mathrm{CH}_{\mathrm{Ar}}\right), 7.44\left(\mathrm{dd}, J=8.4,0.7 \mathrm{~Hz}, 1 \mathrm{H}, \mathrm{CH}_{\mathrm{Ar}}\right), 4.62\left(\mathrm{qd}, J=7.1,2.8 \mathrm{~Hz}, 2 \mathrm{H}, \mathrm{CH}_{2}\right)$, $3.92-3.82$ (m, 1H, C-S), 3.50 (s, 3H, $\mathrm{SO}_{2} \mathrm{CH}_{3}$ ), $2.31-2.16\left(\mathrm{~m}, 3 \mathrm{H}, \mathrm{CH}_{2}\right.$ and $\left.\mathrm{CH}_{A l} \mathrm{H}\right), 1.82-$ $1.70\left(\mathrm{~m}, 1 \mathrm{H}, \mathrm{CH} H_{A 2}\right), 1.42\left(\mathrm{t}, J=7.1 \mathrm{~Hz}, 3 \mathrm{H}, \mathrm{CH}_{3}\right), 1.08\left(\mathrm{dd}, J=14.9,7.1 \mathrm{~Hz}, 1 \mathrm{H}, \mathrm{CH}_{B 1} \mathrm{H}\right)$, $0.94\left(\mathrm{dd}, J=14.9,8.1 \mathrm{~Hz}, 1 \mathrm{H}, \mathrm{CH} H_{B 2}\right), 0.06\left(\mathrm{~s}, 9 \mathrm{H}, \mathrm{Si}\left(\mathrm{CH}_{3}\right)_{3}\right)$.

${ }^{13} \mathrm{C}$ NMR $(101 \mathrm{MHz}$, Chloroform- $d) \delta 215.0(\mathrm{C}=\mathrm{S}), 172.6(\mathrm{C}=\mathrm{O}), 153.0\left(\mathrm{C}_{\mathrm{Ar}}\right), 150.3\left(\mathrm{CH}_{\mathrm{Ar}}\right)$, 140.2 $\left(\mathrm{CH}_{\mathrm{Ar}}\right), 131.4\left(\mathrm{C}_{\mathrm{Ar}}\right), 125.6\left(\mathrm{CH}_{\mathrm{Ar}}\right), 70.3\left(\mathrm{CH}_{2}\right), 48.4(\mathrm{~S}-\mathrm{CH}), 42.6\left(\mathrm{SO}_{2} \mathrm{CH}_{3}\right), 34.5\left(\mathrm{CH}_{2}\right)$, 32.1 $\left(\mathrm{CH}_{2}\right), 24.2\left(\mathrm{CH}_{2}\right), 14.0\left(\mathrm{CH}_{3}\right),-0.7\left(3 \mathrm{C}, \mathrm{Si}\left(\mathrm{CH}_{3}\right)_{3}\right)$.

IR (neat), $v\left(\mathrm{~cm}^{-1}\right): 2947,1711,1562,1455,1356,1248,1212,1153,1109,1046,963,845$, $776,732$.

HRMS (ESI) m/z: $[\mathrm{M}+\mathrm{H}]^{+}$Calcd for $\mathrm{C}_{17} \mathrm{H}_{28} \mathrm{ClN}_{2} \mathrm{O}_{4} \mathrm{~S}_{3} \mathrm{Si} 483.0664$; Found 483.0682.

$\mathrm{R}_{\mathrm{f}}=0.68(\mathrm{PE} / \mathrm{EtOAc}=40 \%)$. 


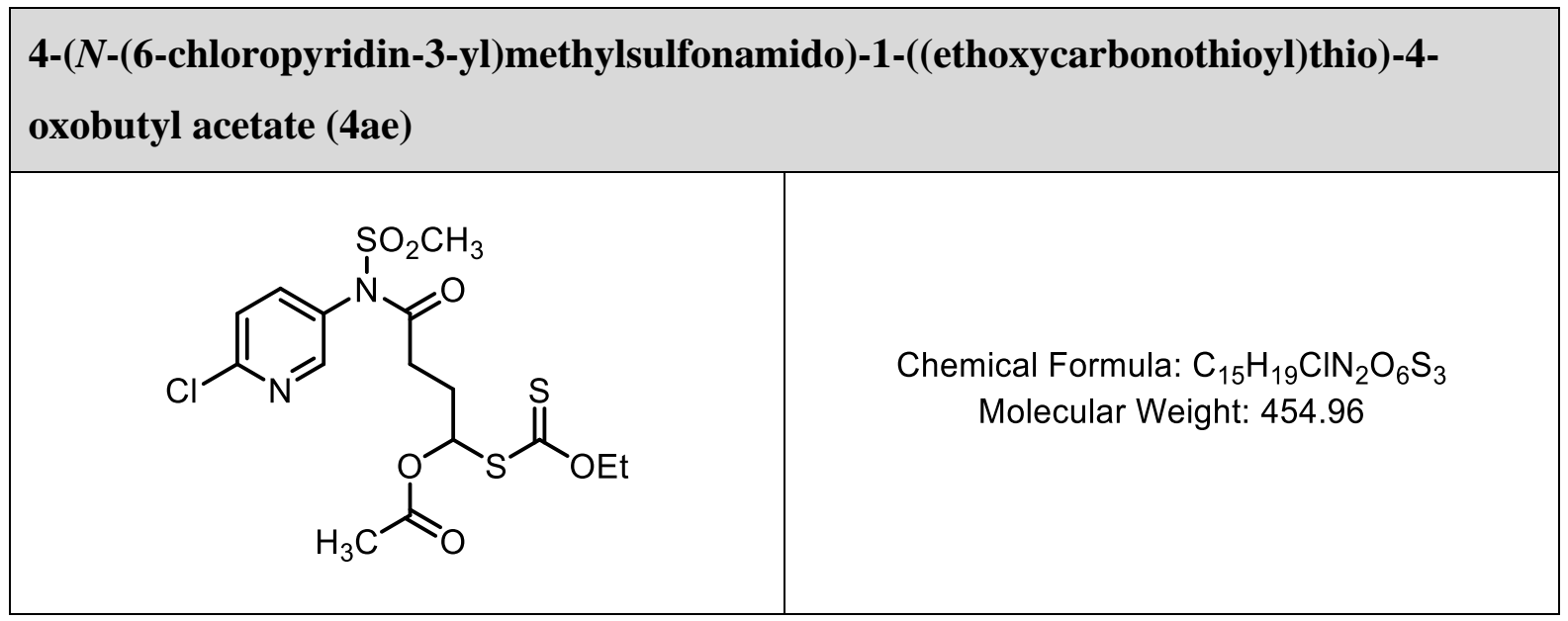

Following the general procedure D, the reaction was carried out with the solution of 3a (304 $\mathrm{mg}, 0.82 \mathrm{mmol}, 1$ eq.) and vinyl acetate $(0.15 \mathrm{~mL}, 1.63 \mathrm{mmol}, 2$ eq.) in EtOAc (0.82 mL) with 0.2 eq. of DLP $(70 \mathrm{mg})$. Flash column chromatography on silica gel (eluent: PE/EtOAc $=0 \%$ $\rightarrow 10 \% \rightarrow 40 \%)$ afforded target product $4 \mathbf{a e}(278 \mathrm{mg}, 74 \%)$ as a colorless oil.

${ }^{1} \mathrm{H}$ NMR (400 MHz, Chloroform- $d$ ) $\delta 8.33\left(\mathrm{dd}, J=2.8,0.7 \mathrm{~Hz}, 1 \mathrm{H}, \mathrm{CH}_{\mathrm{Ar}}\right), 7.64(\mathrm{dd}, J=8.4$, $\left.2.8 \mathrm{~Hz}, 1 \mathrm{H}, \mathrm{CH}_{\mathrm{Ar}}\right), 7.48\left(\mathrm{dd}, J=8.4,0.7 \mathrm{~Hz}, 1 \mathrm{H}, \mathrm{CH}_{\mathrm{Ar}}\right), 6.59$ (dd, $\left.J=7.1,5.2 \mathrm{~Hz}, 1 \mathrm{H}, \mathrm{S}-\mathrm{CH}\right)$, $4.69-4.55\left(\mathrm{~m}, 2 \mathrm{H}, \mathrm{OCH}_{2}\right), 3.51\left(\mathrm{~s}, 3 \mathrm{H}, \mathrm{SO}_{2} \mathrm{CH}_{3}\right), 2.41-2.11\left(\mathrm{~m}, 4 \mathrm{H}, 2 \times \mathrm{CH}_{2}\right), 1.40(\mathrm{t}, J=$ $\left.7.1 \mathrm{~Hz}, 3 \mathrm{H}, \mathrm{CH}_{3}\right)$.

${ }^{13} \mathrm{C}$ NMR (101 MHz, Chloroform- $\left.d\right) \delta 209.8(\mathrm{C}=\mathrm{S}), 171.5(\mathrm{C}=\mathrm{O}), 170.1(\mathrm{C}=\mathrm{O}), 153.2\left(\mathrm{C}_{\mathrm{Ar}}\right)$, $150.3\left(\mathrm{CH}_{\mathrm{Ar}}\right), 140.2\left(\mathrm{CH}_{\mathrm{Ar}}\right), 131.3\left(\mathrm{C}_{\mathrm{Ar}}\right), 125.7\left(\mathrm{CH}_{\mathrm{Ar}}\right), 79.8(\mathrm{~S}-\mathrm{CH}), 70.7\left(\mathrm{CH}_{2}\right), 42.5$ $\left(\mathrm{SO}_{2} \mathrm{CH}_{3}\right), 32.8\left(\mathrm{CH}_{2}\right), 28.5\left(\mathrm{CH}_{2}\right), 21.0\left(\mathrm{CH}_{3}\right), 13.8\left(\mathrm{CH}_{3}\right)$.

IR (neat), $v\left(\mathrm{~cm}^{-1}\right): 2986,2935,1742,1712,1562,1455,1358,1223,1155,1110,1049,1027$, 962, 909, 777, 733 .

HRMS (ESI) m/z: $[\mathrm{M}+\mathrm{H}]^{+}$Calcd for $\mathrm{C}_{15} \mathrm{H}_{20} \mathrm{ClN}_{2} \mathrm{O}_{6} \mathrm{~S}_{3}$ 455.0167; Found 455.0178.

$\mathrm{R}_{\mathrm{f}}=0.47(\mathrm{PE} / \mathrm{EtOAc}=40 \%)$. 


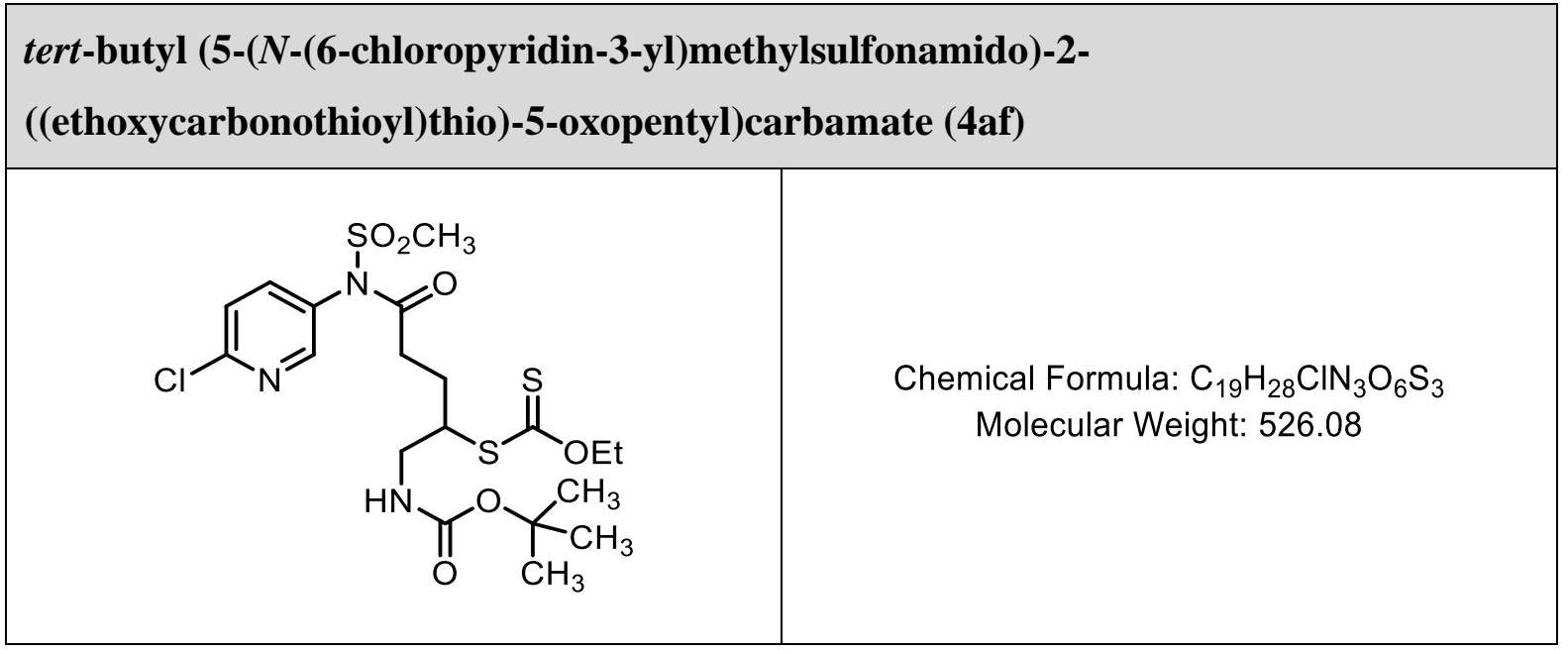

Following the general procedure D, the reaction was carried out with the solution of $\mathbf{3 a}$ (300 $\mathrm{mg}, 0.81 \mathrm{mmol}, 1$ eq.) and tert-butyl allylcarbamate (145 mg, $0.92 \mathrm{mmol}, 1.1$ eq.) in EtOAc $(0.82 \mathrm{~mL})$ with 0.3 eq. of DLP (105 mg). Flash column chromatography on silica gel (eluent: $\mathrm{PE} / \mathrm{EtOAc}=0 \% \rightarrow 50 \%)$ afforded target product $4 \mathbf{a f}(268 \mathrm{mg}, 63 \%)$ as a colorless oil. Product contained traces of the reduced starting material ( $N$-(6-chloropyridin-3-yl)- $N$ (methylsulfonyl)acetamide) inseparable by column chromatography.

${ }^{1} \mathrm{H}$ NMR (400 MHz, Chloroform- $d$ ) $\delta 8.30\left(\mathrm{dd}, J=2.7,0.7 \mathrm{~Hz}, 1 \mathrm{H}, \mathrm{CH}_{\mathrm{Ar}}\right), 7.62(\mathrm{dd}, J=8.4$, $\left.2.7 \mathrm{~Hz}, 1 \mathrm{H}, \mathrm{CH}_{\mathrm{Ar}}\right), 7.45\left(\mathrm{dd}, J=8.4,0.7 \mathrm{~Hz}, 1 \mathrm{H}, \mathrm{CH}_{\mathrm{Ar}}\right), 4.84$ (t, br, $\left.J=5.4 \mathrm{~Hz}, 1 \mathrm{H}, \mathrm{NH}\right), 4.62$ (qd, $\left.J=7.1,0.8 \mathrm{~Hz}, 2 \mathrm{H}, \mathrm{CH}_{2}\right), 3.85-3.76(\mathrm{~m}, 1 \mathrm{H}, \mathrm{S}-\mathrm{CH}), 3.48\left(\mathrm{~s}, 3 \mathrm{H}, \mathrm{SO}_{2} \mathrm{CH}_{3}\right), 3.45-3.36$ $\left(\mathrm{m}, 1 \mathrm{H}, \mathrm{CH}_{A 1} \mathrm{H}\right), 3.35-3.23\left(\mathrm{~m}, 1 \mathrm{H}, \mathrm{CH} H_{A 2}\right), 2.37-2.26\left(\mathrm{~m}, 2 \mathrm{H}, \mathrm{CH}_{2}\right), 2.22-2.09(\mathrm{~m}, 1 \mathrm{H}$, $\left.\mathrm{CH}_{B l} \mathrm{H}\right), 1.88-1.76\left(\mathrm{~m}, 1 \mathrm{H}, \mathrm{CH}_{B 2}\right), 1.45-1.38\left(\mathrm{~m}, 12 \mathrm{H}, 4 \mathrm{x} \mathrm{CH}_{3}\right)$.

${ }^{13} \mathrm{C}$ NMR (101 MHz, Chloroform- $\left.d\right) \delta 213.5(\mathrm{C}=\mathrm{S}), 172.4(\mathrm{C}=\mathrm{O}), 156.0(\mathrm{C}=\mathrm{O}), 153.0\left(\mathrm{C}_{\mathrm{Ar}}\right)$, 150.2 $\left(\mathrm{CH}_{\mathrm{Ar}}\right), 140.2\left(\mathrm{C}_{\mathrm{Ar}}\right), 131.4\left(\mathrm{CH}_{\mathrm{Ar}}\right), 125.6\left(\mathrm{C}_{\mathrm{Ar}}\right), 79.9(\mathrm{C}), 70.8\left(\mathrm{CH}_{2}\right), 51.1(\mathrm{~S}-\mathrm{CH}), 43.6$ $\left(\mathrm{CH}_{2}\right), 42.5\left(\mathrm{SO}_{2} \mathrm{CH}_{3}\right), 34.2\left(\mathrm{CH}_{2}\right), 28.4\left(3 \mathrm{C}, \mathrm{CH}_{3}\right), 26.0\left(\mathrm{CH}_{2}\right), 13.9\left(\mathrm{CH}_{3}\right)$.

IR (neat), $v\left(\mathrm{~cm}^{-1}\right): 3389,2978,2931,1705,1512,1455,1359,1221,1157,1111,1047,965$, $777,733,549$.

HRMS (ESI) m/z: $[\mathrm{M}+\mathrm{H}]^{+}$Calcd for $\mathrm{C}_{19} \mathrm{H}_{29} \mathrm{ClN}_{3} \mathrm{O}_{6} \mathrm{~S}_{3}$ 526.0902; Found 526.0915.

$\mathrm{R}_{\mathrm{f}}=0.62(\mathrm{PE} / \mathrm{EtOAc}=40 \%)$. 


\section{5-(N-(6-chloropyridin-3-yl)methylsulfonamido)-5-oxo-1-(4,4,5,5-tetramethyl-1,3,2- dioxaborolan-2-yl)pentan-2-yl $O$-ethyl carbonodithioate (4ag)}<smiles>CCOC(=S)SC(CCC(=O)N(c1ccc(Cl)nc1)S(C)(=O)=O)CB1OC(C)(C)C(C)(C)O1</smiles>

Chemical Formula: $\mathrm{C}_{20} \mathrm{H}_{30} \mathrm{BCIN}_{2} \mathrm{O}_{6} \mathrm{~S}_{3}$ Molecular Weight: 536.91

Following the general procedure D, the reaction was carried out with the solution of 3a (300 $\mathrm{mg}, 0.81 \mathrm{mmol}, 1$ eq.) and 2-allyl-4,4,5,5-tetramethyl-1,3,2-dioxaborolane (0.31 mL, 1.63 mmol, 2 eq. $)$ in EtOAc $(0.82 \mathrm{~mL})$ with 0.2 eq. of DLP $(70 \mathrm{mg})$. Flash column chromatography on silica gel (eluent: $\mathrm{PE} / \mathrm{EtOAc}=0 \% \rightarrow 40 \%$ ) afforded target product 4ag (316 mg, 72\%) as a colorless oil.

${ }^{1} \mathrm{H}$ NMR (400 MHz, Chloroform- $d$ ) $\delta 8.30\left(\mathrm{dd}, J=2.8,0.7 \mathrm{~Hz}, 1 \mathrm{H}, \mathrm{CH}_{\mathrm{Ar}}\right.$ ), 7.59 (dd, $J=8.4$, $\left.2.8 \mathrm{~Hz}, 1 \mathrm{H}, \mathrm{CH}_{\mathrm{Ar}}\right), 7.45\left(\mathrm{dd}, J=8.4,0.7 \mathrm{~Hz}, 1 \mathrm{H}, \mathrm{CH}_{\mathrm{Ar}}\right), 4.61\left(\mathrm{qd}, J=7.1,1.3 \mathrm{~Hz}, 2 \mathrm{H}, \mathrm{CH}_{2}\right)$, $3.94-3.85$ (m, 1H, S-CH), 3.49 (s, 3H, $\left.\mathrm{SO}_{2} \mathrm{CH}_{3}\right), 2.32-2.07\left(\mathrm{~m}, 3 \mathrm{H}, \mathrm{CH}_{2}\right.$ and $\left.\mathrm{CH}_{A l} \mathrm{H}\right), 1.99$ - $1.86\left(\mathrm{~m}, 1 \mathrm{H}, \mathrm{CH} H_{A 2}\right), 1.40\left(\mathrm{t}, J=7.1 \mathrm{~Hz}, 3 \mathrm{H}, \mathrm{CH}_{3}\right), 1.24-1.20\left(\mathrm{~m}, 2 \mathrm{H}, \mathrm{CH}_{2}-\mathrm{B}\right), 1.23(\mathrm{~s}, 6 \mathrm{H}$, $\left.2 \times \mathrm{CH}_{3}\right), 1.21\left(\mathrm{~s}, 6 \mathrm{H}, 2 \times \mathrm{CH}_{3}\right)$.

${ }^{13} \mathrm{C}$ NMR (101 MHz, Chloroform- $\left.d\right) \delta 214.7(\mathrm{C}=\mathrm{S}), 172.7(\mathrm{C}=\mathrm{O}), 153.0\left(\mathrm{C}_{\mathrm{Ar}}\right), 150.3\left(\mathrm{CH}_{\mathrm{Ar}}\right)$, $140.2\left(\mathrm{CH}_{\mathrm{Ar}}\right), 131.5\left(\mathrm{C}_{\mathrm{Ar}}\right), 125.6\left(\mathrm{CH}_{\mathrm{Ar}}\right), 83.8(2 \mathrm{C}, \mathrm{C}-\mathrm{O}), 70.2\left(\mathrm{CH}_{2}\right), 47.3(\mathrm{~S}-\mathrm{CH}), 42.6$ $\left(\mathrm{SO}_{2} \mathrm{CH}_{3}\right), 34.7\left(\mathrm{CH}_{2}\right), 31.0\left(\mathrm{CH}_{2}\right), 24.9\left(2 \mathrm{C}, \mathrm{CH}_{3}\right), 24.9\left(2 \mathrm{C}, \mathrm{CH}_{3}\right), 13.9\left(\mathrm{CH}_{3}\right) . \mathrm{B}-\mathrm{CH}_{2}$ signal is not observed.

IR (neat), $v\left(\mathrm{~cm}^{-1}\right): 2924,2853,1712,1562,1455,1359,1214,1142,1109,1048,965,845$, $776,735$.

HRMS (ESI) m/z: [M + H $]^{+}$Calcd for $\mathrm{C}_{20} \mathrm{H}_{31} \mathrm{BClN}_{2} \mathrm{O}_{6} \mathrm{~S}_{3}$ 537.1120; Found 537.1138.

$\mathrm{R}_{\mathrm{f}}=0.57(\mathrm{PE} / \mathrm{EtOAc}=40 \%)$. 
S-(6-(N-(6-chloropyridin-3-yl)methylsulfonamido)-6-oxo-1,1-bis $(4,4,5,5$-tetramethyl-

1,3,2-dioxaborolan-2-yl)hexan-3-yl) O-ethyl carbonodithioate (4ah)<smiles>CCOC(=S)SC(CCC(=O)N(c1ccc(Cl)nc1)S(C)(=O)=O)CC(B1OC(C)(C)C(C)(C)O1)B1OC(C)(C)C(C)(C)O1</smiles>

Chemical Formula: $\mathrm{C}_{27} \mathrm{H}_{43} \mathrm{~B}_{2} \mathrm{ClN}_{2} \mathrm{O}_{8} \mathrm{~S}_{3}$ Molecular Weight: 676.90

Following the general procedure D, the reaction was carried out with the solution of $\mathbf{3 a}$ (300 $\mathrm{mg}, 0.81 \mathrm{mmol}, 1$ eq.) and 4,4,5,5-tetramethyl-2-(1-(4,4,5,5-tetramethyl-1,3,2-dioxaborolan-2yl)but-3-en-1-yl)-1,3,2-dioxaborolane ${ }^{2}$ (278 mg, $0.90 \mathrm{mmol}, 1.1$ eq.) in EtOAc (0.82 mL) with 0.4 eq. of DLP (140 mg). Flash column chromatography on silica gel (eluent: PE/EtOAc $=0 \%$ $\rightarrow 30 \%)$ afforded target product $\mathbf{4 a h}(347 \mathrm{mg}, 63 \%)$ as a white foam.

${ }^{1} \mathrm{H}$ NMR (400 MHz, Chloroform-d) $\delta 8.29\left(\mathrm{dd}, \mathrm{J}=2.8,0.7 \mathrm{~Hz}, 1 \mathrm{H}, \mathrm{CH}_{\mathrm{Ar}}\right), 7.59(\mathrm{dd}, \mathrm{J}=8.4$, $\left.2.8 \mathrm{~Hz}, 1 \mathrm{H}, \mathrm{CH}_{\mathrm{Ar}}\right), 7.44\left(\mathrm{dd}, \mathrm{J}=8.4,0.7 \mathrm{~Hz}, 1 \mathrm{H}, \mathrm{CH}_{\mathrm{Ar}}\right), 4.58\left(\mathrm{q}, \mathrm{J}=7.2 \mathrm{~Hz}, 1 \mathrm{H}, \mathrm{CH}_{2}\right), 3.71-$ $3.61(\mathrm{~m}, 1 \mathrm{H}, \mathrm{S}-\mathrm{CH}), 3.48\left(\mathrm{~s}, 3 \mathrm{H}, \mathrm{SO}_{2} \mathrm{CH}_{3}\right), 2.37-2.11\left(\mathrm{~m}, 3 \mathrm{H}, \mathrm{CH}_{2}\right.$ and $\left.\mathrm{CH}_{A l} \mathrm{H}\right), 1.99-1.87$ $\left(\mathrm{m}, 1 \mathrm{H}, \mathrm{CH}_{B 1} \mathrm{H}\right), 1.86-1.69\left(\mathrm{~m}, 2 \mathrm{H}, \mathrm{CH}_{A 2}\right.$ and $\left.\mathrm{CH}_{B 2}\right), 1.39\left(\mathrm{t}, \mathrm{J}=7.1 \mathrm{~Hz}, 3 \mathrm{H}, \mathrm{CH}_{3}\right), 1.21$ (s, 12H, 4 x CH 3$), 1.20-1.19(\mathrm{~m}, 12 \mathrm{H}, 4$ x CH 3$), 0.75(\mathrm{dd}, \mathrm{J}=9.1,6.6 \mathrm{~Hz}, 1 \mathrm{H}, \mathrm{B}-\mathrm{CH})$.

${ }^{13} \mathrm{C}$ NMR $(101 \mathrm{MHz}$, Chloroform- $d) \delta 215.1(\mathrm{C}=\mathrm{S}), 172.6(\mathrm{C}=\mathrm{O}), 152.9\left(\mathrm{C}_{\mathrm{Ar}}\right), 150.3\left(\mathrm{CH}_{\mathrm{Ar}}\right)$, $140.2\left(\mathrm{CH}_{\mathrm{Ar}}\right), 131.5\left(\mathrm{C}_{\mathrm{Ar}}\right), 125.5\left(\mathrm{CH}_{\mathrm{Ar}}\right), 83.4(4 \mathrm{C}, \mathrm{C}-\mathrm{O}), 70.3\left(\mathrm{CH}_{2}\right), 53.7(\mathrm{~S}-\mathrm{CH}), 42.6$ $\left(\mathrm{SO}_{2} \mathrm{CH}_{3}\right), 34.4\left(\mathrm{CH}_{2}\right), 30.2\left(\mathrm{CH}_{2}\right), 29.9\left(\mathrm{CH}_{2}\right), 25.0\left(2 \mathrm{C}, \mathrm{CH}_{3}\right), 25.0\left(2 \mathrm{C}, \mathrm{CH}_{3}\right), 24.7(2 \mathrm{C}$, $\left.\mathrm{CH}_{3}\right), 24.7\left(2 \mathrm{C}, \mathrm{CH}_{3}\right), 13.9\left(\mathrm{CH}_{3}\right)$. B-CH signal is not observed.

IR (neat), $v\left(\mathrm{~cm}^{-1}\right): 2978,2931,1713,1455,1359,1314,1266,1215,1133,1110,1049,966$, $848,776,734$.

HRMS (ESI) m/z: $[\mathrm{M}+\mathrm{H}]^{+}$Calcd for $\mathrm{C}_{27} \mathrm{H}_{44} \mathrm{~B}_{2} \mathrm{ClN}_{2} \mathrm{O}_{8} \mathrm{~S}_{3}$ 677.2129; Found 677.2145.

$\mathrm{R}_{\mathrm{f}}=0.55(\mathrm{PE} / \mathrm{EtOAc}=40 \%)$. 


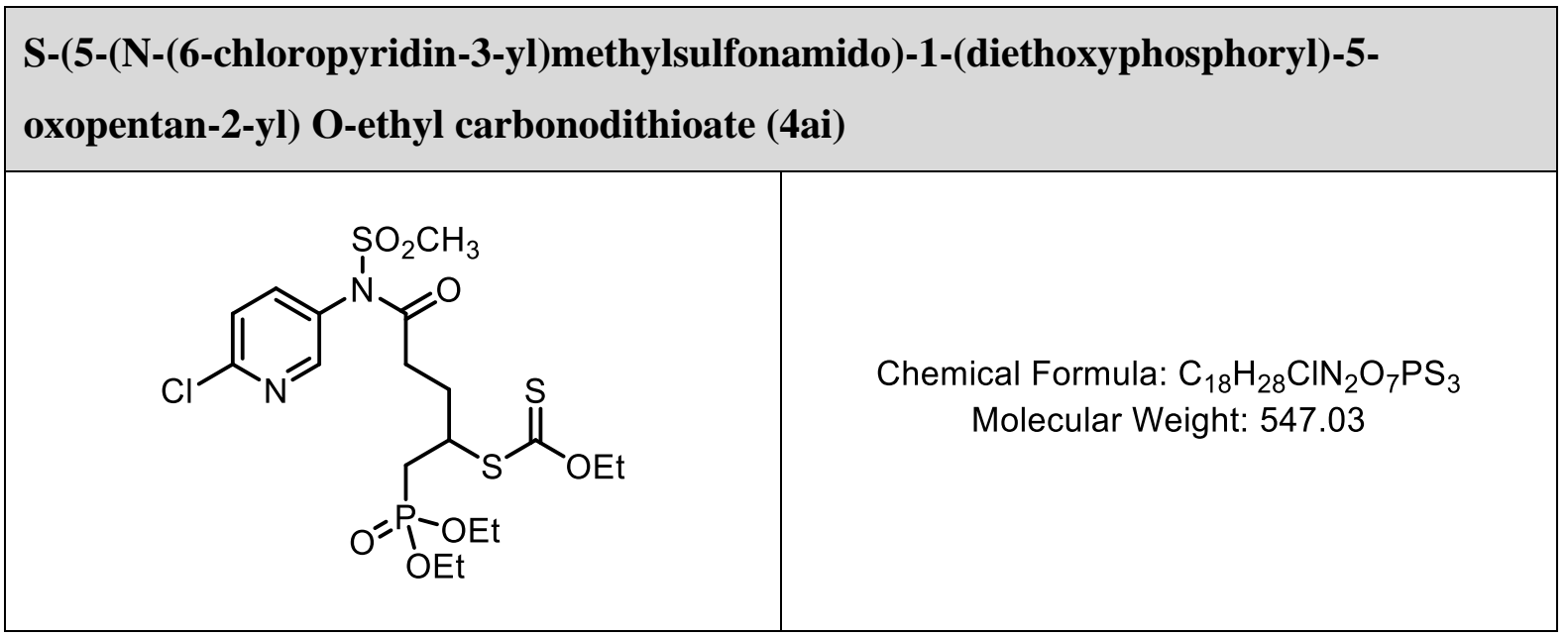

Following the general procedure D, the reaction was carried out with the solution of $\mathbf{3 a}$ (300 $\mathrm{mg}, 0.81 \mathrm{mmol}, 1$ eq.) and diethyl allylphosphonate (160 mg, $0.90 \mathrm{mmol}, 1.1$ eq.) in EtOAc $(0.82 \mathrm{~mL})$ with 0.2 eq. of DLP $(70 \mathrm{mg})$. Then $\mathrm{N}_{2}$ was flushed at heating to $130^{\circ} \mathrm{C}$ during 30 min. Flash column chromatography on silica gel (eluent: PE/EtOAc $=80 \% \rightarrow 100 \%$ ) afforded target product 4ai (308 mg, 69\%) as a colorless oil.

${ }^{1} \mathrm{H}$ NMR (400 MHz, Chloroform- $d$ ) $\delta 8.30\left(\mathrm{dd}, J=2.8,0.7 \mathrm{~Hz}, 1 \mathrm{H}, \mathrm{CH}_{\mathrm{Ar}}\right), 7.61(\mathrm{dd}, J=8.4$, $\left.2.8 \mathrm{~Hz}, 1 \mathrm{H}, \mathrm{CH}_{\mathrm{Ar}}\right), 7.44\left(\mathrm{dd}, J=8.4,0.7 \mathrm{~Hz}, 1 \mathrm{H}, \mathrm{CH}_{\mathrm{Ar}}\right), 4.60\left(\mathrm{qd}, J=7.1,2.0 \mathrm{~Hz}, 2 \mathrm{H}, \mathrm{CH}_{2}\right)$, $4.15-4.01$ (m, 4H, 2 x CH ), $4.01-3.89$ (m, 1H, S-CH), 3.46 (s, 3H, $\left.\mathrm{SO}_{2} \mathrm{CH}_{3}\right), 2.49-2.21$ $\left(\mathrm{m}, 4 \mathrm{H}, \mathrm{CH}_{A 1} \mathrm{H}, \mathrm{CH}_{B 1} \mathrm{H}, \mathrm{CH}_{2}\right), 2.12-1.86\left(\mathrm{~m}, 2 \mathrm{H}, \mathrm{CH} H_{A 2}\right.$ and $\left.\mathrm{CH}_{B 2}\right), 1.39(\mathrm{t}, J=7.1 \mathrm{~Hz}$, $\left.3 \mathrm{H}, \mathrm{CH}_{3}\right), 1.34-1.27\left(\mathrm{~m}, 6 \mathrm{H}, 2 \times \mathrm{CH}_{3}\right)$.

${ }^{13} \mathrm{C}$ NMR $(101 \mathrm{MHz}$, Chloroform- $d) \delta 212.9(\mathrm{C}=\mathrm{S}), 172.2(\mathrm{C}=\mathrm{O}), 152.9\left(\mathrm{C}_{\mathrm{Ar}}\right), 150.3\left(\mathrm{CH}_{\mathrm{Ar}}\right)$, 140.2 $\left(\mathrm{CH}_{\mathrm{Ar}}\right), 131.4\left(\mathrm{C}_{\mathrm{Ar}}\right), 125.6\left(\mathrm{CH}_{\mathrm{Ar}}\right), 70.5\left(\mathrm{CH}_{2}\right), 62.5-61.2\left(\mathrm{~m}, 2 \mathrm{C}, \mathrm{CH}_{2}\right), 44.9(\mathrm{~d}, J=1.9$ $\mathrm{Hz}, \mathrm{S}-\mathrm{CH}), 42.5\left(\mathrm{SO}_{2} \mathrm{CH}_{3}\right), 34.4\left(\mathrm{CH}_{2}\right), 31.9$ (d, $\left.J=137.5 \mathrm{~Hz}, \mathrm{CH}_{2}\right), 28.7$ (d, J=4.3 Hz, $\left.\mathrm{CH}_{2}\right)$, $16.7-16.1\left(\mathrm{~m}, 2 \mathrm{C}, \mathrm{CH}_{3}\right), 13.8\left(\mathrm{CH}_{3}\right)$.

IR (neat), $v\left(\mathrm{~cm}^{-1}\right):$ 2983, 1710, 1563, 1455, 1354, 1223, 1154, 1109, 1040, 959, 775, 734.

HRMS (ESI) m/z: [M + H $]^{+}$Calcd for $\mathrm{C}_{18} \mathrm{H}_{29} \mathrm{ClN}_{2} \mathrm{O}_{7} \mathrm{PS}_{3}$ 547.0558; Found 547.0574.

$\mathrm{R}_{\mathrm{f}}=0.61($ EtOAc) 


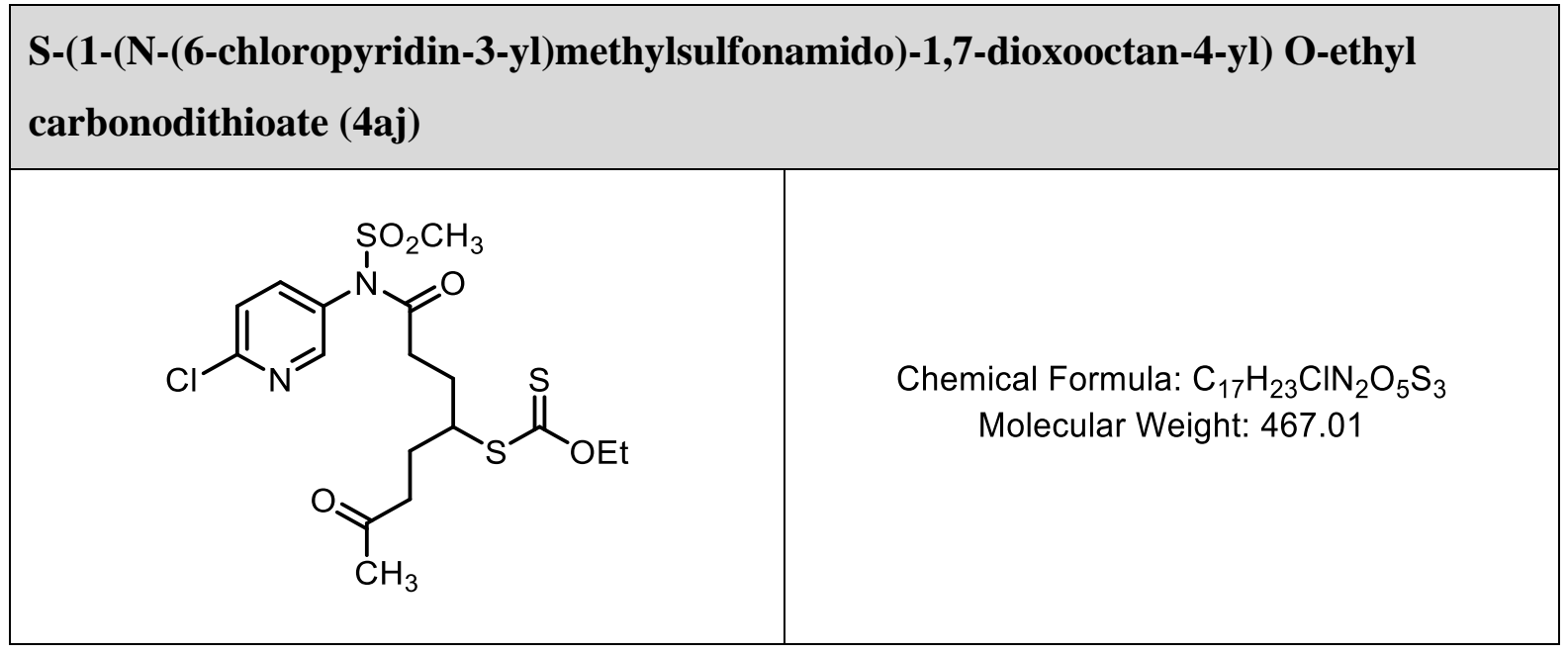

Following the general procedure D, the reaction was carried out with the solution of 3a (304 $\mathrm{mg}, 0.82 \mathrm{mmol}, 1$ eq.) and 5-hexen-2-one (0.19 mL $1.63 \mathrm{mmol}, 2$ eq.) in EtOAc (0.82 mL) with 0.2 eq. of DLP $(70 \mathrm{mg})$. Flash column chromatography on silica gel (eluent: PE/EtOAc $=$ $0 \% \rightarrow 40 \%$ ) afforded target product $\mathbf{4 a j}$ (306 $\mathrm{mg}, 80 \%$ ) as a colorless oil.

${ }^{1} \mathrm{H}$ NMR (400 MHz, Chloroform- $d$ ) $\delta 8.30\left(\mathrm{dd}, J=2.7,0.7 \mathrm{~Hz}, 1 \mathrm{H}, \mathrm{CH}_{\mathrm{Ar}}\right), 7.61(\mathrm{dd}, J=8.4$, $\left.2.7 \mathrm{~Hz}, 1 \mathrm{H}, \mathrm{CH}_{\mathrm{Ar}}\right), 7.45\left(\mathrm{dd}, J=8.4,0.7 \mathrm{~Hz}, 1 \mathrm{H}, \mathrm{CH}_{\mathrm{Ar}}\right), 4.62\left(\mathrm{q}, J=7.1 \mathrm{~Hz}, 2 \mathrm{H}, \mathrm{CH}_{2}\right), 3.75-$ $3.66(\mathrm{~m}, 1 \mathrm{H}, \mathrm{S}-\mathrm{CH}), 3.49$ (s, 3H, SO $\left.\mathrm{CH}_{3}\right), 2.58\left(\mathrm{t}, J=7.2 \mathrm{~Hz}, 2 \mathrm{H}, \mathrm{CH}_{2}\right), 2.31-2.24(\mathrm{~m}, 2 \mathrm{H}$, $\left.\mathrm{CH}_{2}\right), 2.21-2.06\left(\mathrm{~m}, 1 \mathrm{H}, \mathrm{CH}_{A l} \mathrm{H}\right), 2.12\left(\mathrm{~s}, 3 \mathrm{H}, \mathrm{CH}_{3}\right), 2.06-1.92\left(\mathrm{~m}, 1 \mathrm{H}, \mathrm{CH}_{B I} \mathrm{H}\right), 1.89-1.71$ $\left(\mathrm{m}, 2 \mathrm{H}, \mathrm{CH}_{A 2}\right.$ and $\left.\mathrm{CH}_{B 2}\right), 1.42\left(\mathrm{t}, J=7.1 \mathrm{~Hz}, 3 \mathrm{H}, \mathrm{CH}_{3}\right)$.

${ }^{13} \mathrm{C}$ NMR (101 MHz, Chloroform- $\left.d\right) \delta 214.6(\mathrm{C}=\mathrm{S}), 207.6(\mathrm{C}=\mathrm{O}), 172.5(\mathrm{C}=\mathrm{O}), 153.0\left(\mathrm{C}_{\mathrm{Ar}}\right)$, $150.3\left(\mathrm{CH}_{\mathrm{Ar}}\right), 140.2\left(\mathrm{CH}_{\mathrm{Ar}}\right), 131.4\left(\mathrm{C}_{\mathrm{Ar}}\right), 125.6\left(\mathrm{CH}_{\mathrm{Ar}}\right), 70.7\left(\mathrm{CH}_{2}\right), 50.6(\mathrm{~S}-\mathrm{CH}), 42.6$ $\left(\mathrm{SO}_{2} \mathrm{CH}_{3}\right), 40.7\left(\mathrm{CH}_{2}\right), 34.3\left(\mathrm{CH}_{2}\right), 30.2\left(\mathrm{CH}_{3}\right), 29.6\left(\mathrm{CH}_{2}\right), 28.3\left(\mathrm{CH}_{2}\right), 13.9\left(\mathrm{CH}_{3}\right)$.

IR (neat), $v\left(\mathrm{~cm}^{-1}\right):$ 2929, 1709, 1562, 1454, 1353, 1215, 1150, 1108, 1043, 963, 884, 776. HRMS (ESI) m/z: $[\mathrm{M}+\mathrm{H}]^{+}$Calcd for $\mathrm{C}_{17} \mathrm{H}_{24} \mathrm{ClN}_{2} \mathrm{O}_{5} \mathrm{~S}_{3}$ 467.0530; Found 467.0535. $\mathrm{R}_{\mathrm{f}}=0.33(\mathrm{PE} / \mathrm{EtOAc}=40 \%)$. 


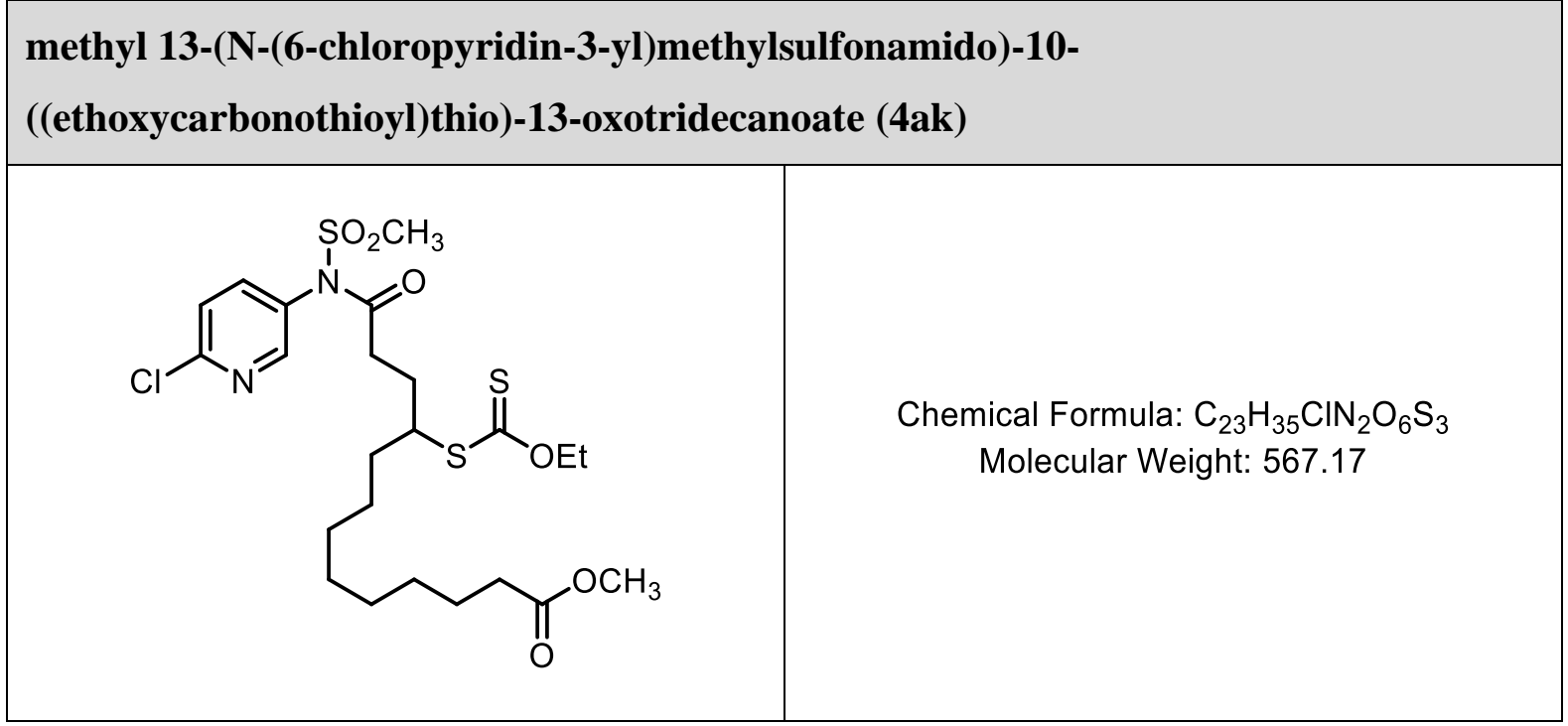

Following the general procedure $\mathrm{D}$, the reaction was carried out with the solution of 3a (300 $\mathrm{mg}, 0.81 \mathrm{mmol}, 1$ eq.) and methyl 10-undecenoate ( $0.37 \mathrm{~mL} 1.63 \mathrm{mmol}, 2$ eq. $)$ in EtOAc (0.82 $\mathrm{mL})$ with 0.2 eq. of DLP $(70 \mathrm{mg})$. Flash column chromatography on silica gel (eluent: $\mathrm{PE} / \mathrm{EtOAc}=0 \% \rightarrow 30 \%)$ afforded target product 4ak $(396 \mathrm{mg}, 86 \%)$ as a yellow oil.

${ }^{1} \mathrm{H}$ NMR (400 MHz, Chloroform- $d$ ) $\delta 8.29\left(\mathrm{dd}, J=2.8,0.7 \mathrm{~Hz}, 1 \mathrm{H}, \mathrm{CH}_{\mathrm{Ar}}\right), 7.59$ (dd, $J=8.4$, $\left.2.8 \mathrm{~Hz}, 1 \mathrm{H}, \mathrm{CH}_{\mathrm{Ar}}\right), 7.44\left(\mathrm{dd}, J=8.4,0.7 \mathrm{~Hz}, 1 \mathrm{H}, \mathrm{CH}_{\mathrm{Ar}}\right), 4.68-4.56\left(\mathrm{~m}, 2 \mathrm{H}, \mathrm{CH}_{2}\right), 3.76-3.68$ (m, 1H, S-CH), 3.65 (s, 3H, $\left.\mathrm{CH}_{3}\right), 3.49$ (s, 3H, SO $\left.\mathrm{SO}_{3}\right), 2.32-2.12\left(\mathrm{~m}, 5 \mathrm{H}, 2 \times \mathrm{CH}_{2}\right.$ and $\left.\mathrm{CH}_{A l} \mathrm{H}\right), 1.81-1.69\left(\mathrm{~m}, 1 \mathrm{H}, \mathrm{CH} H_{A 2}\right), 1.69-1.53\left(\mathrm{~m}, 4 \mathrm{H}, 2 \times \mathrm{CH}_{2}\right), 1.41(\mathrm{t}, J=7.1 \mathrm{~Hz}, 3 \mathrm{H}$, $\left.\mathrm{CH}_{3}\right), 1.38-1.19\left(\mathrm{~m}, 10 \mathrm{H}, 5 \times \mathrm{CH}_{2}\right)$.

${ }^{13} \mathrm{C}$ NMR (101 MHz, Chloroform- $\left.d\right) \delta 215.2(\mathrm{~S}=\mathrm{O}), 174.4(\mathrm{C}=\mathrm{O}), 172.7(\mathrm{C}=\mathrm{O}), 153.0\left(\mathrm{C}_{\mathrm{Ar}}\right)$, $150.3\left(\mathrm{CH}_{\mathrm{Ar}}\right), 140.2\left(\mathrm{CH}_{\mathrm{Ar}}\right), 131.4\left(\mathrm{C}_{\mathrm{Ar}}\right), 125.6\left(\mathrm{CH}_{\mathrm{Ar}}\right), 70.5\left(\mathrm{CH}_{2}\right), 51.6\left(\mathrm{CH}_{3}\right), 51.1(\mathrm{~S}-\mathrm{CH})$, $42.6\left(\mathrm{SO}_{2} \mathrm{CH}_{3}\right), 35.0\left(\mathrm{CH}_{2}\right), 34.4\left(\mathrm{CH}_{2}\right), 34.2\left(\mathrm{CH}_{2}\right), 29.3\left(\mathrm{CH}_{2}\right), 29.3\left(\mathrm{CH}_{2}\right), 29.3\left(\mathrm{CH}_{2}\right), 29.2$ $\left(\mathrm{CH}_{2}\right), 29.2\left(\mathrm{CH}_{2}\right), 26.9\left(\mathrm{CH}_{2}\right), 25.0\left(\mathrm{CH}_{2}\right), 13.9\left(\mathrm{CH}_{3}\right)$.

IR (neat), $v\left(\mathrm{~cm}^{-1}\right)$ : 2927, 2854, 1720, 1562, 1455, 1357, 1212, 1156, 1109, 1048, 965, 777.

HRMS (ESI) m/z: $[\mathrm{M}+\mathrm{H}]^{+}$Calcd for $\mathrm{C}_{23} \mathrm{H}_{36} \mathrm{ClN}_{2} \mathrm{O}_{6} \mathrm{~S}_{3}$ 567.1419; Found 567.1420.

$\mathrm{R}_{\mathrm{f}}=0.41(\mathrm{PE} / \mathrm{EtOAc}=30 \%)$. 


\section{S-(5-(N-(6-chloropyridin-3-yl)methylsulfonamido)-1-(3,9-dimethyl-2,6-dioxo-2,3,6,9- tetrahydro-1H-purin-1-yl)-5-oxopentan-2-yl) O-ethyl carbonodithioate (4al)}<smiles>CCOC(=S)SC(CCC(=O)N(c1ccc(Cl)nc1)S(C)(=O)=O)Cn1c(=O)c2ncn(C)c2n(C)c1=O</smiles>

Chemical Formula: $\mathrm{C}_{21} \mathrm{H}_{25} \mathrm{CIN}_{6} \mathrm{O}_{6} \mathrm{~S}_{3}$ Molecular Weight: 589.10

Following the general procedure D, the reaction was carried out with the solution of 3a (300 $\mathrm{mg}, 0.81 \mathrm{mmol}, 1.5$ eq.) and 1-allyl-3,9-dimethyl-3,9-dihydro-1H-purine-2,6-dione (120 mg, $0.54 \mathrm{mmol}, 1$ eq.) in EtOAc $(0.82 \mathrm{~mL})$ with 0.6 eq. of DLP $(132 \mathrm{mg})$. Flash column chromatography on silica gel (eluent: $\mathrm{PE} / \mathrm{EtOAc}=80 \% \rightarrow 100 \%$ ) afforded target product 4al (195 $\mathrm{mg}, 61 \%)$ as a yellow oil.

${ }^{1} \mathrm{H}$ NMR (400 MHz, Chloroform- $d$ ) $\delta 8.32\left(\mathrm{dd}, J=2.8,0.7 \mathrm{~Hz}, 1 \mathrm{H}, \mathrm{CH}_{\mathrm{Ar}}\right), 7.64(\mathrm{dd}, J=8.4$, $\left.2.8 \mathrm{~Hz}, 1 \mathrm{H}, \mathrm{CH}_{\mathrm{Ar}}\right), 7.50(\mathrm{q}, J=0.6 \mathrm{~Hz}, 1 \mathrm{H}, \mathrm{CH}), 7.45\left(\mathrm{dd}, J=8.4,0.7 \mathrm{~Hz}, 1 \mathrm{H}, \mathrm{CH}_{\mathrm{Ar}}\right), 4.56(\mathrm{q}$, $\left.J=7.1 \mathrm{~Hz}, 2 \mathrm{H}, \mathrm{CH}_{2}\right), 4.27\left(\mathrm{dd}, J=12.7,8.1 \mathrm{~Hz}, 1 \mathrm{H}, \mathrm{CH}_{A l} \mathrm{H}\right), 4.22-4.13$ (m, 1H, S-CH), 4.09 (dd, $\left.J=12.7,6.5 \mathrm{~Hz}, 1 \mathrm{H}, \mathrm{CH} H_{A 2}\right), 3.95\left(\mathrm{~d}, J=0.6 \mathrm{~Hz}, 3 \mathrm{H}, \mathrm{CH}_{3}\right), 3.54\left(\mathrm{~s}, 3 \mathrm{H}, \mathrm{CH}_{3}\right), 3.48$ (s, $\left.3 \mathrm{H}, \mathrm{SO}_{2} \mathrm{CH}_{3}\right), 2.46-2.25\left(\mathrm{~m}, 2 \mathrm{H}, \mathrm{CH}_{2}\right), 2.22-2.09\left(\mathrm{~m}, 1 \mathrm{H}, \mathrm{CH}_{B l} \mathrm{H}\right), 2.01-1.87(\mathrm{~m}, 1 \mathrm{H}$, $\left.\mathrm{CH} H_{B 2}\right), 1.37\left(\mathrm{t}, J=7.1 \mathrm{~Hz}, 3 \mathrm{H}, \mathrm{CH}_{3}\right)$.

${ }^{13} \mathrm{C}$ NMR (101 MHz, Chloroform- $\left.d\right) \delta 213.3(\mathrm{C}=\mathrm{S}), 172.4(\mathrm{C}=\mathrm{O}), 155.1(\mathrm{C}=\mathrm{O}), 153.0\left(\mathrm{C}_{\mathrm{Ar}}\right)$, $151.5(\mathrm{C}=\mathrm{O}), 150.3\left(\mathrm{CH}_{\mathrm{Ar}}\right), 149.1(\mathrm{C}), 141.9(\mathrm{CH}), 140.2\left(\mathrm{CH}_{\mathrm{Ar}}\right), 131.4\left(\mathrm{C}_{\mathrm{Ar}}\right), 125.6\left(\mathrm{CH}_{\mathrm{Ar}}\right)$, $107.5(\mathrm{C}), 70.6\left(\mathrm{CH}_{2}\right), 48.4(\mathrm{~S}-\mathrm{CH}), 43.1\left(\mathrm{CH}_{2}\right), 42.5\left(\mathrm{SO}_{2} \mathrm{CH}_{3}\right), 34.2\left(\mathrm{CH}_{2}\right), 33.7\left(\mathrm{CH}_{3}\right), 29.9$ $\left(\mathrm{CH}_{3}\right), 26.6\left(\mathrm{CH}_{2}\right), 13.8\left(\mathrm{CH}_{3}\right)$.

IR (neat), $v\left(\mathrm{~cm}^{-1}\right):$ 2930, 1705, 1658, 1548, 1455, 1355, 1225, 1155, 1111, 1047, 965, 736. HRMS (ESI) m/z: [M + Na] $]^{+}$Calcd for $\mathrm{C}_{21} \mathrm{H}_{25} \mathrm{ClN}_{6} \mathrm{NaO}_{6} \mathrm{~S}_{3} 611.0578$; Found 611.0584. $\mathrm{R}_{\mathrm{f}}=0.31(\mathrm{EtOAc})$ 
S-(5-(N-(6-chloropyridin-3-yl)methylsulfonamido)-5-oxo-1-(perfluorophenyl)pentan-

2-yl) O-ethyl carbonodithioate (4am)<smiles>CCOC(=S)SC(CCC(=O)N(c1ccc(Cl)nc1)c1c(F)c(F)c(F)c(F)c1F)Cc1cc(Cl)c(F)c(F)c1F</smiles>

Chemical Formula: $\mathrm{C}_{20} \mathrm{H}_{18} \mathrm{ClF}_{5} \mathrm{~N}_{2} \mathrm{O}_{4} \mathrm{~S}_{3}$ Molecular Weight: 577.00

Following the general procedure D, the reaction was carried out with the solution of 3a (300 $\mathrm{mg}, 0.81 \mathrm{mmol}, 1$ eq.) and allylpentafluorobenzene $(0.25 \mathrm{~mL}, 1.63 \mathrm{mmol}, 2$ eq. $)$ in EtOAc $(0.82 \mathrm{~mL})$ with 0.2 eq. of DLP (64 mg). Flash column chromatography on silica gel (eluent: $\mathrm{PE} / \mathrm{EtOAc}=0 \% \rightarrow 30 \%)$ afforded target product $\mathbf{4 a m}(331 \mathrm{mg}, 71 \%)$ as a yellow oil.

${ }^{1} \mathrm{H}$ NMR (400 MHz, Chloroform- $d$ ) $\delta 8.27$ (dd, $J=2.7,0.7 \mathrm{~Hz}, 1 \mathrm{H}, \mathrm{CH}_{\mathrm{Ar}}$ ), $7.58(\mathrm{dd}, J=8.4$, $\left.2.7 \mathrm{~Hz}, 1 \mathrm{H}, \mathrm{CH}_{\mathrm{Ar}}\right), 7.45\left(\mathrm{dd}, J=8.4,0.7 \mathrm{~Hz}, 1 \mathrm{H}, \mathrm{CH}_{\mathrm{Ar}}\right), 4.60\left(\mathrm{q}, J=7.1 \mathrm{~Hz}, 2 \mathrm{H}, \mathrm{CH}_{2}\right), 4.07-$ 3.95 (m, 1H, S-CH), 3.49 (s, 3H, $\left.\mathrm{SO}_{2} \mathrm{CH}_{3}\right), 3.14-2.97$ (m, 2H, $\left.\mathrm{CH}_{2}\right), 2.35-2.25$ (m, 2H, $\left.\mathrm{CH}_{2}\right), 2.25-2.13\left(\mathrm{~m}, 1 \mathrm{H}, \mathrm{CH}_{A l} \mathrm{H}\right), 1.88-1.77\left(\mathrm{~m}, 1 \mathrm{H}, \mathrm{CH} H_{A 2}\right), 1.41\left(\mathrm{t}, J=7.1 \mathrm{~Hz}, 3 \mathrm{H}, \mathrm{CH}_{3}\right)$. ${ }^{13} \mathrm{C}$ NMR (101 MHz, Chloroform- $\left.d\right) \delta 213.2(\mathrm{C}=\mathrm{S}), 172.2(\mathrm{C}=\mathrm{O}), 153.2\left(\mathrm{C}_{\mathrm{Ar}}\right), 150.2\left(\mathrm{CH}_{\mathrm{Ar}}\right)$, $140.1\left(\mathrm{CH}_{\mathrm{Ar}}\right), 131.3\left(\mathrm{C}_{\mathrm{Ar}}\right), 125.7\left(\mathrm{CH}_{\mathrm{Ar}}\right), 111.3\left(\mathrm{C}_{\mathrm{Ar}}\right), 71.0\left(\mathrm{CH}_{2}\right), 49.7(\mathrm{~S}-\mathrm{CH}), 42.6\left(\mathrm{SO}_{2} \mathrm{CH}_{3}\right)$, $34.3\left(\mathrm{CH}_{2}\right), 28.3\left(\mathrm{CH}_{2}\right), 28.2\left(\mathrm{CH}_{2}\right), 13.8\left(\mathrm{CH}_{3}\right)$. C-F signals are not observed.

IR (neat), $v\left(\mathrm{~cm}^{-1}\right):$ 2933, 1712, 1520, 1502, 1455, 1357, 1222, 1151, 1110, 1045, 963, 777 , 735 .

HRMS (ESI) m/z: $[\mathrm{M}+\mathrm{H}]^{+}$Calcd for $\mathrm{C}_{20} \mathrm{H}_{19} \mathrm{ClF}_{5} \mathrm{~N}_{2} \mathrm{O}_{4} \mathrm{~S}_{3}$ 577.0110; Found 577.0112.

$\mathrm{R}_{\mathrm{f}}=0.60(\mathrm{PE} / \mathrm{EtOAc}=40 \%)$. 


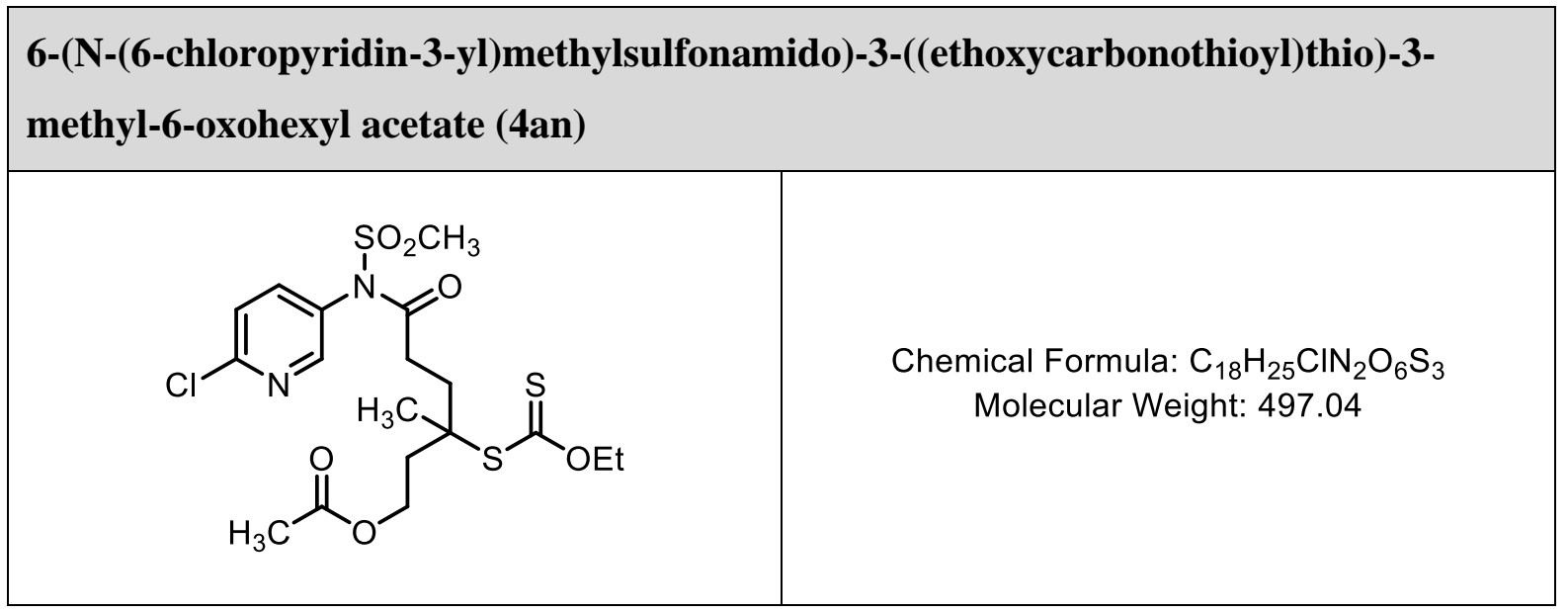

Following the general procedure D, the reaction was carried out with the solution of 3a (300 $\mathrm{mg}, 0.81 \mathrm{mmol}, 1$ eq.) and 3-methylbut-3-en-1-yl acetate (210 mg, $1.64 \mathrm{mmol}, 2$ eq.) in EtOAc $(0.82 \mathrm{~mL})$ with $0.3 \mathrm{eq}$. of DLP (96 mg). Flash column chromatography on silica gel (eluent: $\mathrm{PE} / \mathrm{EtOAc}=0 \% \rightarrow 20 \% \rightarrow 60 \%)$ afforded target product $4 \mathbf{a n}(294 \mathrm{mg}, 73 \%)$ as a yellow oil.

${ }^{1} \mathrm{H}$ NMR (400 MHz, Chloroform- $d$ ) $\delta 8.34\left(\mathrm{~d}, J=2.8 \mathrm{~Hz}, 1 \mathrm{H}, \mathrm{CH}_{\mathrm{Ar}}\right), 7.65(\mathrm{dd}, J=8.4,2.8 \mathrm{~Hz}$, $\left.1 \mathrm{H}, \mathrm{CH}_{\mathrm{Ar}}\right), 7.49\left(\mathrm{~d}, J=8.4 \mathrm{~Hz}, 1 \mathrm{H}, \mathrm{CH}_{\mathrm{Ar}}\right), 4.70-4.60\left(\mathrm{~m}, 2 \mathrm{H}, \mathrm{CH}_{2}\right), 4.15(\mathrm{t}, J=7.0 \mathrm{~Hz}, 2 \mathrm{H}$, $\left.\mathrm{CH}_{2}\right), 3.47\left(\mathrm{~s}, 3 \mathrm{H}, \mathrm{SO}_{2} \mathrm{CH}_{3}\right), 2.45-2.18\left(\mathrm{~m}, 4 \mathrm{H}, \mathrm{CH}_{2}, \mathrm{CH}_{A l} \mathrm{H}, \mathrm{CH}_{B l} \mathrm{H}\right), 2.08-2.00(\mathrm{~m}, 1 \mathrm{H}$, $\mathrm{CH}_{A 2}$ ), $2.02\left(\mathrm{~s}, 3 \mathrm{H}, \mathrm{CH}_{3}\right), 2.00-1.87\left(\mathrm{~m}, 1 \mathrm{H}, \mathrm{CH}_{B 2}\right), 1.47\left(\mathrm{t}, J=7.1 \mathrm{~Hz}, 3 \mathrm{H}, \mathrm{CH}_{3}\right), 1.23(\mathrm{~s}$, $\left.3 \mathrm{H}, \mathrm{CH}_{3}\right)$.

${ }^{13} \mathrm{C}$ NMR (101 MHz, Chloroform- $\left.d\right) \delta 211.7(\mathrm{C}=\mathrm{S}), 172.5(\mathrm{C}=\mathrm{O}), 171.0(\mathrm{C}=\mathrm{O}), 153.2\left(\mathrm{C}_{\mathrm{Ar}}\right)$, $150.1\left(\mathrm{CH}_{\mathrm{Ar}}\right), 140.1\left(\mathrm{CH}_{\mathrm{Ar}}\right), 131.4\left(\mathrm{C}_{\mathrm{Ar}}\right), 125.8\left(\mathrm{CH}_{\mathrm{Ar}}\right), 70.2\left(\mathrm{CH}_{2}\right), 60.9\left(\mathrm{CH}_{2}\right), 55.9(\mathrm{C}), 42.7$ $\left(\mathrm{SO}_{2} \mathrm{CH}_{3}\right), 38.1\left(\mathrm{CH}_{2}\right), 34.1\left(\mathrm{CH}_{2}\right), 32.3\left(\mathrm{CH}_{2}\right), 24.8\left(\mathrm{CH}_{3}\right), 21.1\left(\mathrm{CH}_{3}\right), 13.8\left(\mathrm{CH}_{3}\right)$.

IR (neat), $v\left(\mathrm{~cm}^{-1}\right):$ 2932, 1721, 1456, 1358, 1233, 1156, 1110, 1037, 965, 777, 735. HRMS (ESI) m/z: $[\mathrm{M}+\mathrm{H}]^{+}$Calcd for $\mathrm{C}_{18} \mathrm{H}_{26} \mathrm{ClN}_{2} \mathrm{O}_{6} \mathrm{~S}_{3}$ 497.0636; Found 497.0630. $\mathrm{R}_{\mathrm{f}}=0.26(\mathrm{PE} / \mathrm{EtOAc}=40 \%)$. 


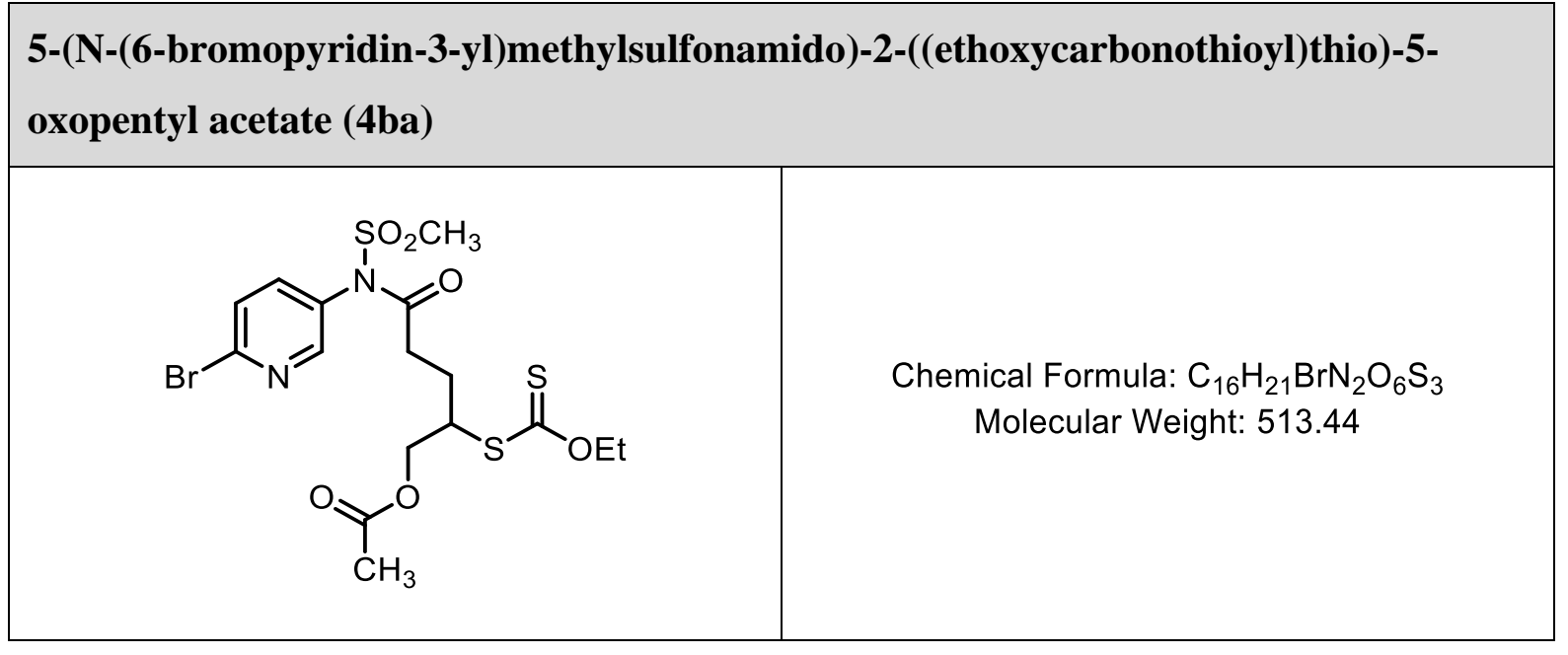

Following the general procedure $\mathrm{D}$, the reaction was carried out with the solution of $\mathbf{3 b}$ (300 $\mathrm{mg}, 0.73 \mathrm{mmol}, 1$ eq.) and allyl acetate $(0.16 \mathrm{~mL}, 1.45 \mathrm{mmol}, 2$ eq. $)$ in EtOAc $(0.73 \mathrm{~mL})$ with 0.2 eq. of DLP (60 mg). Flash column chromatography on silica gel (eluent: PE/EtOAc $=0 \%$ $\rightarrow 40 \%)$ afforded target product $\mathbf{4 b a}(292 \mathrm{mg}, 78 \%)$ as a colorless oil.

${ }^{1} \mathrm{H}$ NMR (400 MHz, Chloroform- $d$ ) $\delta 8.28\left(\mathrm{dd}, J=2.8,0.7 \mathrm{~Hz}, 1 \mathrm{H}, \mathrm{CH}_{\mathrm{Ar}}\right), 7.62(\mathrm{dd}, J=8.4$, $\left.0.7 \mathrm{~Hz}, 1 \mathrm{H}, \mathrm{CH}_{\mathrm{Ar}}\right), 7.49\left(\mathrm{dd}, J=8.4,2.8 \mathrm{~Hz}, 1 \mathrm{H}, \mathrm{CH}_{\mathrm{Ar}}\right), 4.64\left(\mathrm{q}, J=7.1 \mathrm{~Hz}, 2 \mathrm{H}, \mathrm{CH}_{2}\right), 4.27$ $\left(\mathrm{dd}, J=11.5,4.9 \mathrm{~Hz}, 1 \mathrm{H}, \mathrm{CH} H_{A l} \mathrm{H}\right), 4.18\left(\mathrm{dd}, J=11.5,6.1 \mathrm{~Hz}, 1 \mathrm{H}, \mathrm{CH} H_{A 2}\right), 4.02-3.93(\mathrm{~m}, 1 \mathrm{H}$, $\mathrm{S}-\mathrm{CH}), 3.49$ (s, 3H, $\left.\mathrm{SO}_{2} \mathrm{CH}_{3}\right), 2.33-2.25\left(\mathrm{~m}, 2 \mathrm{H}, \mathrm{CH}_{2}\right), 2.26-2.15\left(\mathrm{~m}, 1 \mathrm{H}, \mathrm{CH}_{B 1} \mathrm{H}\right), 2.05$ (s, $\left.3 \mathrm{H}, \mathrm{CH}_{3}\right), 1.92-1.79\left(\mathrm{~m}, 1 \mathrm{H}, \mathrm{CH} H_{B 2}\right), 1.42\left(\mathrm{t}, J=7.1 \mathrm{~Hz}, 3 \mathrm{H}, \mathrm{CH}_{3}\right)$.

${ }^{13} \mathrm{C}$ NMR (101 MHz, Chloroform- $\left.d\right) \delta 213.2(\mathrm{C}=\mathrm{S}), 172.2(\mathrm{C}=\mathrm{O}), 170.7(\mathrm{C}=\mathrm{O}), 150.6\left(\mathrm{CH}_{\mathrm{Ar}}\right)$, $143.8\left(\mathrm{C}_{\mathrm{Ar}}\right), 139.9\left(\mathrm{CH}_{\mathrm{Ar}}\right), 131.8\left(\mathrm{C}_{\mathrm{Ar}}\right), 129.5\left(\mathrm{CH}_{\mathrm{Ar}}\right), 71.0\left(\mathrm{CH}_{2}\right), 65.8\left(\mathrm{CH}_{2}\right), 48.9(\mathrm{~S}-\mathrm{CH})$, $42.6\left(\mathrm{SO}_{2} \mathrm{CH}_{3}\right), 34.2\left(\mathrm{CH}_{2}\right), 25.8\left(\mathrm{CH}_{2}\right), 20.9\left(\mathrm{CH}_{3}\right), 13.9\left(\mathrm{CH}_{3}\right)$.

IR (neat), $v\left(\mathrm{~cm}^{-1}\right): 1733,1712,1451,1355,1222,1151,1089,1039,964,884,771,745,547$. HRMS (ESI) m/z: $[\mathrm{M}+\mathrm{H}]^{+}$Calcd for $\mathrm{C}_{16} \mathrm{H}_{22} \mathrm{BrN}_{2} \mathrm{O}_{6} \mathrm{~S}_{3}$ 512.9818; Found 512.9819. $\mathrm{R}_{\mathrm{f}}=0.37(\mathrm{PE} / \mathrm{EtOAc}=40 \%)$. 


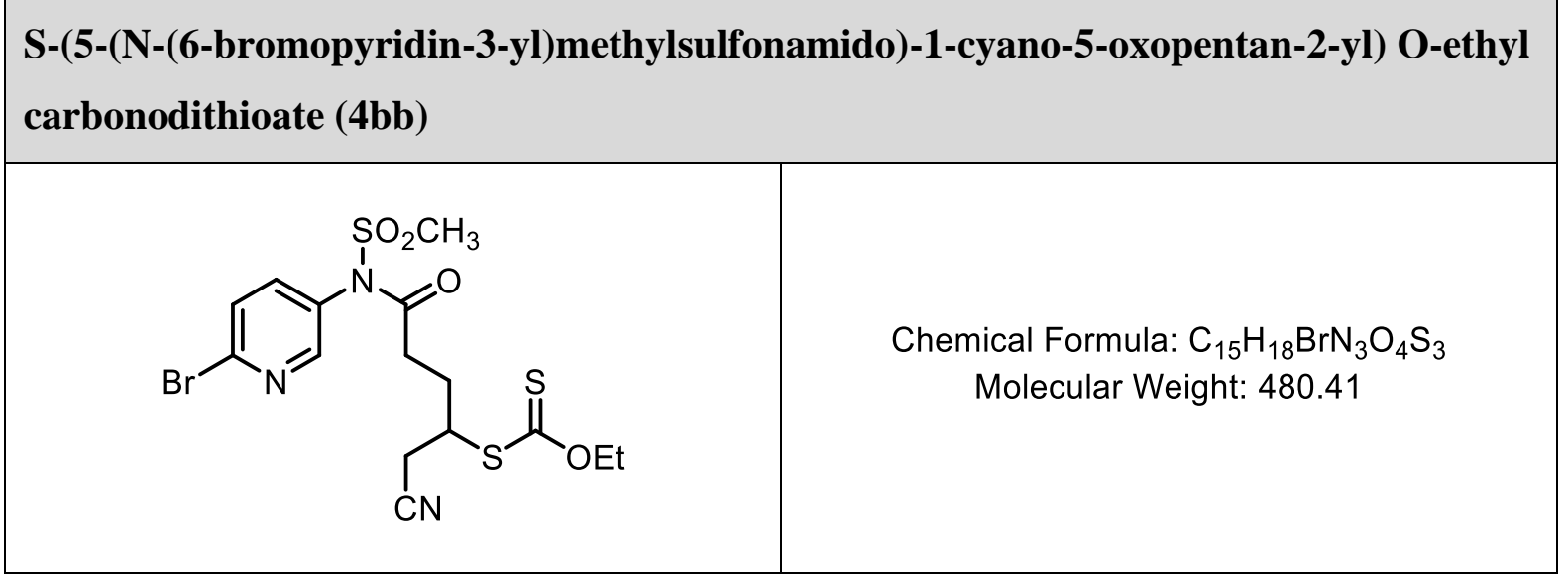

Following the general procedure D, the reaction was carried out with the solution of $\mathbf{3 b}$ (300 $\mathrm{mg}, 0.73 \mathrm{mmol}, 1$ eq.) and allyl cyanide $(0.12 \mathrm{~mL}, 1.45 \mathrm{mmol}, 2$ eq. $)$ in EtOAc $(0.73 \mathrm{~mL})$ with 0.3 eq. of DLP $(90 \mathrm{mg})$. Flash column chromatography on silica gel (eluent: PE/EtOAc $=0 \%$ $\rightarrow 50 \%)$ afforded target product $\mathbf{4 b b}(260 \mathrm{mg}, 75 \%)$ as a colorless oil.

${ }^{1} \mathrm{H}$ NMR (400 MHz, Chloroform- $d$ ) $\delta 8.28\left(\mathrm{dd}, J=2.8,0.7 \mathrm{~Hz}, 1 \mathrm{H}, \mathrm{CH}_{\mathrm{Ar}}\right), 7.62(\mathrm{dd}, J=8.4$, $\left.0.7 \mathrm{~Hz}, 1 \mathrm{H}, \mathrm{CH}_{\mathrm{Ar}}\right), 7.52\left(\mathrm{dd}, J=8.4,2.8 \mathrm{~Hz}, 1 \mathrm{H}, \mathrm{CH}_{\mathrm{Ar}}\right), 4.63\left(\mathrm{~d}, J=7.1 \mathrm{~Hz}, 2 \mathrm{H}, \mathrm{CH}_{2}\right), 3.95-$ 3.86 (m, 1H, S-CH), 3.47 (s, 3H, SO $\left.\mathrm{CH}_{3}\right), 2.91-2.81$ (m, 2H, $\left.\mathrm{CH}_{2}\right), 2.37-2.29$ (m, 2H, $\left.\mathrm{CH}_{2}\right), 2.28-2.19\left(\mathrm{~m}, 1 \mathrm{H}, \mathrm{CH}_{A I} \mathrm{H}\right), 2.05-1.91\left(\mathrm{~m}, 1 \mathrm{H}, \mathrm{CH} H_{A 2}\right), 1.42\left(\mathrm{t}, J=7.1 \mathrm{~Hz}, 3 \mathrm{H}, \mathrm{CH}_{3}\right)$. ${ }^{13} \mathrm{C}$ NMR (101 MHz, Chloroform- $\left.d\right) \delta 211.9(\mathrm{C}=\mathrm{S}), 171.8(\mathrm{C}=\mathrm{O}), 150.6\left(\mathrm{CH}_{\mathrm{Ar}}\right), 143.7\left(\mathrm{C}_{\mathrm{Ar}}\right)$, $139.9\left(\mathrm{CH}_{\mathrm{Ar}}\right), 131.6\left(\mathrm{C}_{\mathrm{Ar}}\right), 129.5\left(\mathrm{CH}_{\mathrm{Ar}}\right), 117.0(\mathrm{CN}), 71.2\left(\mathrm{CH}_{2}\right), 45.7(\mathrm{CH}), 42.5\left(\mathrm{SO}_{2} \mathrm{CH}_{3}\right)$, $34.1\left(\mathrm{CH}_{2}\right), 27.3\left(\mathrm{CH}_{2}\right), 24.3\left(\mathrm{CH}_{2}\right), 13.8\left(\mathrm{CH}_{3}\right)$.

IR (neat), $v\left(\mathrm{~cm}^{-1}\right): 1708,1450,1353,1224,1149,1089,1043,962,772,734,546$.

HRMS (ESI) m/z: $[\mathrm{M}+\mathrm{H}]^{+}$Calcd for $\mathrm{C}_{15} \mathrm{H}_{19} \mathrm{BrN}_{3} \mathrm{O}_{4} \mathrm{~S}_{3} 479.9716$; Found 479.9717. $\mathrm{R}_{\mathrm{f}}=0.31(\mathrm{PE} / \mathrm{EtOAc}=40 \%)$. 


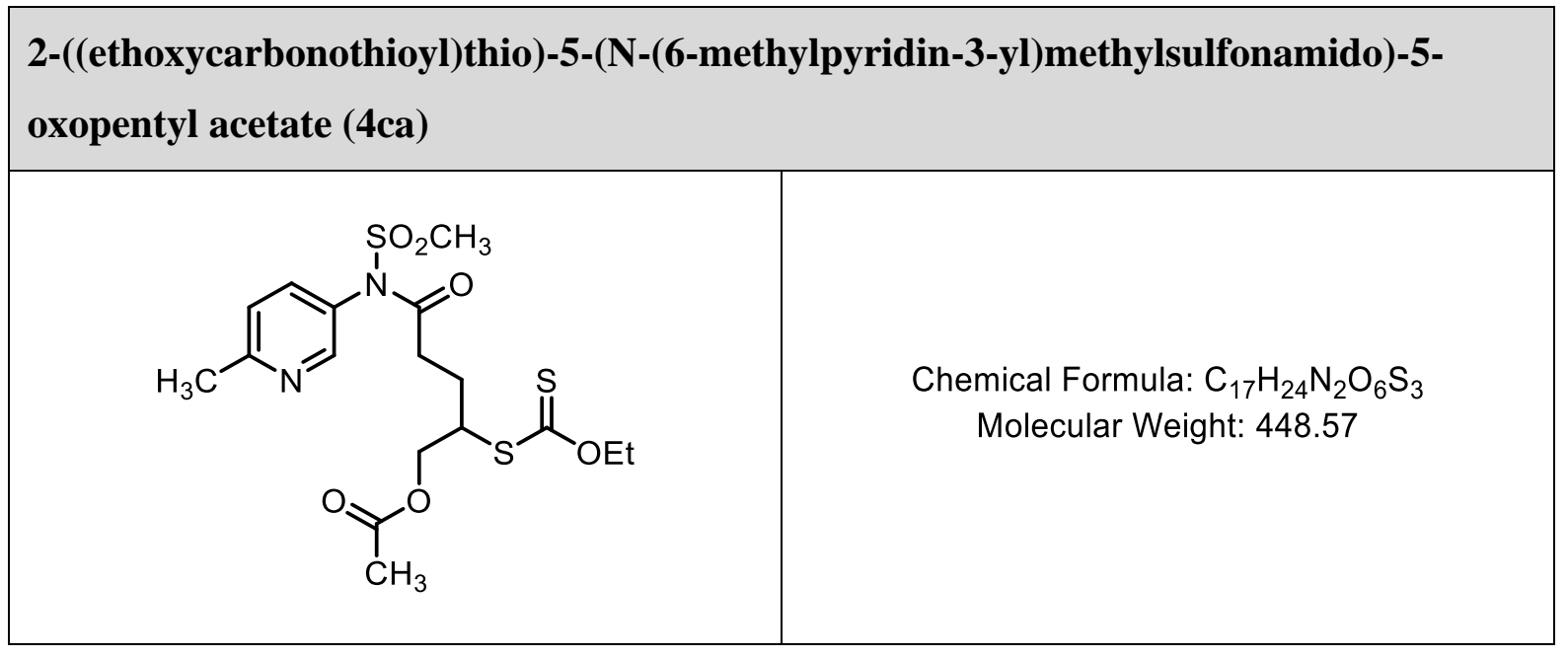

Following the general procedure D, the reaction was carried out with the solution of $\mathbf{3 c}$ (300 $\mathrm{mg}, 0.86 \mathrm{mmol}, 1$ eq.) and allyl acetate $(0.19 \mathrm{~mL}, 1.72 \mathrm{mmol}, 2$ eq. $)$ in EtOAc $(0.86 \mathrm{~mL})$ with 0.2 eq. of DLP $(70 \mathrm{mg})$. Flash column chromatography on silica gel (eluent: PE/EtOAc $=0 \%$ $\rightarrow 20 \% \rightarrow 60 \%$ ) afforded target product $\mathbf{4 c a}(341 \mathrm{mg}, 88 \%)$ as a colorless oil.

${ }^{1} \mathrm{H}$ NMR (400 MHz, Chloroform- $d$ ) $\delta 8.37\left(\mathrm{dd}, J=2.7,0.7 \mathrm{~Hz}, 1 \mathrm{H}, \mathrm{CH}_{\mathrm{Ar}}\right), 7.50(\mathrm{dd}, J=8.1$, $\left.2.7 \mathrm{~Hz}, 1 \mathrm{H}, \mathrm{CH}_{\mathrm{Ar}}\right), 7.28\left(\mathrm{dd}, J=8.1,0.7 \mathrm{~Hz}, 1 \mathrm{H}, \mathrm{CH}_{\mathrm{Ar}}\right), 4.62\left(\mathrm{q}, J=7.1 \mathrm{~Hz}, 2 \mathrm{H}, \mathrm{CH}_{2}\right), 4.24$ $\left(\mathrm{dd}, J=11.4,4.8 \mathrm{~Hz}, 1 \mathrm{H}, \mathrm{CH} H_{A l} \mathrm{H}\right), 4.16\left(\mathrm{dd}, J=11.4,6.1 \mathrm{~Hz}, 1 \mathrm{H}, \mathrm{CH} H_{A 2}\right), 3.99-3.90(\mathrm{~m}, 1 \mathrm{H}$, $\mathrm{S}-\mathrm{CH}), 3.47\left(\mathrm{~s}, 3 \mathrm{H}, \mathrm{SO}_{2} \mathrm{CH}_{3}\right), 2.62\left(\mathrm{~s}, 3 \mathrm{H}, \mathrm{CH}_{3}\right), 2.31-2.24\left(\mathrm{~m}, 2 \mathrm{H}, \mathrm{CH}_{2}\right), 2.24-2.13(\mathrm{~m}, 1 \mathrm{H}$, $\left.\mathrm{CH}_{B 1} \mathrm{H}\right), 2.03\left(\mathrm{~s}, 3 \mathrm{H}, \mathrm{CH}_{3}\right), 1.90-1.79\left(\mathrm{~m}, 1 \mathrm{H}, \mathrm{CH} H_{B 2}\right), 1.40\left(\mathrm{t}, J=7.1 \mathrm{~Hz}, 3 \mathrm{H}, \mathrm{CH}_{3}\right)$.

${ }^{13} \mathrm{C}$ NMR (101 MHz, Chloroform- $\left.d\right) \delta 213.0(\mathrm{C}=\mathrm{S}), 172.7(\mathrm{C}=\mathrm{O}), 170.7(\mathrm{C}=\mathrm{O}), 160.9\left(\mathrm{C}_{\mathrm{Ar}}\right)$, $149.5\left(\mathrm{CH}_{\mathrm{Ar}}\right), 137.6\left(\mathrm{CH}_{\mathrm{Ar}}\right), 129.7\left(\mathrm{C}_{\mathrm{Ar}}\right), 124.4\left(\mathrm{CH}_{\mathrm{Ar}}\right), 70.8\left(\mathrm{CH}_{2}\right), 65.7\left(\mathrm{CH}_{2}\right), 48.8(\mathrm{~S}-\mathrm{CH})$, $42.4\left(\mathrm{CH}_{3}\right), 34.1\left(\mathrm{CH}_{2}\right), 25.7\left(\mathrm{CH}_{2}\right), 24.5\left(\mathrm{CH}_{3}\right), 20.8\left(\mathrm{CH}_{3}\right), 13.8\left(\mathrm{CH}_{3}\right)$.

IR (neat), $v\left(\mathrm{~cm}^{-1}\right): 1737,1707,1486,1352,1222,1151,1039,964,762,734,546$.

HRMS (ESI) m/z: [M + H $]^{+}$Calcd for $\mathrm{C}_{17} \mathrm{H}_{25} \mathrm{~N}_{2} \mathrm{O}_{6} \mathrm{~S}_{3} 449.0869$; Found 449.0870.

$\mathrm{R}_{\mathrm{f}}=0.32(\mathrm{PE} / \mathrm{EtOAc}=50 \%)$. 


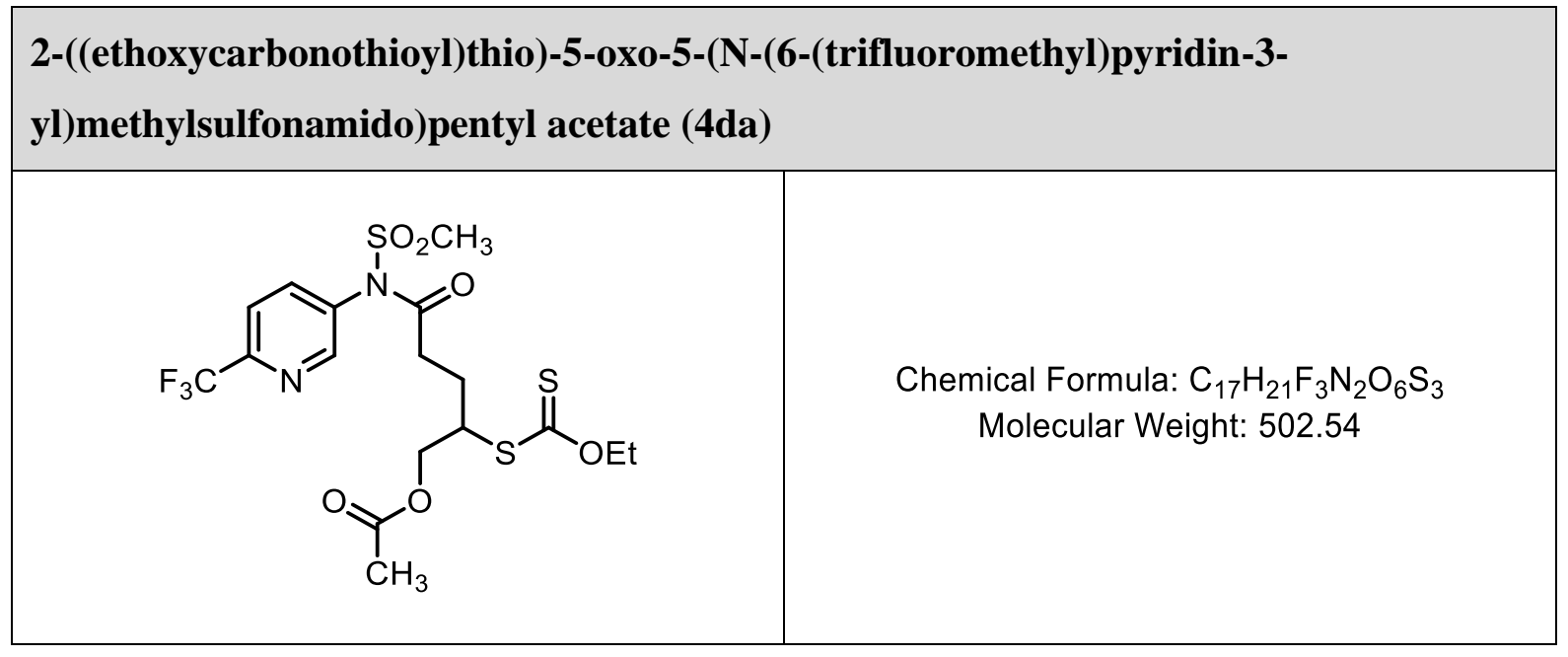

Following the general procedure D, the reaction was carried out with the solution of $\mathbf{3 d}$ (286 $\mathrm{mg}, 0.71 \mathrm{mmol}, 1$ eq.) and allyl acetate $(0.16 \mathrm{~mL}, 1.42 \mathrm{mmol}, 2$ eq. $)$ in EtOAc $(0.71 \mathrm{~mL})$ with 0.2 eq. of DLP (60 mg). Flash column chromatography on silica gel (eluent: PE/EtOAc $=0 \%$ $\rightarrow 40 \%)$ afforded target product $\mathbf{4 d a}(211 \mathrm{mg}, 59 \%)$ as a colorless oil.

${ }^{1} \mathrm{H}$ NMR (400 MHz, Chloroform- $d$ ) $\delta 8.62\left(\mathrm{dd}, J=2.3,0.9 \mathrm{~Hz}, 1 \mathrm{H}, \mathrm{CH}_{\mathrm{Ar}}\right), 7.86(\mathrm{ddd}, J=8.3$, $\left.2.3 \mathrm{~Hz}, 1 \mathrm{H}, \mathrm{CH}_{\mathrm{Ar}}\right), 7.82\left(\mathrm{dd}, J=8.3,0.9 \mathrm{~Hz}, 1 \mathrm{H}, \mathrm{CH}_{\mathrm{Ar}}\right), 4.61\left(\mathrm{q}, J=7.1 \mathrm{~Hz}, 2 \mathrm{H}, \mathrm{CH}_{2}\right), 4.25$ $\left(\mathrm{dd}, J=11.5,4.9 \mathrm{~Hz}, 1 \mathrm{H}, \mathrm{CH} H_{A l} \mathrm{H}\right), 4.17\left(\mathrm{dd}, J=11.5,6.0 \mathrm{~Hz}, 1 \mathrm{H}, \mathrm{CH} H_{A 2}\right), 4.00-3.92(\mathrm{~m}, 1 \mathrm{H}$, $\mathrm{S}-\mathrm{CH}), 3.50\left(\mathrm{~s}, 3 \mathrm{H}, \mathrm{SO}_{2} \mathrm{CH}_{3}\right), 2.33-2.26\left(\mathrm{~m}, 2 \mathrm{H}, \mathrm{CH}_{2}\right), 2.26-2.16\left(\mathrm{~m}, 1 \mathrm{H}, \mathrm{CH}_{B 1} \mathrm{H}\right), 2.03(\mathrm{~s}$, $\left.3 \mathrm{H}, \mathrm{CH}_{3}\right), 1.92-1.78\left(\mathrm{~m}, 1 \mathrm{H}, \mathrm{CH} H_{B 2}\right), 1.40\left(\mathrm{t}, J=7.1 \mathrm{~Hz}, 3 \mathrm{H}, \mathrm{CH}_{3}\right)$.

${ }^{13} \mathrm{C}$ NMR (101 MHz, Chloroform- $\left.d\right) \delta 213.2(\mathrm{C}=\mathrm{S}), 172.0(\mathrm{C}=\mathrm{O}), 170.7(\mathrm{C}=\mathrm{O}), 150.6\left(\mathrm{CH}_{\mathrm{Ar}}\right)$, $149.2\left(\mathrm{C}-\mathrm{F},{ }^{2} J_{C-F}=35.6 \mathrm{~Hz}, \mathrm{C}_{\mathrm{Ar}}\right), 139.3\left(\mathrm{CH}_{\mathrm{Ar}}\right), 134.7\left(\mathrm{C}_{\mathrm{Ar}}\right), 121.7\left(\mathrm{C}-\mathrm{F},{ }^{3} J_{C-F}=2.7 \mathrm{~Hz}, \mathrm{CH}_{\mathrm{Ar}}\right)$, $121.0\left(\mathrm{C}-\mathrm{F},{ }^{1} J_{C-F}=274.7 \mathrm{~Hz}, \mathrm{CF}_{3}\right), 71.0\left(\mathrm{CH}_{2}\right), 65.7\left(\mathrm{CH}_{2}\right), 48.9(\mathrm{~S}-\mathrm{CH}), 42.6\left(\mathrm{SO}_{2} \mathrm{CH}_{3}\right), 34.3$ $\left(\mathrm{CH}_{2}\right), 25.8\left(\mathrm{CH}_{2}\right), 20.8\left(\mathrm{CH}_{3}\right), 13.8\left(\mathrm{CH}_{3}\right)$.

IR (neat), $v\left(\mathrm{~cm}^{-1}\right): 1731,1714,1335,1226,1143,1041,966,777,761,602,540$.

HRMS (ESI) m/z: $[\mathrm{M}+\mathrm{H}]^{+}$Calcd for $\mathrm{C}_{17} \mathrm{H}_{22} \mathrm{~F}_{3} \mathrm{~N}_{2} \mathrm{O}_{6} \mathrm{~S}_{3}$ 503.0587; Found 503.0588.

$\mathrm{R}_{\mathrm{f}}=0.44(\mathrm{PE} / \mathrm{EtOAc}=40 \%)$. 
S-(1-cyano-5-oxo-5-(N-(6-(trifluoromethyl)pyridin-3-yl)methylsulfonamido)pentan-2yl) O-ethyl carbonodithioate $(4 \mathrm{db})$

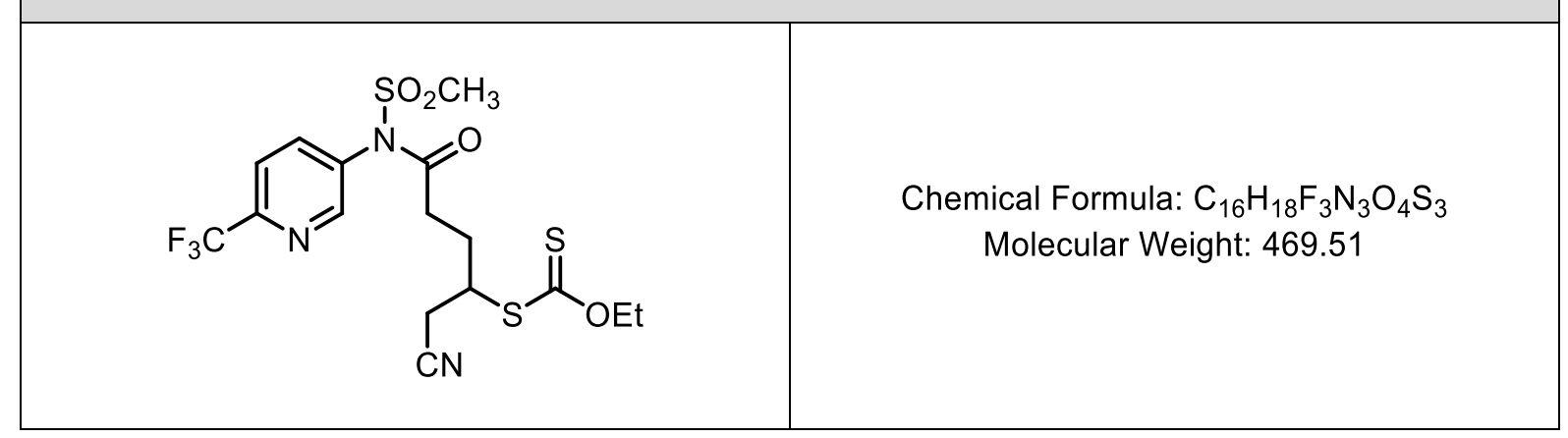

Following the general procedure D, the reaction was carried out with the solution of $\mathbf{3 d}$ (306 $\mathrm{mg}, 0.76 \mathrm{mmol}, 1$ eq.) and allyl cyanide $(0.12 \mathrm{~mL}, 1.49 \mathrm{mmol}, 2$ eq. $)$ in EtOAc $(0.75 \mathrm{~mL})$ with 0.2 eq. of DLP $(60 \mathrm{mg})$. Flash column chromatography on silica gel (eluent: PE/EtOAc $=0 \%$ $\rightarrow 40 \%)$ afforded target product $\mathbf{4 d b}(267 \mathrm{mg}, 75 \%)$ as a colorless oil.

${ }^{1} \mathrm{H}$ NMR (400 MHz, Chloroform- $d$ ) $\delta 8.64\left(\mathrm{dd}, J=2.3,1.0 \mathrm{~Hz}, 1 \mathrm{H}, \mathrm{CH}_{\mathrm{Ar}}\right), 7.88(\mathrm{dd}, J=8.3$, $\left.2.3 \mathrm{~Hz}, 1 \mathrm{H}, \mathrm{CH}_{\mathrm{Ar}}\right), 7.84\left(\mathrm{dd}, J=8.3,1.0 \mathrm{~Hz}, 1 \mathrm{H}, \mathrm{CH}_{\mathrm{Ar}}\right), 4.66\left(\mathrm{q}, J=7.1 \mathrm{~Hz}, 2 \mathrm{H}, \mathrm{CH}_{2}\right), 4.01-$ $3.92(\mathrm{~m}, 1 \mathrm{H}, \mathrm{S}-\mathrm{CH}), 3.52\left(\mathrm{~s}, 3 \mathrm{H}, \mathrm{SO}_{2} \mathrm{CH}_{3}\right), 2.92-2.82\left(\mathrm{~m}, 2 \mathrm{H}, \mathrm{CH}_{2}\right), 2.40-2.33(\mathrm{~m}, 2 \mathrm{H}$, $\left.\mathrm{CH}_{2}\right), 2.33-2.23\left(\mathrm{~m}, 1 \mathrm{H}, \mathrm{CH}_{A I} \mathrm{H}\right), 2.08-1.98\left(\mathrm{~m}, 1 \mathrm{H}, \mathrm{CH} H_{A 2}\right), 1.44\left(\mathrm{t}, J=7.1 \mathrm{~Hz}, 3 \mathrm{H}, \mathrm{CH}_{3}\right)$. ${ }^{13} \mathrm{C}$ NMR (101 MHz, Chloroform- $\left.d\right) \delta 212.0(\mathrm{C}=\mathrm{S}), 171.7(\mathrm{C}=\mathrm{O}), 150.6\left(\mathrm{CH}_{\mathrm{Ar}}\right), 149.5(\mathrm{C}-\mathrm{F}$, $\left.{ }^{2} J_{C-F}=35.9 \mathrm{~Hz}, \mathrm{C}_{\mathrm{Ar}}\right), 139.3\left(\mathrm{CH}_{\mathrm{Ar}}\right), 134.6\left(\mathrm{C}_{\mathrm{Ar}}\right), 121.9\left(\mathrm{C}-\mathrm{F},{ }^{3} J_{C-F}=2.5 \mathrm{~Hz}, \mathrm{CH}_{\mathrm{Ar}}\right), 121.0(\mathrm{C}-$ $\left.\mathrm{F},{ }^{1} J_{C-F}=274.9 \mathrm{~Hz}, \mathrm{CF}_{3}\right), 117.0(\mathrm{CN}), 71.3\left(\mathrm{CH}_{2}\right), 45.9(\mathrm{~S}-\mathrm{CH}), 42.8\left(\mathrm{SO}_{2} \mathrm{CH}_{3}\right), 34.3\left(\mathrm{CH}_{2}\right)$, $27.5\left(\mathrm{CH}_{2}\right), 24.5\left(\mathrm{CH}_{2}\right), 13.9\left(\mathrm{CH}_{3}\right)$.

IR (neat), $v\left(\mathrm{~cm}^{-1}\right): 2346,1714,1358,1336,1235,1151,1088,1047,966,759$.

HRMS (ESI) m/z: [M + H] $]^{+}$Calcd for $\mathrm{C}_{16} \mathrm{H}_{19} \mathrm{~F}_{3} \mathrm{~N}_{3} \mathrm{O}_{4} \mathrm{~S}_{3} 470.0484$; Found 470.0486.

$\mathrm{R}_{\mathrm{f}}=0.48(\mathrm{PE} / \mathrm{EtOAc}=40 \%)$. 
<smiles>CCOC(=S)SC(CC#N)CC(C)C(=O)N(c1ccc(Cl)nc1)S(C)(=O)=O</smiles>

Chemical Formula: $\mathrm{C}_{16} \mathrm{H}_{20} \mathrm{ClN}_{3} \mathrm{O}_{4} \mathrm{~S}_{3}$ Molecular Weight: 449.98

Following the general procedure $\mathrm{D}$, the reaction was carried out with the solution of $\mathbf{3 e}$ (300 $\mathrm{mg}, 0.78 \mathrm{mmol}, 1$ eq.) and allyl cyanide $(0.13 \mathrm{~mL}, 1.57 \mathrm{mmol}, 2$ eq. $)$ in EtOAc (0.78 mL) with 0.7 eq. of DLP (210 mg). Flash column chromatography on silica gel (eluent: PE/EtOAc $=0 \%$ $\rightarrow 40 \%)$ afforded target product $4 \mathrm{eb}(178 \mathrm{mg}, 50 \%)$ as a white foam. Mixture of two diastereomers (d.r. $=1.2: 1)$. Product contained traces of the reduced starting material $(\mathrm{N}-(6-$ chloropyridin-3-yl)- $N$-(methylsulfonyl)propionamide), inseparable by column chromatography.

Isomer 1:

${ }^{1} \mathrm{H}$ NMR (400 MHz, Chloroform- $d$ ) $\delta 8.26\left(\mathrm{dd}, J=2.8,0.7 \mathrm{~Hz}, 1 \mathrm{H}, \mathrm{CH}_{\mathrm{Ar}}\right), 7.59$ (dd, $J=8.4$, $\left.2.8 \mathrm{~Hz}, 1 \mathrm{H}, \mathrm{CH}_{\mathrm{Ar}}\right), 7.44\left(\mathrm{dd}, J=8.5,0.7 \mathrm{~Hz}, 1 \mathrm{H}, \mathrm{CH}_{\mathrm{Ar}}\right), 4.83-4.70\left(\mathrm{~m}, 2 \mathrm{H}, \mathrm{CH}_{2}\right), 3.98-3.87$ (m, 1H, S-CH), $3.46\left(\mathrm{~s}, 3 \mathrm{H}, \mathrm{SO}_{2} \mathrm{CH}_{3}\right), 2.91\left(\mathrm{dd}, J=17.1,5.6 \mathrm{~Hz}, 1 \mathrm{H}, \mathrm{CH} H_{A l} \mathrm{H}\right), 2.86-2.76(\mathrm{~m}$, $\left.1 \mathrm{H}, \mathrm{CH}_{A 2}\right), 2.63-2.52(\mathrm{~m}, 1 \mathrm{H}, \mathrm{CH}), 2.39-2.19\left(\mathrm{~m}, 1 \mathrm{H}, \mathrm{CH}_{B 1} \mathrm{H}\right), 1.81-1.69(\mathrm{~m}, 1 \mathrm{H}$, $\left.\mathrm{CH}_{B 2}\right), 1.50\left(\mathrm{t}, J=7.1 \mathrm{~Hz}, 3 \mathrm{H}, \mathrm{CH}_{3}\right), 1.14\left(\mathrm{~d}, J=6.9 \mathrm{~Hz}, 3 \mathrm{H}, \mathrm{CH}_{3}\right)$.

${ }^{13} \mathrm{C}$ NMR (101 MHz, Chloroform- $\left.d\right) \delta 211.1(\mathrm{C}=\mathrm{S}), 175.8(\mathrm{C}=\mathrm{O}), 153.1\left(\mathrm{C}_{\mathrm{Ar}}\right), 149.9\left(\mathrm{CH}_{\mathrm{Ar}}\right)$, $140.1\left(\mathrm{CH}_{\mathrm{Ar}}\right), 130.9\left(\mathrm{C}_{\mathrm{Ar}}\right), 125.4\left(\mathrm{CH}_{\mathrm{Ar}}\right), 116.7(\mathrm{CN}), 71.5\left(\mathrm{CH}_{2}\right), 45.2(\mathrm{~S}-\mathrm{CH}), 42.4\left(\mathrm{SO}_{2} \mathrm{CH}_{3}\right)$, $37.6(\mathrm{CH}), 36.0\left(\mathrm{CH}_{2}\right), 25.1\left(\mathrm{CH}_{2}\right), 18.4\left(\mathrm{CH}_{3}\right), 13.9\left(\mathrm{CH}_{3}\right)$.

Isomer 2:

${ }^{1} \mathrm{H}$ NMR (400 MHz, Chloroform- $d$ ) $\delta 8.31\left(\mathrm{dd}, J=2.8,0.7 \mathrm{~Hz}, 1 \mathrm{H}, \mathrm{CH}_{\mathrm{Ar}}\right), 7.64(\mathrm{dd}, J=8.4$, $\left.2.8 \mathrm{~Hz}, 1 \mathrm{H}, \mathrm{CH}_{\mathrm{Ar}}\right), 7.48\left(\mathrm{dd}, J=8.4,0.7 \mathrm{~Hz}, 1 \mathrm{H}, \mathrm{CH}_{\mathrm{Ar}}\right), 4.70-4.62\left(\mathrm{~m}, 2 \mathrm{H}, \mathrm{CH}_{2}\right), 3.82-3.72$ (m, 1H, S-CH), 3.49 (s, 3H, $\left.\mathrm{SO}_{2} \mathrm{CH}_{3}\right), 2.86-2.76\left(\mathrm{~m}, 2 \mathrm{H}, \mathrm{CH}_{2}\right), 2.50-2.40$ (m, 1H, CH), 2.39 $-2.19\left(\mathrm{~m}, 1 \mathrm{H}, \mathrm{CH}_{B 1} \mathrm{H}\right), 1.97-1.86\left(\mathrm{~m}, 1 \mathrm{H}, \mathrm{CH} H_{B 2}\right), 1.45\left(\mathrm{t}, J=7.1 \mathrm{~Hz}, 3 \mathrm{H}, \mathrm{CH}_{3}\right), 1.16(\mathrm{~d}, J$ $\left.=6.9 \mathrm{~Hz}, 3 \mathrm{H}, \mathrm{CH}_{3}\right)$. 
${ }^{13} \mathrm{C}$ NMR $(101 \mathrm{MHz}$, Chloroform- $d) \delta 212.0(\mathrm{C}=\mathrm{S}), 175.9(\mathrm{C}=\mathrm{O}), 153.1\left(\mathrm{C}_{\mathrm{Ar}}\right), 150.0\left(\mathrm{CH}_{\mathrm{Ar}}\right)$, 140.1 $\left(\mathrm{CH}_{\mathrm{Ar}}\right), 131.2\left(\mathrm{C}_{\mathrm{Ar}}\right), 125.6\left(\mathrm{CH}_{\mathrm{Ar}}\right), 116.9(\mathrm{CN}), 71.3\left(\mathrm{CH}_{2}\right), 45.3(\mathrm{~S}-\mathrm{CH}), 42.4\left(\mathrm{SO}_{2} \mathrm{CH}_{3}\right)$, $37.7(\mathrm{CH}), 35.6\left(\mathrm{CH}_{2}\right), 24.8\left(\mathrm{CH}_{2}\right), 17.6\left(\mathrm{CH}_{3}\right), 13.8\left(\mathrm{CH}_{3}\right)$.

IR (neat), $v\left(\mathrm{~cm}^{-1}\right): 1706,1454,1356,1224,1150,1107,1041,959,775,732,547$.

HRMS (ESI) m/z: $[\mathrm{M}+\mathrm{H}]^{+}$Calcd for $\mathrm{C}_{16} \mathrm{H}_{21} \mathrm{ClN}_{3} \mathrm{O}_{4} \mathrm{~S}_{3}$ 450.0377; Found 450.0378 .

$\mathrm{R}_{\mathrm{f}}=0.42(\mathrm{PE} / \mathrm{EtOAc}=40 \%)$. 


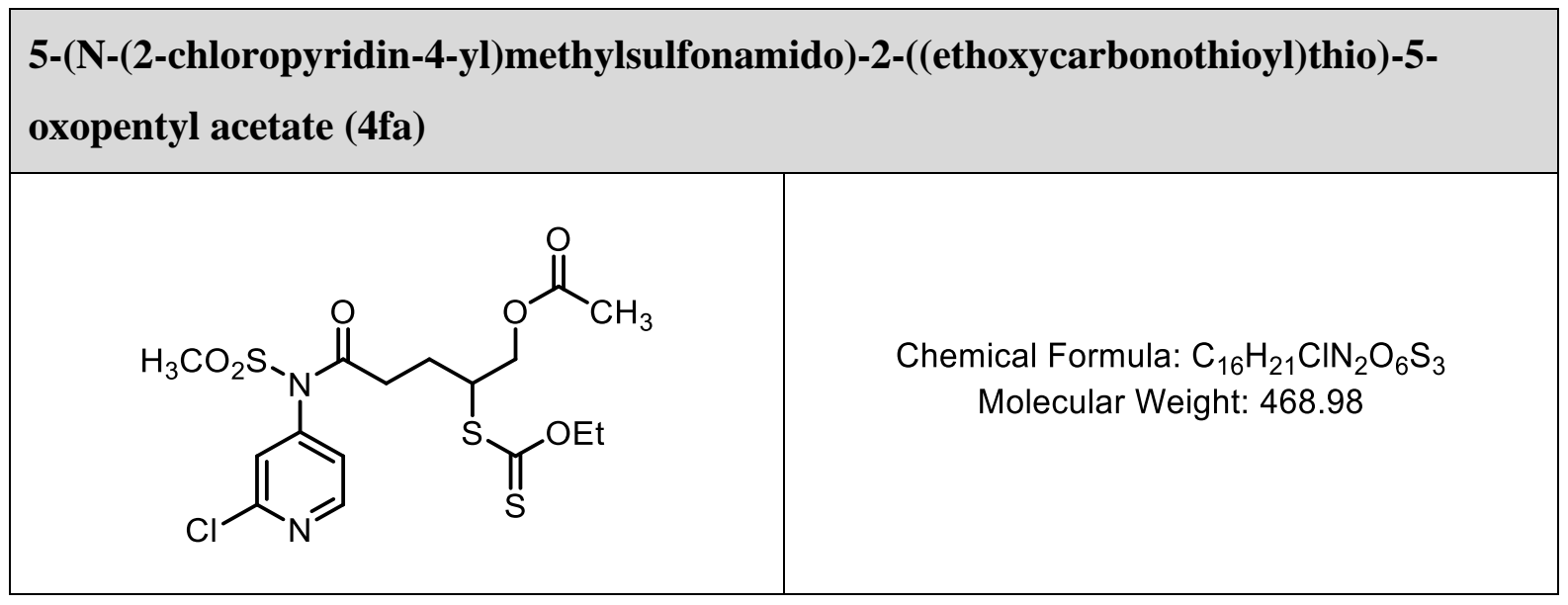

Following the general procedure $\mathrm{D}$, the reaction was carried out with the solution of $\mathbf{3 f}$ (300 $\mathrm{mg}, 0.81 \mathrm{mmol}, 1$ eq.) and allyl acetate $(0.18 \mathrm{~mL}, 1.63 \mathrm{mmol}, 2$ eq. $)$ in EtOAc $(0.82 \mathrm{~mL})$ with 0.15 eq. of DLP (48 mg). Flash column chromatography on silica gel (eluent: PE/EtOAc $=0 \%$ $\rightarrow 10 \% \rightarrow 40 \%)$ afforded target product $\mathbf{4 f a}(275 \mathrm{mg}, 72 \%)$ as a colorless oil.

${ }^{1} \mathrm{H}$ NMR (400 MHz, Chloroform- $d$ ) $\delta 8.54\left(\mathrm{dd}, J=5.3,0.6 \mathrm{~Hz}, 1 \mathrm{H}, \mathrm{CH}_{\mathrm{Ar}}\right.$ ), 7.29 (dd, $J=1.8$, $\left.0.6 \mathrm{~Hz}, 1 \mathrm{H}, \mathrm{CH}_{\mathrm{Ar}}\right), 7.19\left(\mathrm{dd}, J=5.3,1.8 \mathrm{~Hz}, 1 \mathrm{H}, \mathrm{CH}_{\mathrm{Ar}}\right), 4.65\left(\mathrm{q}, J=7.1 \mathrm{~Hz}, 2 \mathrm{H}, \mathrm{CH}_{2}\right), 4.28$ $\left(\mathrm{dd}, J=11.5,4.9 \mathrm{~Hz}, 1 \mathrm{H}, \mathrm{CH} H_{A l} \mathrm{H}\right), 4.19\left(\mathrm{dd}, J=11.4,6.1 \mathrm{~Hz}, 1 \mathrm{H}, \mathrm{CH} H_{A 2}\right), 4.04-3.95(\mathrm{~m}, 1 \mathrm{H}$, $\mathrm{S}-\mathrm{CH}), 3.50$ (s, 3H, $\left.\mathrm{SO}_{2} \mathrm{CH}_{3}\right), 2.36-2.29\left(\mathrm{~m}, 2 \mathrm{H}, \mathrm{CH}_{2}\right), 2.29-2.18\left(\mathrm{~m}, 1 \mathrm{H}, \mathrm{CH}_{B 1} \mathrm{H}\right), 2.05$ (s, $\left.3 \mathrm{H}, \mathrm{CH}_{3}\right), 1.91-1.80\left(\mathrm{~m}, 1 \mathrm{H}, \mathrm{CH}_{B 2}\right), 1.43\left(\mathrm{t}, J=7.1 \mathrm{~Hz}, 3 \mathrm{H}, \mathrm{CH}_{3}\right)$.

${ }^{13} \mathrm{C}$ NMR (101 MHz, Chloroform- $\left.d\right) \delta 213.4(\mathrm{C}=\mathrm{S}), 171.6(\mathrm{C}=\mathrm{O}), 170.7(\mathrm{C}=\mathrm{O}), 153.3\left(\mathrm{C}_{\mathrm{Ar}}\right)$, $151.5\left(\mathrm{CH}_{\mathrm{Ar}}\right), 145.2\left(\mathrm{C}_{\mathrm{Ar}}\right), 125.6\left(\mathrm{CH}_{\mathrm{Ar}}\right), 123.7\left(\mathrm{CH}_{\mathrm{Ar}}\right), 71.1\left(\mathrm{CH}_{2}\right), 65.8\left(\mathrm{CH}_{2}\right), 49.1(\mathrm{CH})$, $42.8\left(\mathrm{SO}_{2} \mathrm{CH}_{3}\right), 34.0\left(\mathrm{CH}_{2}\right), 25.8\left(\mathrm{CH}_{2}\right), 20.9\left(\mathrm{CH}_{3}\right), 13.9\left(\mathrm{CH}_{3}\right)$.

IR (neat), $v\left(\mathrm{~cm}^{-1}\right): 1732,1713,1579,1555,1461,1356,1221,1148,1038,964,787,734$.

HRMS (ESI) m/z: $[\mathrm{M}+\mathrm{H}]^{+}$Calcd for $\mathrm{C}_{16} \mathrm{H}_{22} \mathrm{ClN}_{2} \mathrm{O}_{6} \mathrm{~S}_{3} 469.0323$; Found 469.0327.

$\mathrm{R}_{\mathrm{f}}=0.36(\mathrm{PE} / \mathrm{EtOAc}=40 \%)$. 


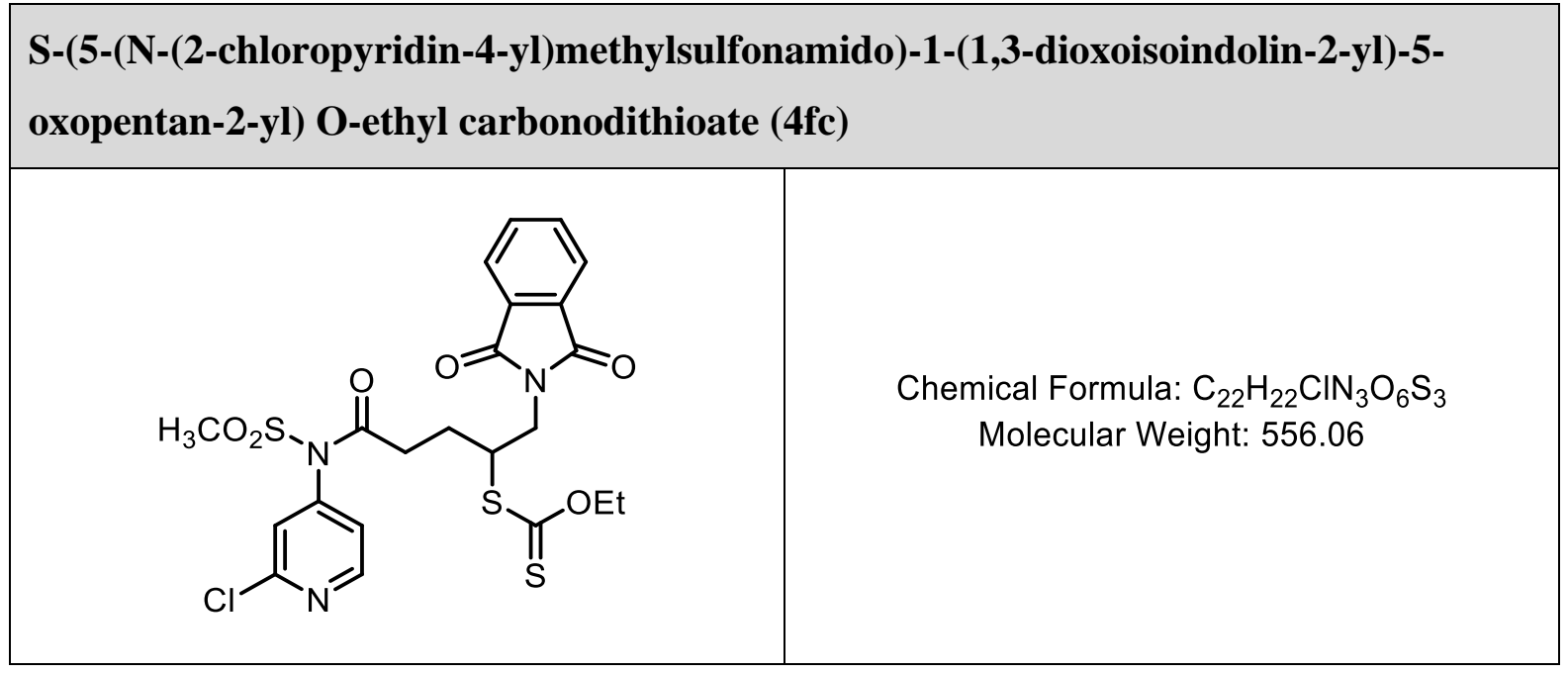

Following the general procedure D, the reaction was carried out with the solution of $\mathbf{3 f}$ (307 $\mathrm{mg}, 0.83 \mathrm{mmol}, 1$ eq.) and allyl phthalimide (312 mg, $1.66 \mathrm{mmol}, 2$ eq.) in EtOAc (0.83 mL) with 0.1 eq. of DLP (35 mg). Flash column chromatography on silica gel (eluent: PE/EtOAc = $0 \% \rightarrow 25 \% \rightarrow 45 \%)$ afforded target product $\mathbf{4 f c}(417 \mathrm{mg}, 90 \%)$ as a white foam.

${ }^{1} \mathrm{H}$ NMR (400 MHz, Chloroform- $d$ ) $\delta 8.53\left(\mathrm{~d}, J=5.2 \mathrm{~Hz}, 1 \mathrm{H}, \mathrm{CH}_{\mathrm{Ar}}\right), 7.88-7.82(\mathrm{~m}, 2 \mathrm{H}$, $\mathrm{CH}_{\text {Phth }}$ ), 7.76 - 7.71 (m, 2H, $\left.\mathrm{CH}_{\mathrm{Phth}}\right), 7.31$ (d, $\left.J=1.8 \mathrm{~Hz}, 1 \mathrm{H}, \mathrm{CH}_{\mathrm{Ar}}\right), 7.21$ (dd, $J=5.2,1.8 \mathrm{~Hz}$, $\left.1 \mathrm{H}, \mathrm{CH}_{\mathrm{Ar}}\right), 4.60\left(\mathrm{q}, J=7.1 \mathrm{~Hz}, 2 \mathrm{H}, \mathrm{CH}_{2}\right), 4.18-4.08(\mathrm{~m}, 1 \mathrm{H}, \mathrm{S}-\mathrm{CH}), 3.95(\mathrm{dd}, J=14.0,7.2$ $\left.\mathrm{Hz}, 1 \mathrm{H}, \mathrm{CH}_{A I} \mathrm{H}\right), 3.87\left(\mathrm{dd}, J=14.0,7.5 \mathrm{~Hz}, 1 \mathrm{H}, \mathrm{CH}_{A 2}\right), 3.49\left(\mathrm{~s}, 3 \mathrm{H}, \mathrm{SO}_{2} \mathrm{CH}_{3}\right), 2.36$ (t, $J=$ $\left.6.8 \mathrm{~Hz}, 2 \mathrm{H}, \mathrm{CH}_{2}\right), 2.28-2.11\left(\mathrm{~m}, 1 \mathrm{H}, \mathrm{CH}_{B 1} \mathrm{H}\right), 1.95-1.79\left(\mathrm{~m}, 1 \mathrm{H}, \mathrm{CH} H_{B 2}\right), 1.40(\mathrm{t}, J=7.1$ $\left.\mathrm{Hz}, 3 \mathrm{H}, \mathrm{CH}_{3}\right)$.

${ }^{13} \mathrm{C}$ NMR (101 MHz, Chloroform- $\left.d\right) \delta 212.6(\mathrm{C}=\mathrm{S}), 171.6(\mathrm{C}=\mathrm{O}), 168.2(\mathrm{C}=\mathrm{O}), 153.2\left(\mathrm{C}_{\mathrm{Ar}}\right)$, $151.5\left(\mathrm{CH}_{\mathrm{Ar}}\right), 145.2\left(\mathrm{C}_{\mathrm{Ar}}\right), 134.4\left(2 \mathrm{C}, \mathrm{CH}_{\mathrm{Phth}}\right), 131.9\left(2 \mathrm{C}, \mathrm{C}_{\mathrm{Phth}}\right), 125.7\left(\mathrm{CH}_{\mathrm{Ar}}\right), 123.8\left(\mathrm{CH}_{\mathrm{Ar}}\right)$, 123.7 (2C, $\left.\mathrm{CH}_{\text {Phth }}\right), 71.0\left(\mathrm{CH}_{2}\right), 48.9(\mathrm{~S}-\mathrm{CH}), 42.8\left(\mathrm{SO}_{2} \mathrm{CH}_{3}\right), 40.8\left(\mathrm{CH}_{2}\right), 33.9\left(\mathrm{CH}_{2}\right), 26.3$ $\left(\mathrm{CH}_{2}\right), 13.8\left(\mathrm{CH}_{3}\right)$.

IR (neat), $v\left(\mathrm{~cm}^{-1}\right): 1772,1713,1579,1395,1358,1223,1154,1047,967,751,720$.

HRMS (ESI) m/z: [M + H] $]^{+}$Calcd for $\mathrm{C}_{22} \mathrm{H}_{23} \mathrm{ClN}_{3} \mathrm{O}_{6} \mathrm{~S}_{3}$ 556.0432; Found 556.0433.

$\mathrm{R}_{\mathrm{f}}=0.26(\mathrm{PE} / \mathrm{EtOAc}=40 \%)$. 
5-(N-(6-chloropyridin-2-yl)methylsulfonamido)-2-((ethoxycarbonothioyl)thio)-5oxopentyl acetate (4ga)<smiles></smiles>

Chemical Formula: $\mathrm{C}_{16} \mathrm{H}_{21} \mathrm{ClN}_{2} \mathrm{O}_{6} \mathrm{~S}_{3}$ Molecular Weight: 468.98

Following the general procedure $\mathrm{D}$, the reaction was carried out with the solution of $3 \mathrm{~g}$ (300 $\mathrm{mg}, 0.81 \mathrm{mmol}, 1$ eq.) and allyl acetate $(0.18 \mathrm{~mL}, 1.63 \mathrm{mmol}, 2$ eq. $)$ in EtOAc $(0.82 \mathrm{~mL})$ with 0.15 eq. of DLP (48 mg). Flash column chromatography on silica gel (eluent: PE/EtOAc $=0 \%$ $\rightarrow 10 \% \rightarrow 30 \%)$ afforded target product 4 ga $(231 \mathrm{mg}, 61 \%)$ as a colorless oil.

${ }^{1} \mathrm{H}$ NMR (400 MHz, Chloroform- $d$ ) $\delta 7.85\left(\mathrm{dd}, J=8.0,7.6 \mathrm{~Hz}, 1 \mathrm{H}, \mathrm{CH}_{\mathrm{Ar}}\right), 7.47(\mathrm{dd}, J=8.0$, $\left.0.8 \mathrm{~Hz}, 1 \mathrm{H}, \mathrm{CH}_{\mathrm{Ar}}\right), 7.39\left(\mathrm{dd}, J=7.6,0.8 \mathrm{~Hz}, 1 \mathrm{H}, \mathrm{CH}_{\mathrm{Ar}}\right), 4.62\left(\mathrm{~d}, J=7.1 \mathrm{~Hz}, 2 \mathrm{H}, \mathrm{CH}_{2}\right), 4.26$ $\left(\mathrm{dd}, J=11.4,4.8 \mathrm{~Hz}, 1 \mathrm{H}, \mathrm{CH}_{A l} \mathrm{H}\right), 4.17\left(\mathrm{dd}, J=11.4,6.2 \mathrm{~Hz}, 1 \mathrm{H}, \mathrm{CH} H_{A 2}\right), 3.97-3.88(\mathrm{~m}, 1 \mathrm{H}$, $\mathrm{S}-\mathrm{CH}), 3.47$ (s, 3H, $\left.\mathrm{SO}_{2} \mathrm{CH}_{3}\right), 2.43-2.29\left(\mathrm{~m}, 2 \mathrm{H}, \mathrm{CH}_{2}\right), 2.28-2.17\left(\mathrm{~m}, 1 \mathrm{H}, \mathrm{CH}_{B l} \mathrm{H}\right), 2.06$ (s, $\left.3 \mathrm{H}, \mathrm{CH}_{3}\right), 1.97-1.84\left(\mathrm{~m}, 1 \mathrm{H}, \mathrm{CH} H_{B 2}\right), 1.41\left(\mathrm{t}, J=7.1 \mathrm{~Hz}, 3 \mathrm{H}, \mathrm{CH}_{3}\right)$.

${ }^{13} \mathrm{C}$ NMR (101 MHz, Chloroform- $\left.d\right) \delta 212.7(\mathrm{C}=\mathrm{S}), 172.4(\mathrm{C}=\mathrm{O}), 170.7(\mathrm{C}=\mathrm{O}), 151.2\left(\mathrm{C}_{\mathrm{Ar}}\right)$, $148.8\left(\mathrm{C}_{\mathrm{Ar}}\right), 141.4\left(\mathrm{CH}_{\mathrm{Ar}}\right), 126.1\left(\mathrm{CH}_{\mathrm{Ar}}\right), 124.7\left(\mathrm{CH}_{\mathrm{Ar}}\right), 70.7\left(\mathrm{CH}_{2}\right), 65.7\left(\mathrm{CH}_{2}\right), 48.6(\mathrm{~S}-\mathrm{CH})$, $41.9\left(\mathrm{SO}_{2} \mathrm{CH}_{3}\right), 34.2\left(\mathrm{CH}_{2}\right), 25.8\left(\mathrm{CH}_{2}\right), 20.9\left(\mathrm{CH}_{3}\right), 13.9\left(\mathrm{CH}_{3}\right)$.

IR (neat), $v\left(\mathrm{~cm}^{-1}\right): 1739,1715,1579,1562,1429,1358,1225,1162,1043,967,753$.

HRMS (ESI) m/z: $[\mathrm{M}+\mathrm{H}]^{+}$Calcd for $\mathrm{C}_{16} \mathrm{H}_{22} \mathrm{ClN}_{2} \mathrm{O}_{6} \mathrm{~S}_{3}$ 469.0323; Found 469.0324.

$\mathrm{R}_{\mathrm{f}}=0.51(\mathrm{PE} / \mathrm{EtOAc}=40 \%)$. 


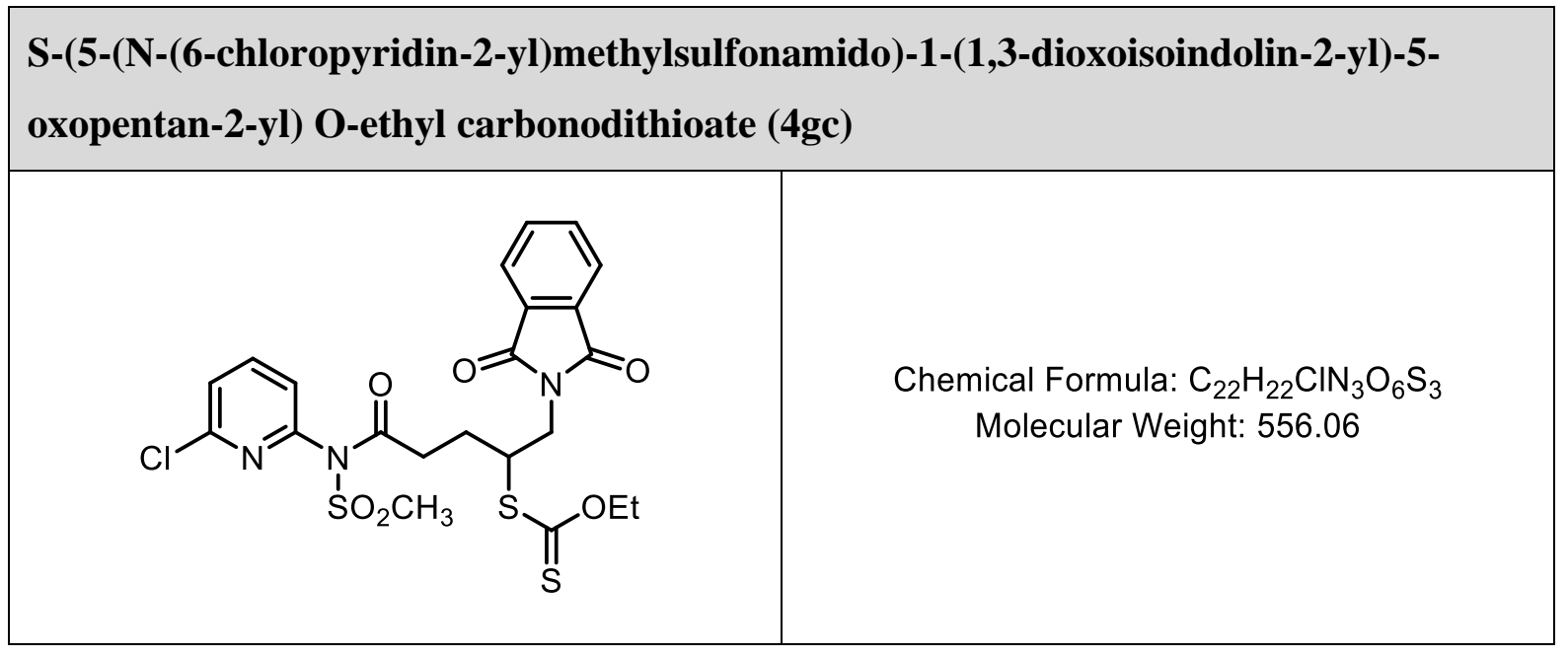

Following the general procedure D, the reaction was carried out with the solution of $\mathbf{3 g}$ (200 mg, 0.54 mmol, 1 eq.) and allyl phthalimide (203 mg, 1.08 mmol, 2 eq.) in EtOAc (0.54 mL) with 0.1 eq. of DLP $(22 \mathrm{mg})$. Flash column chromatography on silica gel (eluent: PE/EtOAc = $0 \% \rightarrow 25 \% \rightarrow 45 \%)$ afforded target product $4 \mathrm{gc}(265 \mathrm{mg}, 88 \%)$ as a white foam.

${ }^{1} \mathrm{H}$ NMR (400 MHz, Chloroform- $d$ ) $\delta 7.87-7.79\left(\mathrm{~m}, 3 \mathrm{H}, \mathrm{CH}_{\mathrm{Ar}}\right.$ and $\left.\mathrm{CH}_{\mathrm{Phth}}\right), 7.76-7.68(\mathrm{~m}$, $\left.2 \mathrm{H}, \mathrm{CH}_{\text {Phth }}\right), 7.45\left(\mathrm{dd}, J=8.0,0.6 \mathrm{~Hz}, 1 \mathrm{H}, \mathrm{CH}_{\mathrm{Ar}}\right), 7.40\left(\mathrm{dd}, J=7.7,0.6 \mathrm{~Hz}, 1 \mathrm{H}, \mathrm{CH}_{\mathrm{Ar}}\right), 4.57$ (q, $\left.J=7.2 \mathrm{~Hz}, 2 \mathrm{H}, \mathrm{CH}_{2}\right), 4.12-4.03(\mathrm{~m}, 1 \mathrm{H}, \mathrm{S}-\mathrm{CH}), 3.94\left(\mathrm{dd}, J=14.0,7.4 \mathrm{~Hz}, 1 \mathrm{H}, \mathrm{CH}_{A I} \mathrm{H}\right), 3.85$ $\left(\mathrm{dd}, J=14.0,7.3 \mathrm{~Hz}, 1 \mathrm{H}, \mathrm{CH} H_{A 2}\right), 3.45\left(\mathrm{~s}, 3 \mathrm{H}, \mathrm{SO}_{2} \mathrm{CH}_{3}\right), 2.49-2.27\left(\mathrm{~m}, 2 \mathrm{H}, \mathrm{CH}_{2}\right), 2.23-$ $2.12\left(\mathrm{~m}, 1 \mathrm{H}, \mathrm{CH}_{B 1} \mathrm{H}\right), 1.96-1.82\left(\mathrm{~m}, 1 \mathrm{H}, \mathrm{CH} H_{B 2}\right), 1.40\left(\mathrm{t}, J=7.1 \mathrm{~Hz}, 3 \mathrm{H}, \mathrm{CH}_{3}\right)$.

${ }^{13} \mathrm{C}$ NMR (101 MHz, Chloroform- $\left.d\right) \delta 212.1(\mathrm{C}=\mathrm{S}), 172.3(\mathrm{C}=\mathrm{O}), 168.1(\mathrm{C}=\mathrm{O}), 151.2\left(\mathrm{C}_{\mathrm{Ar}}\right)$, $148.8\left(\mathrm{C}_{\mathrm{Ar}}\right), 141.4\left(\mathrm{CH}_{\mathrm{Ar}}\right), 134.3\left(2 \mathrm{C}, \mathrm{CH}_{\mathrm{Phth}}\right), 131.9$ (2C, $\left.\mathrm{C}_{\mathrm{Phth}}\right), 126.0\left(\mathrm{CH}_{\mathrm{Ar}}\right), 124.7\left(\mathrm{CH}_{\mathrm{Ar}}\right)$, 123.6 (2C, $\left.\mathrm{CH}_{\text {Phth }}\right), 70.6\left(\mathrm{CH}_{2}\right), 48.6(\mathrm{~S}-\mathrm{CH}), 41.9\left(\mathrm{SO}_{2} \mathrm{CH}_{3}\right), 40.9\left(\mathrm{CH}_{2}\right), 34.1\left(\mathrm{CH}_{2}\right), 26.4$ $\left(\mathrm{CH}_{2}\right), 13.8\left(\mathrm{CH}_{3}\right)$.

IR (neat), $v\left(\mathrm{~cm}^{-1}\right): 1710,1498,1393,1355,1220,1150,1043,965,726,714,607$.

HRMS (ESI) m/z: $[\mathrm{M}+\mathrm{H}]^{+}$Calcd for $\mathrm{C}_{22} \mathrm{H}_{23} \mathrm{ClN}_{3} \mathrm{O}_{6} \mathrm{~S}_{3}$ 556.0432; Found 556.0432.

$\mathrm{R}_{\mathrm{f}}=0.26(\mathrm{PE} / \mathrm{EtOAc}=40 \%)$. 
<smiles>CC(=O)OCC1CCC(=O)Nc2ccc(Cl)nc21</smiles>

Chemical Formula: $\mathrm{C}_{12} \mathrm{H}_{13} \mathrm{CIN}_{2} \mathrm{O}_{3}$ Molecular Weight: 268.70

Following the general procedure $\mathrm{E}$, the reaction was carried out with the solution of $4 \mathbf{a a}$ (590 $\mathrm{mg}, 1.26 \mathrm{mmol}, 1$ eq.) in chlorobenzene $(63 \mathrm{~mL})$ with 5 eq. of DTBP $(1.20 \mathrm{~mL})$ for $1.5 \mathrm{~h}$. Flash column chromatography on silica gel (eluent: PE/EtOAc $=40 \% \rightarrow 70 \%$ ) afforded target product 5aa $(217 \mathrm{mg}, 64 \%)$ as a white solid.

${ }^{1} \mathrm{H}$ NMR (400 MHz, Chloroform- $d$ ) $\delta 9.29$ (s, 1H, NH), 7.32 (d, $J=8.3 \mathrm{~Hz}, 1 \mathrm{H}, \mathrm{CH}_{\mathrm{Ar}}$ ), 7.21 $\left(\mathrm{d}, J=8.3 \mathrm{~Hz}, 1 \mathrm{H}, \mathrm{CH}_{\mathrm{Ar}}\right), 4.70\left(\mathrm{dd}, J=11.2,6.3 \mathrm{~Hz}, 1 \mathrm{H}, \mathrm{OCH}_{A I} \mathrm{H}\right), 4.37(\mathrm{dd}, J=11.2,7.5 \mathrm{~Hz}$, $\left.1 \mathrm{H}, \mathrm{OCH} H_{A 2}\right), 3.52-3.41(\mathrm{~m}, 1 \mathrm{H}, \mathrm{CH}), 2.64-2.50\left(\mathrm{~m}, 1 \mathrm{H}, \mathrm{CH}_{B 1} \mathrm{H}\right), 2.43-2.34(\mathrm{~m}, 2 \mathrm{H}$, $\left.\mathrm{CH}_{2}\right), 2.02\left(\mathrm{~s}, 3 \mathrm{H}, \mathrm{CH}_{3}\right), 2.07-1.93\left(\mathrm{~m}, 1 \mathrm{H}, \mathrm{CHH}_{B 2}\right)$.

${ }^{13} \mathrm{C}$ NMR $(101 \mathrm{MHz}$, Chloroform- $d) \delta 175.4(\mathrm{C}=\mathrm{O}), 171.1(\mathrm{C}=\mathrm{O}), 154.0\left(\mathrm{C}_{\mathrm{Ar}}\right), 147.1\left(\mathrm{C}_{\mathrm{Ar}}\right)$, $133.2\left(\mathrm{C}_{\mathrm{Ar}}\right), 131.9\left(\mathrm{CH}_{\mathrm{Ar}}\right), 123.1\left(\mathrm{CH}_{\mathrm{Ar}}\right), 64.8\left(\mathrm{CH}_{2}\right), 40.2(\mathrm{CH}), 32.4\left(\mathrm{CH}_{2}\right), 31.3\left(\mathrm{CH}_{2}\right), 21.0$ $\left(\mathrm{CH}_{3}\right)$.

IR (neat), $v\left(\mathrm{~cm}^{-1}\right): 3036,2930,1733,1681,1435,1364,1252,1196,1159,1121,1027,936$, $919,828,798,767$.

HRMS (ESI) m/z: [M + H] $]^{+}$Calcd for $\mathrm{C}_{12} \mathrm{H}_{14} \mathrm{ClN}_{2} \mathrm{O}_{3}$ 269.0687; Found 269.0693.

$\mathrm{R}_{\mathrm{f}}=0.26(\mathrm{PE} / \mathrm{EtOAc}=70 \%)$.

m.p. $=176-180^{\circ} \mathrm{C}$. 
<smiles>N#CCC1CCC(=O)Nc2ccc(Cl)nc21</smiles>

Following the general procedure $\mathrm{E}$, the reaction was carried out with the solution of 4ab (280 $\mathrm{mg}, 0.64 \mathrm{mmol}, 1$ eq.) in chlorobenzene $(65 \mathrm{~mL})$ with 5 eq. of DTBP $(0.60 \mathrm{~mL})$ for $3 \mathrm{~h}$. Flash column chromatography on silica gel (eluent: PE/EtOAc $=50 \% \rightarrow 70 \%$ ) afforded target product 5ab (90 mg, 60\%) as a white solid.

${ }^{1} \mathrm{H}$ NMR $\left(400 \mathrm{MHz}, \mathrm{DMSO}-d_{6}\right) \delta 9.80(\mathrm{~s}, 1 \mathrm{H}, \mathrm{NH}), 7.47-7.41\left(\mathrm{~m}, 2 \mathrm{H}, \mathrm{CH}_{\mathrm{Ar}}\right), 3.49-3.36$ (m, 1H, CH), $2.96\left(\mathrm{dd}, J=16.7,7.5 \mathrm{~Hz}, 1 \mathrm{H}, \mathrm{CH}_{A l} \mathrm{H}\right), 2.86\left(\mathrm{dd}, J=16.7,6.0 \mathrm{~Hz}, 1 \mathrm{H}, \mathrm{CH} H_{A 2}\right)$, $2.49-2.41\left(\mathrm{~m}, 1 \mathrm{H}, \mathrm{CH}_{B l} \mathrm{H}\right), 2.37-2.25\left(\mathrm{~m}, 1 \mathrm{H}, \mathrm{C} H_{C l} \mathrm{H}\right), 2.23-2.13\left(\mathrm{~m}, 1 \mathrm{H}, \mathrm{CH} H_{C 2}\right), 1.99$ $-1.86\left(\mathrm{~m}, 1 \mathrm{H}, \mathrm{CH} H_{B 2}\right)$.

${ }^{13} \mathrm{C}$ NMR $\left(101 \mathrm{MHz}, \mathrm{DMSO}-d_{6}\right) \delta 173.5(\mathrm{C}=\mathrm{O}), 153.9\left(\mathrm{C}_{\mathrm{Ar}}\right), 145.5\left(\mathrm{C}_{\mathrm{Ar}}\right), 135.4\left(\mathrm{C}_{\mathrm{Ar}}\right), 133.5$ $\left(\mathrm{CH}_{\mathrm{Ar}}\right), 124.2\left(\mathrm{CH}_{\mathrm{Ar}}\right), 120.7(\mathrm{CN}), 38.3(\mathrm{CH}), 33.9\left(\mathrm{CH}_{2}\right), 33.4\left(\mathrm{CH}_{2}\right), 20.1\left(\mathrm{CH}_{2}\right)$.

IR (neat), $v\left(\mathrm{~cm}^{-1}\right)$ : 3206, 2934, 1676, 1441, 1368, 1192, 1161, 1136, 927, 835, 766, 734. HRMS (ESI) m/z: [M + H $]^{+}$Calcd for $\mathrm{C}_{11} \mathrm{H}_{11} \mathrm{ClN}_{3} \mathrm{O}$ 236.0585; Found 236.0591.

$\mathrm{R}_{\mathrm{f}}=0.30(\mathrm{PE} / \mathrm{EtOAc}=70 \%)$.

m.p. $=217-218^{\circ} \mathrm{C}$. 


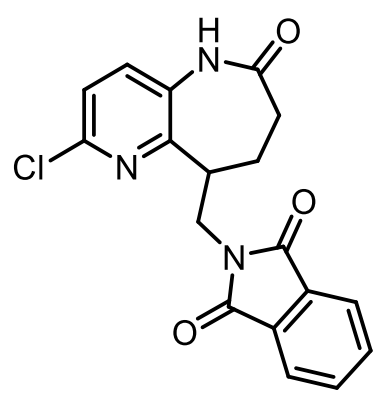

Chemical Formula: $\mathrm{C}_{18} \mathrm{H}_{14} \mathrm{ClN}_{3} \mathrm{O}_{3}$ Molecular Weight: 355.78

Following the general procedure E, the reaction was carried out with the solution of 4ac (250 $\mathrm{mg}, 0.45 \mathrm{mmol}, 1$ eq.) in chlorobenzene $(45 \mathrm{~mL})$ with 5 eq. of DTBP $(0.42 \mathrm{~mL})$ for $4 \mathrm{~h}$. Flash column chromatography on silica gel (eluent: PE/EtOAc $=50 \% \rightarrow 70 \%$ ) afforded target product 5ac (91 $\mathrm{mg}, 57 \%)$ as a white solid.

${ }^{1} \mathrm{H}$ NMR (400 MHz, DMSO-d6) $\delta 9.76$ (s, br, 1H, NH), 7.93 - 7.78 (m, 4H, CHPhth), 7.42 (s, $\left.2 \mathrm{H}, \mathrm{CH}_{\mathrm{Ar}}\right), 4.10-3.92\left(\mathrm{~m}, 2 \mathrm{H}, \mathrm{CH}_{2}\right), 3.59-3.46(\mathrm{~m}, 1 \mathrm{H}, \mathrm{CH}), 2.43-2.24\left(\mathrm{~m}, 2 \mathrm{H}, \mathrm{CH}_{2}\right), 2.18$ $-2.09\left(\mathrm{~m}, 1 \mathrm{H}, \mathrm{CH}_{A l} \mathrm{H}\right), 1.91-1.78\left(\mathrm{~m}, 1 \mathrm{H}, \mathrm{CH} H_{A 2}\right)$.

${ }^{13} \mathrm{C}$ NMR $\left(101 \mathrm{MHz}, \mathrm{DMSO}-d_{6}\right) \delta 172.7(\mathrm{C}=\mathrm{O}), 168.1(\mathrm{C}=\mathrm{O}), 153.7\left(\mathrm{C}_{\mathrm{Ar}}\right), 144.4\left(\mathrm{C}_{\mathrm{Ar}}\right), 134.5$ $\left(\mathrm{C}_{\mathrm{Ar}}\right), 134.3$ (2C, $\left.\mathrm{CH}_{\text {Phth }}\right), 132.4\left(\mathrm{CH}_{\mathrm{Ar}}\right), 131.7$ (2C, $\left.\mathrm{C}_{\text {Phth }}\right), 123.0\left(2 \mathrm{C}, \mathrm{CH}_{\text {Phth }}\right), 122.9\left(\mathrm{CH}_{\mathrm{Ar}}\right)$, $40.2\left(\mathrm{CH}_{2}\right), 38.9(\mathrm{CH}), 32.3\left(\mathrm{CH}_{2}\right), 31.2\left(\mathrm{CH}_{2}\right)$.

IR (neat), $v\left(\mathrm{~cm}^{-1}\right): 3218,2937,1771,1707,1676,1438,1399,1369,1228,1165,930,907$, 721.

HRMS (ESI) m/z: [M + H] $]^{+}$Calcd for $\mathrm{C}_{18} \mathrm{H}_{15} \mathrm{ClN}_{3} \mathrm{O}_{3}$ 356.0796; Found 356.0804.

$\mathrm{R}_{\mathrm{f}}=0.39(\mathrm{PE} / \mathrm{EtOAc}=70 \%)$.

Melting is not observed in the $20-225^{\circ} \mathrm{C}$ range. 
2-chloro-9-((trimethylsilyl)methyl)-5,7,8,9-tetrahydro-6H-pyrido[3,2-b]azepin-6-one (5ad)

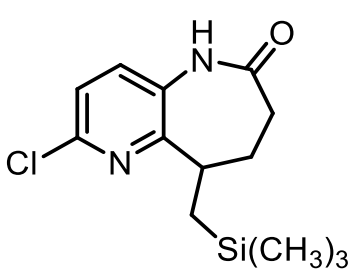

Chemical Formula: $\mathrm{C}_{13} \mathrm{H}_{19} \mathrm{CIN}_{2} \mathrm{OSi}$

Molecular Weight: 282.84

Following the general procedure E, the reaction was carried out with the solution of 4ad (303 $\mathrm{mg}, 0.63 \mathrm{mmol}, 1$ eq.) in chlorobenzene $(63 \mathrm{~mL})$ with 5 eq. of DTBP $(0.58 \mathrm{~mL})$ for $4 \mathrm{~h}$. Flash column chromatography on silica gel (eluent: PE/EtOAc $=30 \% \rightarrow 50 \%$ ) afforded target product 5ad (90 $\mathrm{mg}, 51 \%)$ as a white solid.

${ }^{1} \mathrm{H}$ NMR (400 MHz, Chloroform- $d$ ) $\delta 8.42(\mathrm{~s}, 1 \mathrm{H}, \mathrm{NH}), 7.26\left(\mathrm{~d}, J=8.2 \mathrm{~Hz}, 1 \mathrm{H}, \mathrm{CH}_{\mathrm{Ar}}\right), 7.19$ $\left(\mathrm{dd}, J=8.2,0.5 \mathrm{~Hz}, 1 \mathrm{H}, \mathrm{CH}_{\mathrm{Ar}}\right), 3.37-3.23(\mathrm{~m}, 1 \mathrm{H}, \mathrm{CH}), 2.58-2.44\left(\mathrm{~m}, 1 \mathrm{H}, \mathrm{CH}_{A l} \mathrm{H}\right), 2.39-$ $2.26\left(\mathrm{~m}, 2 \mathrm{H}, \mathrm{CH}_{2}\right), 2.03-1.87\left(\mathrm{~m}, 1 \mathrm{H}, \mathrm{CH} H_{A 2}\right), 1.46\left(\mathrm{dd}, J=14.7,8.2 \mathrm{~Hz}, 1 \mathrm{H}, \mathrm{CH}_{B 1} \mathrm{H}\right), 0.87$ (dd, $\left.J=14.7,6.3 \mathrm{~Hz}, 1 \mathrm{H}, \mathrm{CH} H_{B 2}\right),-0.07$ (s, 9H, $\left.\mathrm{Si}\left(\mathrm{CH}_{3}\right)_{3}\right)$.

${ }^{13} \mathrm{C}$ NMR (101 MHz, Chloroform- $\left.d\right) \delta 175.4(\mathrm{C}=\mathrm{O}), 158.2\left(\mathrm{C}_{\mathrm{Ar}}\right), 147.1\left(\mathrm{C}_{\mathrm{Ar}}\right), 132.9\left(\mathrm{C}_{\mathrm{Ar}}\right)$, $131.6\left(\mathrm{CH}_{\mathrm{Ar}}\right), 122.4\left(\mathrm{CH}_{\mathrm{Ar}}\right), 38.2(\mathrm{CH}), 37.6\left(\mathrm{CH}_{2}\right), 33.2\left(\mathrm{CH}_{2}\right), 19.1\left(\mathrm{CH}_{2}\right),-0.6(3 \mathrm{C}$, $\left.\mathrm{Si}\left(\mathrm{CH}_{3}\right)_{3}\right)$.

IR (neat), $v\left(\mathrm{~cm}^{-1}\right): 3202,2942,1671,1436,1363,1246,1164,1133,839,766$.

HRMS (ESI) m/z: $[\mathrm{M}+\mathrm{H}]^{+}$Calcd for $\mathrm{C}_{13} \mathrm{H}_{20} \mathrm{ClN}_{2} \mathrm{OSi} 283.1028$; Found 283.1038.

$\mathrm{R}_{\mathrm{f}}=0.41(\mathrm{PE} / \mathrm{EtOAc}=50 \%)$.

m.p. $=148-150^{\circ} \mathrm{C}($ crystallized from PE/EtOAc). 
2-chloro-6-oxo-6,7,8,9-tetrahydro-5H-pyrido[3,2-b]azepin-9-yl acetate (5ae)<smiles>CC(=O)OC1CCC(=O)Nc2ccc(Cl)nc21</smiles>

Chemical Formula: $\mathrm{C}_{11} \mathrm{H}_{11} \mathrm{CIN}_{2} \mathrm{O}_{3}$ Molecular Weight: 254.67

Following the general procedure E, the reaction was carried out with the solution of 4ae (457 $\mathrm{mg}, 1.00 \mathrm{mmol}, 1$ eq. $)$ in chlorobenzene $(100 \mathrm{~mL})$ with 5 eq. of DTBP $(0.93 \mathrm{~mL})$ for $2.5 \mathrm{~h}$. The mixture was washed with $\mathrm{Et}_{2} \mathrm{O}$ to afford target product 5ae (184 mg, 72\%) as a white solid.

${ }^{1} \mathrm{H}$ NMR (400 MHz, DMSO-d $\left.d_{6}\right) \delta 9.86(\mathrm{~s}, 1 \mathrm{H}, \mathrm{NH}), 7.45$ (d, $J=0.8 \mathrm{~Hz}, 2 \mathrm{H}, \mathrm{CH}_{\mathrm{Ar}}$ ), 5.74 (dd, $J=8.3,6.8 \mathrm{~Hz}, 1 \mathrm{H}, \mathrm{CH}), 2.61-2.53\left(\mathrm{~m}, 1 \mathrm{H}, \mathrm{CH}_{A l} \mathrm{H}\right), 2.39-2.29\left(\mathrm{~m}, 2 \mathrm{H}, \mathrm{CH}_{2}\right), 2.29-2.18$ $\left(\mathrm{m}, 1 \mathrm{H}, \mathrm{CH} H_{A 2}\right), 2.08\left(\mathrm{~s}, 3 \mathrm{H}, \mathrm{CH}_{3}\right)$.

${ }^{13} \mathrm{C}$ NMR $\left(101 \mathrm{MHz}, \mathrm{DMSO}-d_{6}\right) \delta 172.4(\mathrm{C}=\mathrm{O}), 169.5(\mathrm{C}=\mathrm{O}), 149.7\left(\mathrm{C}_{\mathrm{Ar}}\right), 144.2\left(\mathrm{C}_{\mathrm{Ar}}\right), 133.2$ $\left(\mathrm{C}_{\mathrm{Ar}}\right), 133.0\left(\mathrm{CH}_{\mathrm{Ar}}\right), 124.0\left(\mathrm{CH}_{\mathrm{Ar}}\right), 71.9(\mathrm{CH}), 31.4\left(\mathrm{CH}_{2}\right), 30.7\left(\mathrm{CH}_{2}\right), 20.8\left(\mathrm{CH}_{3}\right)$.

IR (neat), $v\left(\mathrm{~cm}^{-1}\right):$ 2935, 1728, 1664, 1442, 1369, 1236, 1161, 1138, $1048,764$.

HRMS (ESI) m/z: $[\mathrm{M}+\mathrm{H}]^{+}$Calcd for $\mathrm{C}_{11} \mathrm{H}_{12} \mathrm{ClN}_{2} \mathrm{O}_{3}$ 255.0531; Found 255.0537.

$\mathrm{R}_{\mathrm{f}}=0.17(\mathrm{PE} / \mathrm{EtOAc}=50 \%)$.

m.p $=220-223^{\circ} \mathrm{C}\left(\right.$ crystallized from $\left.\mathrm{Et}_{2} \mathrm{O}\right)$. 


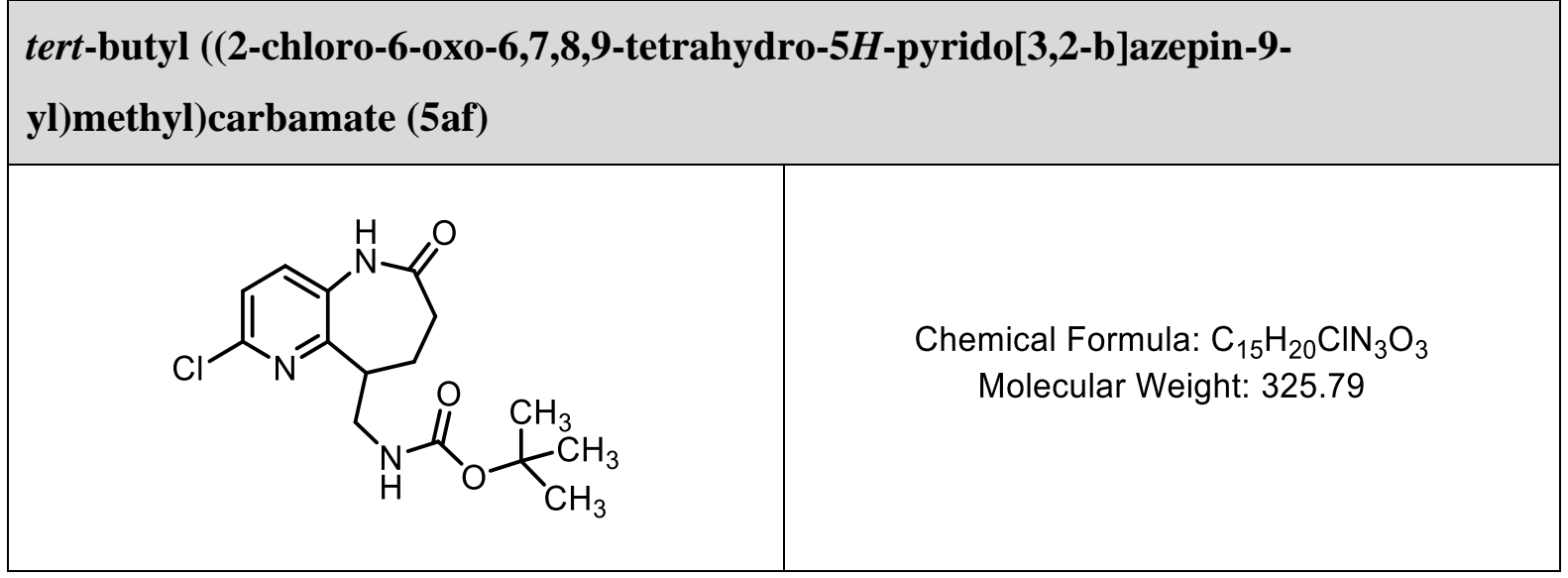

Following the general procedure E, the reaction was carried out with the solution of 4 af (268 $\mathrm{mg}, 0.59 \mathrm{mmol}, 1$ eq.) in chlorobenzene $(59 \mathrm{~mL})$ with 5 eq. of DTBP $(0.55 \mathrm{~mL})$ for $3 \mathrm{~h}$. Flash column chromatography on silica gel (eluent: PE/EtOAc $=50 \% \rightarrow 70 \%$ ) afforded target product 5af (94 mg, 63\%) as a yellow oil. For analytical purposes it was crystallized from $\mathrm{PE} / \mathrm{EtOAc}$ to yield white crystals.

${ }^{1} \mathrm{H}$ NMR (400 MHz, Chloroform- $d$ ) $\delta 9.16(\mathrm{~s}, 1 \mathrm{H}, \mathrm{NH}), 7.32\left(\mathrm{~d}, J=8.3 \mathrm{~Hz}, 1 \mathrm{H}, \mathrm{CH}_{\mathrm{Ar}}\right), 7.22$ $\left(\mathrm{d}, J=8.3 \mathrm{~Hz}, 1 \mathrm{H}, \mathrm{CH}_{\mathrm{Ar}}\right), 5.31(\mathrm{t}, J=6.5 \mathrm{~Hz}, 1 \mathrm{H}, \mathrm{NH}), 3.70-3.49\left(\mathrm{~m}, 2 \mathrm{H}, \mathrm{CH}_{2}\right), 3.42-3.24$ (m, 1H, CH), $2.53-2.38\left(\mathrm{~m}, 1 \mathrm{H}, \mathrm{CH}_{A l} \mathrm{H}\right), 2.38-2.26\left(\mathrm{~m}, 2 \mathrm{H}, \mathrm{CH}_{2}\right), 2.07-1.90(\mathrm{~m}, 1 \mathrm{H}$, $\left.\mathrm{CH} H_{A 2}\right), 1.40\left(\mathrm{~s}, 9 \mathrm{H}, \mathrm{CH}_{3}\right)$.

${ }^{13} \mathrm{C}$ NMR (101 MHz, Chloroform- $\left.d\right) \delta 175.4(\mathrm{C}=\mathrm{O}), 156.2\left(\mathrm{C}_{\mathrm{Ar}}\right), 155.6(\mathrm{C}=\mathrm{O}), 147.0\left(\mathrm{C}_{\mathrm{Ar}}\right)$, $133.8\left(\mathrm{C}_{\mathrm{Ar}}\right), 132.1\left(\mathrm{CH}_{\mathrm{Ar}}\right), 122.9\left(\mathrm{CH}_{\mathrm{Ar}}\right), 79.4(\mathrm{C}), 41.6(\mathrm{CH}), 41.1\left(\mathrm{CH}_{2}\right), 32.6\left(\mathrm{CH}_{2}\right), 31.4$ $\left(\mathrm{CH}_{2}\right), 28.5\left(3 \mathrm{C}, \mathrm{CH}_{3}\right)$.

IR (neat), $v\left(\mathrm{~cm}^{-1}\right): 3226,2976,2933,1680,1501,1439,1365,1277,1252,1164,1137,914$, 731.

HRMS (ESI) m/z: $[\mathrm{M}+\mathrm{H}]^{+}$Calcd for $\mathrm{C}_{15} \mathrm{H}_{21} \mathrm{ClN}_{3} \mathrm{O}_{3}$ 326.1266; Found 326.1273.

$\mathrm{R}_{\mathrm{f}}=0.31(\mathrm{PE} / \mathrm{EtOAc}=70 \%)$.

m.p. $=177-179^{\circ} \mathrm{C}($ crystallized from PE/EtOAc). 
2-chloro-9-((4,4,5,5-tetramethyl-1,3,2-dioxaborolan-2-yl)methyl)-5,7,8,9-tetrahydro$6 \mathrm{H}$-pyrido[3,2-b]azepin-6-one (5ag)<smiles>CC1(C)OB(CC2CCC(=O)Nc3ccc(Cl)nc32)OC1(C)C</smiles>

Chemical Formula: $\mathrm{C}_{16} \mathrm{H}_{22} \mathrm{BCIN}_{2} \mathrm{O}_{3}$ Molecular Weight: 336.62

Following the general procedure E, the reaction was carried out with the solution of $4 \mathbf{a g}$ (316 $\mathrm{mg}, 0.59 \mathrm{mmol}, 1$ eq.) in chlorobenzene $(59 \mathrm{~mL})$ with 5 eq. of DTBP $(0.55 \mathrm{~mL})$ for $4 \mathrm{~h}$. Flash column chromatography on silica gel (eluent: PE/EtOAc $=50 \% \rightarrow 70 \%$ ) afforded target product 5ag (102 mg, 52\%) as a white solid.

${ }^{1} \mathrm{H}$ NMR (400 MHz, Chloroform- $d$ ) $\delta 9.23(\mathrm{~s}, 1 \mathrm{H}, \mathrm{NH}), 7.26\left(\mathrm{~d}, J=8.2 \mathrm{~Hz}, 1 \mathrm{H}, \mathrm{CH}_{\mathrm{Ar}}\right), 7.13$ $\left(\mathrm{d}, J=8.2 \mathrm{~Hz}, 1 \mathrm{H}, \mathrm{CH}_{\mathrm{Ar}}\right), 3.48-3.36(\mathrm{~m}, 1 \mathrm{H}, \mathrm{CH}), 2.56-2.44\left(\mathrm{~m}, 1 \mathrm{H}, \mathrm{CH}_{A l} \mathrm{H}\right), 2.43-2.25$ $\left(\mathrm{m}, 2 \mathrm{H}, \mathrm{CH}_{2}\right), 2.08-1.96\left(\mathrm{~m}, 1 \mathrm{H}, \mathrm{CH} H_{A 2}\right), 1.43-1.32\left(\mathrm{~m}, 1 \mathrm{H}, \mathrm{CH}_{C l} \mathrm{H}\right), 1.18\left(\mathrm{~s}, 6 \mathrm{H}, 2 \times \mathrm{CH}_{3}\right)$, $1.10-1.04\left(\mathrm{~m}, 1 \mathrm{H}, \mathrm{CH} H_{C 2}\right), 1.09\left(\mathrm{~s}, 6 \mathrm{H}, 2 \mathrm{x} \mathrm{CH}_{3}\right)$.

${ }^{13} \mathrm{C}$ NMR (101 MHz, Chloroform- $\left.d\right) \delta 175.9(\mathrm{C}=\mathrm{O}), 157.9\left(\mathrm{C}_{\mathrm{Ar}}\right), 146.7\left(\mathrm{C}_{\mathrm{Ar}}\right), 133.0\left(\mathrm{C}_{\mathrm{Ar}}\right)$, $131.5\left(\mathrm{CH}_{\mathrm{Ar}}\right), 122.2\left(\mathrm{CH}_{\mathrm{Ar}}\right), 83.0(2 \mathrm{C}, \mathrm{C}-\mathrm{O}), 37.6(\mathrm{CH}), 36.7\left(\mathrm{CH}_{2}\right), 33.2\left(\mathrm{CH}_{2}\right), 25.0(2 \mathrm{C}$, $\left.\mathrm{CH}_{3}\right), 24.7\left(2 \mathrm{C}, \mathrm{CH}_{3}\right)$. B- $\mathrm{CH}_{2}$ signal is not observed.

IR (neat), $v\left(\mathrm{~cm}^{-1}\right): 3211,2977,1673,1438,1371,1318,1165,1143,967,921,846,735$.

HRMS (ESI) m/z: $[\mathrm{M}+\mathrm{H}]^{+}$Calcd for $\mathrm{C}_{16} \mathrm{H}_{23} \mathrm{BClN}_{2} \mathrm{O}_{3}$ 337.1315; Found 337.1495.

$\mathrm{R}_{\mathrm{f}}=0.59(\mathrm{PE} / \mathrm{EtOAc}=70 \%)$

m.p. $=198-200^{\circ} \mathrm{C}($ crystallized from PE/EtOAc $)$. 


\section{9-(2,2-bis(4,4,5,5-tetramethyl-1,3,2-dioxaborolan-2-yl)ethyl)-2-chloro-5,7,8,9- tetrahydro-6H-pyrido[3,2-b]azepin-6-one (5ah)}

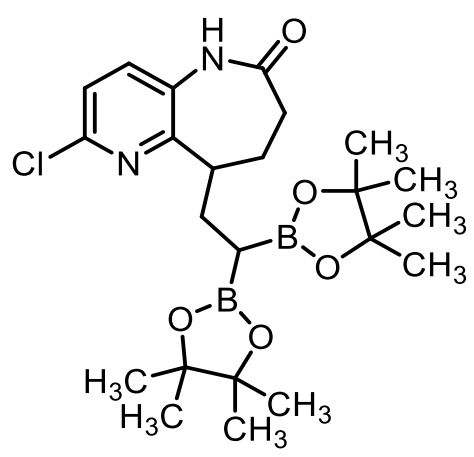

Chemical Formula: $\mathrm{C}_{23} \mathrm{H}_{35} \mathrm{~B}_{2} \mathrm{CIN}_{2} \mathrm{O}_{5}$ Molecular Weight: 476.61

Following the general procedure E, the reaction was carried out with the solution of $\mathbf{4 a h}$ (320 $\mathrm{mg}, 0.47 \mathrm{mmol}, 1$ eq.) in chlorobenzene ( $48 \mathrm{~mL})$ with 5 eq. of DTBP $(0.44 \mathrm{~mL})$ for $3.5 \mathrm{~h}$. Flash column chromatography on silica gel (eluent: $\mathrm{PE} / \mathrm{EtOAc}=40 \% \rightarrow 70 \%$ ) afforded target product 5ah (88 mg, 39\%) as a yellow oil.

${ }^{1} \mathrm{H}$ NMR (400 MHz, Chloroform- $d$ ) $\delta 8.54(\mathrm{~s}, 1 \mathrm{H}, \mathrm{NH}), 7.21\left(\mathrm{~d}, J=8.3 \mathrm{~Hz}, 1 \mathrm{H}, \mathrm{CH}_{\mathrm{Ar}}\right), 7.12$ $\left(\mathrm{d}, J=8.3 \mathrm{~Hz}, 1 \mathrm{H}, \mathrm{CH}_{\mathrm{Ar}}\right), 3.22-3.07(\mathrm{~m}, 1 \mathrm{H}, \mathrm{CH}), 2.56-2.42\left(\mathrm{~m}, 1 \mathrm{H}, \mathrm{CH}_{A l} \mathrm{H}\right), 2.38-2.18$ $\left(\mathrm{m}, 3 \mathrm{H}, \mathrm{CH}_{2}\right.$ and $\left.\mathrm{CH}_{B 1} \mathrm{H}\right), 1.97-1.79\left(\mathrm{~m}, 2 \mathrm{H}, \mathrm{CH}_{A 2}\right.$ and $\left.\mathrm{CH}_{B 2}\right), 1.18\left(\mathrm{~s}, 24 \mathrm{H}, \mathrm{CH}_{3}\right), 0.75$ (dd, $J=9.1,6.6 \mathrm{~Hz}, 1 \mathrm{H}, \mathrm{B}-\mathrm{CH})$.

${ }^{13} \mathrm{C}$ NMR $(101 \mathrm{MHz}$, Chloroform- $d) \delta 175.5(\mathrm{C}=\mathrm{O}), 157.3\left(\mathrm{C}_{\mathrm{Ar}}\right), 147.0\left(\mathrm{C}_{\mathrm{Ar}}\right), 133.1\left(\mathrm{C}_{\mathrm{Ar}}\right)$, $131.5\left(\mathrm{CH}_{\mathrm{Ar}}\right), 122.2\left(\mathrm{CH}_{\mathrm{Ar}}\right), 83.2(\mathrm{C}), 83.1(4 \mathrm{C}, \mathrm{C}-\mathrm{O}), 43.3(\mathrm{CH}), 33.8\left(\mathrm{CH}_{2}\right), 32.9\left(\mathrm{CH}_{2}\right), 27.1$ $\left(\mathrm{CH}_{2}\right), 24.6\left(4 \mathrm{C}, \mathrm{CH}_{3}\right), 24.8\left(4 \mathrm{C}, \mathrm{CH}_{3}\right)$. B-CH is not observed.

IR (neat), $v\left(\mathrm{~cm}^{-1}\right): 2977,1674,1437,1359,1310,1250,1135,967,848,734$.

HRMS (ESI) m/z: $[\mathrm{M}+\mathrm{H}]^{+}$Calcd for $\mathrm{C}_{23} \mathrm{H}_{36} \mathrm{~B}_{2} \mathrm{ClN}_{2} \mathrm{O}_{5}$ 477.2493; Found 477.2503.

$\mathrm{R}_{\mathrm{f}}=0.24(\mathrm{PE} / \mathrm{EtOAc}=50 \%)$. 


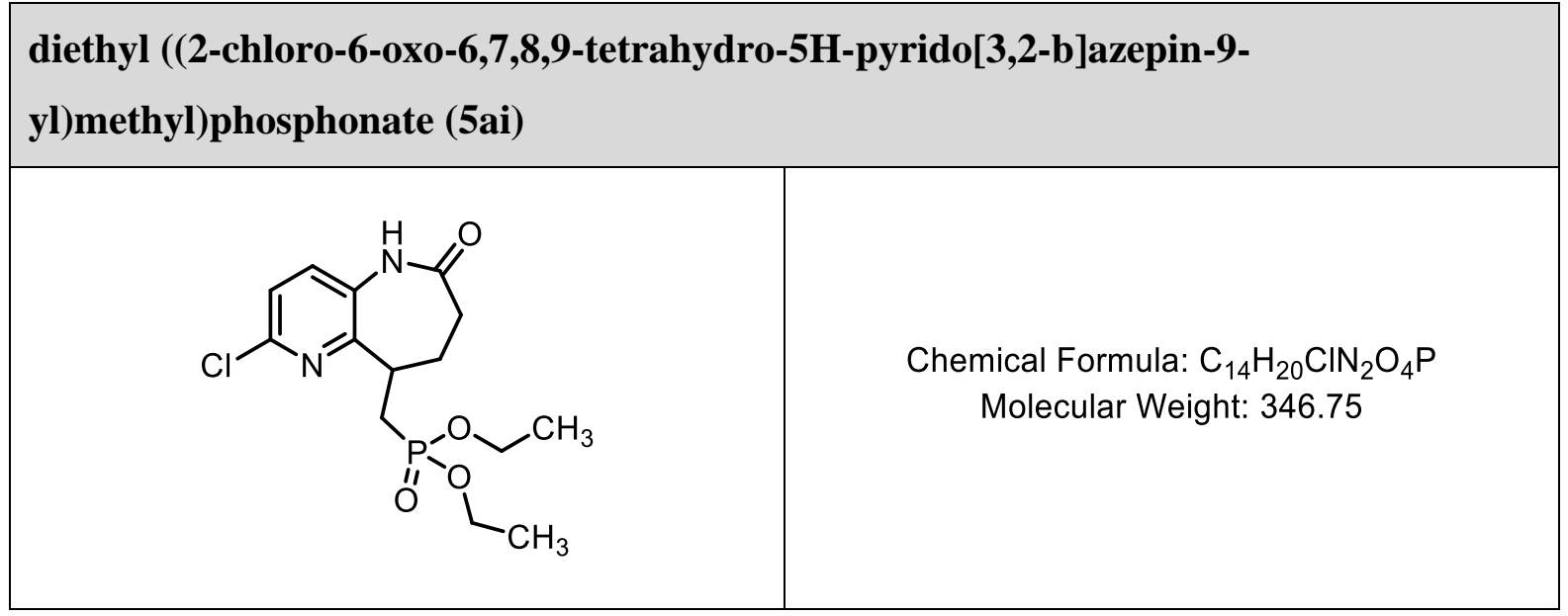

Following the general procedure E, the reaction was carried out with the solution of 4ai (244 $\mathrm{mg}, 0.45 \mathrm{mmol}, 1$ eq.) in chlorobenzene $(45 \mathrm{~mL})$ with 5 eq. of DTBP $(0.41 \mathrm{~mL})$ for $4.5 \mathrm{~h}$. Flash column chromatography on silica gel (eluent: EtOAc/MeOH =10\%) afforded target product 5ai (38 mg, 24\%) as a yellow oil.

${ }^{1} \mathrm{H}$ NMR (400 MHz, Acetone-d $\left.d_{6}\right) \delta 8.92(\mathrm{~s}, 1 \mathrm{H}, \mathrm{NH}), 7.49$ (d, $\left.J=8.3 \mathrm{~Hz}, 1 \mathrm{H}, \mathrm{CH}_{\mathrm{Ar}}\right), 7.35$ (d, $\left.J=8.3 \mathrm{~Hz}, 1 \mathrm{H}, \mathrm{CH}_{\mathrm{Ar}}\right), 4.14-3.96\left(\mathrm{~m}, 4 \mathrm{H}, 2 \mathrm{x} \mathrm{CH}_{2}\right), 3.61-3.48(\mathrm{~m}, 1 \mathrm{H}, \mathrm{CH}), 2.75-2.60$ (m, $2 \mathrm{H}, \mathrm{CH}_{A l} \mathrm{H}$ and $\left.\mathrm{CH}_{B l} \mathrm{H}\right), 2.42-2.29\left(\mathrm{~m}, 1 \mathrm{H}, \mathrm{CH}_{C l} \mathrm{H}\right), 2.27-2.17\left(\mathrm{~m}, 1 \mathrm{H}, \mathrm{CH} H_{C 2}\right), 2.09-$ $1.88\left(\mathrm{~m}, 2 \mathrm{H}, \mathrm{CH} H_{A 2}\right.$ and $\left.\mathrm{CH}_{B 2}\right), 1.22\left(\mathrm{t}, J=7.1 \mathrm{~Hz}, 3 \mathrm{H}, \mathrm{CH}_{3}\right), 1.22\left(\mathrm{t}, J=7.1 \mathrm{~Hz}, 3 \mathrm{H}, \mathrm{CH}_{3}\right)$.

${ }^{13} \mathrm{C}$ NMR (101 MHz, Acetone- $\left.d_{6}\right) \delta 173.3(\mathrm{C}=\mathrm{O}), 156.2\left(\mathrm{~d}, J=10.8 \mathrm{~Hz}, \mathrm{C}_{\mathrm{Ar}}\right), 146.3\left(\mathrm{C}_{\mathrm{Ar}}\right)$, $135.4\left(\mathrm{C}_{\mathrm{Ar}}\right), 133.1\left(\mathrm{CH}_{\mathrm{Ar}}\right), 123.5\left(\mathrm{CH}_{\mathrm{Ar}}\right), 62.0-61.6\left(\mathrm{~m}, 2 \mathrm{C}, \mathrm{CH}_{2}\right), 37.0(\mathrm{~d}, J=2.3 \mathrm{~Hz}, \mathrm{CH})$, $35.9\left(\mathrm{~d}, J=8.8 \mathrm{~Hz}, \mathrm{CH}_{2}\right), 33.5\left(\mathrm{CH}_{2}\right), 28.0\left(\mathrm{~d}, J=141.5 \mathrm{~Hz}, \mathrm{CH}_{2}\right), 17.0-16.5\left(\mathrm{~m}, 2 \mathrm{C}, \mathrm{CH}_{3}\right)$.

IR (neat), $v\left(\mathrm{~cm}^{-1}\right): 3197,2982,2926,1677,1439,1363,1220,1166,1051,1022,963,831$, 796.

HRMS (ESI) m/z: [M + H] ${ }^{+}$Calcd for $\mathrm{C}_{14} \mathrm{H}_{21} \mathrm{ClN}_{2} \mathrm{O}_{4} \mathrm{P}$ 347.0922; Found 347.0936.

$\mathrm{R}_{\mathrm{f}}=0.32(\mathrm{EtOAc} / \mathrm{MeOH}=10 \%)$. 
2-chloro-9-(3-oxobutyl)-5,7,8,9-tetrahydro-6H-pyrido[3,2-b]azepin-6-one (5aj)<smiles>CC(=O)CCC1CCC(=O)Nc2ccc(Cl)nc21</smiles>

Chemical Formula: $\mathrm{C}_{13} \mathrm{H}_{15} \mathrm{CIN}_{2} \mathrm{O}_{2}$ Molecular Weight: 266.73

Following the general procedure E, the reaction was carried out with the solution of 4aj (286 $\mathrm{mg}, 0.61 \mathrm{mmol}, 1$ eq.) in chlorobenzene $(61 \mathrm{~mL})$ with 5 eq. of DTBP $(0.57 \mathrm{~mL})$ for $4 \mathrm{~h}$. Flash column chromatography on silica gel (eluent: PE/EtOAc $=70 \%$ ) afforded target product 5aj (77 $\mathrm{mg}, 47 \%)$ as a non-crystalline white solid.

${ }^{1} \mathrm{H}$ NMR (400 MHz, Chloroform- $d$ ) $\delta 9.34(\mathrm{~s}, 1 \mathrm{H}, \mathrm{NH}), 7.30\left(\mathrm{~d}, J=8.3 \mathrm{~Hz}, 1 \mathrm{H}, \mathrm{CH}_{\mathrm{Ar}}\right), 7.17$ $\left(\mathrm{dd}, J=8.3 \mathrm{~Hz}, 1 \mathrm{H}, \mathrm{CH}_{\mathrm{Ar}}\right), 3.19-3.03(\mathrm{~m}, 1 \mathrm{H}, \mathrm{CH}), 2.61-2.23\left(\mathrm{~m}, 6 \mathrm{H}, 2 \times \mathrm{CH}_{2}, \mathrm{CH}_{A l} \mathrm{H}\right.$, $\left.\mathrm{CH}_{B 1} \mathrm{H}\right), 2.09\left(\mathrm{~s}, 3 \mathrm{H}, \mathrm{CH}_{3}\right), 1.97-1.78\left(\mathrm{~m}, 2 \mathrm{H}, \mathrm{CH} H_{A 2}\right.$ and $\left.\mathrm{CH}_{B 2}\right)$.

${ }^{13} \mathrm{C}$ NMR (101 MHz, Chloroform- $\left.d\right) \delta 208.8(\mathrm{C}=\mathrm{O}), 175.7(\mathrm{C}=\mathrm{O}), 156.0\left(\mathrm{C}_{\mathrm{Ar}}\right), 146.9\left(\mathrm{C}_{\mathrm{Ar}}\right)$, 133.6 $\left(\mathrm{C}_{\mathrm{Ar}}\right), 131.9\left(\mathrm{CH}_{\mathrm{Ar}}\right), 122.6\left(\mathrm{CH}_{\mathrm{Ar}}\right), 41.7\left(\mathrm{CH}_{2}\right), 40.4(\mathrm{CH}), 34.5\left(\mathrm{CH}_{2}\right), 32.8\left(\mathrm{CH}_{2}\right), 30.0$ $\left(\mathrm{CH}_{3}\right), 25.2\left(\mathrm{CH}_{2}\right)$.

IR (neat), $v\left(\mathrm{~cm}^{-1}\right): 2929,1668,1583,1435,1359,1257,1158,1134,917,831,730$.

HRMS (ESI) m/z: $[\mathrm{M}+\mathrm{H}]^{+}$Calcd for $\mathrm{C}_{13} \mathrm{H}_{16} \mathrm{ClN}_{2} \mathrm{O}_{2}$ 267.0895; Found 267.0898.

$\mathrm{R}_{\mathrm{f}}=0.30(\mathrm{PE} / \mathrm{EtOAc}=70 \%)$.

m.p. $=125-134^{\circ} \mathrm{C}$. 


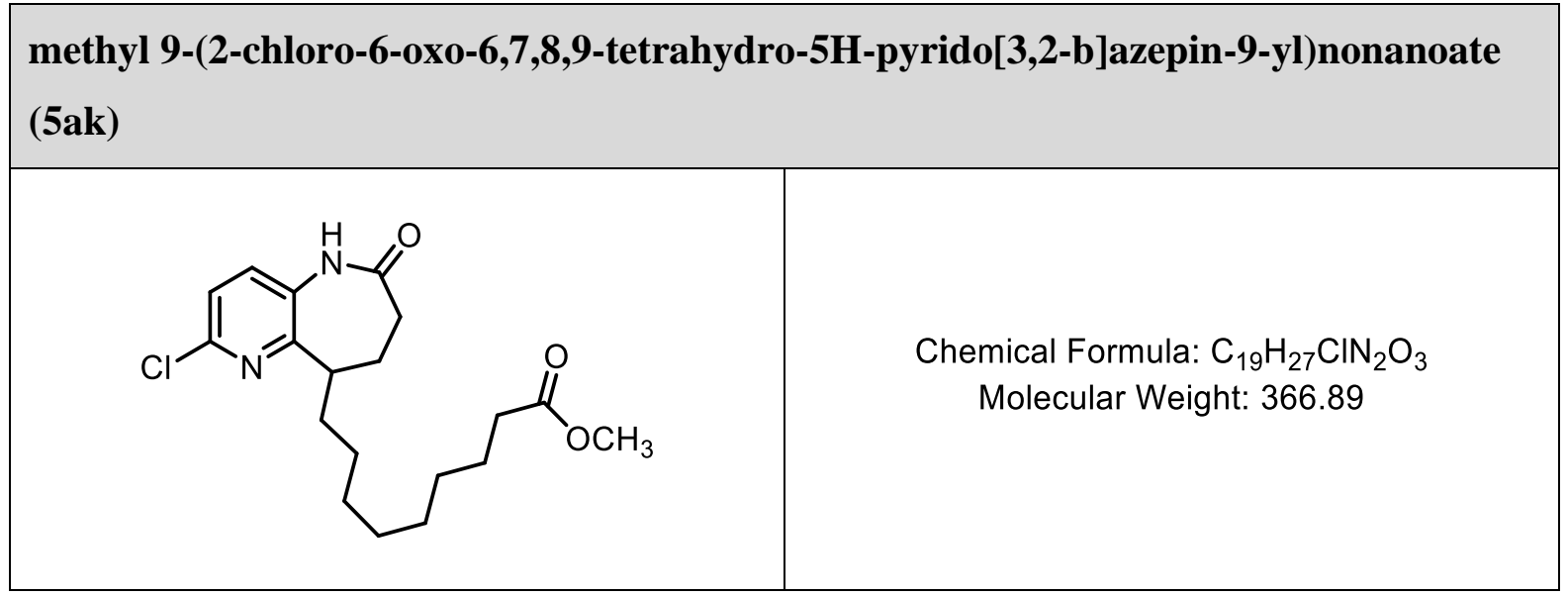

Following the general procedure $\mathrm{E}$, the reaction was carried out with the solution of $\mathbf{4 a k}$ (346 $\mathrm{mg}, 0.64 \mathrm{mmol}, 1$ eq.) in chlorobenzene $(61 \mathrm{~mL})$ with 5 eq. of DTBP $(0.56 \mathrm{~mL})$ for $5 \mathrm{~h}$. Flash column chromatography on silica gel (eluent: PE/EtOAc $=50 \%$ ) afforded target product 5ak (100 mg, 44\%) as a yellow non-crystalline solid.

${ }^{1} \mathrm{H}$ NMR (400 MHz, Chloroform- $d$ ) $\delta 9.05(\mathrm{~s}, 1 \mathrm{H}, \mathrm{NH}), 7.27\left(\mathrm{~d}, J=8.2 \mathrm{~Hz}, 1 \mathrm{H}, \mathrm{CH}_{\mathrm{Ar}}\right), 7.16$ $\left(\mathrm{d}, J=8.2 \mathrm{~Hz}, 1 \mathrm{H}, \mathrm{CH}_{\mathrm{Ar}}\right), 3.63\left(\mathrm{~s}, 3 \mathrm{H}, \mathrm{CH}_{3}\right), 3.16-2.97(\mathrm{~m}, 1 \mathrm{H}, \mathrm{CH}), 2.56-2.41(\mathrm{~m}, 1 \mathrm{H}$, $\left.\mathrm{CH}_{A l} \mathrm{H}\right), 2.38-2.30\left(\mathrm{~m}, 2 \mathrm{H}, \mathrm{CH}_{2}\right), 2.27\left(\mathrm{t}, J=7.5 \mathrm{~Hz}, 2 \mathrm{H}, \mathrm{CH}_{2}\right), 2.09-1.95\left(\mathrm{~m}, 1 \mathrm{H}, \mathrm{CH}_{B l} \mathrm{H}\right)$, $1.93-1.82\left(\mathrm{~m}, 1 \mathrm{H}, \mathrm{CH} H_{A 2}\right), 1.65-1.52\left(\mathrm{~m}, 3 \mathrm{H}, \mathrm{CH}_{2}\right.$ and $\left.\mathrm{CH}_{B 2}\right), 1.34-1.19(\mathrm{~m}, 10 \mathrm{H}, 5 \mathrm{x}$ $\left.\mathrm{CH}_{2}\right)$.

${ }^{13} \mathrm{C}$ NMR (101 MHz, Chloroform- $\left.d\right) \delta 175.9(\mathrm{C}=\mathrm{O}), 174.4(\mathrm{C}=\mathrm{O}), 157.3\left(\mathrm{C}_{\mathrm{Ar}}\right), 147.0\left(\mathrm{C}_{\mathrm{Ar}}\right)$, $133.3\left(\mathrm{C}_{\mathrm{Ar}}\right), 131.7\left(\mathrm{CH}_{\mathrm{Ar}}\right), 122.3\left(\mathrm{CH}_{\mathrm{Ar}}\right), 51.5\left(\mathrm{CH}_{3}\right), 41.5(\mathrm{CH}), 34.2\left(\mathrm{CH}_{2}\right), 34.2\left(\mathrm{CH}_{2}\right), 32.9$ $\left(\mathrm{CH}_{2}\right), 31.4\left(\mathrm{CH}_{2}\right), 29.6\left(\mathrm{CH}_{2}\right), 29.3\left(\mathrm{CH}_{2}\right), 29.2\left(\mathrm{CH}_{2}\right), 29.2\left(\mathrm{CH}_{2}\right), 27.6\left(\mathrm{CH}_{2}\right), 25.0\left(\mathrm{CH}_{2}\right)$.

IR (neat), $v\left(\mathrm{~cm}^{-1}\right): 2926,2854,1733,1671,1435,1361,1254,1160,1134,915,829,729$.

HRMS (ESI) m/z: $[\mathrm{M}+\mathrm{H}]^{+}$Calcd for $\mathrm{C}_{19} \mathrm{H}_{28} \mathrm{ClN}_{2} \mathrm{O}_{3}$ 367.1783; Found 367.1785.

$\mathrm{R}_{\mathrm{f}}=0.30(\mathrm{PE} / \mathrm{EtOAc}=50 \%)$. 


\section{1-((2-chloro-6-oxo-6,7,8,9-tetrahydro-5H-pyrido[3,2-b]azepin-9-yl)methyl)-3,9- dimethyl-3,9-dihydro-1H-purine-2,6-dione (5al)}

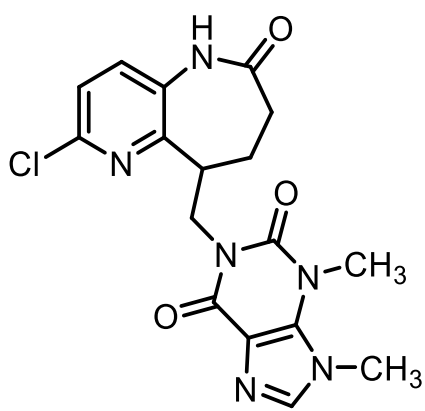

Chemical Formula: $\mathrm{C}_{17} \mathrm{H}_{17} \mathrm{CIN}_{6} \mathrm{O}_{3}$

Molecular Weight: 388.81

Following the general procedure E, the reaction was carried out with the solution of 4al (180 $\mathrm{mg}, 0.31 \mathrm{mmol}, 1$ eq.) in chlorobenzene $(30 \mathrm{~mL})$ with 5 eq. of DTBP $(0.28 \mathrm{~mL})$ for $5.5 \mathrm{~h}$. Recrystallization from EtOAc in freezer afforded target product 5al (48 mg, 40\%) as a yellow solid.

${ }^{1} \mathrm{H}$ NMR (400 MHz, Chloroform- $d$ ) $\delta 8.42$ (s, 1H, NH), 7.53 (d, J=0.7 Hz, 1H, CH), 7.24 (d, $\left.J=8.3 \mathrm{~Hz}, 1 \mathrm{H}, \mathrm{CH}_{\mathrm{Ar}}\right), 7.17\left(\mathrm{~d}, J=8.3 \mathrm{~Hz}, 1 \mathrm{H}, \mathrm{CH}_{\mathrm{Ar}}\right), 4.75\left(\mathrm{dd}, J=13.6,9.8 \mathrm{~Hz}, 1 \mathrm{H}, \mathrm{CH}_{A l} \mathrm{H}\right)$, $4.37\left(\mathrm{dd}, J=13.6,4.2 \mathrm{~Hz}, 1 \mathrm{H}, \mathrm{CH} H_{A 2}\right), 3.97\left(\mathrm{~d}, J=0.6 \mathrm{~Hz}, 3 \mathrm{H}, \mathrm{CH}_{3}\right), 3.67-3.57(\mathrm{~m}, 1 \mathrm{H}$, $\mathrm{CH}), 3.56\left(\mathrm{~s}, 3 \mathrm{H}, \mathrm{CH}_{3}\right), 2.41-2.28\left(\mathrm{~m}, 3 \mathrm{H}, \mathrm{CH}_{2}, \mathrm{CH}_{B 1} \mathrm{H}\right), 2.24-2.08\left(\mathrm{~m}, 1 \mathrm{H}, \mathrm{CH}_{B 2}\right)$.

${ }^{13} \mathrm{C}$ NMR (101 MHz, Chloroform- $\left.d\right) \delta 174.5(\mathrm{C}=\mathrm{O}), 155.5(\mathrm{C}=\mathrm{O}), 155.3\left(\mathrm{C}_{\mathrm{Ar}}\right), 151.8(\mathrm{C}=\mathrm{O})$, $149.0(\mathrm{C}), 146.8\left(\mathrm{C}_{\mathrm{Ar}}\right), 141.8(\mathrm{CH}), 133.1\left(\mathrm{C}_{\mathrm{Ar}}\right), 131.7\left(\mathrm{CH}_{\mathrm{Ar}}\right), 122.8\left(\mathrm{CH}_{\mathrm{Ar}}\right), 107.7(\mathrm{C}), 43.4$ $\left(\mathrm{CH}_{2}\right), 39.5(\mathrm{CH}), 33.8\left(\mathrm{CH}_{3}\right), 32.5\left(\mathrm{CH}_{2}\right), 31.9\left(\mathrm{CH}_{2}\right), 29.9\left(\mathrm{CH}_{3}\right)$.

IR (neat), $v\left(\mathrm{~cm}^{-1}\right): 3249,2939,1702,1657,1444,1362,1231,1138,763,739$.

HRMS (ESI) m/z: [M + H] $]^{+}$Calcd for $\mathrm{C}_{17} \mathrm{H}_{18} \mathrm{ClN}_{6} \mathrm{O}_{3} 389.1123$; Found 389.1129.

$\mathrm{R}_{\mathrm{f}}=0.08(\mathrm{EtOAc} / \mathrm{MeOH}=2 \%)$.

m.p. $=155-165^{\circ} \mathrm{C}$ (with decomposition). 


\section{2-chloro-9-((perfluorophenyl)methyl)-5,7,8,9-tetrahydro-6H-pyrido[3,2-b]azepin-6- one (5am)}<smiles>O=C1CCC(Cc2c(F)c(F)c(F)c(F)c2F)c2nc(Cl)ccc2N1</smiles>

Following the general procedure E, the reaction was carried out with the solution of 4am (274 $\mathrm{mg}, 0.47 \mathrm{mmol}, 1$ eq.) in chlorobenzene $(48 \mathrm{~mL})$ with 5 eq. of DTBP $(0.44 \mathrm{~mL})$ for $3 \mathrm{~h}$. Flash column chromatography on silica gel with (eluent: $\mathrm{PE} / \mathrm{EtOAc}=30 \% \rightarrow 45 \%$ ) afforded target product 5am (139 $\mathrm{mg}, 77 \%)$ as a yellow solid.

${ }^{1} \mathrm{H}$ NMR (400 MHz, Chloroform- $d$ ) $\delta 8.61(\mathrm{~s}, 1 \mathrm{H}, \mathrm{NH}), 7.30\left(\mathrm{~d}, J=8.2 \mathrm{~Hz}, 1 \mathrm{H}, \mathrm{CH}_{\mathrm{Ar}}\right), 7.23$ $\left(\mathrm{d}, J=8.2 \mathrm{~Hz}, 1 \mathrm{H}, \mathrm{CH}_{\mathrm{Ar}}\right), 3.52-3.41(\mathrm{~m}, 1 \mathrm{H}, \mathrm{CH}), 3.37\left(\mathrm{dd}, J=14.1,5.6 \mathrm{~Hz}, 1 \mathrm{H}, \mathrm{C} H_{A l} \mathrm{H}\right)$, $3.21\left(\mathrm{dd}, J=14.1,9.2 \mathrm{~Hz}, 1 \mathrm{H}, \mathrm{CH} H_{A 2}\right), 2.47-2.26\left(\mathrm{~m}, 3 \mathrm{H}, \mathrm{CH}_{2}\right.$ and $\left.\mathrm{CH}_{B 1} \mathrm{H}\right), 2.10-1.96(\mathrm{~m}$, $\left.1 \mathrm{H}, \mathrm{CH} H_{B 2}\right)$.

${ }^{13} \mathrm{C}$ NMR $(101 \mathrm{MHz}$, Chloroform- $d) \delta 174.8(\mathrm{C}=\mathrm{O}), 155.2\left(\mathrm{C}_{\mathrm{Ar}}\right), 147.4\left(\mathrm{C}_{\mathrm{Ar}}\right), 133.0\left(\mathrm{C}_{\mathrm{Ar}}\right)$, $131.9\left(\mathrm{CH}_{\mathrm{Ar}}\right), 123.2\left(\mathrm{CH}_{\mathrm{Ar}}\right), 113.3\left(\mathrm{C}_{\mathrm{Ar}}\right), 40.2(\mathrm{CH}), 33.6\left(\mathrm{CH}_{2}\right), 32.6\left(\mathrm{CH}_{2}\right), 24.9\left(\mathrm{CH}_{2}\right) . \mathrm{C}-\mathrm{F}$ signals are not observed.

IR (neat), $v\left(\mathrm{~cm}^{-1}\right)$ : 2938, 1675, 1520, 1500, 1439, 1368, 1190, 1124, 1049, 962, 739.

HRMS (ESI) m/z: [M + H] ${ }^{+}$Calcd for $\mathrm{C}_{16} \mathrm{H}_{11} \mathrm{ClF}_{5} \mathrm{~N}_{2} \mathrm{O}$ 377.0475; Found 377.0480.

$\mathrm{R}_{\mathrm{f}}=0.43(\mathrm{PE} / \mathrm{EtOAc}=50 \%)$.

m.p. $=146-150^{\circ} \mathrm{C}$. 


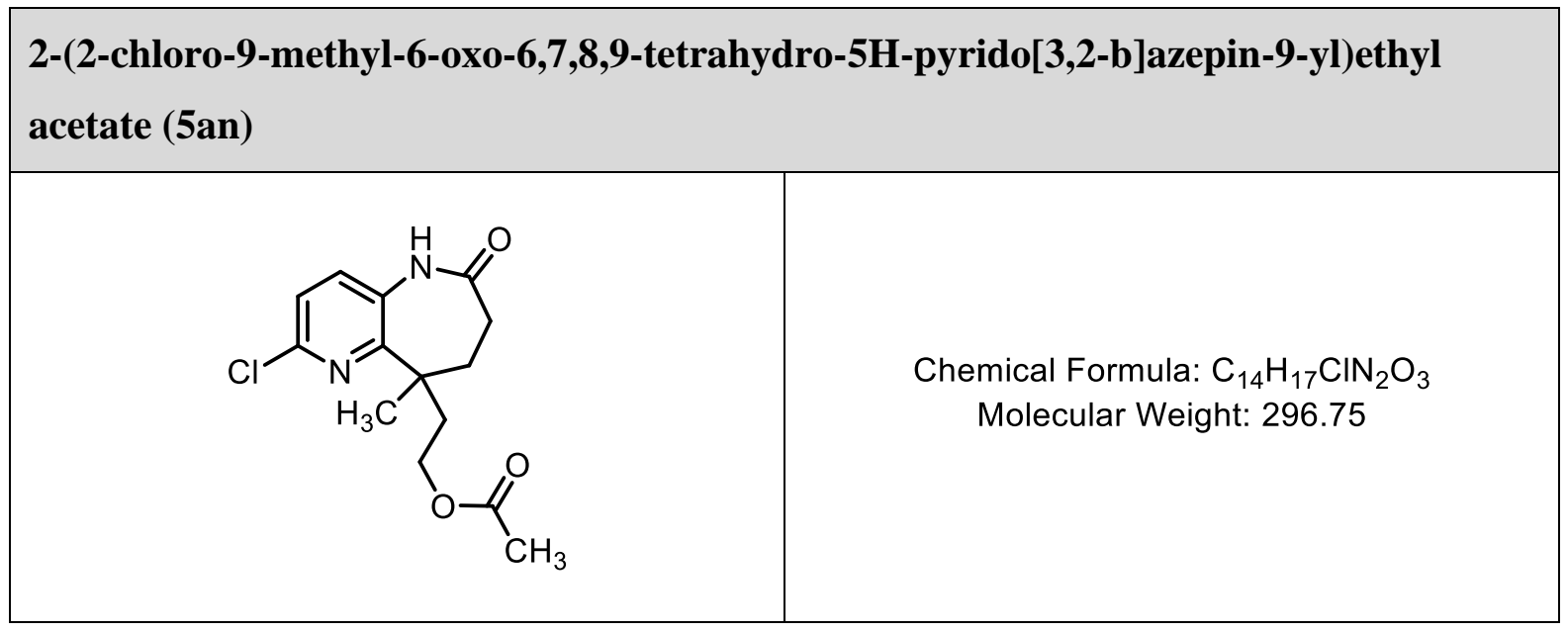

Following the general procedure E, the reaction was carried out with the solution of 4an (275 $\mathrm{mg}, 0.55 \mathrm{mmol}, 1$ eq.) in chlorobenzene $(55 \mathrm{~mL})$ with 5 eq. of DTBP $(0.51 \mathrm{~mL})$ for $3 \mathrm{~h}$. Flash column chromatography on silica gel with (eluent: PE/EtOAc $=0 \% \rightarrow 50 \% \rightarrow 70 \%$ ) afforded target product 5an (75 mg, 46\%) as a yellow non-crystalline solid.

${ }^{1} \mathrm{H}$ NMR (400 MHz, Chloroform- $d$ ) $\delta 9.28$ (s, 1H, NH), 7.29 (d, J = 8.2 Hz, 1H, CH $\mathrm{Cr}_{\mathrm{Ar}}$ ), 7.13 $\left(\mathrm{d}, J=8.2 \mathrm{~Hz}, 1 \mathrm{H}, \mathrm{CH}_{\mathrm{Ar}}\right), 4.07-3.88\left(\mathrm{~m}, 2 \mathrm{H}, \mathrm{CH}_{2}\right), 2.61-2.45\left(\mathrm{~m}, 2 \mathrm{H}, \mathrm{CH}_{2}\right), 2.36-2.10(\mathrm{~m}$, $\left.3 \mathrm{H}, \mathrm{CH}_{2}, \mathrm{CH}_{A l} \mathrm{H}\right), 2.09-1.99\left(\mathrm{~m}, 1 \mathrm{H}, \mathrm{CH} H_{A 2}\right), 1.91\left(\mathrm{~s}, 3 \mathrm{H}, \mathrm{CH}_{3}\right), 1.45\left(\mathrm{~s}, 3 \mathrm{H}, \mathrm{CH}_{3}\right)$.

${ }^{13} \mathrm{C}$ NMR (101 MHz, Chloroform- $\left.d\right) \delta 176.4(\mathrm{C}=\mathrm{O}), 171.0(\mathrm{C}=\mathrm{O}), 155.4\left(\mathrm{C}_{\mathrm{Ar}}\right), 145.2\left(\mathrm{C}_{\mathrm{Ar}}\right)$, $132.9\left(\mathrm{CH}_{\mathrm{Ar}}\right), 132.4\left(\mathrm{C}_{\mathrm{Ar}}\right), 122.9\left(\mathrm{CH}_{\mathrm{Ar}}\right), 61.4\left(\mathrm{CH}_{2}\right), 43.4(\mathrm{C}), 40.0\left(\mathrm{CH}_{2}\right), 36.5\left(\mathrm{CH}_{2}\right), 32.1$ $\left(\mathrm{CH}_{2}\right), 28.3\left(\mathrm{CH}_{3}\right), 21.0\left(\mathrm{CH}_{3}\right)$.

IR (neat), $v\left(\mathrm{~cm}^{-1}\right): 2965,2932,1733,1673,1440,1369,1237,1149,1034,913,833$.

HRMS (ESI) m/z: [M + H] ${ }^{+}$Calcd for $\mathrm{C}_{14} \mathrm{H}_{18} \mathrm{ClN}_{2} \mathrm{O}_{3}$ 297.1000; Found 297.0997.

$\mathrm{R}_{\mathrm{f}}=0.29(\mathrm{PE} / \mathrm{EtOAc}=50 \%)$. 
<smiles>CC(=O)OCC1CCC(=O)Nc2ccc(Br)nc21</smiles>

Chemical Formula: $\mathrm{C}_{12} \mathrm{H}_{13} \mathrm{BrN}_{2} \mathrm{O}_{3}$ Molecular Weight: 313.15

Following the general procedure E, the reaction was carried out with the solution of $\mathbf{4 b a}(270$ $\mathrm{mg}, 0.53 \mathrm{mmol}, 1$ eq.) in chlorobenzene $(52 \mathrm{~mL})$ with 5 eq. of DTBP $(0.49 \mathrm{~mL})$ for $5 \mathrm{~h}$. Flash column chromatography on silica gel (eluent: $\mathrm{PE} / \mathrm{EtOAc}=40 \% \rightarrow 70 \%$ ) afforded target product $\mathbf{5 b a}(91 \mathrm{mg}, 55 \%)$ as a white solid.

${ }^{1} \mathrm{H}$ NMR (400 MHz, Chloroform- $d$ ) $\delta 8.73(\mathrm{~s}, 1 \mathrm{H}, \mathrm{NH}), 7.38$ (d, $J=8.2 \mathrm{~Hz}, 1 \mathrm{H}, \mathrm{CH}_{\mathrm{Ar}}$ ), 7.20 $\left(\mathrm{d}, J=8.2 \mathrm{~Hz}, 1 \mathrm{H}, \mathrm{CH}_{\mathrm{Ar}}\right), 4.72\left(\mathrm{dd}, J=11.2,6.2 \mathrm{~Hz}, 1 \mathrm{H}, \mathrm{CH}_{A l} \mathrm{H}\right), 4.39(\mathrm{dd}, J=11.2,7.5 \mathrm{~Hz}$, $\left.1 \mathrm{H}, \mathrm{CH} H_{A 2}\right), 3.54-3.43(\mathrm{~m}, 1 \mathrm{H}, \mathrm{CH}), 2.64-2.51\left(\mathrm{~m}, 1 \mathrm{H}, \mathrm{CH}_{B I} \mathrm{H}\right), 2.44-2.34\left(\mathrm{~m}, 2 \mathrm{H}, \mathrm{CH}_{2}\right)$, $2.04\left(\mathrm{~s}, 3 \mathrm{H}, \mathrm{CH}_{3}\right), 2.02-1.94\left(\mathrm{~m}, 1 \mathrm{H}, \mathrm{CH} H_{B 2}\right)$.

${ }^{13} \mathrm{C}$ NMR (101 MHz, Chloroform- $\left.d\right) \delta 174.9(\mathrm{C}=\mathrm{O}), 171.1(\mathrm{C}=\mathrm{O}), 154.7\left(\mathrm{C}_{\mathrm{Ar}}\right), 137.4\left(\mathrm{C}_{\mathrm{Ar}}\right)$, 133.6 $\left(\mathrm{C}_{\mathrm{Ar}}\right), 131.6\left(\mathrm{CH}_{\mathrm{Ar}}\right), 126.9\left(\mathrm{CH}_{\mathrm{Ar}}\right), 64.9\left(\mathrm{CH}_{2}\right), 40.3(\mathrm{CH}), 32.5\left(\mathrm{CH}_{2}\right), 31.3\left(\mathrm{CH}_{2}\right), 21.0$ $\left(\mathrm{CH}_{3}\right)$.

IR (neat), $v\left(\mathrm{~cm}^{-1}\right): 3220,2940,1737,1675,1438,1369,1240,1040,832,760$.

HRMS (ESI) m/z: $[\mathrm{M}+\mathrm{H}]^{+}$Calcd for $\mathrm{C}_{12} \mathrm{H}_{14} \mathrm{BrN}_{2} \mathrm{O}_{3}$ 313.0182; Found 313.0186.

$\mathrm{R}_{\mathrm{f}}=0.18(\mathrm{PE} / \mathrm{EtOAc}=50 \%)$.

m.p. $=186-187^{\circ} \mathrm{C}($ crystallized from PE/EtOAc). 


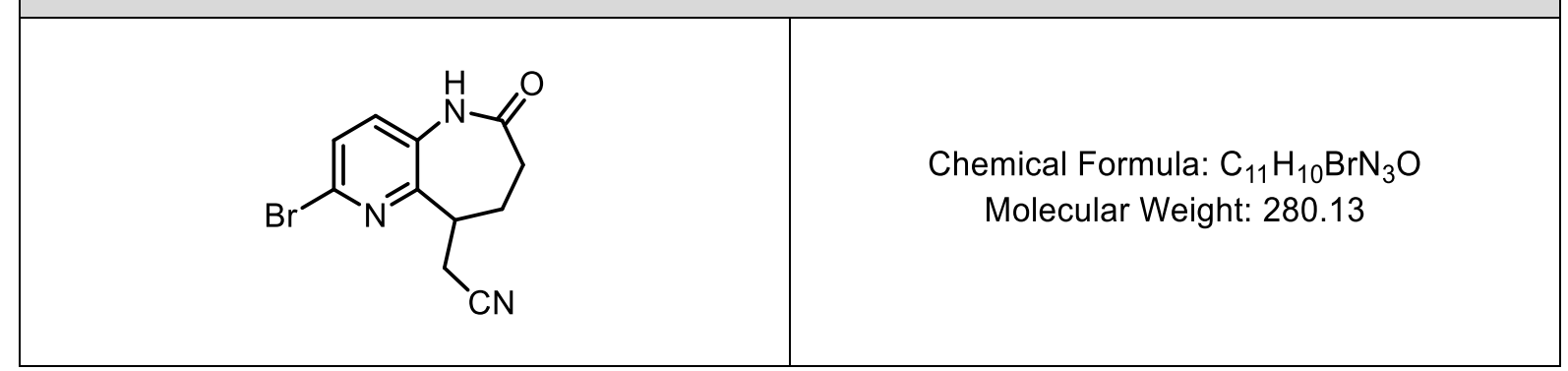

Following the general procedure E, the reaction was carried out with the solution of $\mathbf{4 b b}$ (231 $\mathrm{mg}, 0.48 \mathrm{mmol}, 1$ eq.) in chlorobenzene ( $48 \mathrm{~mL})$ with 5 eq. of DTBP $(0.45 \mathrm{~mL})$ for $4.5 \mathrm{~h}$. Flash column chromatography on silica gel (eluent: PE/EtOAc $=70 \%$ ) afforded target product $\mathbf{5 b b}$ $(65 \mathrm{mg}, 48 \%)$ as a white solid.

${ }^{1} \mathrm{H}$ NMR $\left(400 \mathrm{MHz}, \mathrm{DMSO}-d_{6}\right) \delta 9.79(\mathrm{~s}, 1 \mathrm{H}, \mathrm{NH}), 7.58\left(\mathrm{dd}, J=8.3 \mathrm{~Hz}, 1 \mathrm{H}, \mathrm{CH}_{\mathrm{Ar}}\right), 7.33(\mathrm{~d}$, $\left.J=8.3 \mathrm{~Hz}, 1 \mathrm{H}, \mathrm{CH}_{\mathrm{Ar}}\right), 3.47-3.36(\mathrm{~m}, 1 \mathrm{H}, \mathrm{CH}), 2.95\left(\mathrm{dd}, J=16.6,7.5 \mathrm{~Hz}, 1 \mathrm{H}, \mathrm{CH}_{A l} \mathrm{H}\right), 2.86$ $\left(\mathrm{dd}, J=16.6,6.0 \mathrm{~Hz}, 1 \mathrm{H}, \mathrm{CH} H_{A 2}\right), 2.48-2.40\left(\mathrm{~m}, 1 \mathrm{H}, \mathrm{CH}_{B 1} \mathrm{H}\right), 2.38-2.26\left(\mathrm{~m}, 1 \mathrm{H}, \mathrm{C} H_{C l} \mathrm{H}\right)$, $2.23-2.13\left(\mathrm{~m}, 1 \mathrm{H}, \mathrm{CH} H_{C 2}\right), 1.99-1.85\left(\mathrm{~m}, 1 \mathrm{H}, \mathrm{CH} H_{B 2}\right)$.

${ }^{13} \mathrm{C}$ NMR $\left(101 \mathrm{MHz}, \mathrm{DMSO}-d_{6}\right) \delta 172.4(\mathrm{C}=\mathrm{O}), 153.5\left(\mathrm{C}_{\mathrm{Ar}}\right), 135.0\left(\mathrm{C}_{\mathrm{Ar}}\right), 134.8\left(\mathrm{C}_{\mathrm{Ar}}\right), 132.2$ $\left(\mathrm{CH}_{\mathrm{Ar}}\right), 127.0\left(\mathrm{CH}_{\mathrm{Ar}}\right), 119.6(\mathrm{CN}), 37.3(\mathrm{CH}), 32.8\left(\mathrm{CH}_{2}\right), 32.4\left(\mathrm{CH}_{2}\right), 19.1\left(\mathrm{CH}_{2}\right)$.

IR (neat), $v\left(\mathrm{~cm}^{-1}\right): 1666,1436,1374,1330,1181,1156,1130,1079,920,831,752,631,536$. HRMS (ESI) m/z: $[\mathrm{M}+\mathrm{H}]^{+}$Calcd for $\mathrm{C}_{11} \mathrm{H}_{11} \mathrm{BrN}_{3} \mathrm{O} 280.0080$; Found 280.0083.

$\mathrm{R}_{\mathrm{f}}=0.32(\mathrm{PE} / \mathrm{EtOAc}=70 \%)$.

m.p. $=206-209^{\circ} \mathrm{C}$ (with decomposition). 


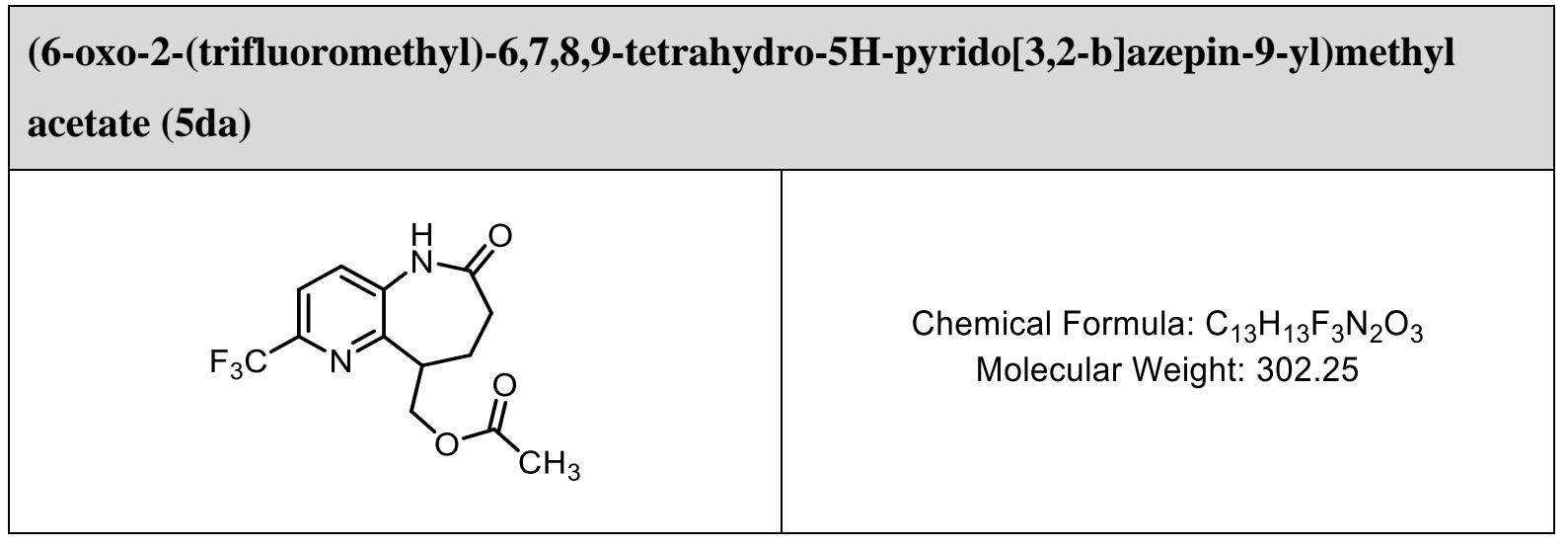

Following the general procedure $\mathrm{E}$, the reaction was carried out with the solution of 4da (182 $\mathrm{mg}, 0.36 \mathrm{mmol}, 1$ eq.) in chlorobenzene $(36 \mathrm{~mL})$ with 5 eq. of DTBP $(0.34 \mathrm{~mL})$ for $4 \mathrm{~h}$. Flash column chromatography on silica gel (eluent: PE/EtOAc $=50 \% \rightarrow 60 \%$ ) afforded target product $\mathbf{5 d a}(61 \mathrm{mg}, 56 \%)$ as a white solid.

${ }^{1} \mathrm{H}$ NMR (400 MHz, Chloroform- $d$ ) $\delta 8.86(\mathrm{~s}, 1 \mathrm{H}, \mathrm{NH}), 7.60\left(\mathrm{~d}, J=8.2 \mathrm{~Hz}, 1 \mathrm{H}, \mathrm{CH}_{\mathrm{Ar}}\right), 7.47$ $\left(\mathrm{d}, J=8.2 \mathrm{~Hz}, 1 \mathrm{H}, \mathrm{CH}_{\mathrm{Ar}}\right), 4.80\left(\mathrm{dd}, J=11.2,6.5 \mathrm{~Hz}, 1 \mathrm{H}, \mathrm{CH}_{A l} \mathrm{H}\right), 4.42(\mathrm{dd}, J=11.2,7.1 \mathrm{~Hz}$, $\left.1 \mathrm{H}, \mathrm{CH} H_{A 2}\right), 3.65-3.51(\mathrm{~m}, 1 \mathrm{H}, \mathrm{CH}), 2.68-2.53\left(\mathrm{~m}, 1 \mathrm{H}, \mathrm{CH}_{B 1} \mathrm{H}\right), 2.53-2.36\left(\mathrm{~m}, 2 \mathrm{H}, \mathrm{CH}_{2}\right)$, $2.10-2.03\left(\mathrm{~m}, 1 \mathrm{H}, \mathrm{CH} H_{B 2}\right), 2.02\left(\mathrm{~s}, 3 \mathrm{H}, \mathrm{CH}_{3}\right)$.

${ }^{13} \mathrm{C}$ NMR (101 MHz, Chloroform- $\left.d\right) \delta 174.8(\mathrm{C}=\mathrm{O}), 171.1(\mathrm{C}=\mathrm{O}), 154.2\left(\mathrm{C}_{\mathrm{Ar}}\right), 144.4(\mathrm{C}-\mathrm{F}$, $\left.{ }^{2} J_{C-F}=35.3 \mathrm{~Hz}, \mathrm{C}_{\mathrm{Ar}}\right), 136.6\left(\mathrm{C}_{\mathrm{Ar}}\right), 129.5\left(\mathrm{CH}_{\mathrm{Ar}}\right), 121.4\left(\mathrm{C}-\mathrm{F},{ }^{1} J_{C-F}=273.7 \mathrm{~Hz}, \mathrm{CF}_{3}\right), 119.6(\mathrm{C}-$ $\left.\mathrm{F},{ }^{3} J_{C-F}=2.4 \mathrm{~Hz}, \mathrm{CH}_{\mathrm{Ar}}\right), 64.7\left(\mathrm{CH}_{2}\right), 40.5(\mathrm{CH}), 32.6\left(\mathrm{CH}_{2}\right), 30.9\left(\mathrm{CH}_{2}\right), 20.9\left(\mathrm{CH}_{3}\right)$.

IR (neat), $v\left(\mathrm{~cm}^{-1}\right): 1737,1678,1586,1471,1368,1337,1237,1133,1115,1042,848,737$.

HRMS (ESI) m/z: $[\mathrm{M}+\mathrm{H}]^{+}$Calcd for $\mathrm{C}_{13} \mathrm{H}_{14} \mathrm{~F}_{3} \mathrm{~N}_{2} \mathrm{O}_{3}$ 303.0951; Found 303.0954.

$\mathrm{R}_{\mathrm{f}}=0.27(\mathrm{PE} / \mathrm{EtOAc}=50 \%)$.

m.p. $=164-167^{\circ} \mathrm{C}($ crystallized from PE/EtOAc). 
2-(6-oxo-2-(trifluoromethyl)-6,7,8,9-tetrahydro-5H-pyrido[3,2-b]azepin-9yl)acetonitrile $(5 \mathrm{db})$

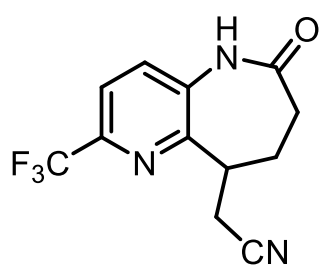

Chemical Formula: $\mathrm{C}_{12} \mathrm{H}_{10} \mathrm{~F}_{3} \mathrm{~N}_{3} \mathrm{O}$

Molecular Weight: 269.23

Following the general procedure E, the reaction was carried out with the solution of $\mathbf{4 d b}$ (262 $\mathrm{mg}, 0.56 \mathrm{mmol}, 1$ eq.) in chlorobenzene $(56 \mathrm{~mL})$ with 5 eq. of DTBP $(0.52 \mathrm{~mL})$ for $4 \mathrm{~h}$. Flash column chromatography on silica gel (eluent: PE/EtOAc $=70 \%$ ) afforded target product $\mathbf{5} \mathbf{d b}$ (69 $\mathrm{mg}, 46 \%)$ as a white solid.

${ }^{1} \mathrm{H}$ NMR (400 MHz, Chloroform- $d$ ) $\delta 9.15(\mathrm{~s}, 1 \mathrm{H}, \mathrm{NH}), 7.66\left(\mathrm{~d}, J=8.2 \mathrm{~Hz}, 1 \mathrm{H}, \mathrm{CH}_{\mathrm{Ar}}\right), 7.53$ $\left(\mathrm{d}, J=8.2 \mathrm{~Hz}, 1 \mathrm{H}, \mathrm{CH}_{\mathrm{Ar}}\right), 3.70-3.57(\mathrm{~m}, 1 \mathrm{H}, \mathrm{CH}), 3.07\left(\mathrm{dd}, J=16.9,6.5 \mathrm{~Hz}, 1 \mathrm{H}, \mathrm{CH}_{A l} \mathrm{H}\right)$, $2.90\left(\mathrm{dd}, J=16.9,7.5 \mathrm{~Hz}, 1 \mathrm{H}, \mathrm{CH} H_{A 2}\right), 2.83-2.69\left(\mathrm{~m}, 1 \mathrm{H}, \mathrm{CH}_{B 1} \mathrm{H}\right), 2.55-2.38\left(\mathrm{~m}, 2 \mathrm{H}, \mathrm{CH}_{2}\right)$, $2.15-2.03\left(\mathrm{~m}, 1 \mathrm{H}, \mathrm{CH} H_{B 2}\right)$.

${ }^{13} \mathrm{C}$ NMR (101 MHz, Chloroform- $\left.d\right) \delta 174.5(\mathrm{C}=\mathrm{O}), 152.8\left(\mathrm{C}_{\mathrm{Ar}}\right), 144.4\left(\mathrm{C}-\mathrm{F},{ }^{2} J_{C-F}=35.6 \mathrm{~Hz}\right.$, $\left.\mathrm{C}_{\mathrm{Ar}}\right), 136.6\left(\mathrm{C}_{\mathrm{Ar}}\right), 129.9\left(\mathrm{CH}_{\mathrm{Ar}}\right), 121.3\left(\mathrm{C}-\mathrm{F},{ }^{1} J_{C-F}=273.8 \mathrm{~Hz}, \mathrm{CF}_{3}\right), 120.3\left(\mathrm{C}-\mathrm{F},{ }^{3} J_{C-F}=2.8 \mathrm{~Hz}\right.$, $\left.\mathrm{CH}_{\mathrm{Ar}}\right), 118.6(\mathrm{CN}), 38.2(\mathrm{CH}), 33.4\left(\mathrm{CH}_{2}\right), 32.6\left(\mathrm{CH}_{2}\right), 20.0\left(\mathrm{CH}_{2}\right)$.

IR (neat), $v\left(\mathrm{~cm}^{-1}\right): 1678,1588,1472,1371,1336,1263,1178,1132,1110,932,735$.

HRMS (ESI) m/z: [M + H $]^{+}$Calcd for $\mathrm{C}_{12} \mathrm{H}_{11} \mathrm{~F}_{3} \mathrm{~N}_{3} \mathrm{O} 270.0849$; Found 270.0850.

$\mathrm{R}_{\mathrm{f}}=0.30(\mathrm{PE} / \mathrm{EtOAc}=70 \%)$.

m.p. $=176-178^{\circ} \mathrm{C}($ crystallized from PE/EtOAc $)$. 


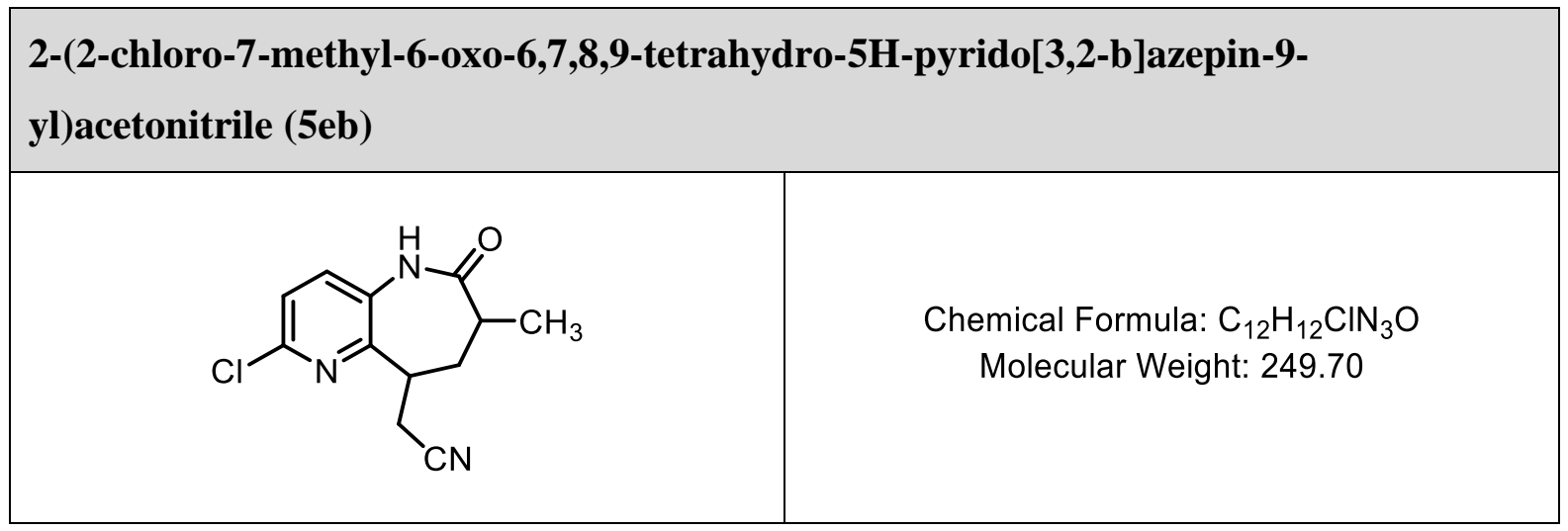

Following the general procedure $\mathrm{E}$, the reaction was carried out with the solution of 4eb (145 $\mathrm{mg}, 0.32 \mathrm{mmol}, 1$ eq.) in chlorobenzene $(32 \mathrm{~mL})$ with 5 eq. of DTBP $(0.30 \mathrm{~mL})$ for $4.5 \mathrm{~h}$. Flash column chromatography on silica gel (eluent: PE/EtOAc $=50 \%$ ) afforded target product 5eb $(37 \mathrm{mg}, 46 \%)$ as a white non-crystalline solid. Mixture of two diastereomers (d.r. $=2.8: 1)$.

Isomer 1:

${ }^{1} \mathrm{H}$ NMR (400 MHz, Chloroform- $d$ ) $\delta 9.11(\mathrm{~s}, 1 \mathrm{H}, \mathrm{NH}), 7.36\left(\mathrm{~d}, J=8.5 \mathrm{~Hz}, 1 \mathrm{H}, \mathrm{CH}_{\mathrm{Ar}}\right), 7.26$ $\left(\mathrm{d}, J=8.5 \mathrm{~Hz}, 1 \mathrm{H}, \mathrm{CH}_{\mathrm{Ar}}\right), 3.66-3.55(\mathrm{~m}, 1 \mathrm{H}, \mathrm{CH}), 2.89-2.84\left(\mathrm{~m}, 2 \mathrm{H}, \mathrm{CH}_{2}\right), 2.77-2.64(\mathrm{~m}$, $1 \mathrm{H}, \mathrm{CH}), 2.59-2.50\left(\mathrm{~m}, 1 \mathrm{H}, \mathrm{CH}_{A l} \mathrm{H}\right), 2.05-1.92\left(\mathrm{~m}, 1 \mathrm{H}, \mathrm{CH} H_{A 2}\right), 1.13(\mathrm{~d}, J=6.9 \mathrm{~Hz}, 3 \mathrm{H}$, $\mathrm{CH}_{3}$ ).

${ }^{13} \mathrm{C}$ NMR (101 MHz, Chloroform- $\left.d\right) \delta 177.4(\mathrm{C}=\mathrm{O}), 151.5\left(\mathrm{C}_{\mathrm{Ar}}\right), 146.3\left(\mathrm{C}_{\mathrm{Ar}}\right), 132.9\left(\mathrm{CH}_{\mathrm{Ar}}\right)$, $132.6\left(\mathrm{C}_{\mathrm{Ar}}\right), 124.1\left(\mathrm{CH}_{\mathrm{Ar}}\right), 118.1(\mathrm{CN}), 41.3(\mathrm{CH}), 39.0\left(\mathrm{CH}_{2}\right), 37.0(\mathrm{CH}), 22.5\left(\mathrm{CH}_{2}\right), 17.0$ $\left(\mathrm{CH}_{3}\right)$.

Isomer 2:

${ }^{1} \mathrm{H}$ NMR (400 MHz, Chloroform- $d$ ) $\delta 8.97$ (s, 1H, NH), 7.35 (d, $J=8.3 \mathrm{~Hz}, 1 \mathrm{H}, \mathrm{CH}_{\mathrm{Ar}}$ ), 7.27 $\left(\mathrm{d}, J=8.3 \mathrm{~Hz}, 1 \mathrm{H}, \mathrm{CH}_{\mathrm{Ar}}\right), 3.58-3.48(\mathrm{~m}, 1 \mathrm{H}, \mathrm{CH}), 3.02-2.90\left(\mathrm{~m}, 1 \mathrm{H}, \mathrm{CH}_{A l} \mathrm{H}\right), 2.84-2.78$ $\left(\mathrm{m}, 1 \mathrm{H}, \mathrm{CH}_{A 2}\right), 2.51-2.43(\mathrm{~m}, 1 \mathrm{H}, \mathrm{CH}), 2.39-2.27\left(\mathrm{~m}, 1 \mathrm{H}, \mathrm{CH}_{B 1} \mathrm{H}\right), 2.16-2.07(\mathrm{~m}, 1 \mathrm{H}$, $\left.\mathrm{CH} H_{B 2}\right), 1.12\left(\mathrm{~d}, J=6.4 \mathrm{~Hz}, 3 \mathrm{H}, \mathrm{CH}_{3}\right)$.

${ }^{13} \mathrm{C}$ NMR (101 MHz, Chloroform- $\left.d\right) \delta 176.6(\mathrm{C}=\mathrm{O}), 153.4\left(\mathrm{C}_{\mathrm{Ar}}\right), 147.3\left(\mathrm{C}_{\mathrm{Ar}}\right), 132.6\left(\mathrm{C}_{\mathrm{Ar}}\right)$, $132.3\left(\mathrm{CH}_{\mathrm{Ar}}\right), 123.6\left(\mathrm{CH}_{\mathrm{Ar}}\right), 118.8(\mathrm{CN}), 43.3\left(\mathrm{CH}_{2}\right), 37.3(\mathrm{CH}), 35.4(\mathrm{CH}), 19.8\left(\mathrm{CH}_{2}\right), 15.4$ $\left(\mathrm{CH}_{3}\right)$.

IR (neat), $v\left(\mathrm{~cm}^{-1}\right): 2933,1671,1580,1443,1394,1273,1137,915,834,734$.

HRMS (ESI) m/z: [M + H $]^{+}$Calcd for $\mathrm{C}_{12} \mathrm{H}_{13} \mathrm{ClN}_{3} \mathrm{O} 250.0742$; Found 250.0743. 
$\mathrm{R}_{\mathrm{f}}=0.53(\mathrm{PE} / \mathrm{EtOAc}=70 \%)$.

m.p. $=130-132^{\circ} \mathrm{C}$. 
(8-chloro-2-oxo-2,3,4,5-tetrahydro-1H-pyrido[4,3-b]azepin-5-yl)methyl acetate (5fa)<smiles>CC(=O)OCC1CCC(=O)Nc2cc(Cl)ncc21</smiles>

Chemical Formula: $\mathrm{C}_{12} \mathrm{H}_{13} \mathrm{CIN}_{2} \mathrm{O}_{3}$ Molecular Weight: 268.70

Following the general procedure E, the reaction was carried out with the solution of $\mathbf{4 f a}$ (236 $\mathrm{mg}, 0.50 \mathrm{mmol}, 1$ eq. $)$ in chlorobenzene $(50 \mathrm{~mL})$ with 5 eq. of DTBP $(0.46 \mathrm{~mL})$ for $3 \mathrm{~h}$. Flash column chromatography on silica gel (eluent: $\mathrm{DCM} / \mathrm{MeOH}=4 \%$ ), followed by the recrystallization from EtOAc, afforded target product $\mathbf{5 f a}(75 \mathrm{mg}, 55 \%)$ as a white solid.

${ }^{1} \mathrm{H}$ NMR (400 MHz, Chloroform- $d$ ) $\delta 8.74(\mathrm{~s}, 1 \mathrm{H}, \mathrm{NH}), 8.20\left(\mathrm{~d}, J=5.3 \mathrm{~Hz}, 1 \mathrm{H}, \mathrm{CH}_{\mathrm{Ar}}\right), 6.86$ (d, J = 5.3 Hz, 1H, $\left.\mathrm{CH}_{\mathrm{Ar}}\right), 4.39-4.25\left(\mathrm{~m}, 2 \mathrm{H}, \mathrm{CH}_{2}\right), 4.14-4.03(\mathrm{~m}, 1 \mathrm{H}, \mathrm{CH}), 2.67-2.49$ (m, $\left.2 \mathrm{H}, \mathrm{CH}_{2}\right), 2.46-2.36\left(\mathrm{~m}, 1 \mathrm{H}, \mathrm{CH}_{A l} \mathrm{H}\right), 2.29-2.16\left(\mathrm{~m}, 1 \mathrm{H}, \mathrm{CH} H_{A 2}\right), 1.96\left(\mathrm{~s}, 3 \mathrm{H}, \mathrm{CH}_{3}\right)$.

${ }^{13} \mathrm{C}$ NMR (101 MHz, Chloroform- $\left.d\right) \delta 175.0(\mathrm{C}=\mathrm{O}), 170.8(\mathrm{C}=\mathrm{O}), 154.0\left(\mathrm{C}_{\mathrm{Ar}}\right), 148.6\left(\mathrm{CH}_{\mathrm{Ar}}\right)$, 147.2 $\left(\mathrm{C}_{\mathrm{Ar}}\right), 125.4\left(\mathrm{C}_{\mathrm{Ar}}\right), 116.2\left(\mathrm{CH}_{\mathrm{Ar}}\right), 64.4\left(\mathrm{CH}_{2}\right), 37.5(\mathrm{CH}), 32.5\left(\mathrm{CH}_{2}\right), 27.2\left(\mathrm{CH}_{2}\right), 20.9$ $\left(\mathrm{CH}_{3}\right)$.

IR (neat), $v\left(\mathrm{~cm}^{-1}\right): 1738,1678,1575,1464,1368,1228,1034,842,733,659,603$.

HRMS (ESI) m/z: [M + H] ${ }^{+}$Calcd for $\mathrm{C}_{12} \mathrm{H}_{14} \mathrm{ClN}_{2} \mathrm{O}_{3}$ 269.0687; Found 269.0689.

$\mathrm{R}_{\mathrm{f}}=0.31(\mathrm{PE} / \mathrm{EtOAc}=70 \%)$.

m.p. $=168-172^{\circ} \mathrm{C}($ crystallized from EtOAc $)$. 
2-((8-chloro-2-oxo-2,3,4,5-tetrahydro-1H-pyrido[4,3-b]azepin-5-yl)methyl)isoindoline1,3-dione (5fc)

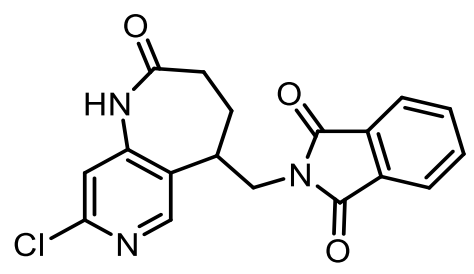

Chemical Formula: $\mathrm{C}_{18} \mathrm{H}_{14} \mathrm{CIN}_{3} \mathrm{O}_{3}$

Molecular Weight: 355.78

Following the general procedure E, the reaction was carried out with the solution of $\mathbf{4 f c}$ (417 $\mathrm{mg}, 0.75 \mathrm{mmol}, 1$ eq.) in chlorobenzene $(75 \mathrm{~mL})$ with 5 eq. of DTBP $(0.69 \mathrm{~mL})$ for $4 \mathrm{~h}$. Flash column chromatography on silica gel (eluent: $\mathrm{PE} / \mathrm{EtOAc}=45 \% \rightarrow 75 \%$ ) afforded target product $\mathbf{5 f c}(151 \mathrm{mg}, 57 \%)$ as a white solid.

${ }^{1} \mathrm{H}$ NMR (400 MHz, DMSO- $\left.d_{6}\right) \delta 10.35(\mathrm{~s}, 1 \mathrm{H}, \mathrm{NH}), 8.07\left(\mathrm{~d}, J=5.3 \mathrm{~Hz}, 1 \mathrm{H}, \mathrm{CH}_{\mathrm{Ar}}\right), 7.85-$ $7.73\left(\mathrm{~m}, 4 \mathrm{H}, \mathrm{CH}_{\mathrm{Phth}}\right), 6.99\left(\mathrm{~d}, J=5.3 \mathrm{~Hz}, 1 \mathrm{H}, \mathrm{CH}_{\mathrm{Ar}}\right), 4.08-3.94\left(\mathrm{~m}, 2 \mathrm{H}, \mathrm{CH}\right.$ and $\left.\mathrm{CH}_{A l} \mathrm{H}\right), 3.82$ $-3.69\left(\mathrm{~m}, 1 \mathrm{H}, \mathrm{CH} H_{A 2}\right), 2.56-2.45\left(\mathrm{~m}, 1 \mathrm{H}, \mathrm{CH}_{B l} \mathrm{H}\right), 2.44-2.35\left(\mathrm{~m}, 1 \mathrm{H}, \mathrm{C} H_{C l} \mathrm{H}\right), 2.34-2.25$ $\left(\mathrm{m}, 1 \mathrm{H}, \mathrm{CH} H_{B 2}\right), 2.23-2.10\left(\mathrm{~m}, 1 \mathrm{H}, \mathrm{CH} H_{C 2}\right)$.

${ }^{13} \mathrm{C}$ NMR $\left(101 \mathrm{MHz}, \mathrm{DMSO}-d_{6}\right) \delta 173.5(\mathrm{C}=\mathrm{O}), 167.4(\mathrm{C}=\mathrm{O}), 152.3\left(\mathrm{C}_{\mathrm{Ar}}\right), 149.0\left(\mathrm{C}_{\mathrm{Ar}}\right), 148.2$ $\left(\mathrm{CH}_{\mathrm{Ar}}\right), 134.5\left(2 \mathrm{C}, \mathrm{CH}_{\mathrm{Phth}}\right), 131.2$ (2C, $\left.\mathrm{C}_{\mathrm{Phth}}\right), 125.1\left(\mathrm{C}_{\mathrm{Ar}}\right), 123.1\left(2 \mathrm{C}, \mathrm{CH}_{\mathrm{Phth}}\right), 116.6\left(\mathrm{CH}_{\mathrm{Ar}}\right)$, $39.7\left(\mathrm{CH}_{2}\right), 36.7(\mathrm{CH}), 32.5\left(\mathrm{CH}_{2}\right), 28.5\left(\mathrm{CH}_{2}\right)$.

IR (neat), $v\left(\mathrm{~cm}^{-1}\right): 1706,1680,1580,1466,1348,1168,1001,941,722,714$.

HRMS (ESI) m/z: [M + H] $]^{+}$Calcd for $\mathrm{C}_{18} \mathrm{H}_{15} \mathrm{ClN}_{3} \mathrm{O}_{3}$ 356.0796; Found 356.0800.

$\mathrm{R}_{\mathrm{f}}=0.27(\mathrm{PE} / \mathrm{EtOAc}=70 \%)$.

m.p. $=216-219^{\circ} \mathrm{C}($ crystallized from PE/EtOAc) 
<smiles>CC(=O)OCC1CCC(=O)Nc2nc(Cl)ccc21</smiles>

Chemical Formula: $\mathrm{C}_{12} \mathrm{H}_{13} \mathrm{ClN}_{2} \mathrm{O}_{3}$ Molecular Weight: 268.70

Following the general procedure E, the reaction was carried out with the solution of 4ga (199 $\mathrm{mg}, 0.42 \mathrm{mmol}, 1$ eq.) in chlorobenzene $(42 \mathrm{~mL})$ with 5 eq. of DTBP $(0.39 \mathrm{~mL})$ for $6 \mathrm{~h}$. Flash column chromatography on silica gel (eluent: $\mathrm{DCM} / \mathrm{MeOH}=2 \%$ ) afforded target product 5ga $(11.3 \mathrm{mg}, 10 \%)$ as a yellow oil.

${ }^{1} \mathrm{H}$ NMR (400 MHz, Chloroform- $d$ ) $\delta 7.80(\mathrm{~s}, 1 \mathrm{H}, \mathrm{NH}), 7.55\left(\mathrm{~d}, J=8.0 \mathrm{~Hz}, 1 \mathrm{H}, \mathrm{CH}_{\mathrm{Ar}}\right), 7.14$ $\left(\mathrm{d}, J=8.0 \mathrm{~Hz}, 1 \mathrm{H}, \mathrm{CH}_{\mathrm{Ar}}\right), 4.44\left(\mathrm{dd}, J=11.4,7.4 \mathrm{~Hz}, 1 \mathrm{H}, \mathrm{CH}_{A l} \mathrm{H}\right), 4.32(\mathrm{dd}, J=11.4,6.0 \mathrm{~Hz}$, $\left.1 \mathrm{H}, \mathrm{CH}_{A 2}\right), 3.37-3.25(\mathrm{~m}, 1 \mathrm{H}, \mathrm{CH}), 2.58-2.38\left(\mathrm{~m}, 3 \mathrm{H}, \mathrm{CH}_{2}\right.$ and $\left.\mathrm{CH}_{B 1} \mathrm{H}\right), 2.04\left(\mathrm{~s}, 3 \mathrm{H}, \mathrm{CH}_{3}\right)$, $2.02-1.88\left(\mathrm{~m}, 1 \mathrm{H}, \mathrm{CH} H_{B 2}\right)$.

${ }^{13} \mathrm{C}$ NMR (101 MHz, Chloroform- $\left.d\right) \delta 173.3(\mathrm{C}=\mathrm{O}), 170.8(\mathrm{C}=\mathrm{O}), 151.1\left(\mathrm{C}_{\mathrm{Ar}}\right), 148.5\left(\mathrm{C}_{\mathrm{Ar}}\right)$, $139.3\left(\mathrm{CH}_{\mathrm{Ar}}\right), 125.8\left(\mathrm{C}_{\mathrm{Ar}}\right), 120.7\left(\mathrm{CH}_{\mathrm{Ar}}\right), 64.5\left(\mathrm{CH}_{2}\right), 38.0(\mathrm{CH}), 32.9\left(\mathrm{CH}_{2}\right), 30.0\left(\mathrm{CH}_{2}\right), 20.9$ $\left(\mathrm{CH}_{3}\right)$.

IR (neat), $v\left(\mathrm{~cm}^{-1}\right): 2945,1738,1683,1568,1441,1367,1236,1118,1043,768$.

HRMS (ESI) m/z: $[\mathrm{M}+\mathrm{H}]^{+}$Calcd for $\mathrm{C}_{12} \mathrm{H}_{14} \mathrm{ClN}_{2} \mathrm{O}_{3}$ 269.0687; Found 269.0689.

$\mathrm{R}_{\mathrm{f}}=0.41(\mathrm{PE} / \mathrm{EtOAc}=60 \%)$. 


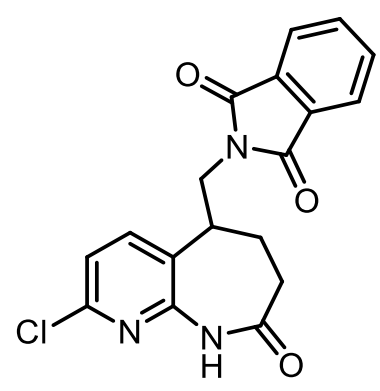

Chemical Formula: $\mathrm{C}_{18} \mathrm{H}_{14} \mathrm{CIN}_{3} \mathrm{O}_{3}$ Molecular Weight: 355.78

Following the general procedure E, the reaction was carried out with the solution of $4 \mathbf{g c}$ (265 $\mathrm{mg}, 0.48 \mathrm{mmol}, 1$ eq.) in chlorobenzene (48 mL) with 5 eq. of DTBP $(0.44 \mathrm{~mL})$ for $3 \mathrm{~h}$. Flash column chromatography on silica gel (eluent: PE/EtOAc $=0 \% \rightarrow 35 \% \rightarrow 55 \%$ ), followed by the crystallization from $\mathrm{Et}_{2} \mathrm{O}$, afforded target product $\mathbf{5 g c}(37 \mathrm{mg}, 22 \%)$ as a white solid.

${ }^{1} \mathrm{H}$ NMR (400 MHz, Chloroform-d) $\delta 7.90$ (s, 1H, NH), 7.82 - 7.78 (m, 2H, CH $\mathrm{CH}_{\text {Phth }}$ ), 7.73 $7.69\left(\mathrm{~m}, 2 \mathrm{H}, \mathrm{CH}_{\mathrm{Phth}}\right), 7.65\left(\mathrm{~d}, \mathrm{~J}=8.0 \mathrm{~Hz}, 1 \mathrm{H}, \mathrm{CH}_{\mathrm{Ar}}\right), 7.05\left(\mathrm{~d}, \mathrm{~J}=8.0 \mathrm{~Hz}, 1 \mathrm{H}, \mathrm{CH}_{\mathrm{Ar}}\right), 4.12$ (dd, $\left.\mathrm{J}=13.7,8.0 \mathrm{~Hz}, 1 \mathrm{H}, \mathrm{CH} H_{A l} \mathrm{H}\right), 3.91\left(\mathrm{dd}, \mathrm{J}=13.7,7.4 \mathrm{~Hz}, 1 \mathrm{H}, \mathrm{CH}_{A 2}\right), 3.60-3.49(\mathrm{~m}, 1 \mathrm{H}$, $\mathrm{CH}), 2.52-2.37\left(\mathrm{~m}, 3 \mathrm{H}, \mathrm{CH}_{2}\right.$ and $\left.\mathrm{CH}_{B 1} \mathrm{H}\right), 2.05-1.93\left(\mathrm{~m}, 1 \mathrm{H}, \mathrm{CH} H_{B 2}\right)$.

${ }^{13} \mathrm{C}$ NMR (101 MHz, Chloroform-d) $\delta 173.2(\mathrm{C}=\mathrm{O}), 168.2(\mathrm{C}=\mathrm{O}), 151.1\left(\mathrm{C}_{\mathrm{Ar}}\right), 148.5\left(\mathrm{C}_{\mathrm{Ar}}\right)$, $139.5\left(\mathrm{CH}_{\mathrm{Ar}}\right), 134.4$ (2C, $\left.\mathrm{CH}_{\text {Phth }}\right), 131.6$ (2C, $\left.\mathrm{C}_{\text {Phth }}\right), 125.7\left(\mathrm{C}_{\text {Ar }}\right), 123.6$ (2C, $\left.\mathrm{CH}_{\text {Phth }}\right), 120.5$ $\left(\mathrm{CH}_{\mathrm{Ar}}\right), 40.3\left(\mathrm{CH}_{2}\right), 37.9(\mathrm{CH}), 32.8\left(\mathrm{CH}_{2}\right), 31.0\left(\mathrm{CH}_{2}\right)$.

IR (neat), $v\left(\mathrm{~cm}^{-1}\right): 1772,1709,1580,1563,1430,1357,1155,968,727$.

HRMS (ESI) m/z: [M + H] $]^{+}$Calcd for $\mathrm{C}_{18} \mathrm{H}_{15} \mathrm{ClN}_{3} \mathrm{O}_{3}$ 356.0796; Found 356.0798.

$\mathrm{R}_{\mathrm{f}}=0.31(\mathrm{PE} / \mathrm{EtOAc}=50 \%)$.

m.p. $=143-144^{\circ} \mathrm{C}\left(\right.$ crystallized from $\left.\mathrm{Et}_{2} \mathrm{O}\right)$. 
(2-methyl-5-(methylsulfonyl)-6-oxo-6,7,8,9-tetrahydro-5H-pyrido[3,2-b]azepin-9yl)methyl acetate (6ca)

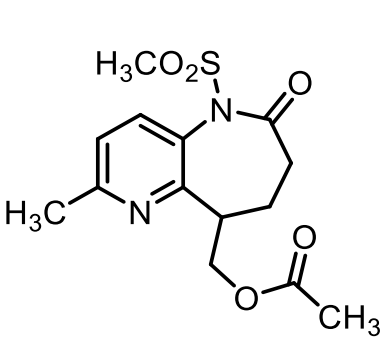

Chemical Formula: $\mathrm{C}_{14} \mathrm{H}_{18} \mathrm{~N}_{2} \mathrm{O}_{5} \mathrm{~S}$

Molecular Weight: 326.37

Following the general procedure E, the reaction was carried out with the solution of 4ca (300 $\mathrm{mg}, 0.67 \mathrm{mmol}, 1$ eq.) in chlorobenzene $(67 \mathrm{~mL})$ with 5 eq. of DTBP $(0.62 \mathrm{~mL})$ for $5 \mathrm{~h}$. Flash column chromatography on silica gel (eluent: $\mathrm{PE} / \mathrm{EtOAc}=50 \%$ ) afforded sulfonylated product 6ca (121 mg, 55\%) as a yellow oil, that solidified thereafter.

${ }^{1} \mathrm{H}$ NMR (400 MHz, Chloroform- $d$ ) $\delta 7.52\left(\mathrm{~d}, J=8.2 \mathrm{~Hz}, 1 \mathrm{H}, \mathrm{CH}_{\mathrm{Ar}}\right), 7.14(\mathrm{~d}, J=8.2 \mathrm{~Hz}, 1 \mathrm{H}$, $\left.\mathrm{CH}_{\mathrm{Ar}}\right), 4.78\left(\mathrm{dd}, J=11.2,5.9 \mathrm{~Hz}, 1 \mathrm{H}, \mathrm{CH}_{A l} \mathrm{H}\right), 4.45\left(\mathrm{dd}, J=11.2,7.8 \mathrm{~Hz}, 1 \mathrm{H}, \mathrm{CH} H_{A 2}\right), 3.53$ $\left(\mathrm{s}, 3 \mathrm{H}, \mathrm{SO}_{2} \mathrm{CH}_{3}\right), 3.50-3.39(\mathrm{~m}, 1 \mathrm{H}, \mathrm{CH}), 2.61-2.52\left(\mathrm{~m}, 1 \mathrm{H}, \mathrm{CH}_{B 1} \mathrm{H}\right), 2.54\left(\mathrm{~s}, 3 \mathrm{H}, \mathrm{CH}_{3}\right)$, $2.51-2.42\left(\mathrm{~m}, 1 \mathrm{H}, \mathrm{CH}_{C l} \mathrm{H}\right), 2.31-2.19\left(\mathrm{~m}, 1 \mathrm{H}, \mathrm{CH} H_{C 2}\right), 2.05\left(\mathrm{~s}, 3 \mathrm{H}, \mathrm{CH}_{3}\right), 1.80-1.69(\mathrm{~m}$, $\left.1 \mathrm{H}, \mathrm{CH} H_{B 2}\right)$.

${ }^{13} \mathrm{C}$ NMR (101 MHz, Chloroform- $\left.d\right) \delta 173.2(\mathrm{C}=\mathrm{O}), 171.3(\mathrm{C}=\mathrm{O}), 159.2\left(\mathrm{C}_{\mathrm{Ar}}\right), 154.1\left(\mathrm{C}_{\mathrm{Ar}}\right)$, $135.7\left(\mathrm{CH}_{\mathrm{Ar}}\right), 129.1\left(\mathrm{C}_{\mathrm{Ar}}\right), 122.0\left(\mathrm{CH}_{\mathrm{Ar}}\right), 65.1\left(\mathrm{CH}_{2}\right), 43.2\left(\mathrm{SO}_{2} \mathrm{CH}_{3}\right), 38.7(\mathrm{CH}), 34.3\left(\mathrm{CH}_{2}\right)$, $30.4\left(\mathrm{CH}_{2}\right), 24.4\left(\mathrm{CH}_{3}\right), 21.1\left(\mathrm{CH}_{3}\right)$.

IR (neat), $v\left(\mathrm{~cm}^{-1}\right): 1731,1706,1460,1350,1236,1163,1128,1033,965,912,763,732$.

HRMS (ESI) m/z: [M + H $]^{+}$Calcd for $\mathrm{C}_{14} \mathrm{H}_{19} \mathrm{~N}_{2} \mathrm{O}_{5} \mathrm{~S}$ 327.1009; Found 327.1009.

$\mathrm{R}_{\mathrm{f}}=0.60(\mathrm{PE} / \mathrm{EtOAc}=70 \%)$. 


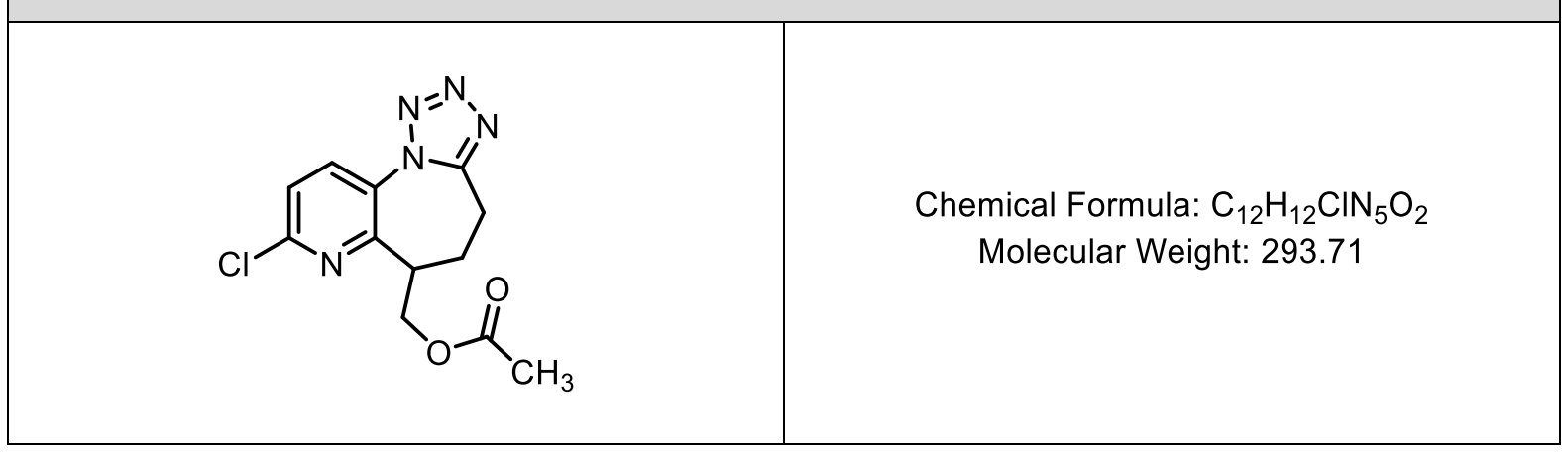

According to a modified literature procedure ${ }^{3}$, to a solution of xanthate 5aa (100 mg, 0.37 mmol, 1 eq. $)$ in pyridine $(0.56 \mathrm{~mL})$ was added diphenylphosphoryl azide $(0.16 \mathrm{~mL}, 0.74 \mathrm{mmol}$, 2 eq.), and the reaction mixture was refluxed under nitrogen for $15 \mathrm{~h}$. Then it was diluted with EtOAc $(30 \mathrm{~mL})$ and washed with sat. aq. $\mathrm{NH}_{4} \mathrm{Cl}$ solution $(3 \times 30 \mathrm{~mL})$. The organic layer was washed with brine, dried over $\mathrm{Na}_{2} \mathrm{SO}_{4}$ and concentrated. The residue was subjected to a column chromatography on silica gel (eluent: $\mathrm{PE} / \mathrm{EtOAc}=25 \% \rightarrow 45 \%$ ) to yield target product 7 (76 $\mathrm{mg}, 69 \%$ ) as a white solid.

${ }^{1} \mathrm{H}$ NMR (400 MHz, Chloroform-d) $\delta 8.12$ (d, J = 8.4 Hz, 1H, $\mathrm{CH}_{\mathrm{Ar}}$ ), 7.49 (d, J = 8.4 Hz, 1H, $\left.\mathrm{CH}_{\mathrm{Ar}}\right), 4.67\left(\mathrm{dd}, \mathrm{J}=11.4,5.9 \mathrm{~Hz}, 1 \mathrm{H}, \mathrm{CH}_{A l} \mathrm{H}\right), 4.37\left(\mathrm{dd}, \mathrm{J}=11.4,7.2 \mathrm{~Hz}, 1 \mathrm{H}, \mathrm{CH} H_{A 2}\right), 3.55-$ $3.45\left(\mathrm{~m}, 1 \mathrm{H}, \mathrm{CH}_{B l} \mathrm{H}\right), 3.19-3.07(\mathrm{~m}, 1 \mathrm{H}, \mathrm{CH}), 2.97-2.84\left(\mathrm{~m}, 1 \mathrm{H}, \mathrm{CH} H_{B 2}\right), 2.69-2.57(\mathrm{~m}$, $\left.1 \mathrm{H}, \mathrm{CH}_{C l} \mathrm{H}\right), 2.29-2.17\left(\mathrm{~m}, 1 \mathrm{H}, \mathrm{CH}_{C 2}\right), 1.99\left(\mathrm{~s}, 3 \mathrm{H}, \mathrm{CH}_{3}\right)$.

${ }^{13} \mathrm{C}$ NMR (101 MHz, Chloroform-d) $\delta 170.8(\mathrm{C}=\mathrm{O}), 154.4\left(\mathrm{C}_{\mathrm{Ar}}\right), 153.0(\mathrm{CAr}), 151.6(\mathrm{C}=\mathrm{N})$, 133.6 $\left(\mathrm{CH}_{\mathrm{Ar}}\right), 129.2\left(\mathrm{C}_{\mathrm{Ar}}\right), 124.2\left(\mathrm{CH}_{\mathrm{Ar}}\right), 64.4\left(\mathrm{CH}_{2}\right), 41.3(\mathrm{CH}), 30.6\left(\mathrm{CH}_{2}\right), 20.9\left(\mathrm{CH}_{3}\right), 20.4$ $\left(\mathrm{CH}_{2}\right)$.

IR (neat), $v\left(\mathrm{~cm}^{-1}\right): 2951,1735,1468,1368,1235,1144,1079,1040,921,732$.

HRMS (ESI) m/z: [M + H] $]^{+}$Calcd for $\mathrm{C}_{12} \mathrm{H}_{13} \mathrm{ClN}_{5} \mathrm{O}_{2}$ 294.0752; Found 294.0752.

$\mathrm{R}_{\mathrm{f}}=0.44(\mathrm{PE} / \mathrm{EtOAc}=50 \%)$.

m.p. $=117-119^{\circ} \mathrm{C}($ crystallized from PE/EtOAc). 
(2,7,7-trichloro-6-oxo-6,7,8,9-tetrahydro-5H-pyrido[3,2-b]azepin-9-yl)methyl acetate

(8)

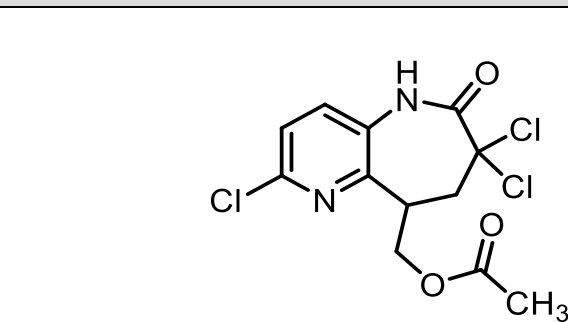

Chemical Formula: $\mathrm{C}_{12} \mathrm{H}_{11} \mathrm{Cl}_{3} \mathrm{~N}_{2} \mathrm{O}_{3}$

Molecular Weight: 337.58

According to a modified literature procedure ${ }^{4}$, to a solution of xanthate 5aa (100 mg, 0.37 mmol, 1 eq.) in toluene (3.7 mL) was added $\mathrm{PCl}_{5}(317 \mathrm{mg}, 1.52 \mathrm{mmol}, 4$ eq.), and the reaction mixture was stirred at $80^{\circ} \mathrm{C}$ under nitrogen for $1.5 \mathrm{~h}$. Then it was cooled to $\mathrm{rt}$ and concentrated under reduced pressure. The residue was diluted with EtOAc $(20 \mathrm{~mL})$ and washed with sat. aq. $\mathrm{NaHCO}_{3}$ solution $(20 \mathrm{~mL})$. Aqueous layer was extracted with EtOAc $(2 \times 20 \mathrm{~mL})$ and the combined organic layers were washed with brine, dried over $\mathrm{Na}_{2} \mathrm{SO}_{4}$ and concentrated. The residue was subjected to a column chromatography on silica gel with (eluent: PE/EtOAc $=15 \%$ $\rightarrow 35 \%)$ to yield target product $\mathbf{8}(80 \mathrm{mg}, 64 \%)$ as a yellow foam. For analytical purposes it was recrystallized from $\mathrm{Et}_{2} \mathrm{O}$ to give white crystals.

1H NMR (400 MHz, Chloroform-d) $\delta 8.11$ (s, 1H, NH), 7.30 (d, J = 8.4 Hz, 1H, CH $\mathrm{CH}_{\mathrm{Ar}}$ ), 7.26 $\left(\mathrm{d}, \mathrm{J}=8.4 \mathrm{~Hz}, 1 \mathrm{H}, \mathrm{CH}_{\mathrm{Ar}}\right), 4.75\left(\mathrm{dd}, \mathrm{J}=11.4,5.1 \mathrm{~Hz}, 1 \mathrm{H}, \mathrm{CH}_{A l} \mathrm{H}\right), 4.42(\mathrm{dd}, \mathrm{J}=11.5,8.2 \mathrm{~Hz}$, $\left.1 \mathrm{H}, \mathrm{CH} H_{A 2}\right), 3.74-3.64(\mathrm{~m}, 1 \mathrm{H}, \mathrm{CH}), 3.44\left(\mathrm{dd}, \mathrm{J}=15.3,6.6 \mathrm{~Hz}, 1 \mathrm{H}, \mathrm{CH}_{B 1} \mathrm{H}\right), 3.08(\mathrm{dd}, \mathrm{J}=$ $\left.15.3,11.9 \mathrm{~Hz}, 1 \mathrm{H}, \mathrm{CH} H_{B 2}\right), 2.08\left(\mathrm{~s}, 3 \mathrm{H}, \mathrm{CH}_{3}\right)$.

${ }^{13} \mathrm{C}$ NMR (101 MHz, Chloroform-d) $\delta 171.0(\mathrm{C}=\mathrm{O}), 165.2(\mathrm{C}=\mathrm{O}), 151.3\left(\mathrm{C}_{\mathrm{Ar}}\right), 147.7\left(\mathrm{C}_{\mathrm{Ar}}\right)$, $132.2\left(\mathrm{C}_{\mathrm{Ar}}\right), 130.9\left(\mathrm{CH}_{\mathrm{Ar}}\right), 123.8\left(\mathrm{CH}_{\mathrm{Ar}}\right), 84.6\left(\mathrm{CCl}_{2}\right), 64.6\left(\mathrm{CH}_{2}\right), 53.2\left(\mathrm{CH}_{2}\right), 39.5(\mathrm{CH}), 21.1$ $\left(\mathrm{CH}_{3}\right)$.

IR (neat), $v\left(\mathrm{~cm}^{-1}\right): 3228,1700,1450,1365,1236,1140,1037,901,835,771$.

HRMS (ESI) m/z: $[\mathrm{M}+\mathrm{H}]^{+}$Calcd for $\mathrm{C}_{12} \mathrm{H}_{12} \mathrm{Cl}_{3} \mathrm{~N}_{2} \mathrm{O}_{3}$ 336.9908; Found 336.9905 .

$\mathrm{R}_{\mathrm{f}}=0.72(\mathrm{PE} / \mathrm{EtOAc}=50 \%)$.

m.p. $=180-181^{\circ} \mathrm{C}\left(\right.$ crystallized from $\left.\mathrm{Et}_{2} \mathrm{O}\right)$. 


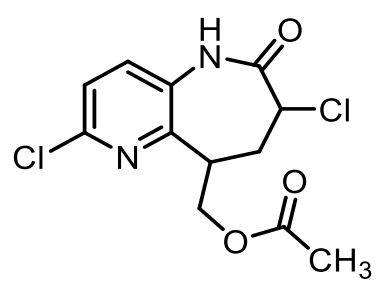

Chemical Formula: $\mathrm{C}_{12} \mathrm{H}_{12} \mathrm{Cl}_{2} \mathrm{~N}_{2} \mathrm{O}_{3}$

Molecular Weight: 303.14

Solution of xanthate $\mathbf{5 a a}(100 \mathrm{mg}, 0.37 \mathrm{mmol}, 1$ eq.) in dry DCM (3.8 mL) was added dropwise to a suspension of $\mathrm{PCl}_{5}(315 \mathrm{mg}, 1.51 \mathrm{mmol}, 4$ eq. $)$ at $0^{\circ} \mathrm{C}$ under argon. The reaction mixture was stirred $2 \mathrm{~h}$, slowly warming to rt. Then it was diluted with DCM (20 mL) and washed with sat. aq. $\mathrm{NaHCO}_{3}$ solution $(20 \mathrm{~mL})$. Aqueous layer was extracted with EtOAc $(3 \times 20 \mathrm{~mL})$ and the combined organic layers were washed with brine, dried over $\mathrm{Na}_{2} \mathrm{SO}_{4}$ and concentrated. The residue was subjected to a column chromatography on silica gel with eluent gradient PE/EtOAc $=10 \% \rightarrow 40 \%$ to yield target product $9(72 \mathrm{mg}, 64 \%)$ as a white solid. For analytical purposes it was crystallized from DCM to give white crystals. Mixture of two diastereomers (d.r. = 1.25:1; determined by the NMR analysis of the crude mixture).

Isomer 1:

${ }^{1} \mathrm{H}$ NMR (400 MHz, Chloroform-d) $\delta 8.85$ (s, 1H, NH), 7.35 (d, J = 7.5 Hz, 1H, CH $\mathrm{CH}_{\mathrm{Ar}}$ ), 7.30 $7.23\left(\mathrm{~m}, 1 \mathrm{H}, \mathrm{CH}_{\mathrm{Ar}}\right), 4.66\left(\mathrm{dd}, \mathrm{J}=11.2,6.1 \mathrm{~Hz}, 1 \mathrm{H}, \mathrm{CH}_{A l} \mathrm{H}\right), 4.37(\mathrm{dd}, \mathrm{J}=11.2,7.8 \mathrm{~Hz}, 1 \mathrm{H}$, $\left.\mathrm{CH}_{A 2}\right), 3.70-3.50(\mathrm{~m}, 1 \mathrm{H}, \mathrm{CH}), 3.19-3.07\left(\mathrm{~m}, 1 \mathrm{H}, \mathrm{CH}_{B 1} \mathrm{H}\right), 2.44-2.31\left(\mathrm{~m}, 1 \mathrm{H}, \mathrm{CH} H_{B 2}\right)$, $2.03\left(\mathrm{~s}, 3 \mathrm{H}, \mathrm{CH}_{3}\right)$.

${ }^{13} \mathrm{C}$ NMR (101 MHz, Chloroform- $\left.d\right) \delta 171.0(\mathrm{C}=\mathrm{O}), 170.3(\mathrm{C}=\mathrm{O}), 153.0\left(\mathrm{C}_{\mathrm{Ar}}\right), 147.6\left(\mathrm{C}_{\mathrm{Ar}}\right)$, $132.3\left(\mathrm{C}_{\mathrm{Ar}}\right), 131.9\left(\mathrm{CH}_{\mathrm{Ar}}\right), 123.7\left(\mathrm{CH}_{\mathrm{Ar}}\right), 64.7\left(\mathrm{CH}_{2}\right), 56.1(\mathrm{CH}), 41.3\left(\mathrm{CH}_{2}\right), 39.9(\mathrm{CH}), 21.0$ $\left(\mathrm{CH}_{3}\right)$.

\section{Isomer 2:}

${ }^{1} \mathrm{H}$ NMR (400 MHz, Chloroform-d) $\delta 9.06$ (s, 1H, NH), 7.40 (d, J = 7.6 Hz, 1H, CHAr), 7.30 $7.27\left(\mathrm{~m}, 1 \mathrm{H}, \mathrm{CH}_{\mathrm{Ar}}\right), 4.73\left(\mathrm{dd}, \mathrm{J}=11.3,5.4 \mathrm{~Hz}, 1 \mathrm{H}, \mathrm{CH}_{A l} \mathrm{H}\right), 4.48-4.41\left(\mathrm{~m}, 2 \mathrm{H}, \mathrm{CH}_{A 2}\right.$ and $\mathrm{CH}), 3.67-3.50(\mathrm{~m}, 1 \mathrm{H}, \mathrm{CH}), 2.84-2.70\left(\mathrm{~m}, 1 \mathrm{H}, \mathrm{CH}_{B 1} \mathrm{H}\right), 2.65-2.53\left(\mathrm{~m}, 1 \mathrm{H}, \mathrm{CH}_{B 2}\right), 2.06$ (s, $\left.3 \mathrm{H}, \mathrm{CH}_{3}\right)$. 
${ }^{13} \mathrm{C}$ NMR (101 MHz, Chloroform-d) $\delta 171.1(\mathrm{C}=\mathrm{O}), 169.7(\mathrm{C}=\mathrm{O}), 153.2\left(\mathrm{C}_{\mathrm{Ar}}\right), 148.4\left(\mathrm{C}_{\mathrm{Ar}}\right)$, 132.6 $\left(\mathrm{CH}_{\mathrm{Ar}}\right), 131.6\left(\mathrm{C}_{\mathrm{Ar}}\right), 123.7\left(\mathrm{CH}_{\mathrm{Ar}}\right), 64.4\left(\mathrm{CH}_{2}\right), 55.6(\mathrm{CH}), 42.9\left(\mathrm{CH}_{2}\right), 39.1(\mathrm{CH}), 21.0$ $\left(\mathrm{CH}_{3}\right)$.

IR (neat), $v\left(\mathrm{~cm}^{-1}\right): 3218,1699,1446,1368,1240,1194,1139,1039,890,835,757$.

HRMS (ESI) m/z: $[\mathrm{M}+\mathrm{H}]^{+}$Calcd for $\mathrm{C}_{12} \mathrm{H}_{13} \mathrm{Cl}_{2} \mathrm{~N}_{2} \mathrm{O}_{3}$ 303.0298; Found 303.0298.

$\mathrm{R}_{\mathrm{f}}=0.44(\mathrm{PE} / \mathrm{EtOAc}=50 \%)$.

m.p. $=206-209^{\circ} \mathrm{C}($ crystallized from DCM $)$. 


\section{Gram-scale preparation of the pyridoazepinone 5aa}

\section{Step 1.}

A stirred solution of the xanthate $\mathbf{3 a}(3.00 \mathrm{~g}, 8.13 \mathrm{mmol}, 1$ eq. $)$ and allyl acetate $(1.80 \mathrm{~mL}, 16.7$ mmol, 2 eq.) in EtOAc ( $8 \mathrm{~mL}, 1.0 \mathrm{M}$ of the xanthate) was refluxed for $15 \mathrm{~min}$ under nitrogen. A portion of DLP (325 mg, $0.81 \mathrm{mmol}, 0.1 \mathrm{eq}$.) was then added to the refluxing solution, followed by one additional portion ( $325 \mathrm{mg}, 0.81 \mathrm{mmol}, 0.1 \mathrm{eq}$.) after $1 \mathrm{~h}$. The reaction mixture was then refluxed for $1 \mathrm{~h}$ until the starting xanthate was completely consumed. The reaction mixture was cooled to room temperature, concentrated under reduced pressure and the residue was purified by flash column chromatography on silica gel (eluent: PE/EtOAc $=40 \%$ ) to yield the target product $4 \mathbf{a a}(3.38 \mathrm{~g}, 89 \%)$.

\section{Step 2.}

A stirred solution of xanthate 4aa $(3.38 \mathrm{~g}, 7.21 \mathrm{mmol}, 1$ eq.) in chlorobenzene (360 mL, 0.02 $\mathrm{M}$ of the xanthate) was refluxed for $15 \mathrm{~min}$ under nitrogen. DTBP (6.6 mL, $36.0 \mathrm{mmol}, 5 \mathrm{eq}$.) was then added to the refluxing solution, and the reaction mixture was refluxed under nitrogen for $3 \mathrm{~h}$. The reaction mixture was then cooled to the room temperature, concentrated under reduced pressure. The crude mixture was crystallized from the small volume of chlorobenzene, to yield the product 5aa $(1.11 \mathrm{~g}, 57 \%)$ as light brown solid. The mother liquor was concentrated under reduced pressure and purified by column chromatography (eluent: $\mathrm{PE} / \mathrm{EtOAc}=40 \% \rightarrow$ $70 \%$ ) to yield additional portion of $\mathbf{5 a a}(0.15 \mathrm{~g}$, 8\%). Total yield of 5aa: $1.26 \mathrm{~g} \mathrm{(65 \% ).} \mathrm{For}$ analytical purposes the product was recrystallized from EtOAc.

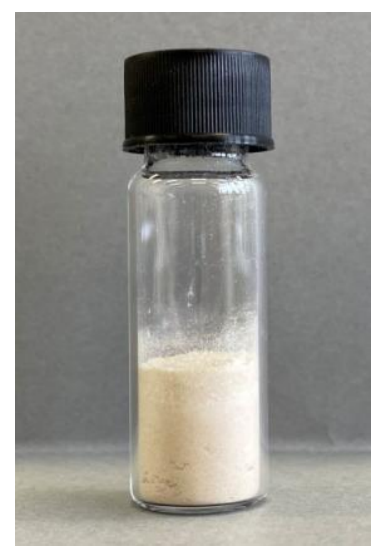




\section{One-pot synthesis of the pyridoazepinone 5aa}

A stirred solution of the xanthate $\mathbf{3 a}(250 \mathrm{mg}, 0.68 \mathrm{mmol}, 1$ eq. $)$ and allyl acetate $(0.15 \mathrm{~mL}$, $1.36 \mathrm{mmol}, 2$ eq.) in chlorobenzene $\left(0.7 \mathrm{~mL}, 1.0 \mathrm{M}\right.$ of the xanthate, degassed with $\left.\mathrm{N}_{2}\right)$ was heated to $90^{\circ} \mathrm{C}$ under nitrogen. A portion of DLP (27 mg, $0.07 \mathrm{mmol}, 0.1 \mathrm{eq}$.) was then added to the refluxing solution, followed by one additional portion $(27 \mathrm{mg}, 0.07 \mathrm{mmol}, 0.1 \mathrm{eq}$.$) after$ $1 \mathrm{~h}$. The reaction mixture was then refluxed for $1 \mathrm{~h}$ until the starting xanthate was completely consumed. The reaction mixture was concentrated by blowing $\mathrm{N}_{2}$ at $90^{\circ} \mathrm{C}$. The residue was diluted with chlorobenzene $\left(34 \mathrm{~mL}, 0.01 \mathrm{M}\right.$, degassed with $\left.\mathrm{N}_{2}\right)$ and DTBP $(0.63 \mathrm{~mL}, 3.43$ mmol, 5 eq.) was added. The reaction mixture was refluxed under nitrogen for $2 \mathrm{~h}$, then cooled down to the room temperature and concentrated under reduced pressure. The residue was subjected to flash column chromatography on silica gel (eluent: PE/EtOAc $=0 \% \rightarrow 50 \% \rightarrow$ $75 \%$ ), then the resulting white solid was washed with pentane (ca. $5 \mathrm{~mL}$ ) to yield the target product 5aa (114 mg, 63\%). 


\section{References}

(1) Kosinski, S.; Wojciechowski, K. Reactions of Pyridine Analogues of Aza-OrthoXylylenes Generated from 1,3-Dialkylpyridosultams. Eur. J. Org. Chem. 2000, 1263-1270.

(2) Huang, Q.; Zard, S. Z. Radical Fragment Coupling Route to Geminal Bis(Boronates). Org. Lett. 2018, 20 (17), 5304-5308.

(3) Ishihara, K.; Shioiri, T.; Matsugi, M. An Expeditious Approach to Tetrazoles from Amides Utilizing Phosphorazidates. Org. Lett. 2020, 22 (16), 6244-6247.

(4) Watthey, J. W. H.; Stanton, J. L.; Desai, M.; Babiarz, J. E.; Finn, B. M. Synthesis and Biological Properties of (Carboxyalkyl)Amino-Substituted Bicyclic Lactam Inhibitors of Angiotensin Converting Enzyme. J. Med. Chem. 1985, 28 (10), 1511-1516. 
Copies of NMR spectra

1H NMR (400 MHz, DMSO)
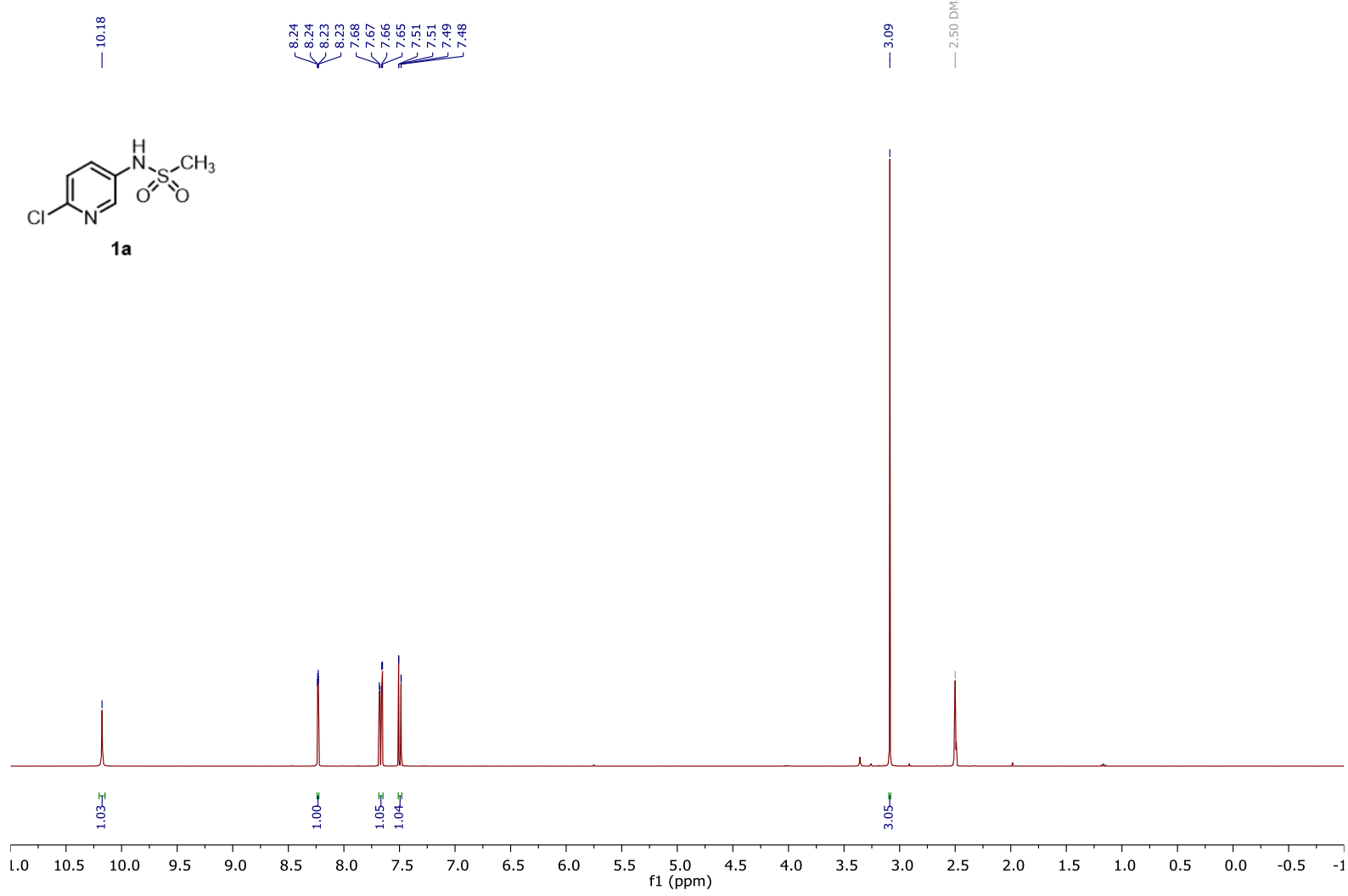

13C NMR (101 MHz, DMSO)

竎亲

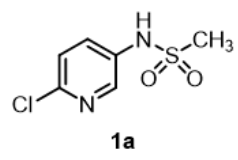

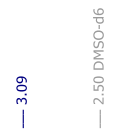

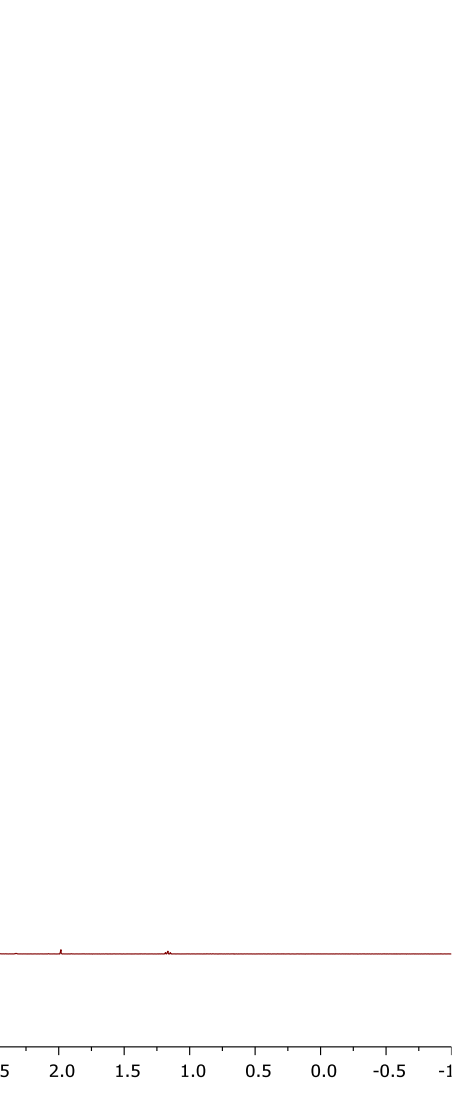

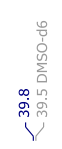
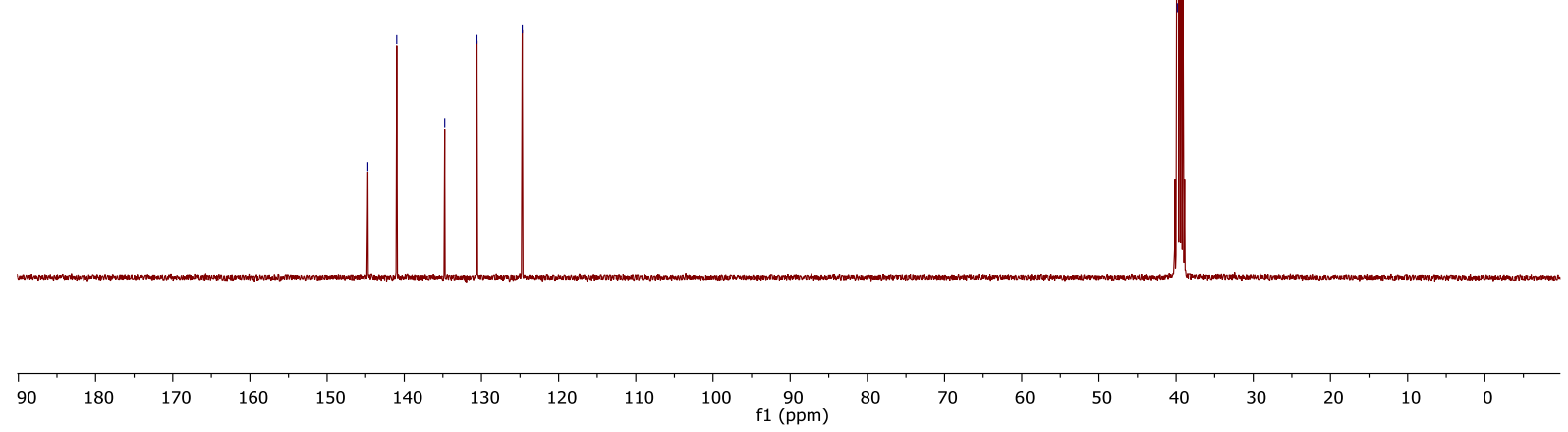

S84 
1H NMR (400 MHz, DMSO)

苛

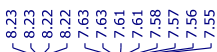<smiles>CS(=O)(=O)Nc1ccc(Br)nc1</smiles>

1b

13C NMR (101 MHz, DMSO)

呈<smiles>CS(=O)(=O)Nc1ccc(Br)nc1</smiles>

1b

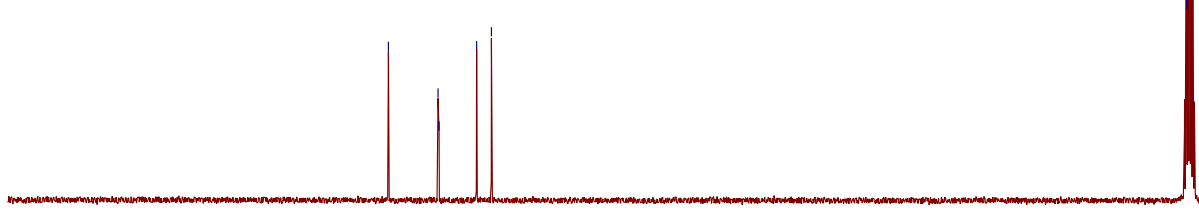

\begin{tabular}{lllllllllllllllllllll}
\hline 90 & 180 & 170 & 160 & 150 & 140 & 130 & 120 & 110 & 100 & 90 & 80 & 70 & 60 & 50 & 40 & 30 & 20 & 10 & 0 & -1
\end{tabular} 
1H NMR (400 MHz, DMSO)

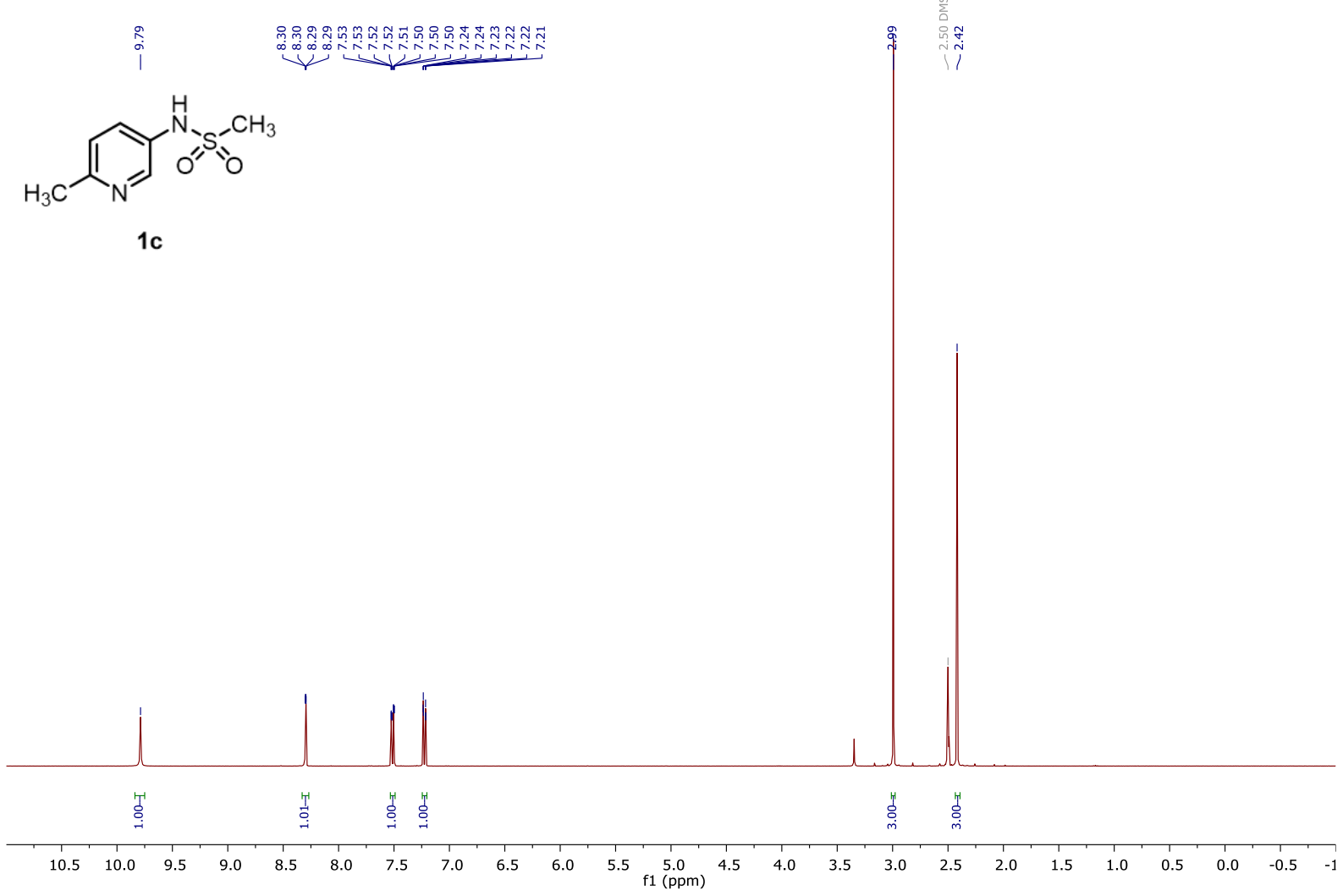

13C NMR (101 MHz, DMSO)<smiles>Cc1ccc(NS(C)(=O)=O)cn1</smiles>

1c

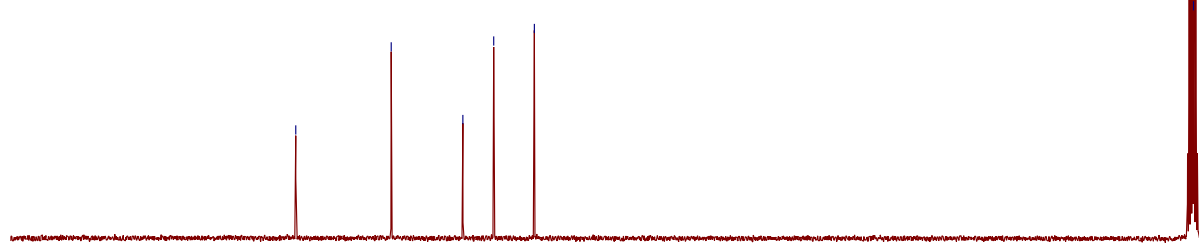

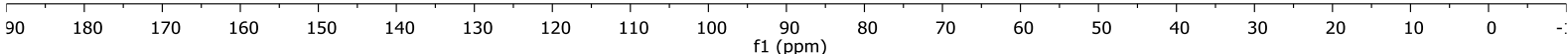


1H NMR (400 MHz, DMSO)

$\stackrel{\circ}{\circ}$

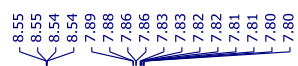

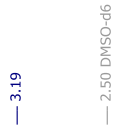

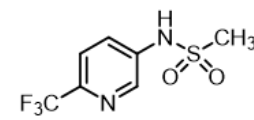

1d

13C NMR (101 MHz, DMSO)

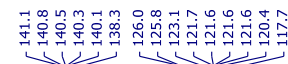

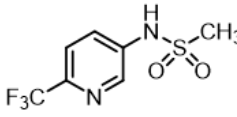

1d

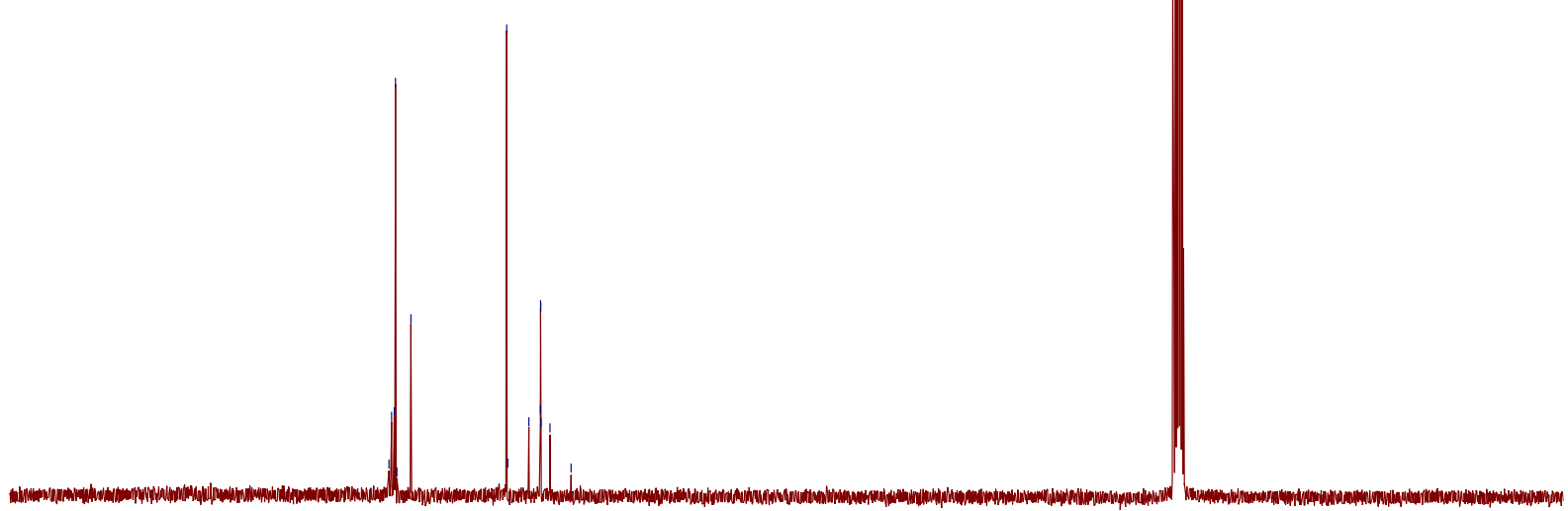

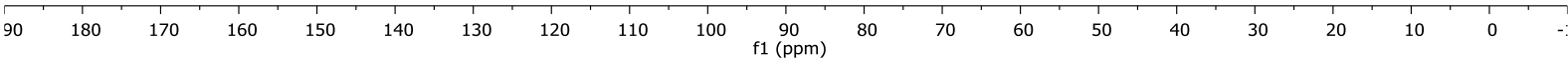


1H NMR (400 MHz, Acetone)
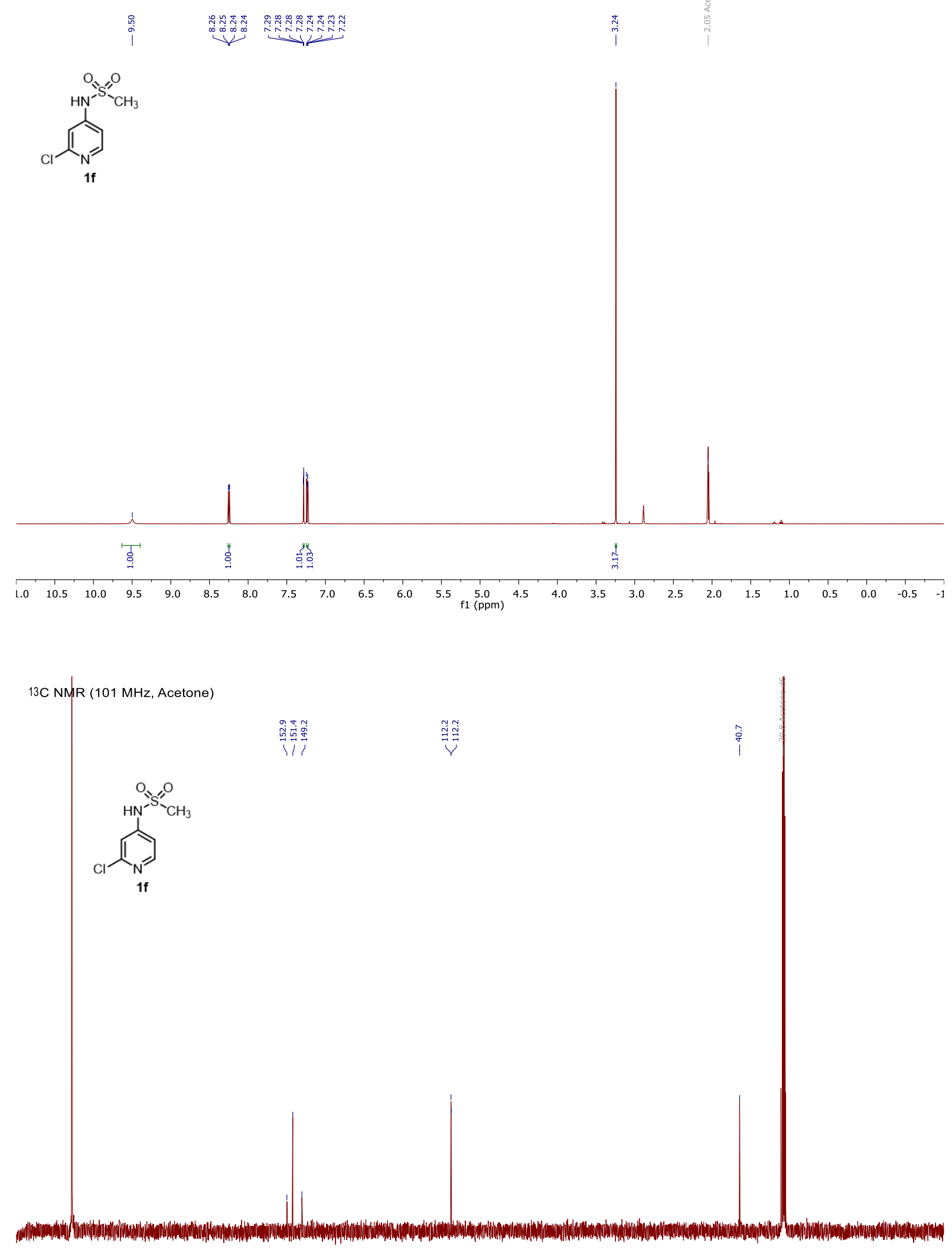

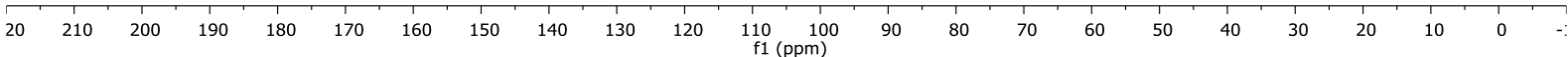




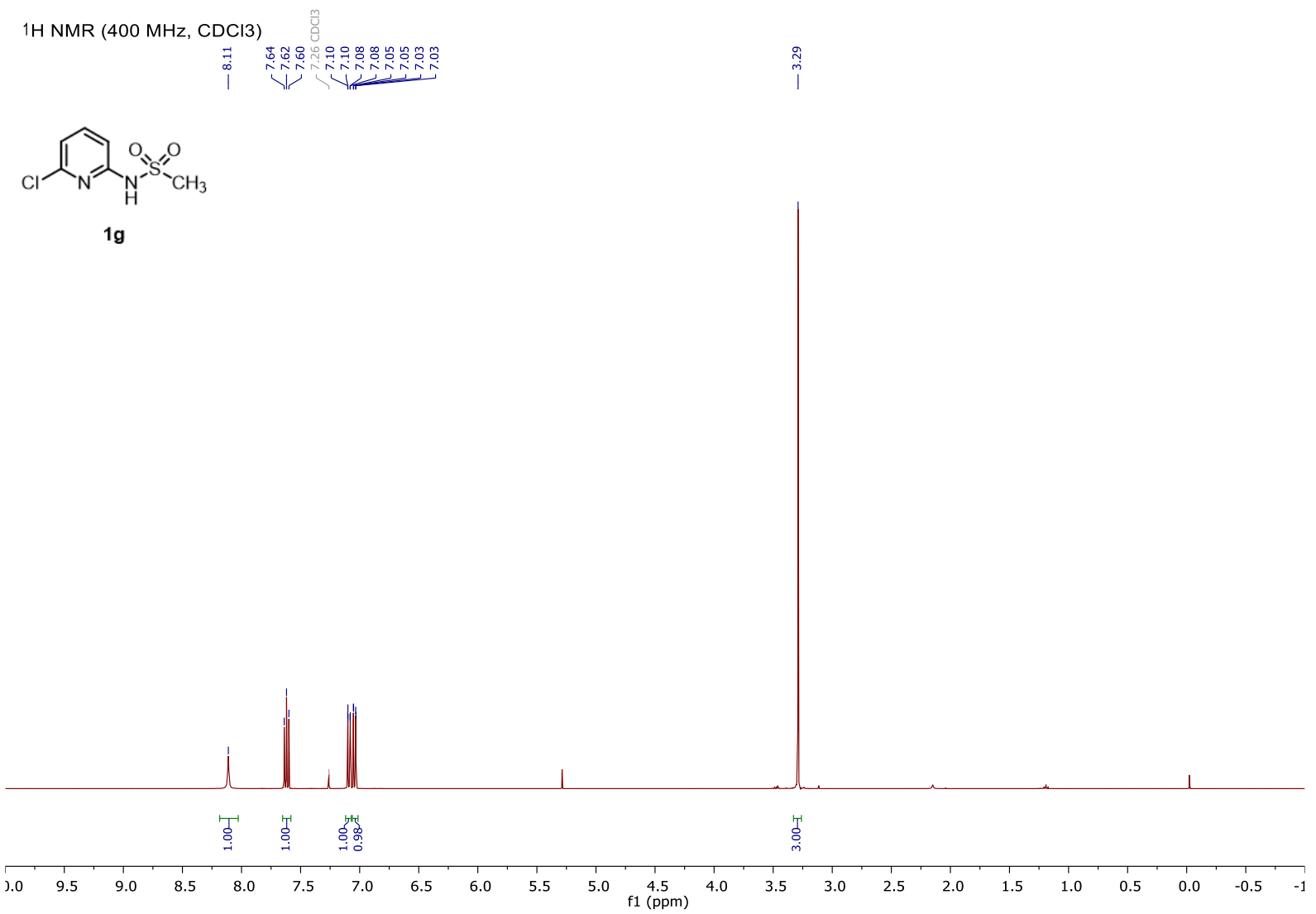

$13 \mathrm{CNMR}(101 \mathrm{MHz}, \mathrm{CDCl3}), 0$

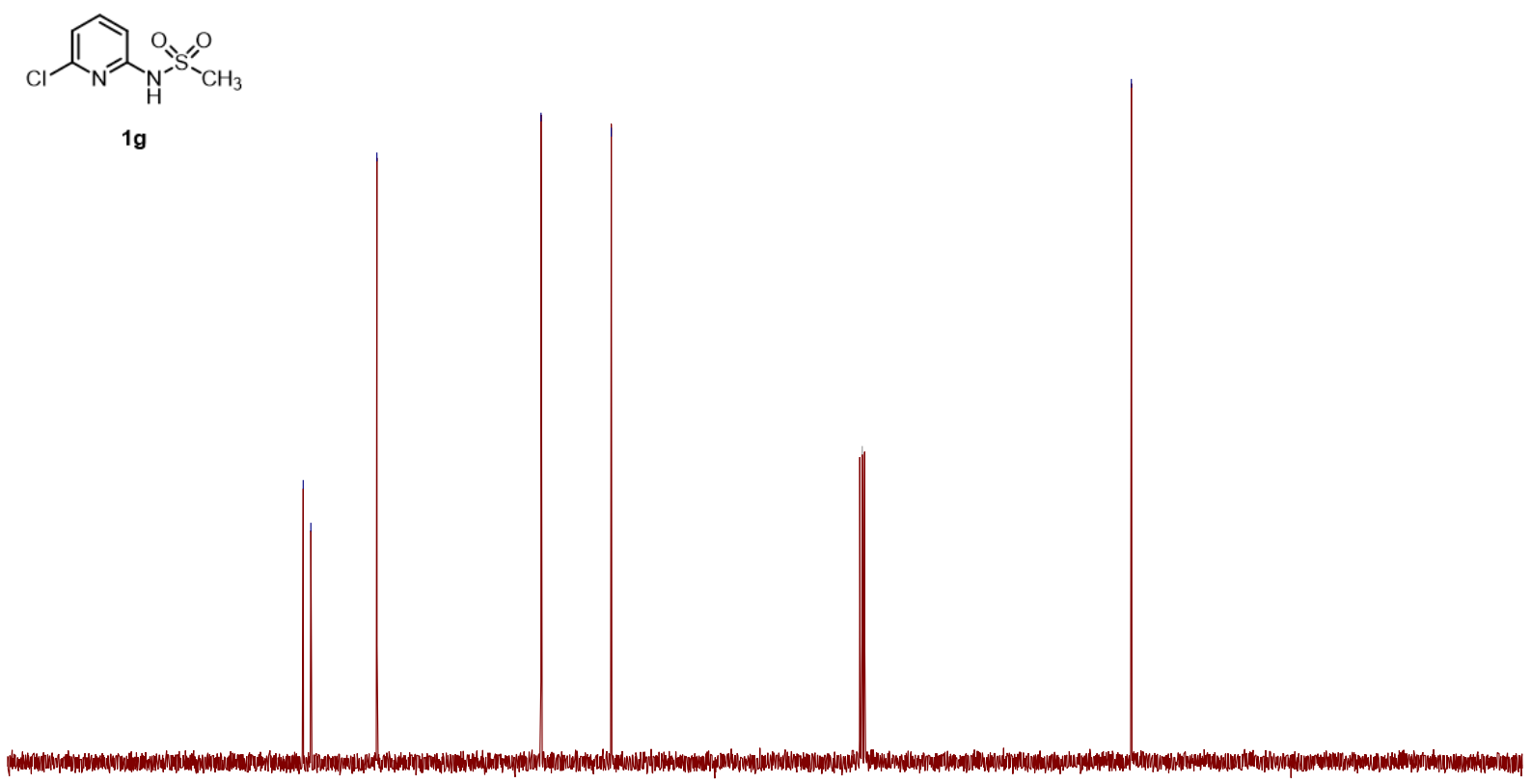

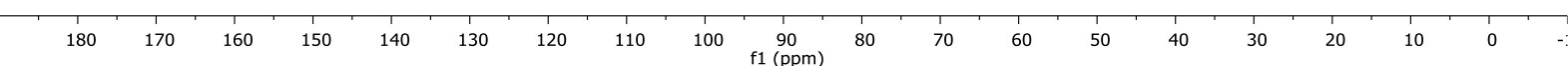


1H NMR (400 MHz, DMSO)

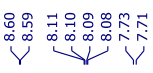

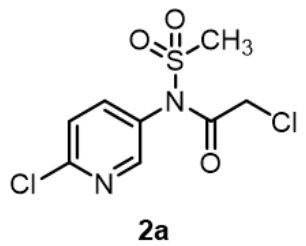

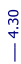

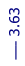

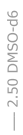

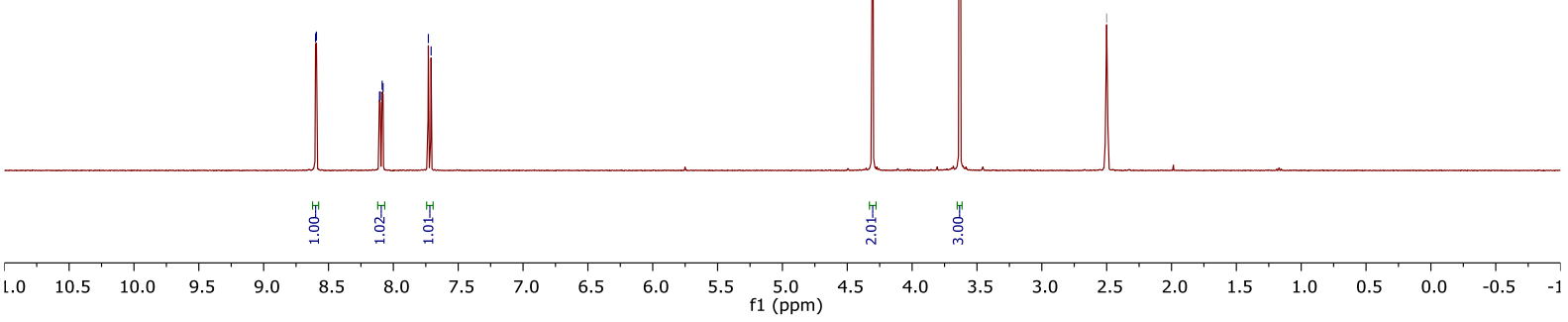

13C NMR (101 MHz, DMSO)
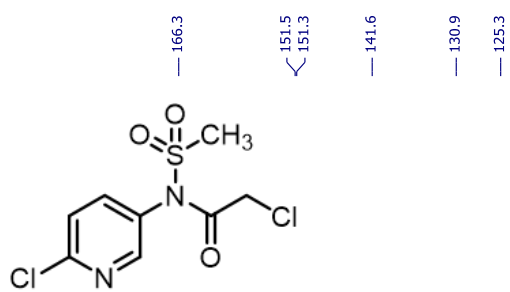

$2 a$
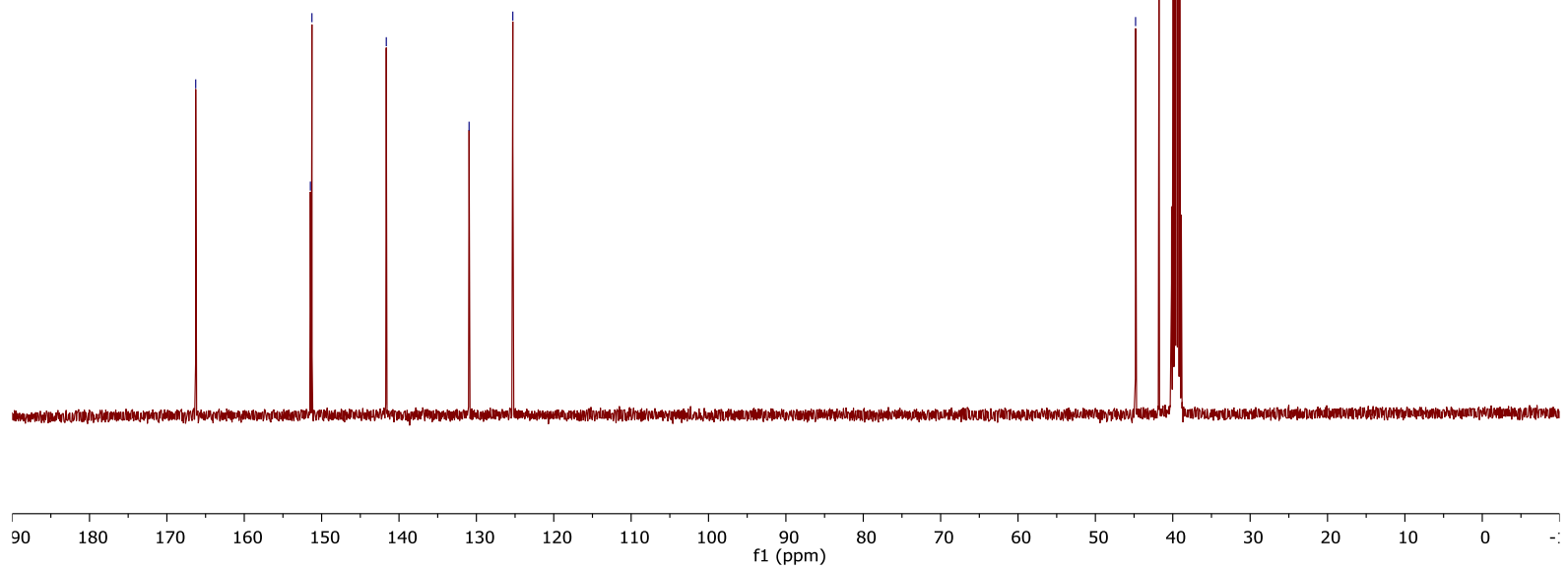

S90 
1H NMR (400 MHz, DMSO)
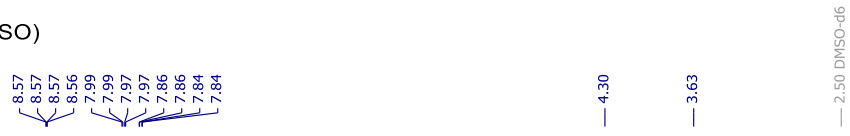<smiles>CS(=O)(=O)N(C(=O)CCl)c1ccc(Br)nc1</smiles>

$2 \mathbf{b}$

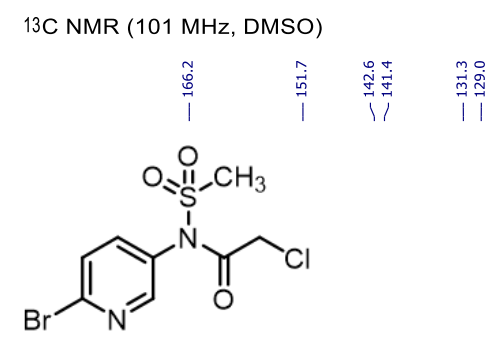

$2 b$

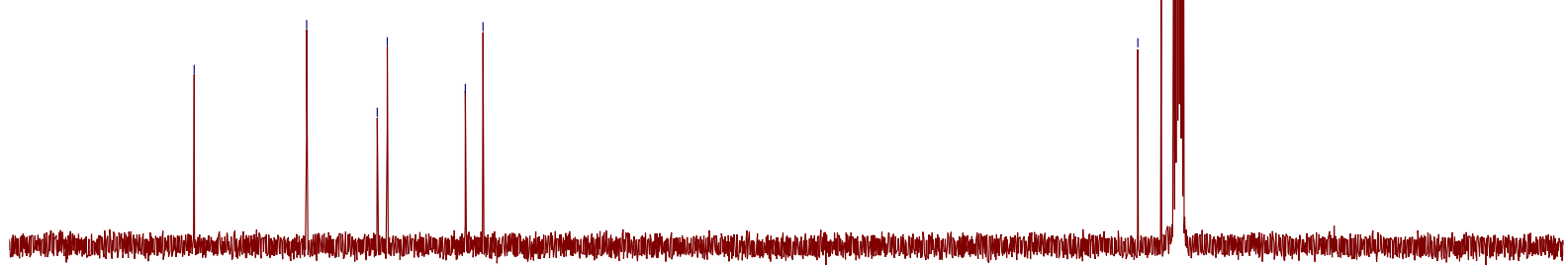

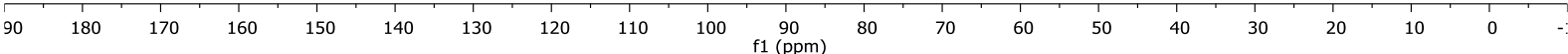




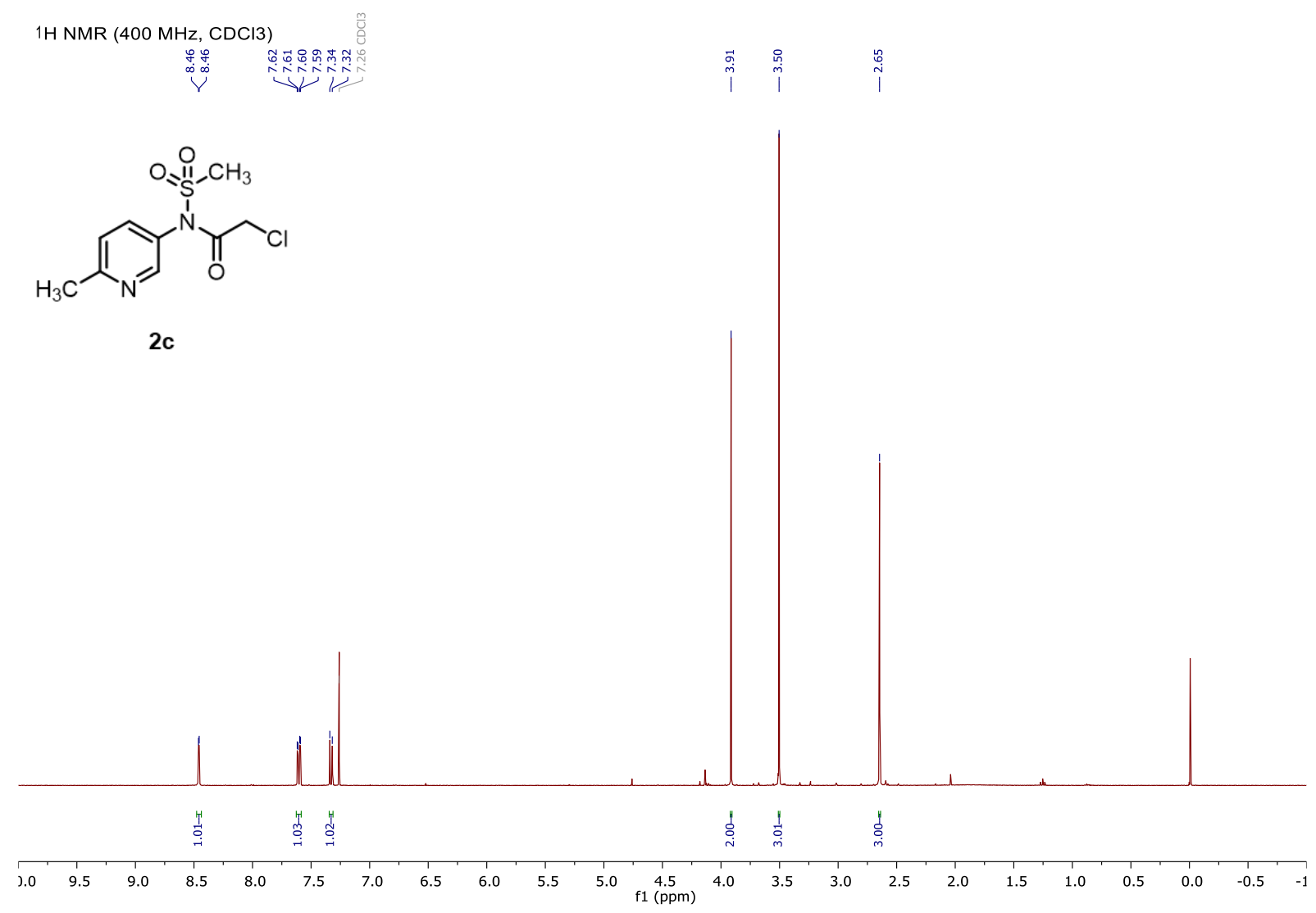

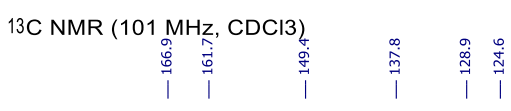<smiles>Cc1ccc(N(C(=O)CCl)S(C)(=O)=O)cn1</smiles>

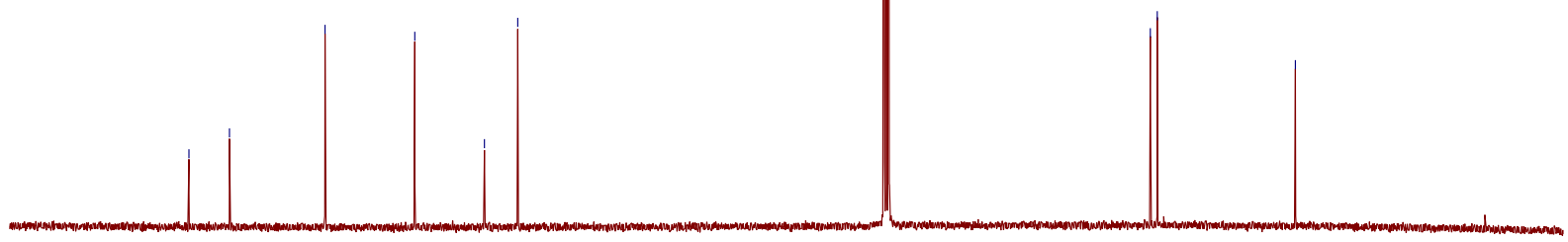

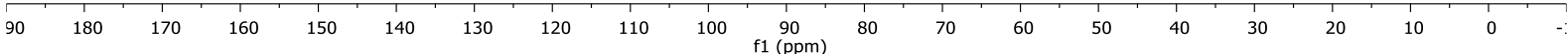


1H NMR (400 MHz, DMSO)

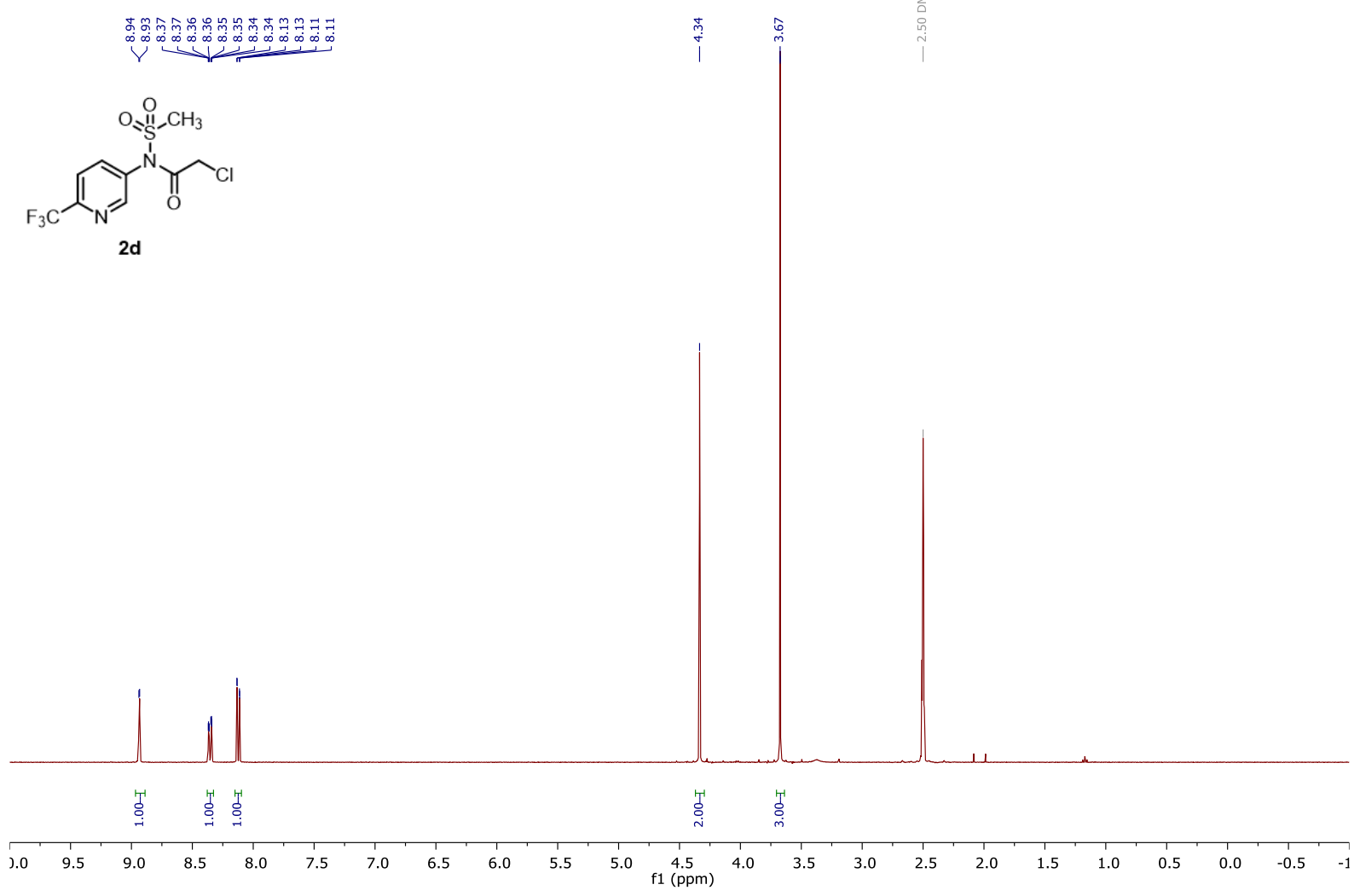

13C NMR (101 MHz, DMSO)

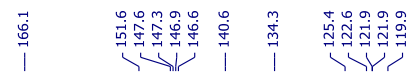<smiles>CC(C)(C)N(C(=O)CCl)c1ccc(C(F)(F)F)nc1</smiles>

2d

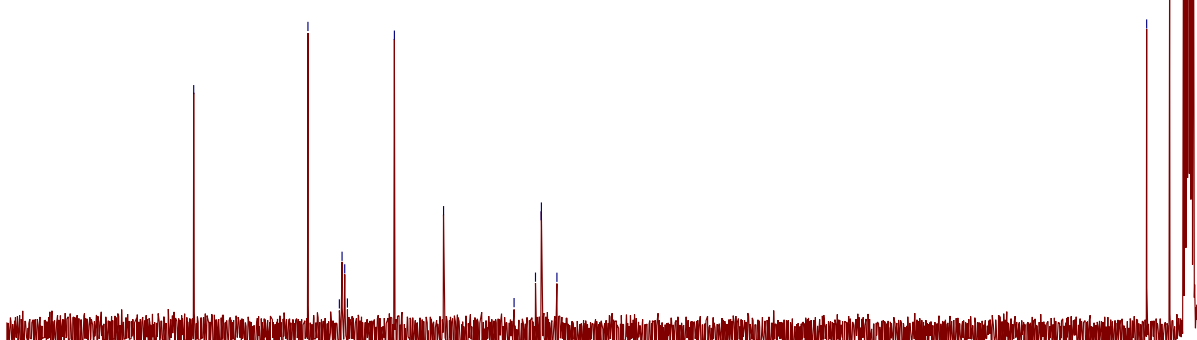

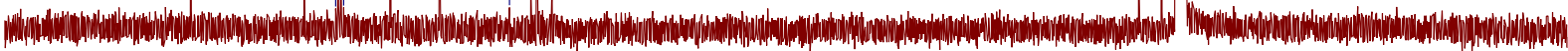

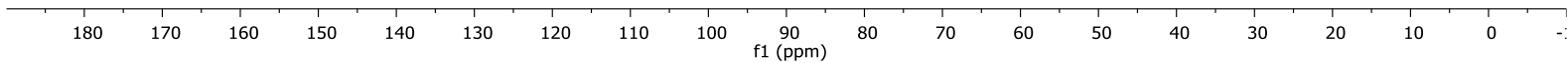


1H NMR (400 MHz, DMSO)

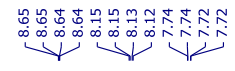

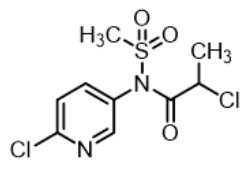

$2 e$

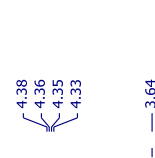

$\sqrt[3]{2 \sqrt[3]{7}}$

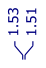

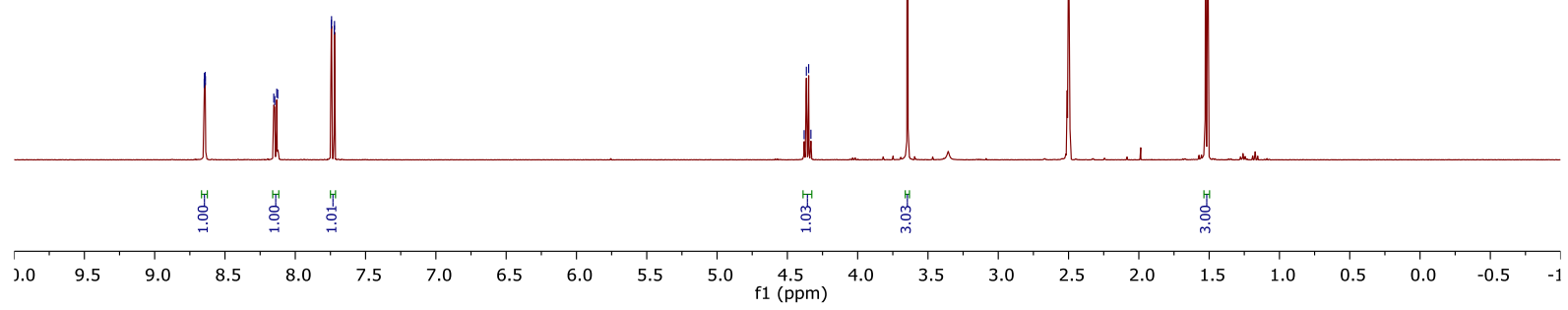

13C NMR (101 MHz, DMSO)
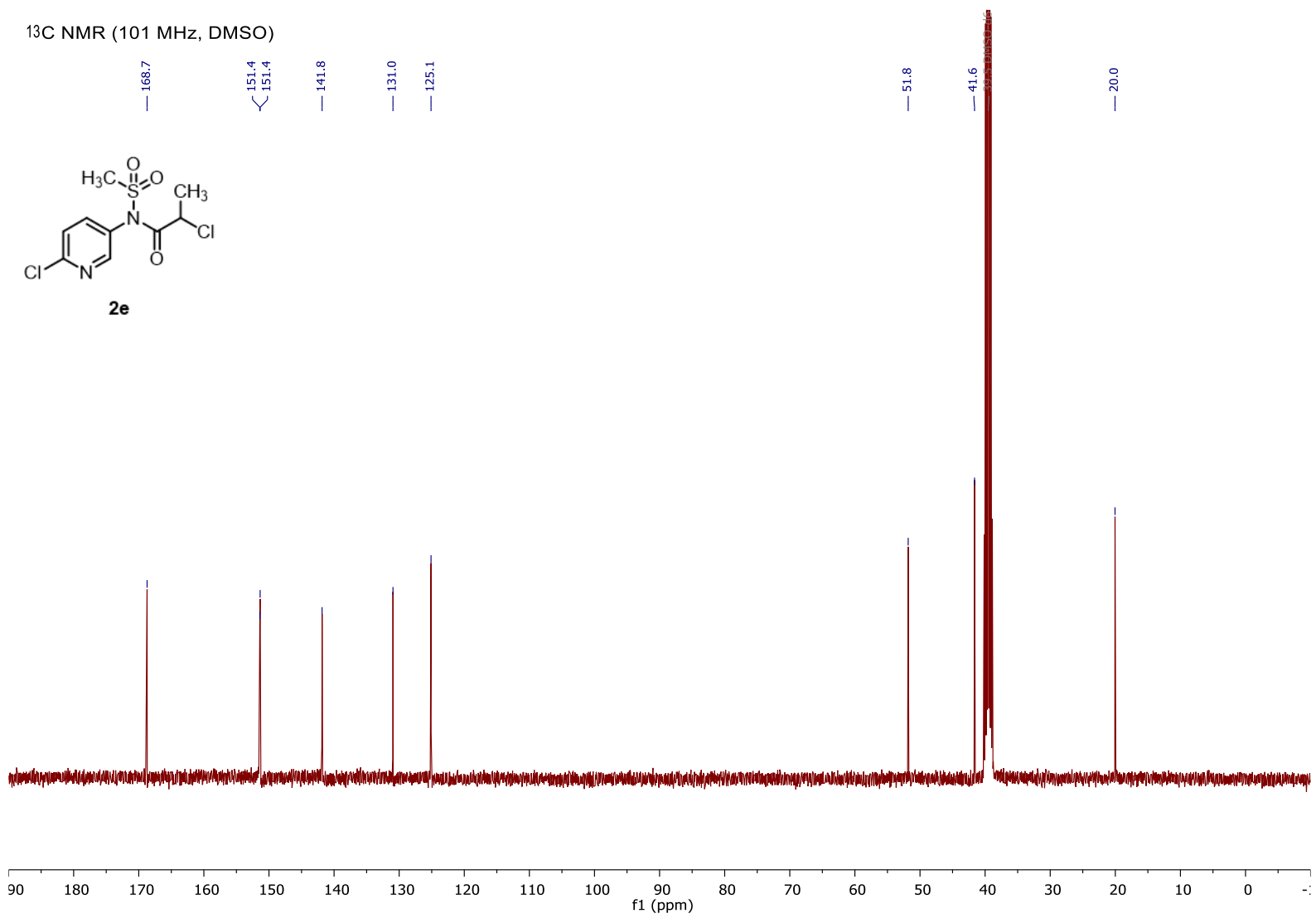

S94 
$1 \mathrm{H}$ NMR $(400 \mathrm{MHz}$, Acetone $)$

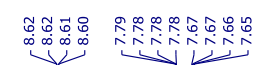<smiles></smiles>
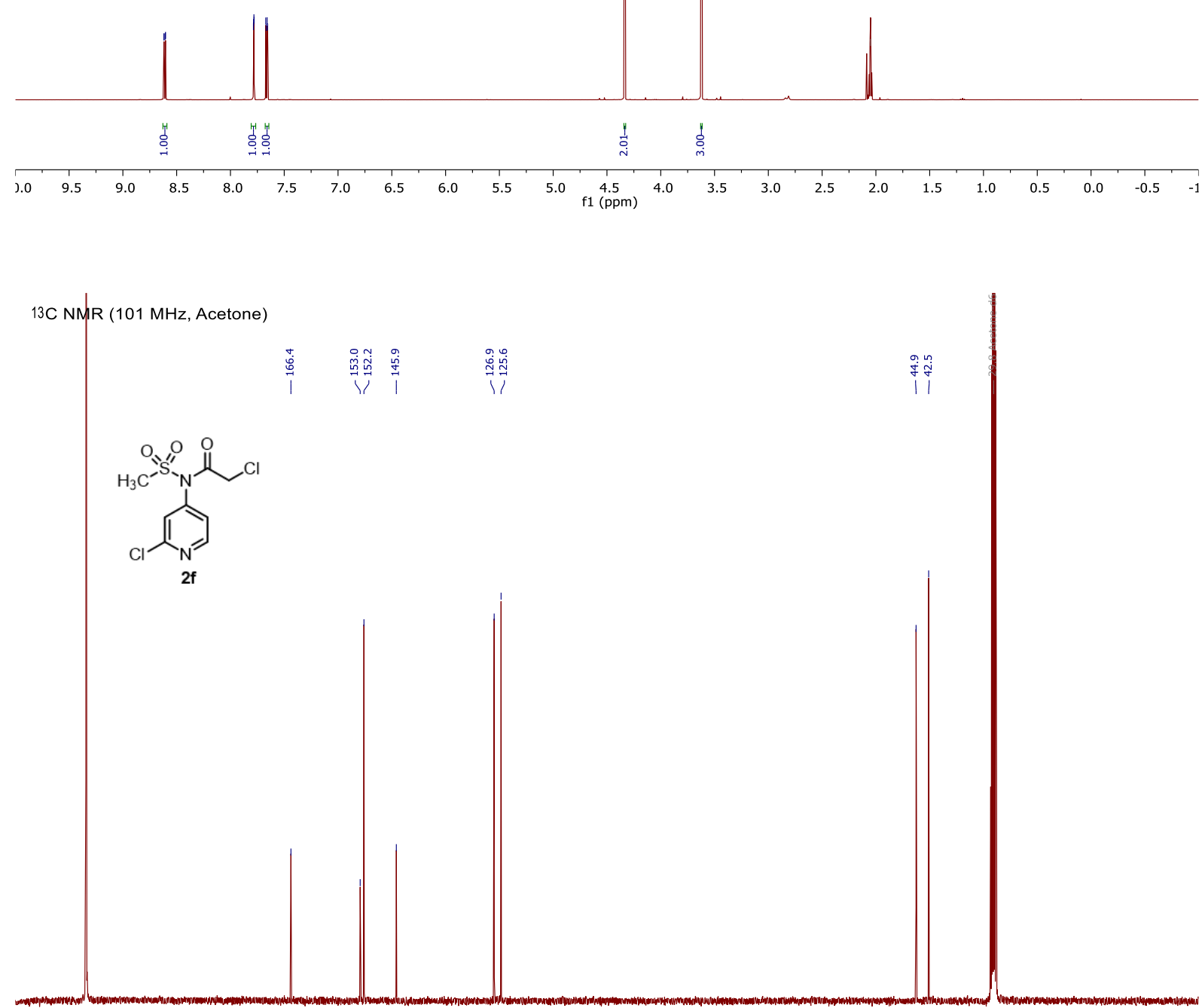

\begin{tabular}{lllllllllllllllllllllllllllllllllll}
\hline 20 & 210 & 200 & 190 & 180 & 170 & 160 & 150 & 140 & 130 & 120 & 110 & 100 & 90 & 80 & 70 & 60 & 50 & 40 & 30 & 20 & 10 & 0 & -
\end{tabular} 
1H NMR (400 MHz, Acetone)

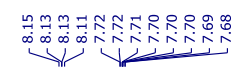

$\stackrel{\vec{m}}{i} \stackrel{\substack{\hat{m} \\ \text { i }}}{1}$

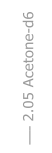

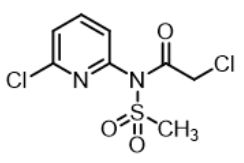

2g
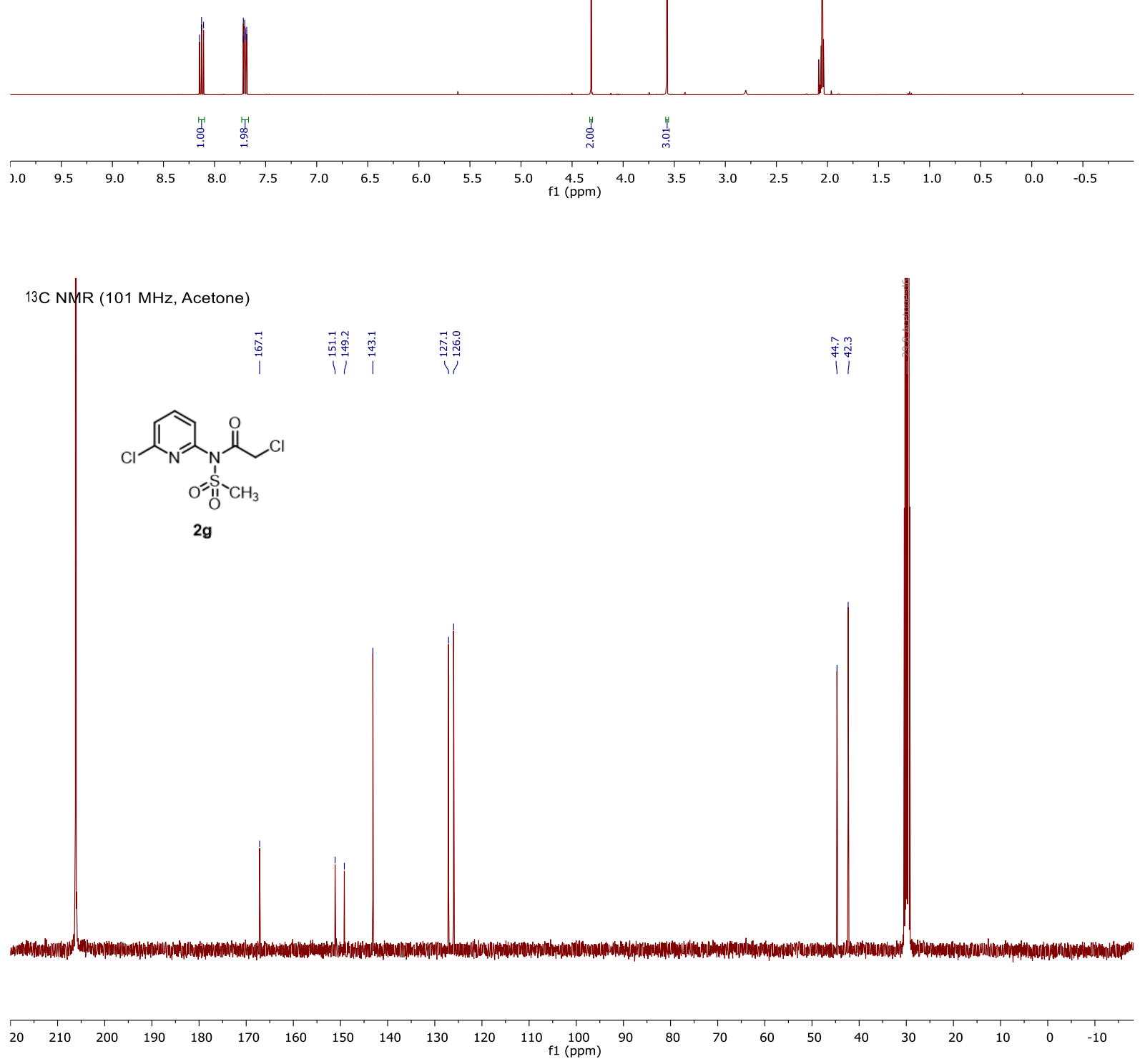

S96 
1H NMR (400 MHz, CDCI3)

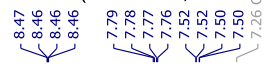

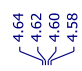

$\stackrel{\substack{\infty \\ j}}{\substack{g \\ m}}$

نَj
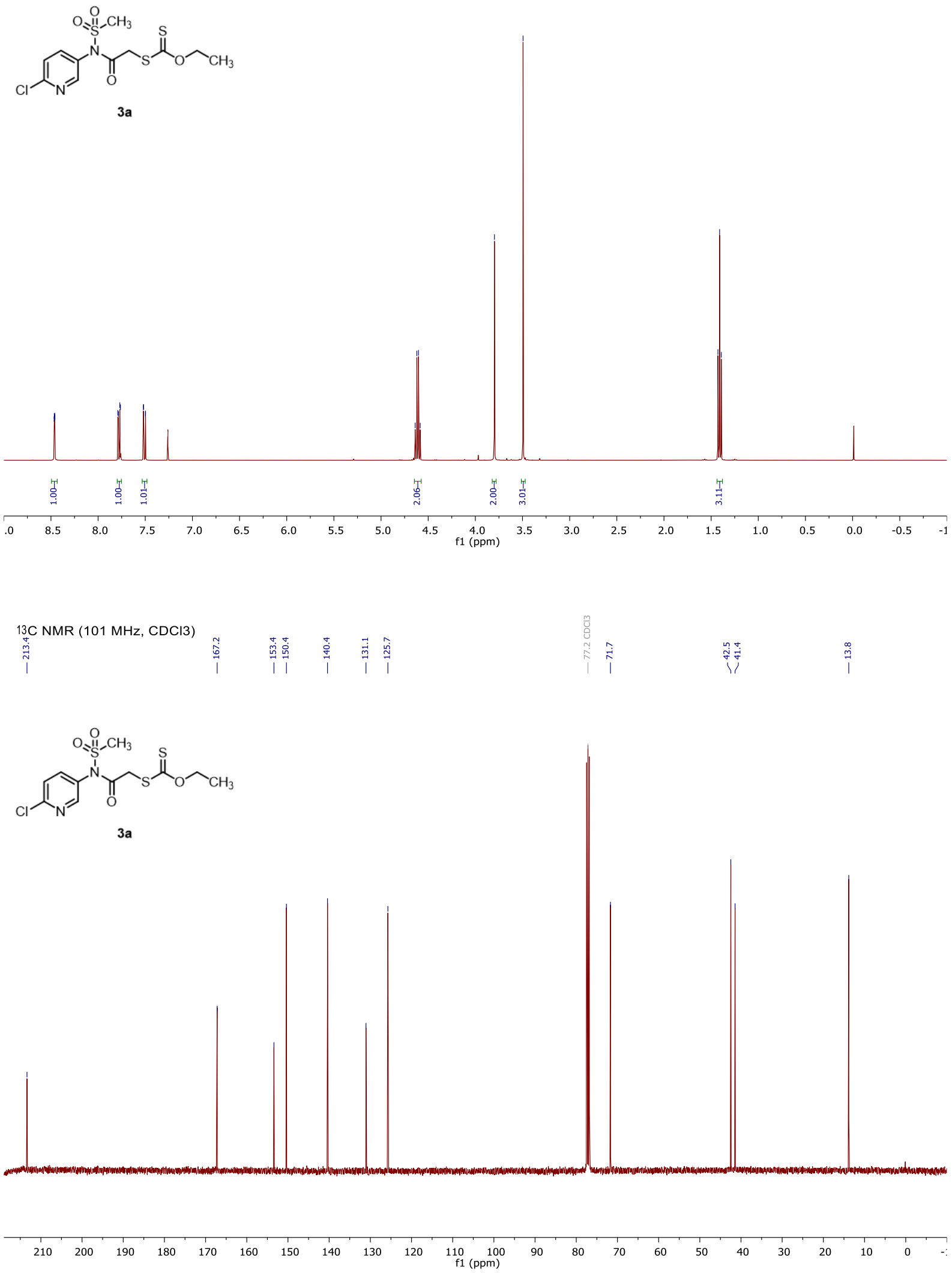

S97 

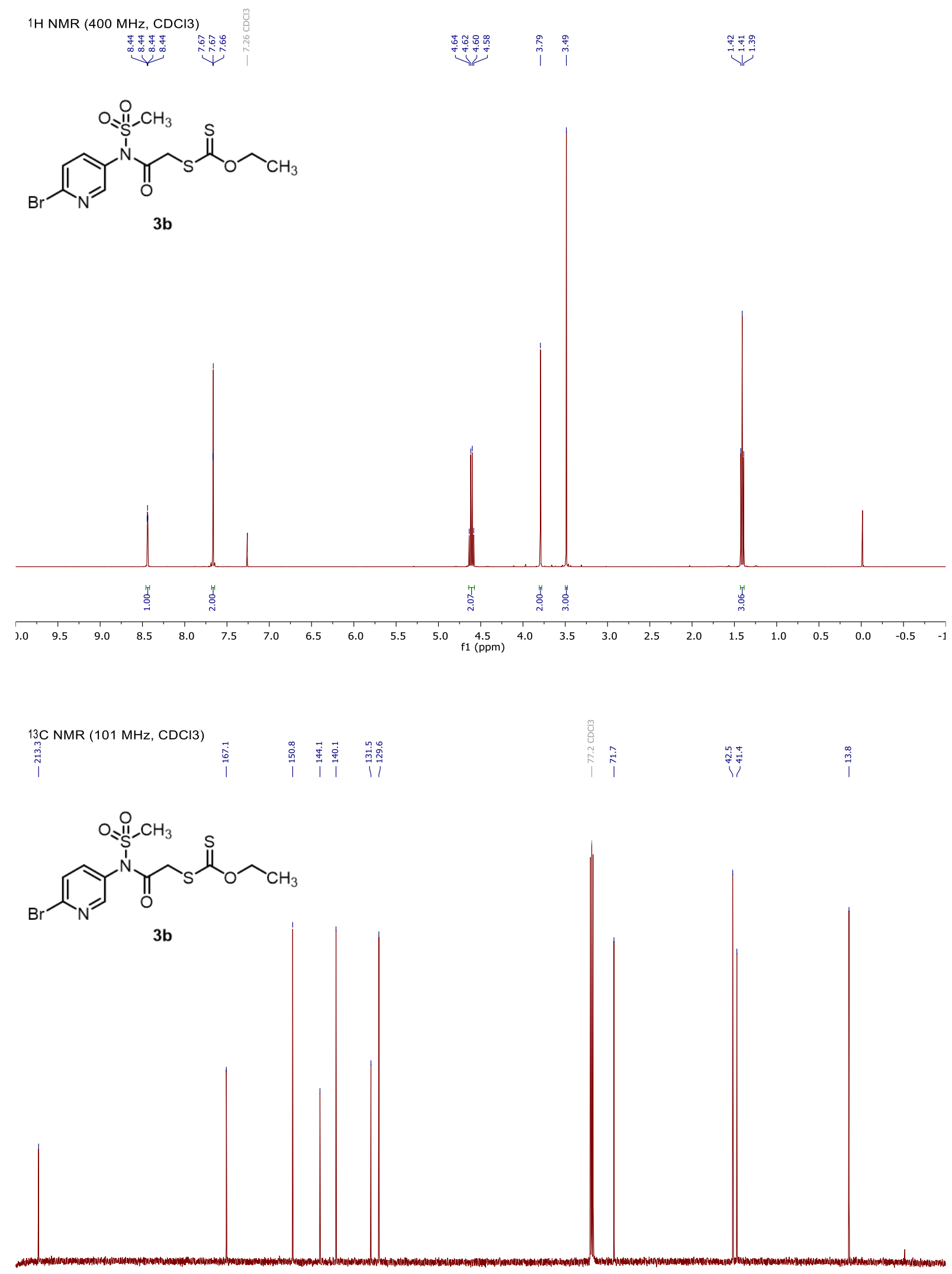

\begin{tabular}{|lllllllllllllllllllllll}
210 & 200 & 190 & 180 & 170 & 160 & 150 & 140 & 130 & 120 & $\underset{\mathrm{f} 1}{110} \underset{(\mathrm{ppm})}{100}$ & 90 & 80 & 70 & 60 & 50 & 40 & 30 & 20 & 10 & 0 & -
\end{tabular} 


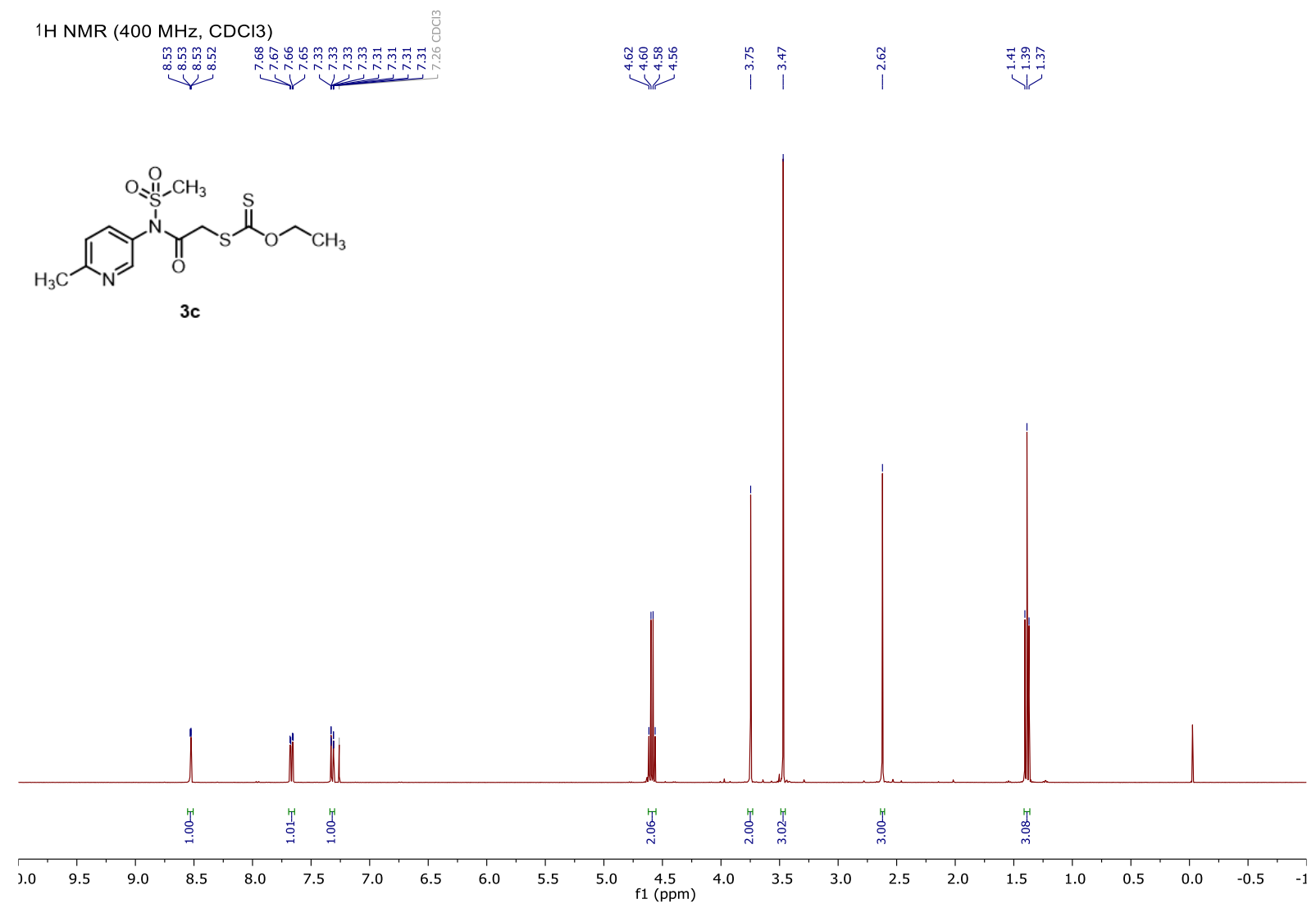

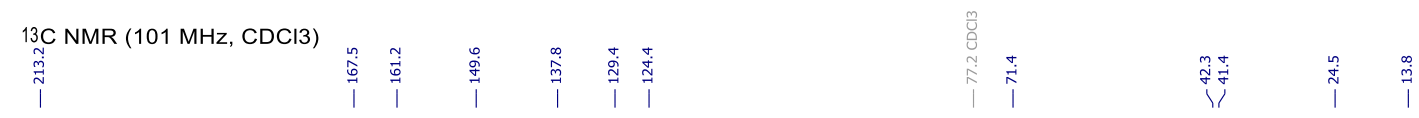<smiles>CCOC(=S)SCC(=O)N(c1ccc(C)nc1)S(C)(=O)=O</smiles>

$3 c$

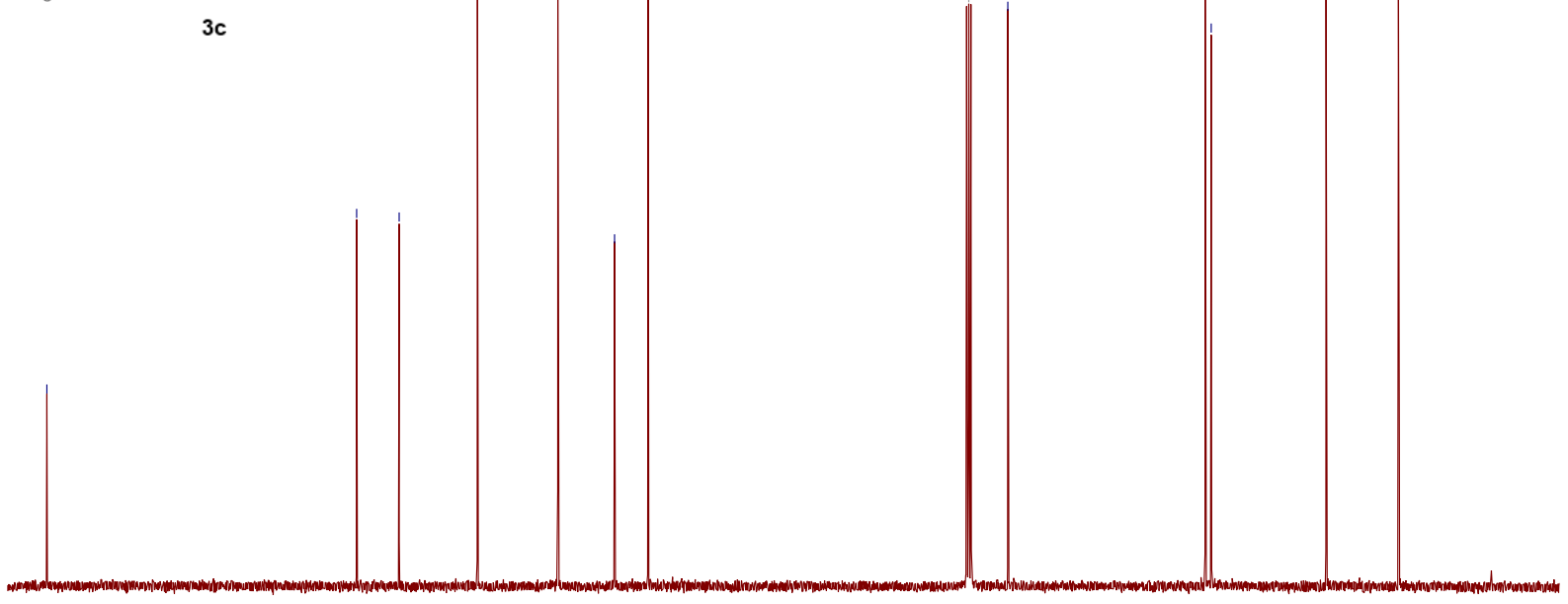

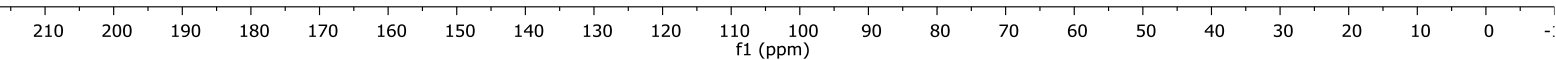



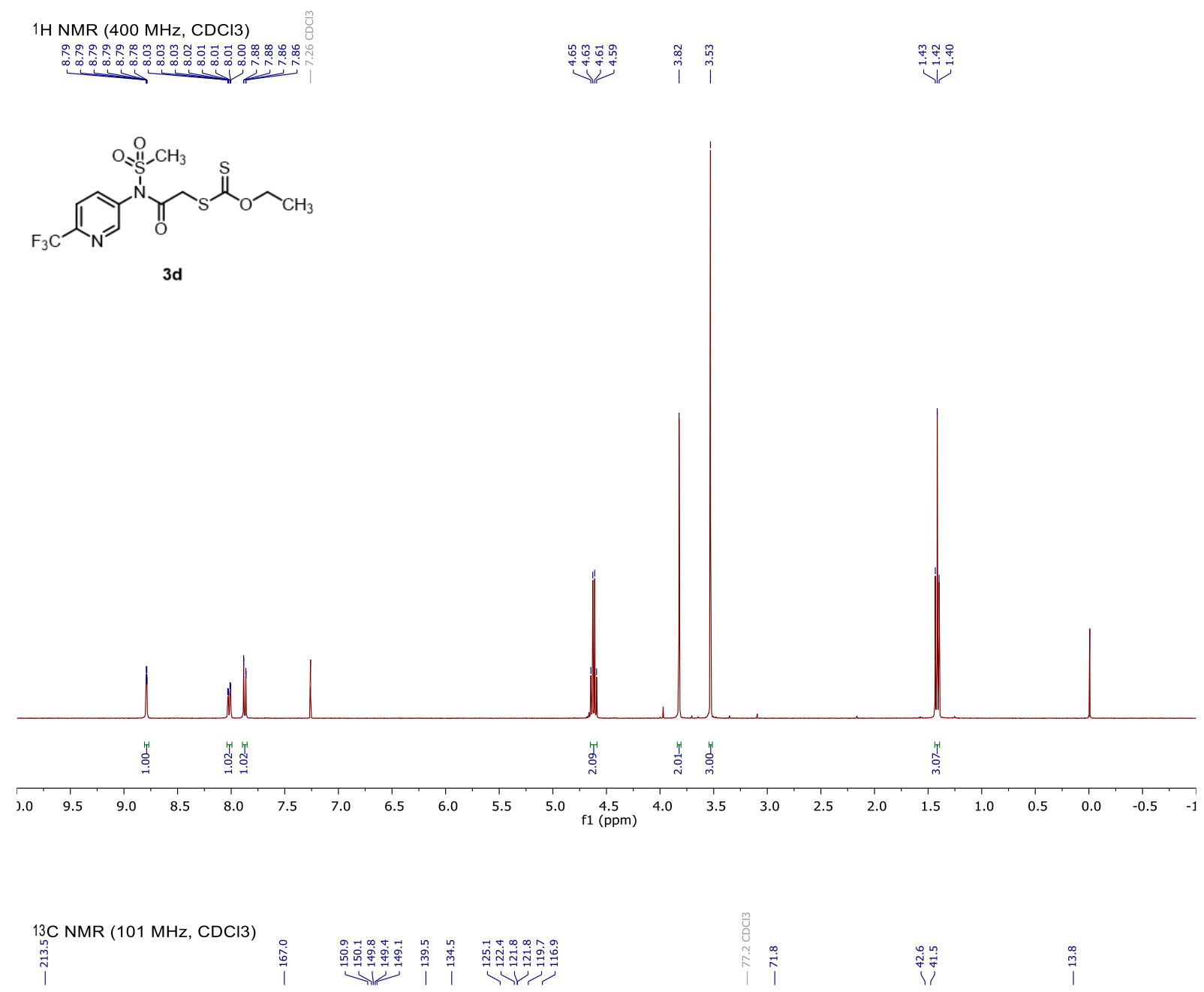<smiles>CCOC(=S)SCC(=O)N(c1ccc(C(F)(F)F)nc1)S(C)(=O)=O</smiles>

$3 d$

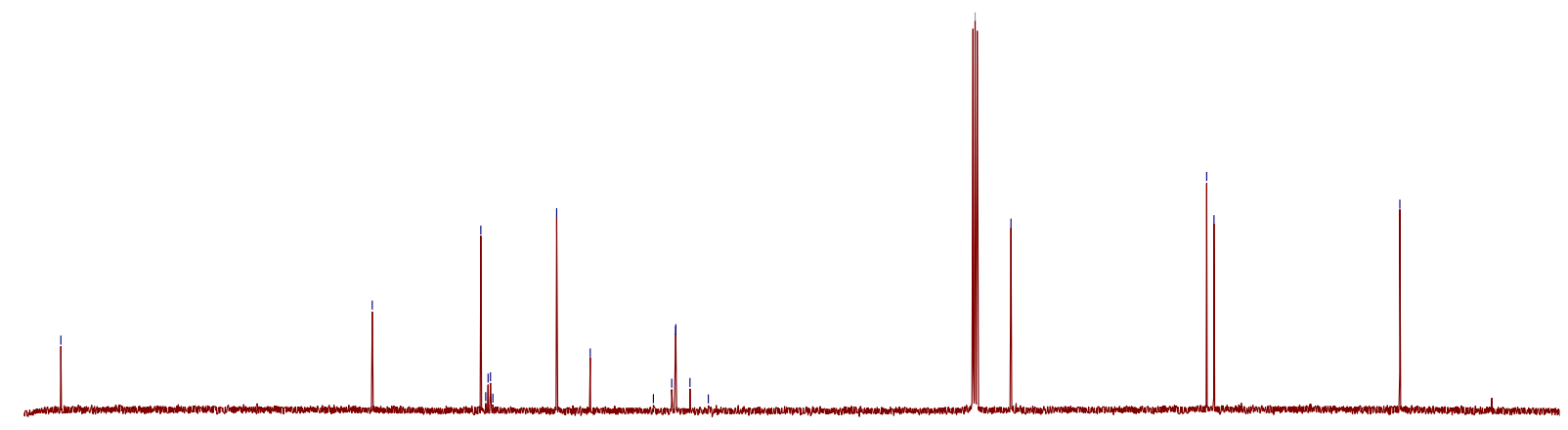



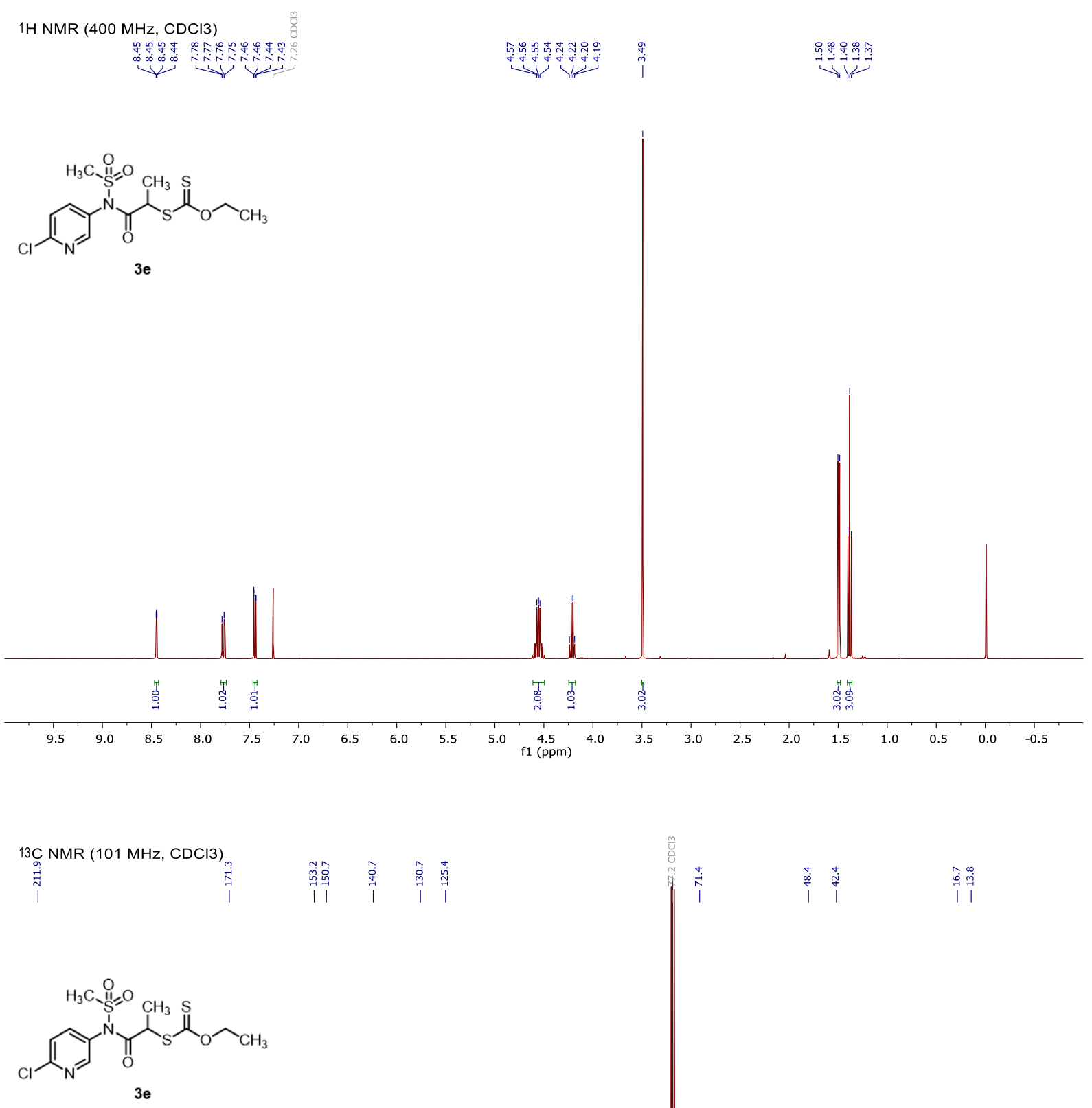

$3 e$

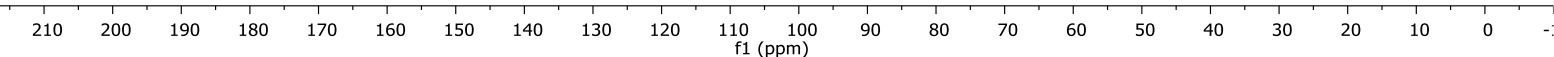



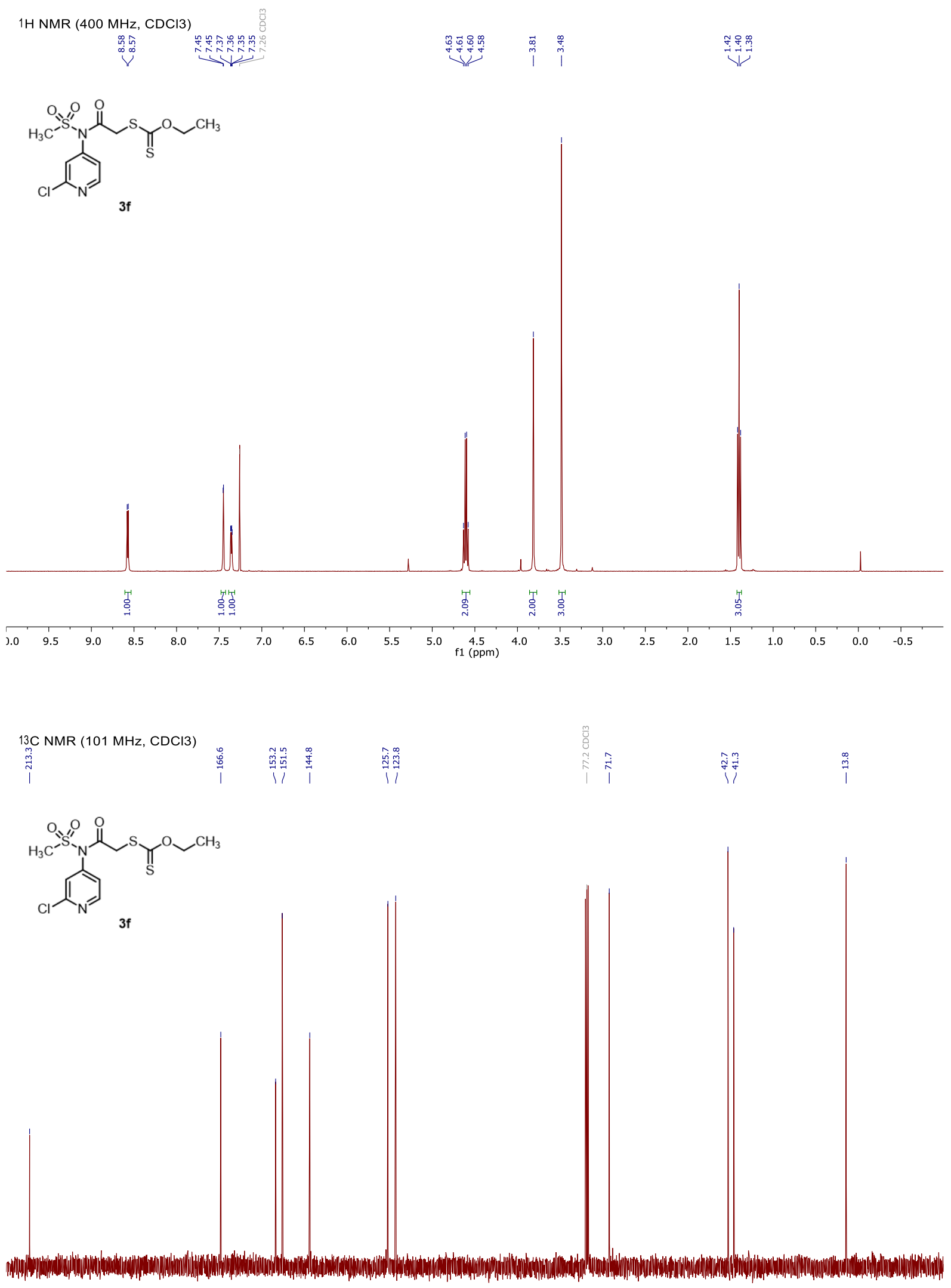

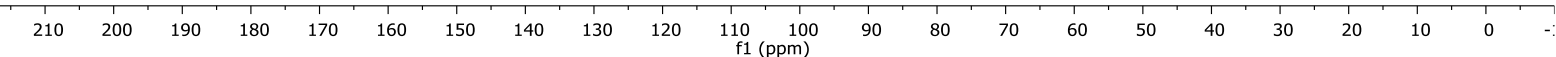


$1 \mathrm{H} \mathrm{NMR}(400 \mathrm{MHz}, \mathrm{CDCl} 3)$

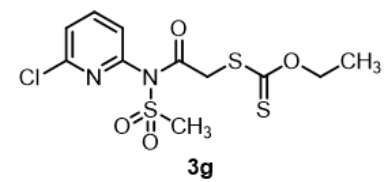

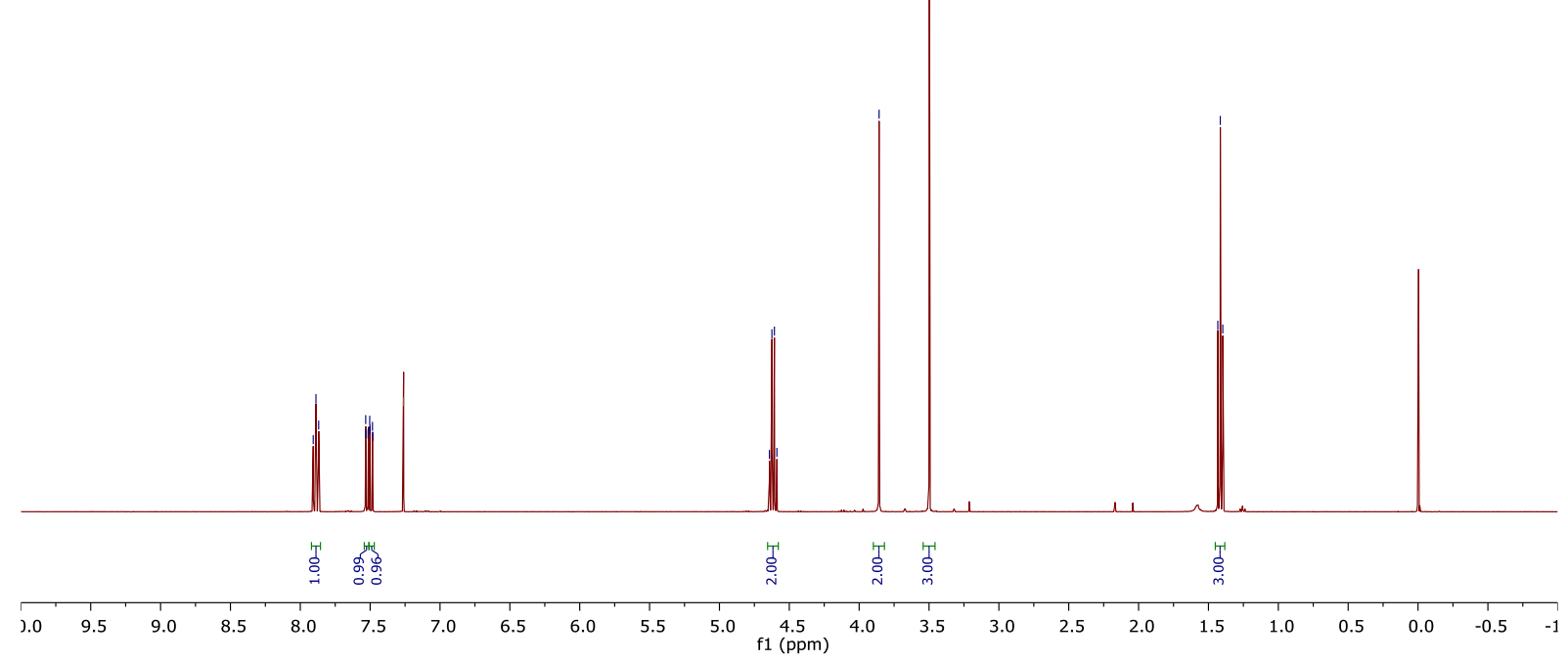

$$
\text { (101 MHz, CDCl3) }
$$

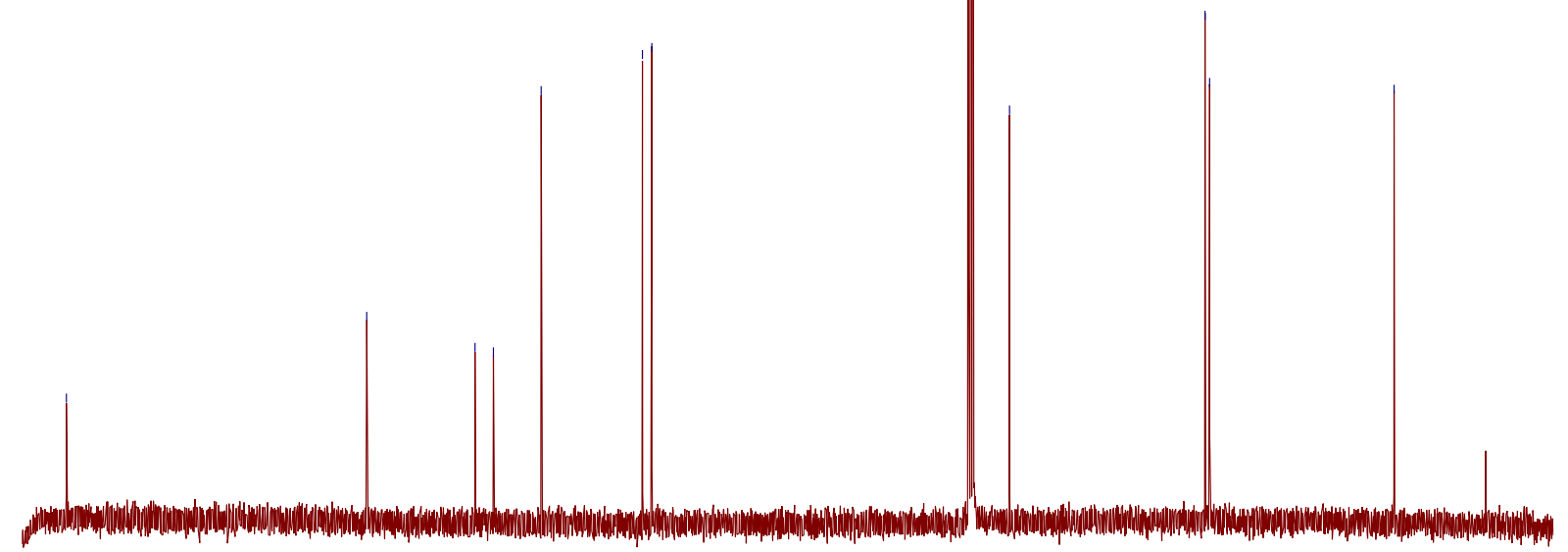

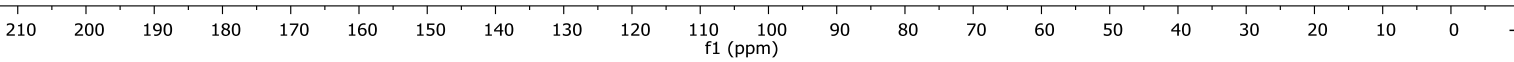



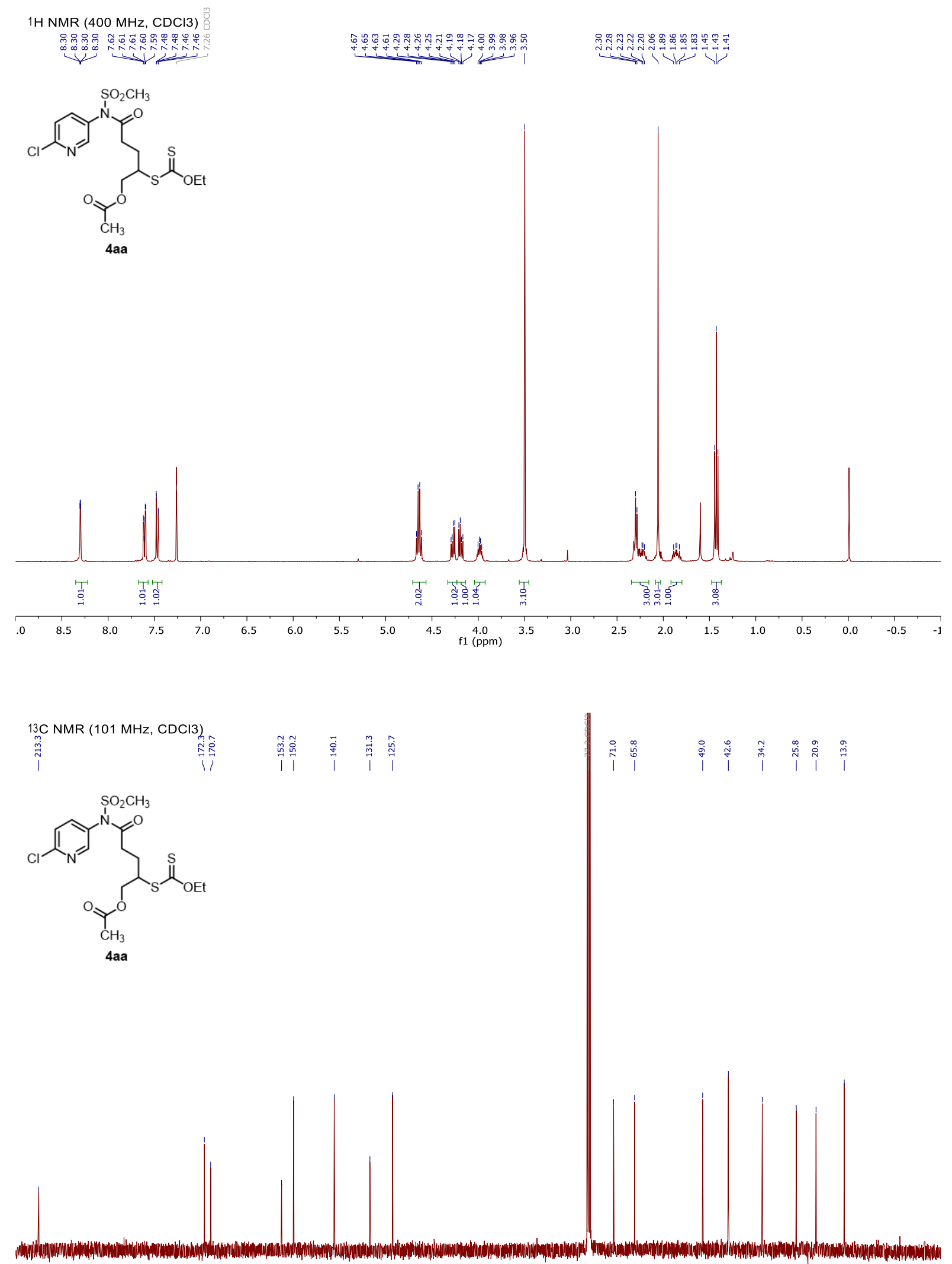

$\begin{array}{llllllllllllllllllllll}210 & 200 & 190 & 180 & 170 & 160 & 150 & 140 & 130 & 120 & \underset{\mathrm{f} 1(\mathrm{ppm})}{100} & 90 & 80 & 70 & 60 & 50 & 40 & 30 & 20 & 10 & 0 & -:\end{array}$ 


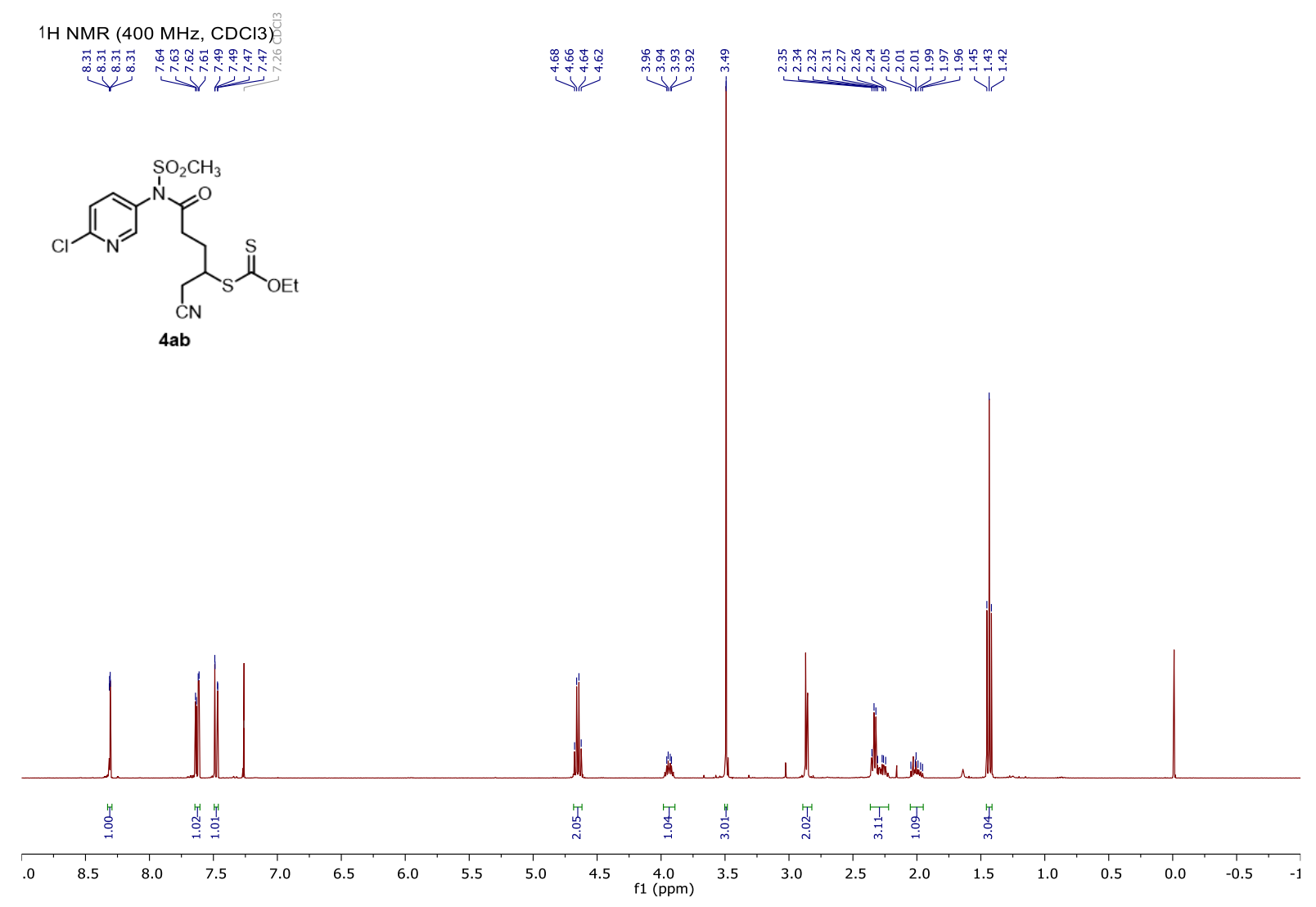

13C NMR (101 MHz, CDCl3)<smiles>CCCC(CCC(=O)N(C)S(=O)(=O)c1ccc(Cl)nc1)SC(=S)OCC</smiles>

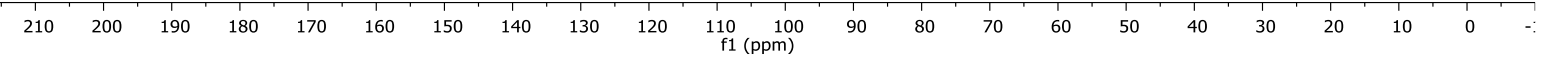



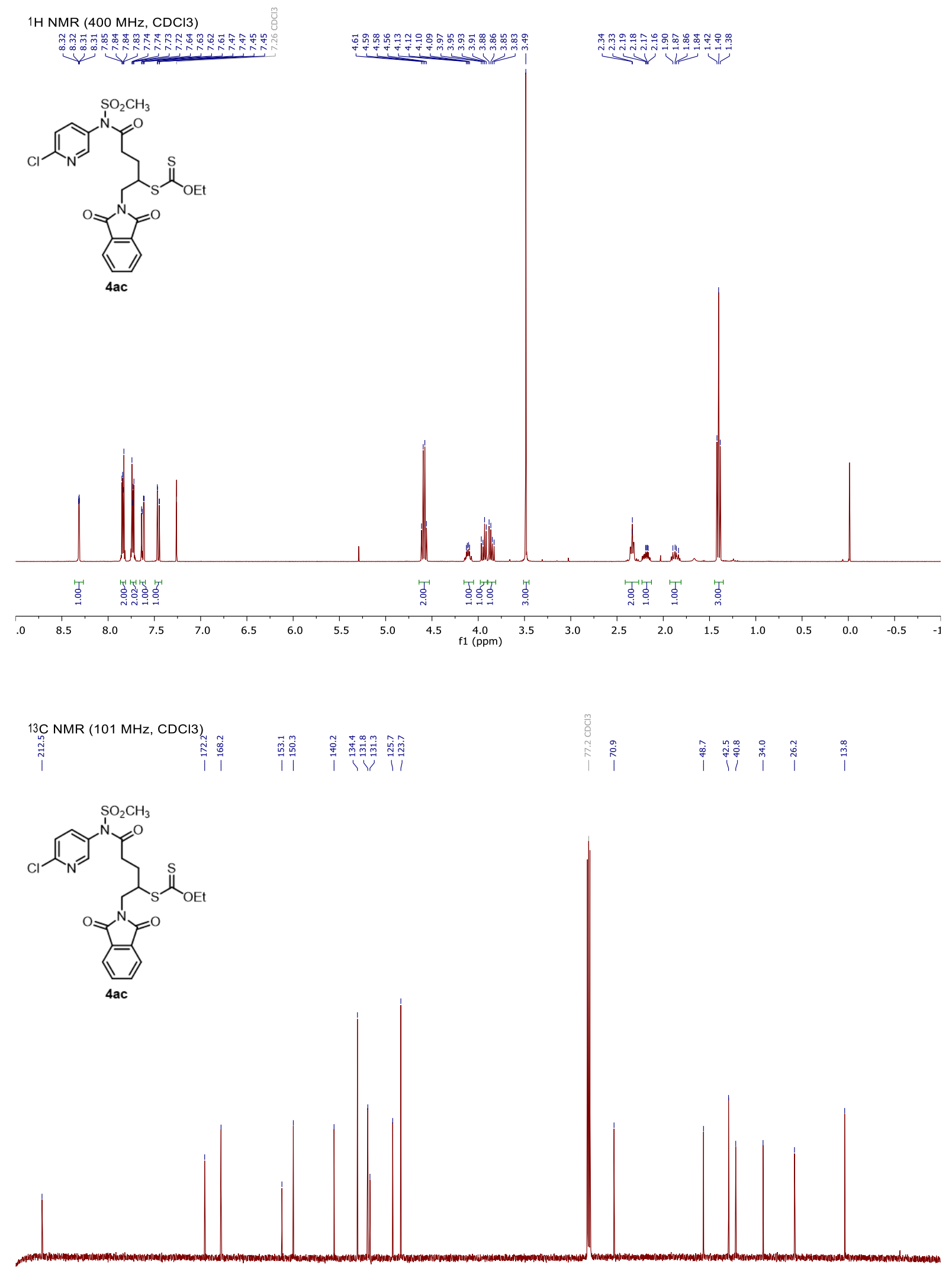

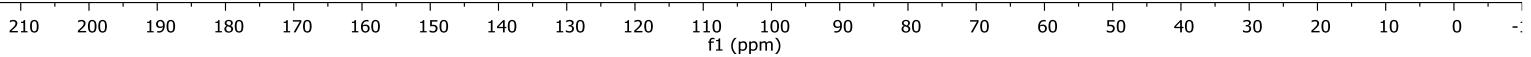



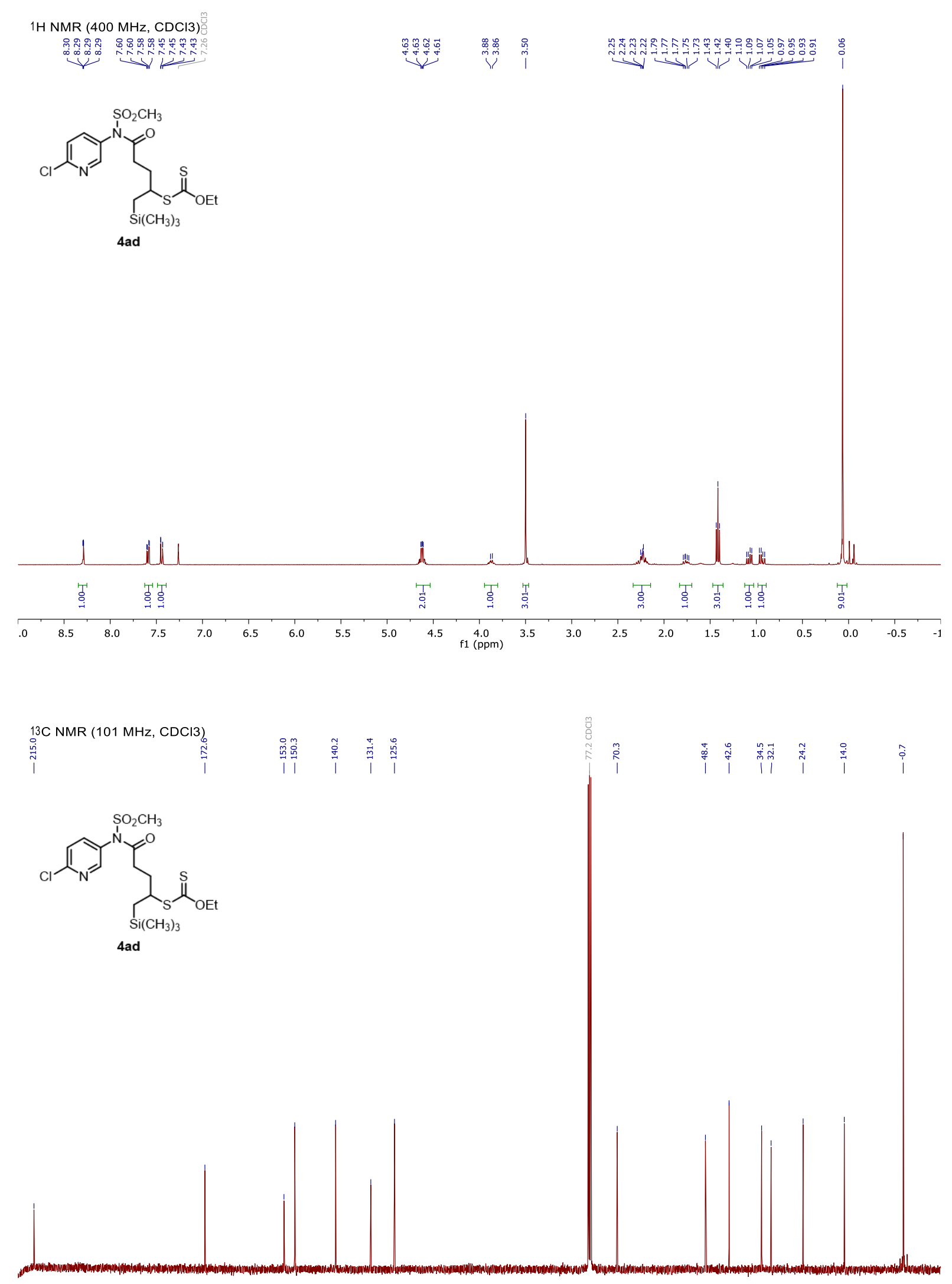

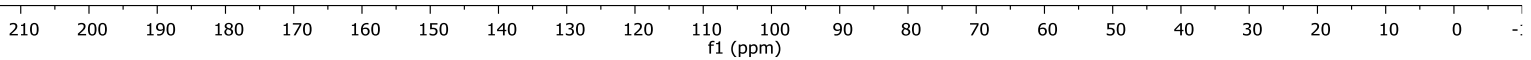



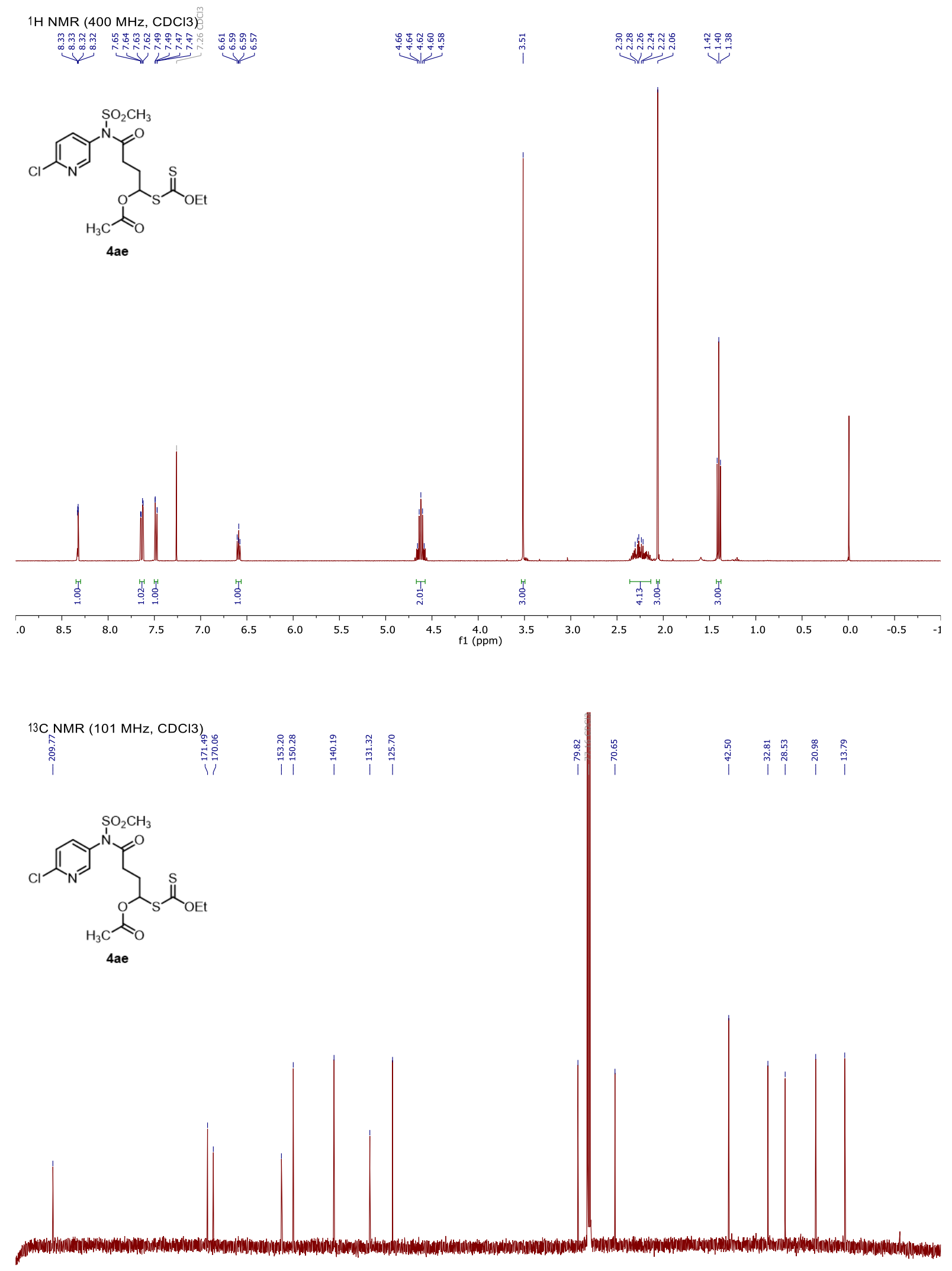

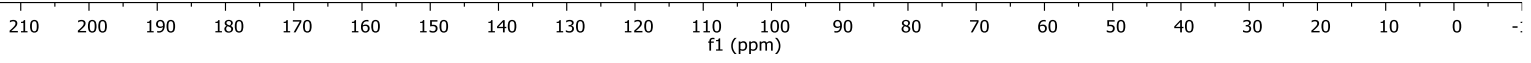



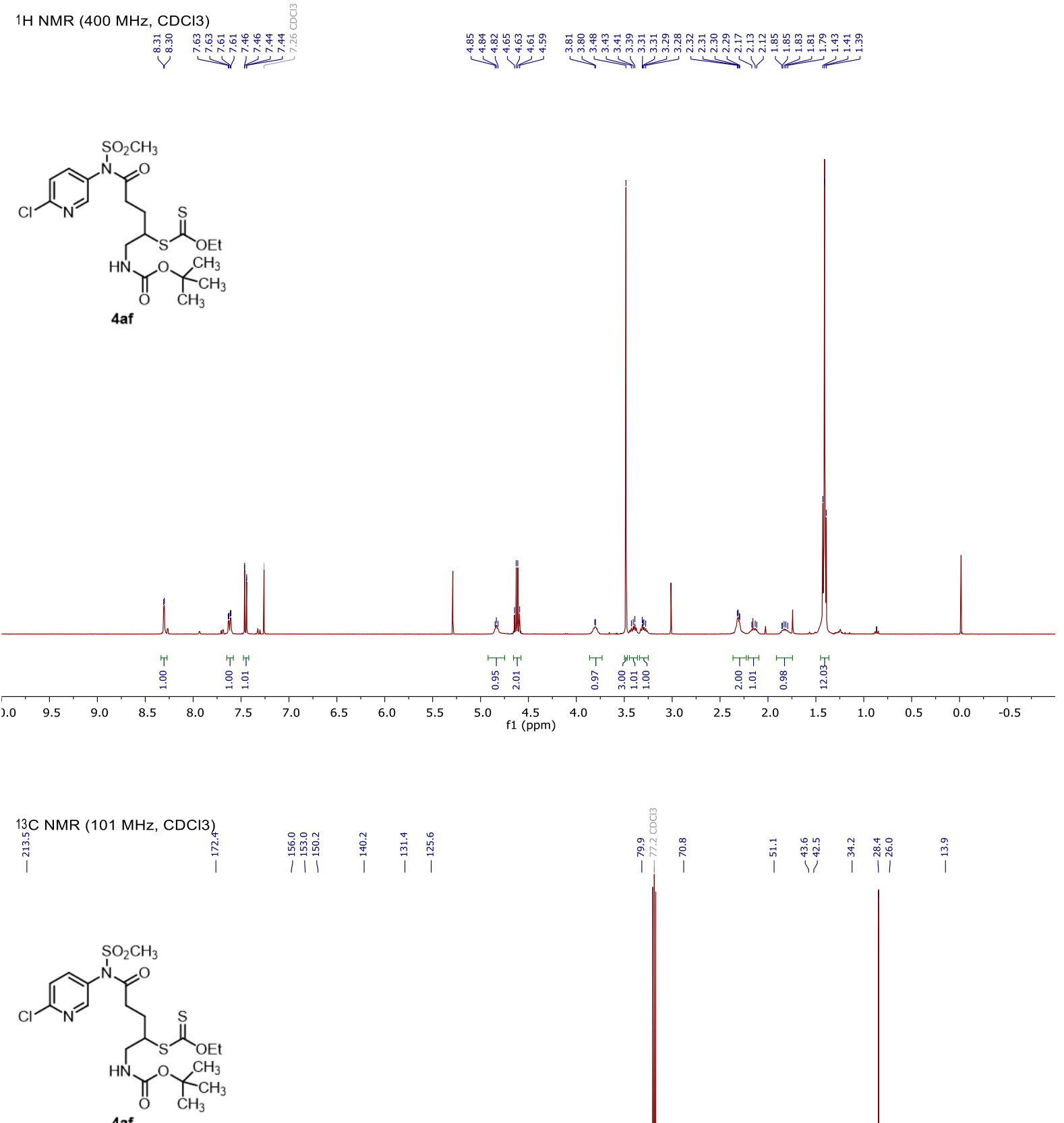

Th Th

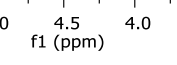

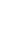



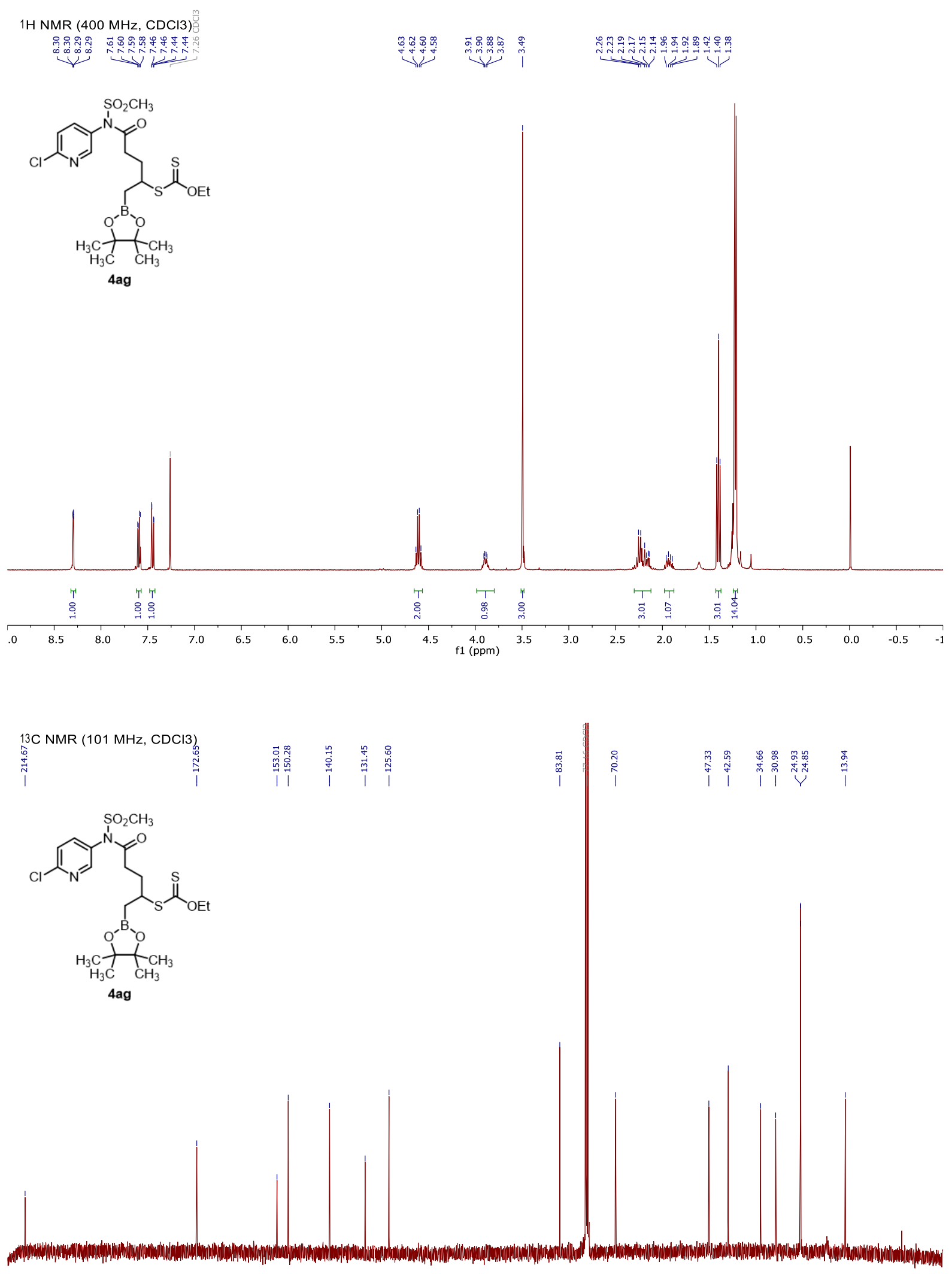

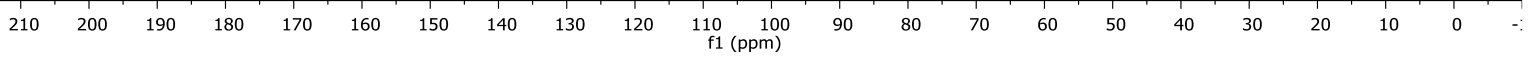




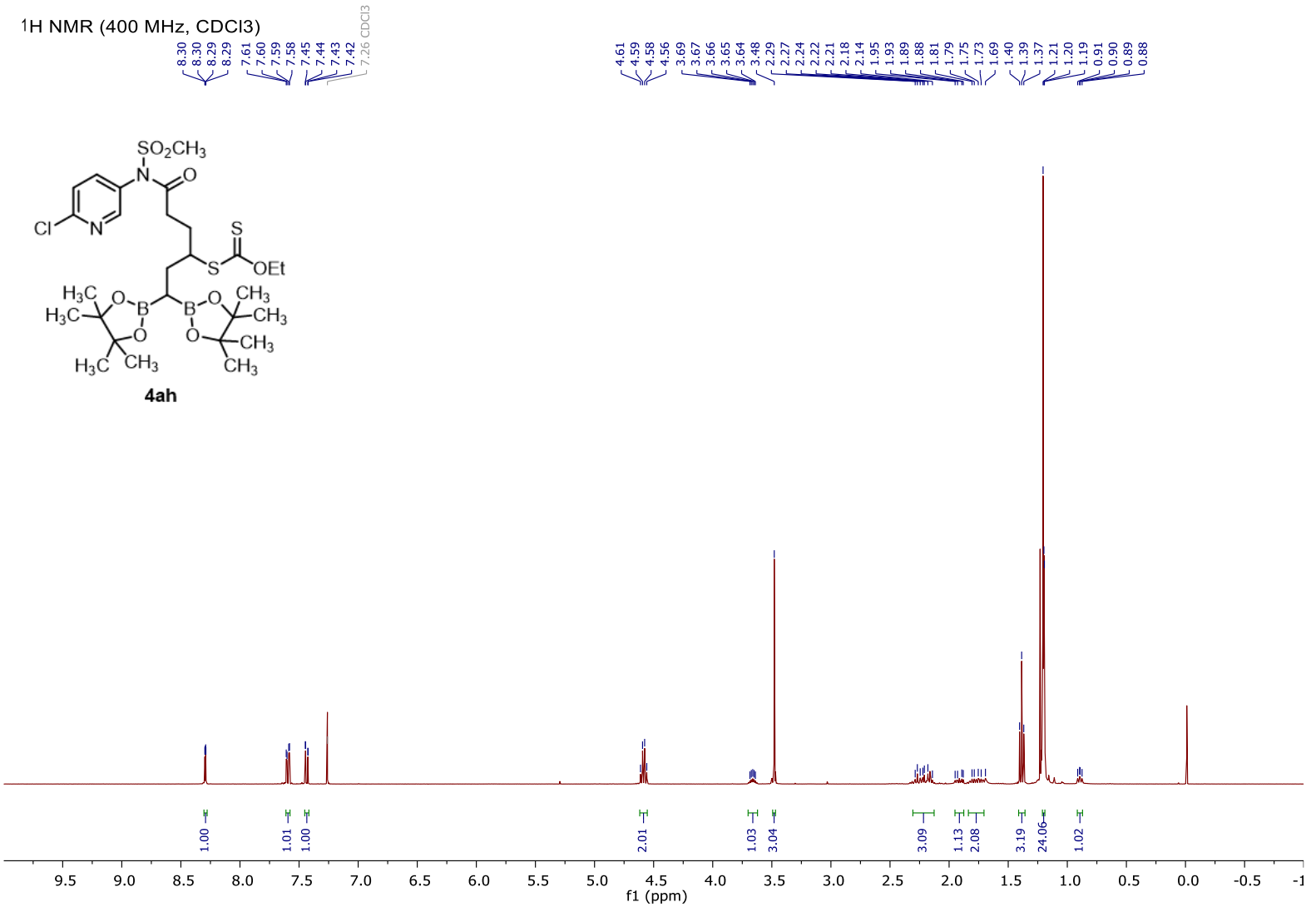

13C NMR (101 MHz, CDCl3)<smiles>CCOC(=S)SC(CCC(=O)N(C)c1ccc(Cl)nc1)CC1OB2OC(C)(C)B1OC(C)(C)C(C)(C)O2</smiles>

4ah

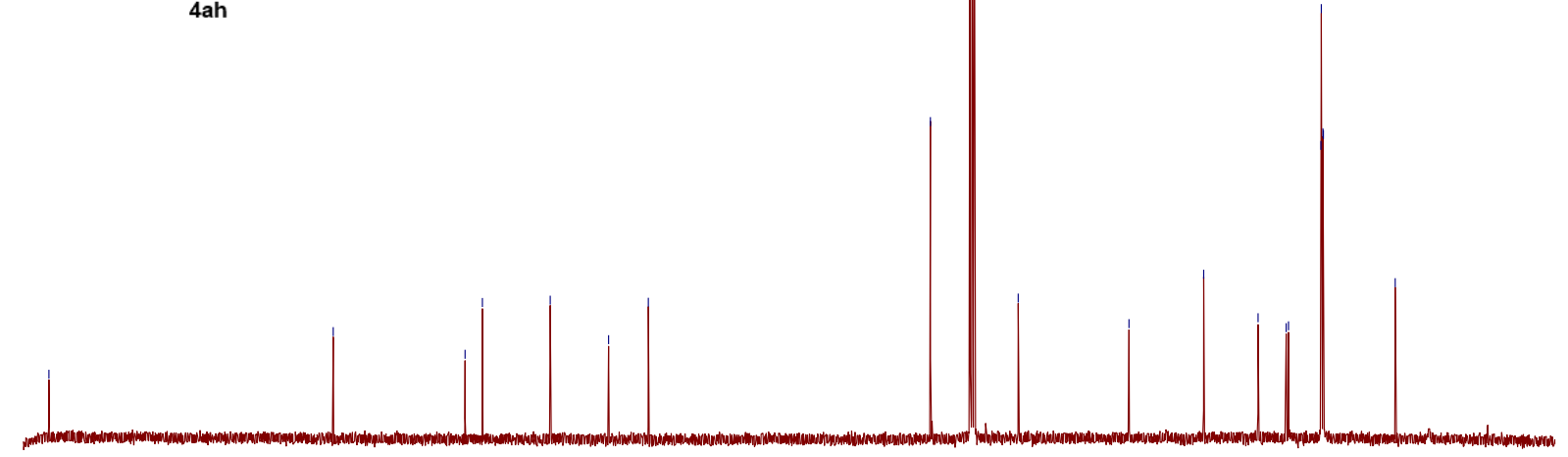

$\begin{array}{llllllllllllllllllllllll}210 & 200 & 190 & 180 & 170 & 160 & 150 & 140 & 130 & 120 & \begin{array}{c}110 \\ \mathrm{f} 1(\mathrm{ppm})\end{array} & 90 & 80 & 70 & 60 & 50 & 40 & 30 & 20 & 10 & 0 & -:\end{array}$ 

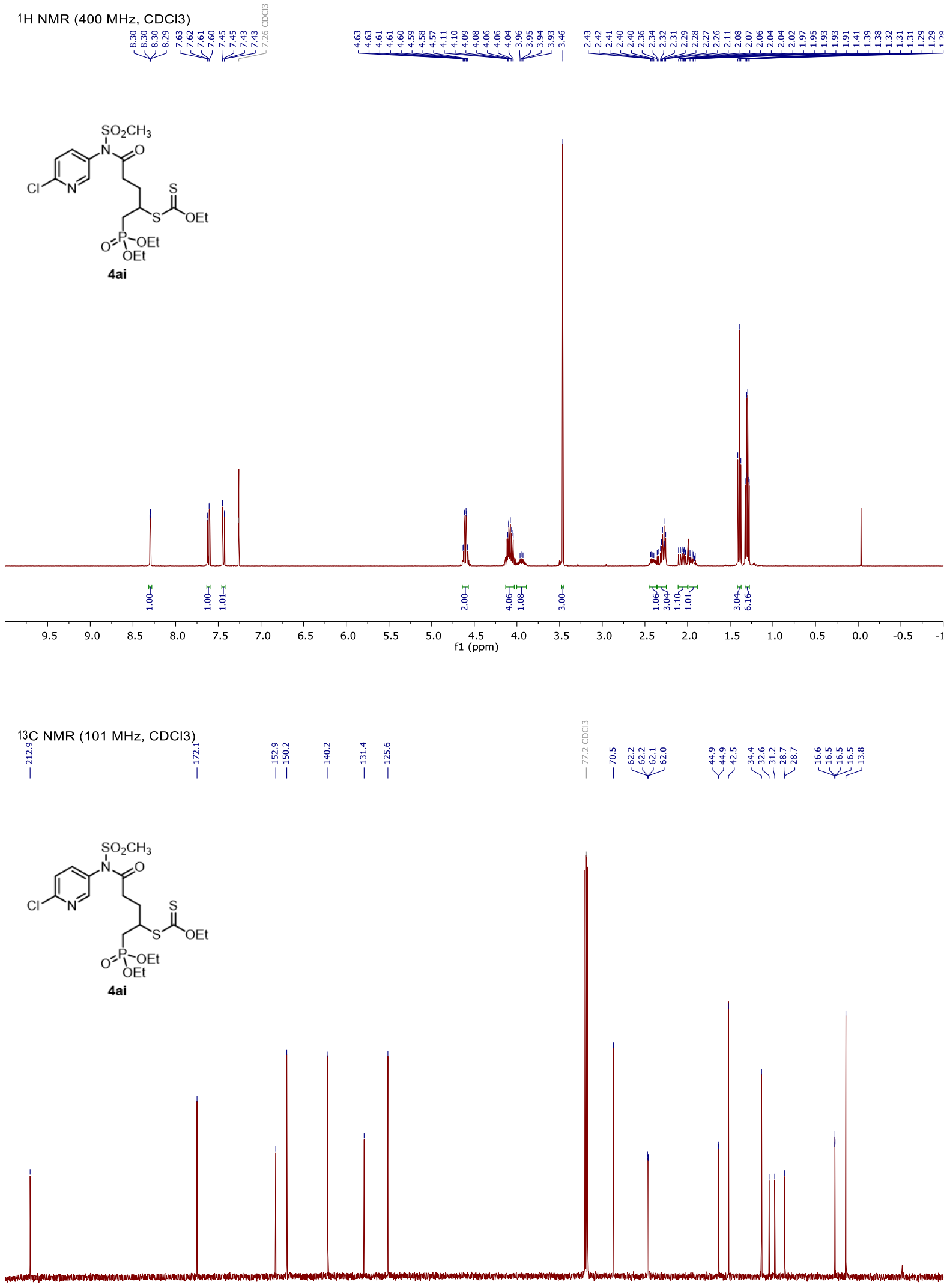

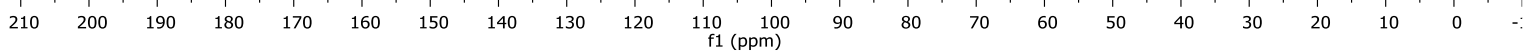



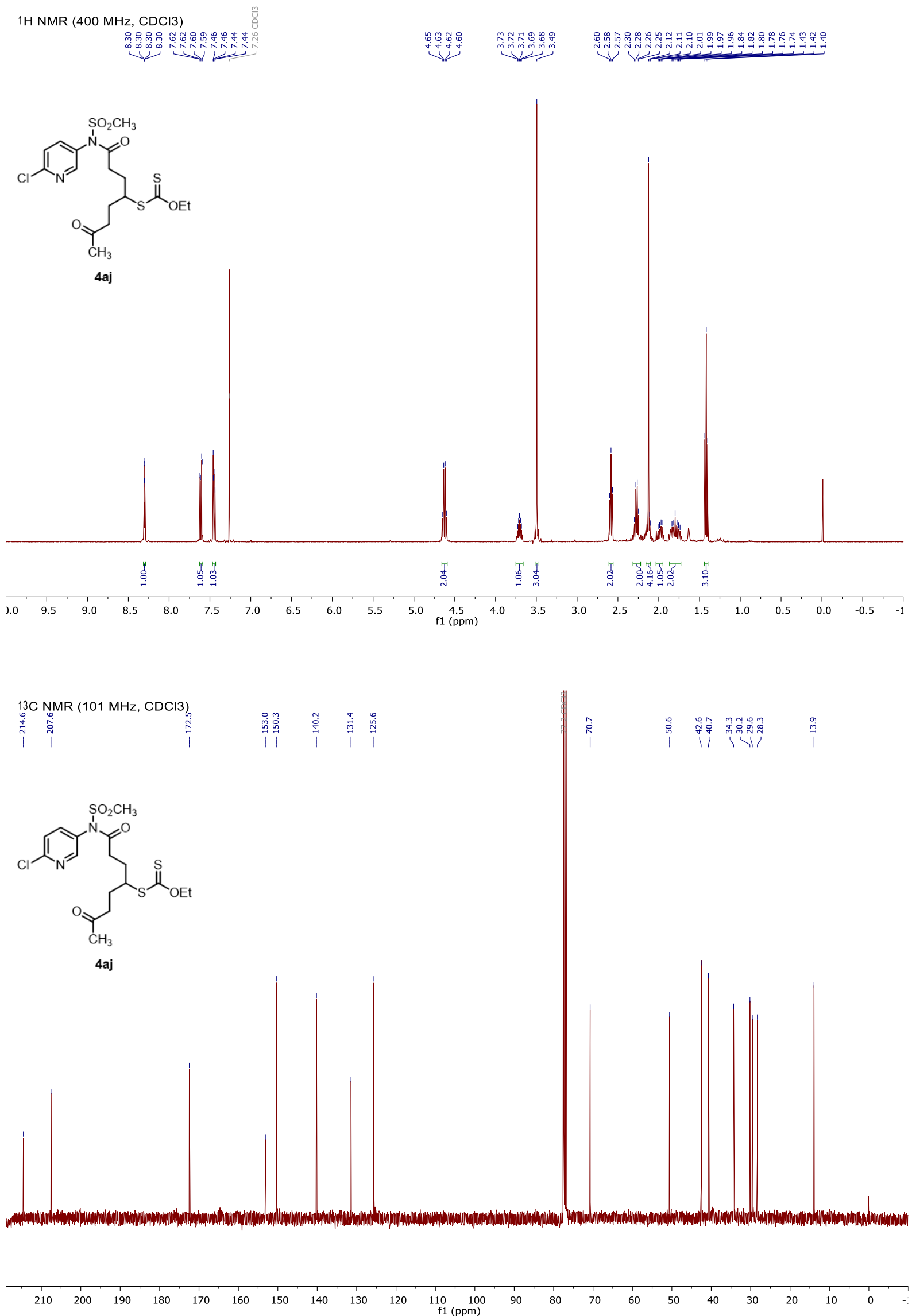

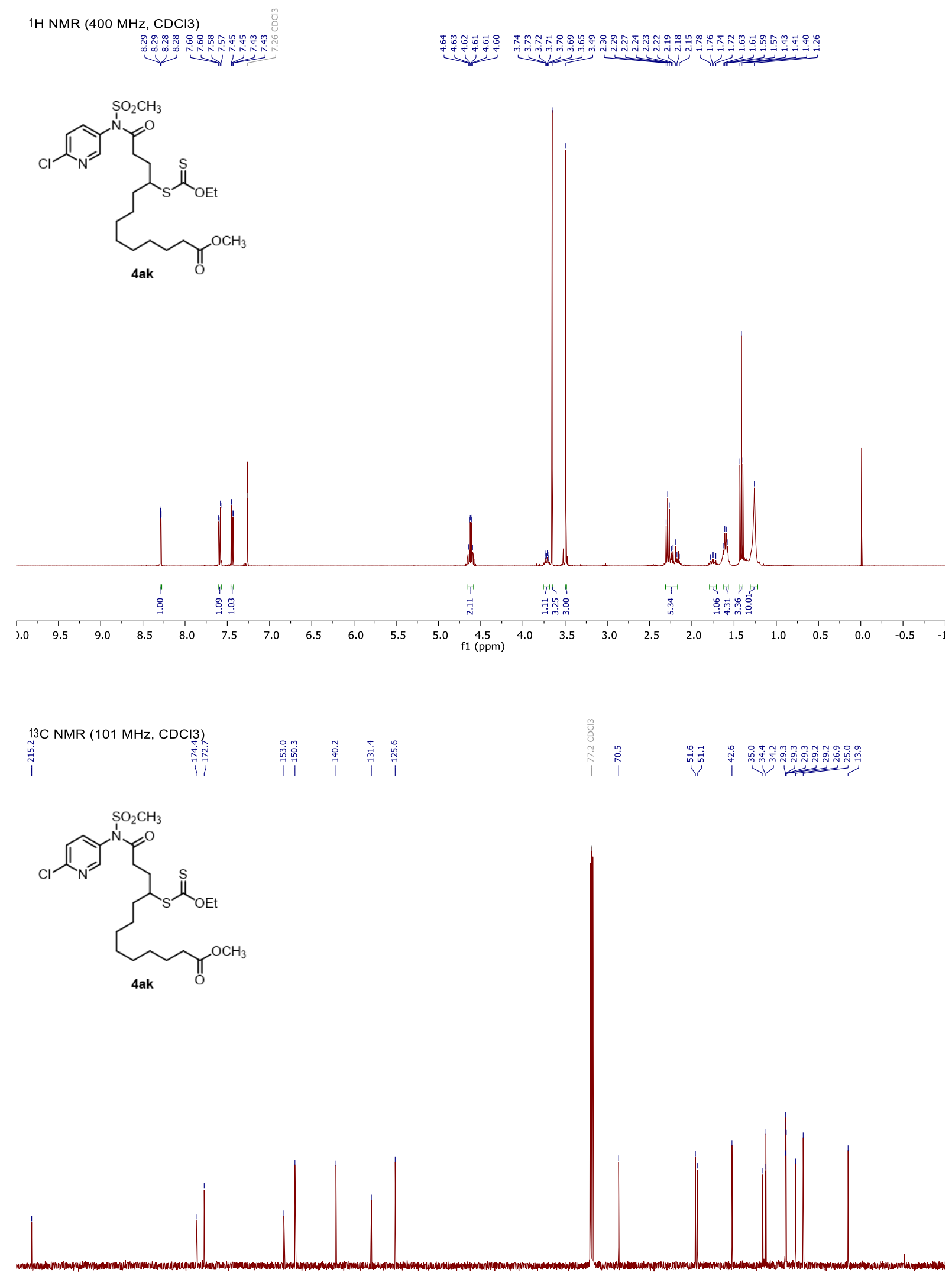

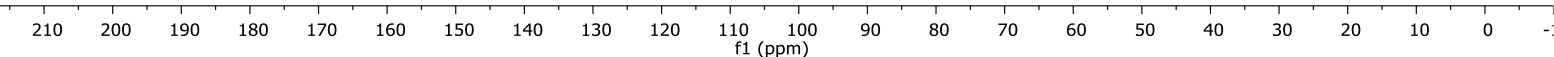




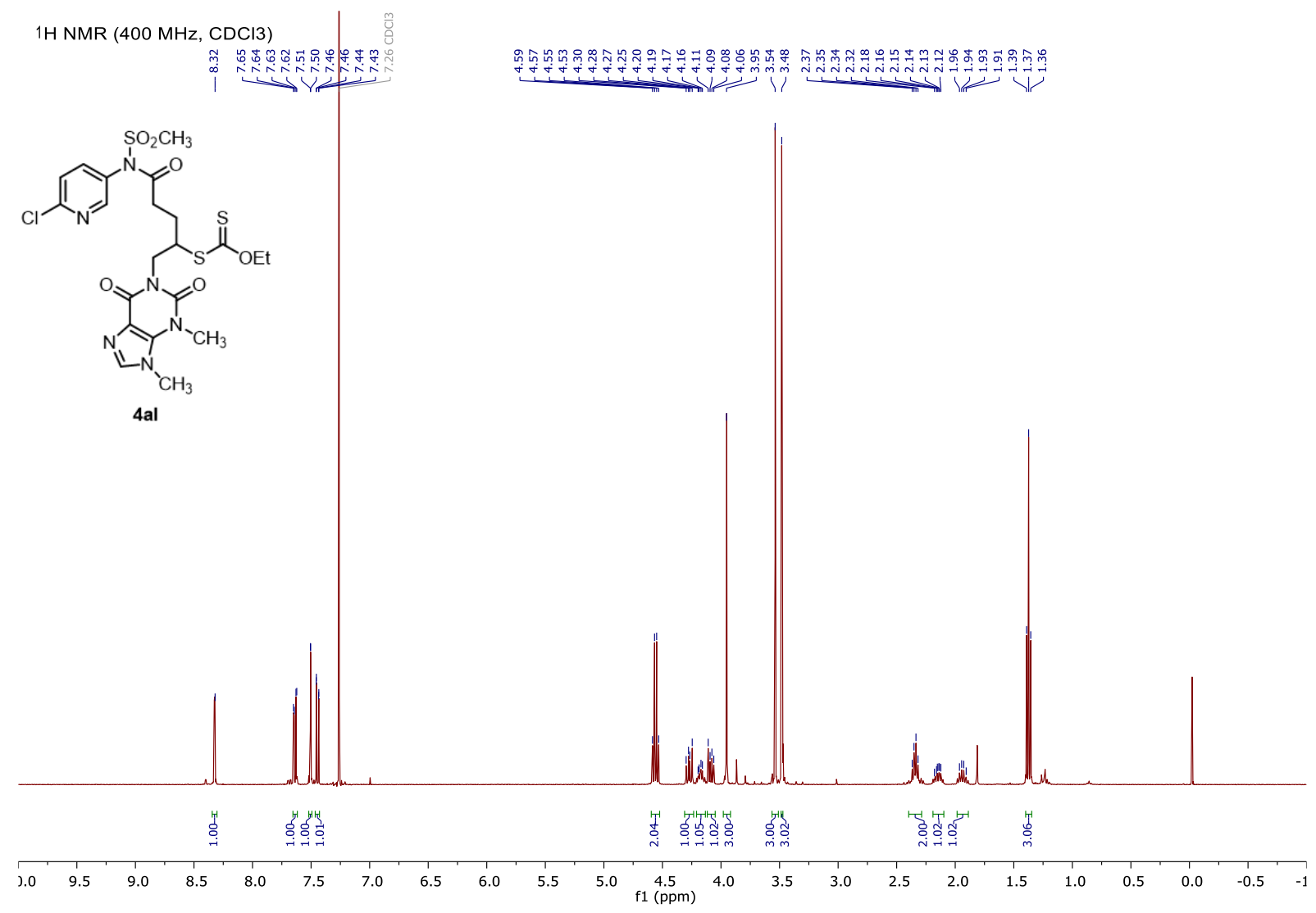

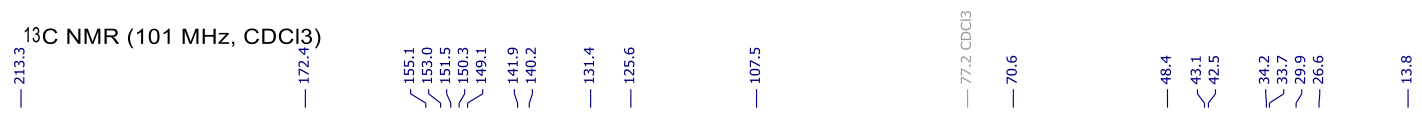

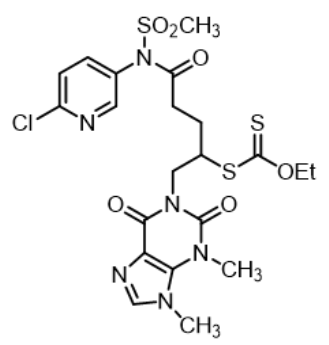

4al

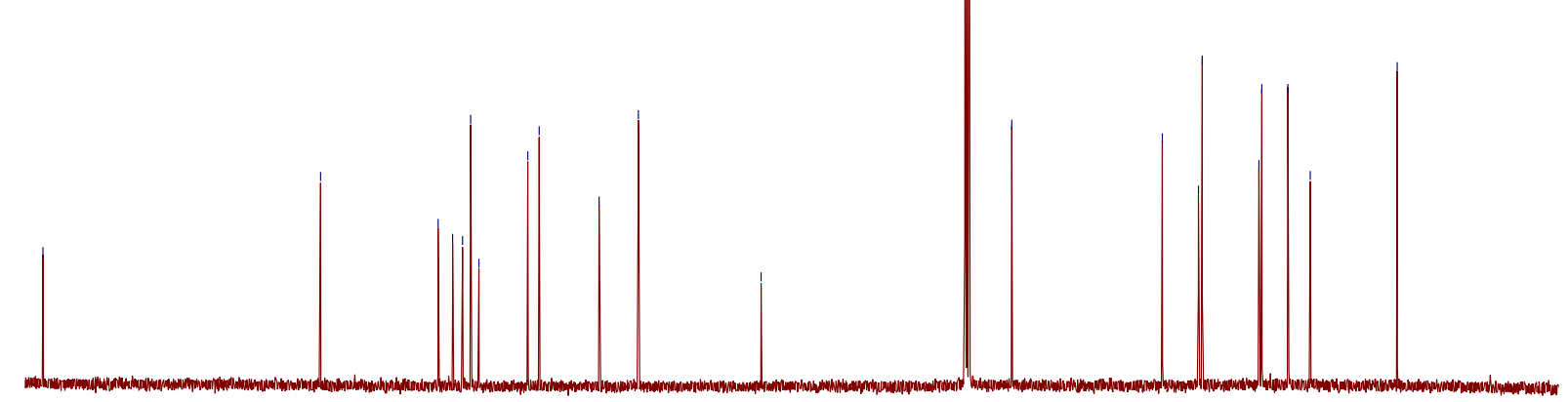

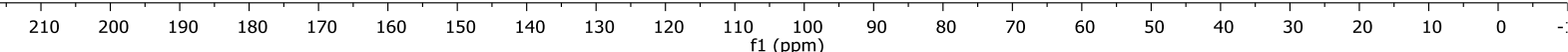



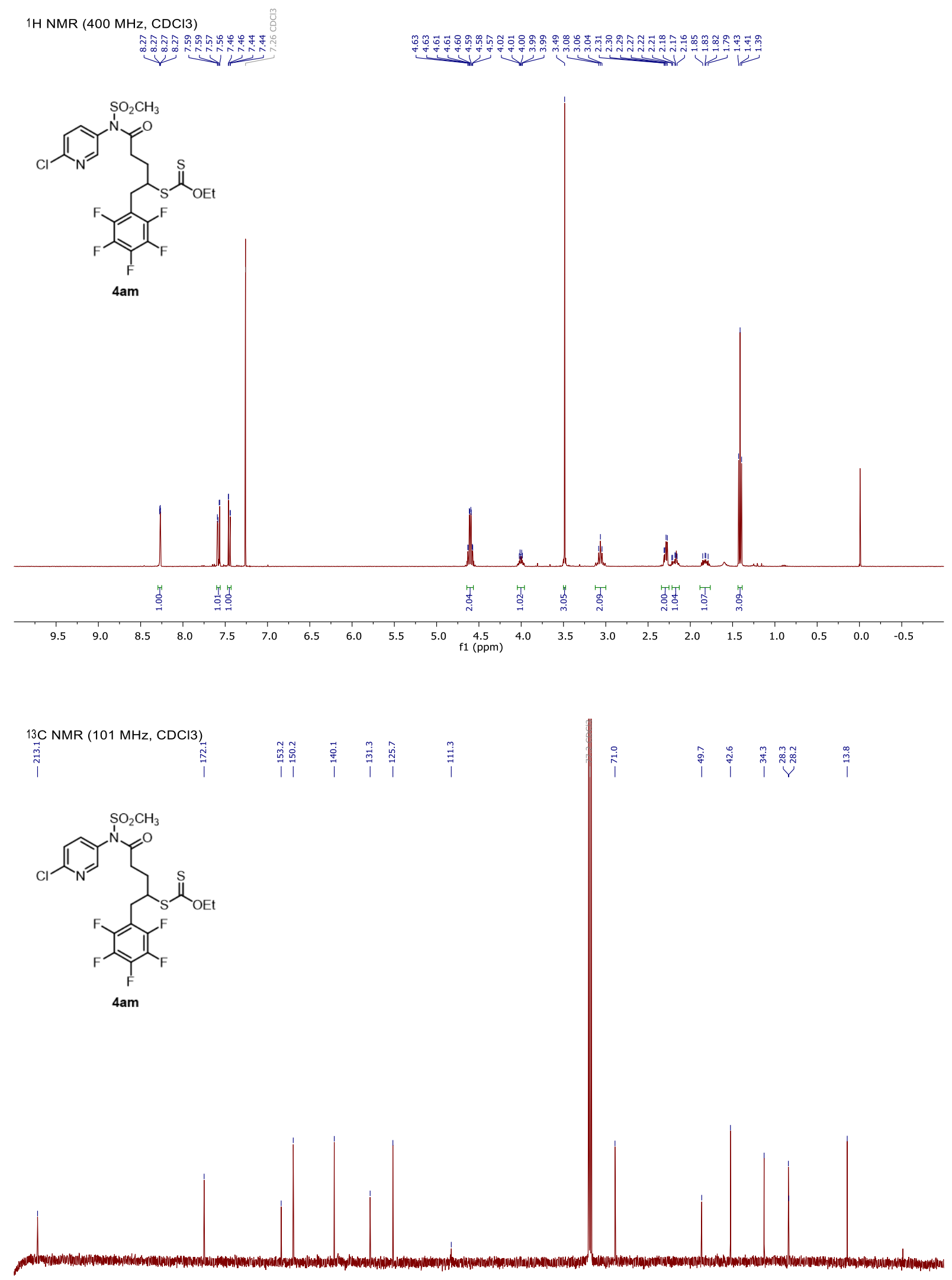

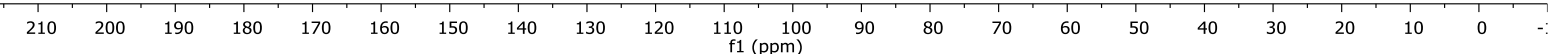


1H NMR (400 MHz, CDCl3)

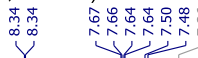

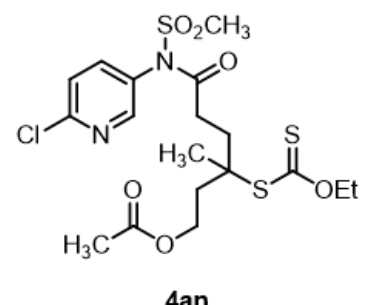

4an

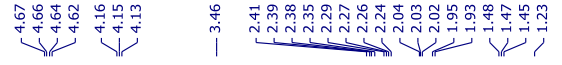

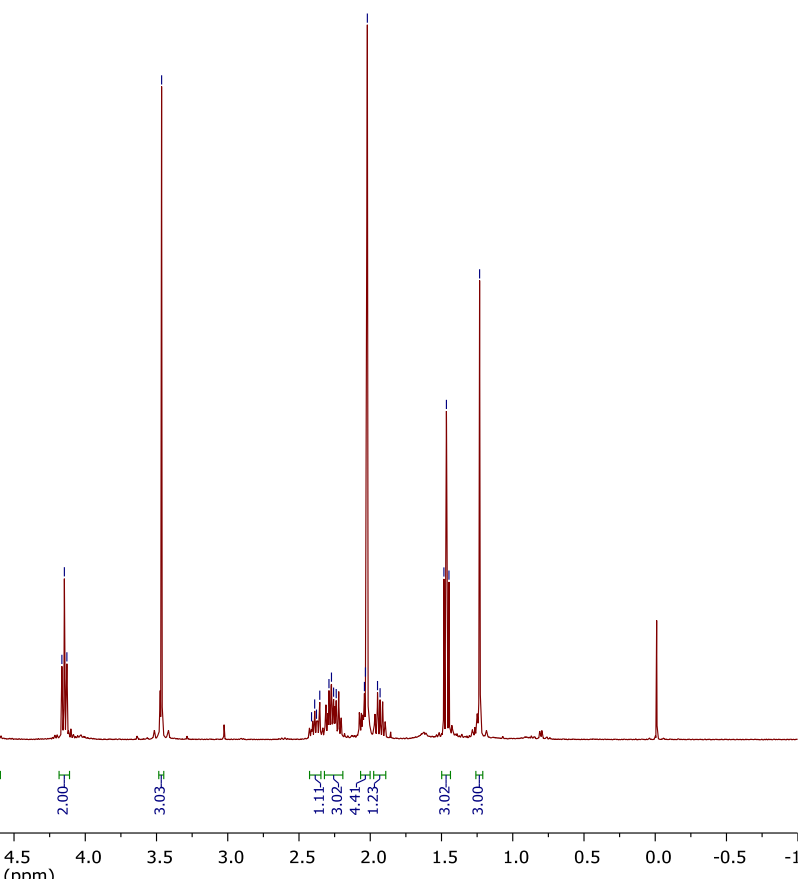

13С NMR (101 MHz, CDCl3)

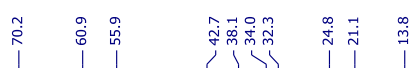<smiles>CCOC(=S)SC(C)(CCOC(C)=O)CCC(=O)N(C(C)=O)c1ccc(Cl)nc1</smiles>

4 an

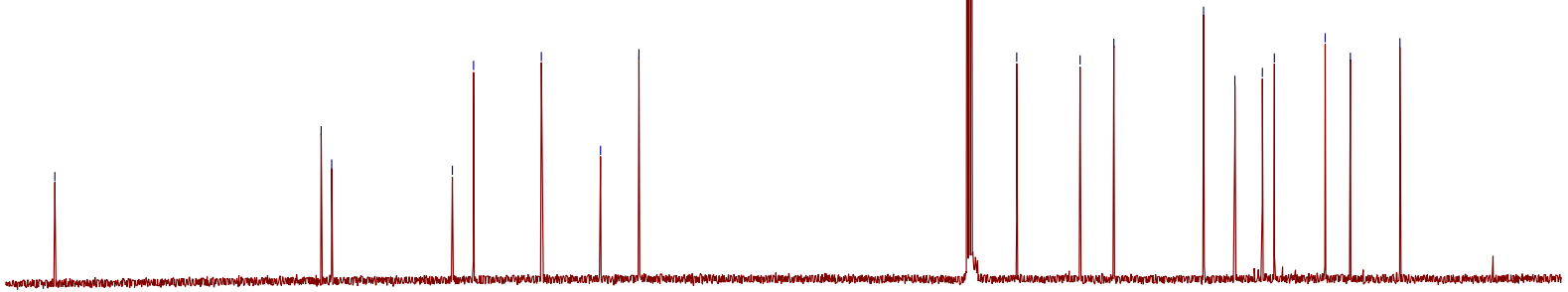

$\begin{array}{llllllllllll}210 & 200 & 190 & 180 & 170 & 160 & 150 & 140 & 130 & 120 & 110 & 100 \\ & & & & & & & & & & \end{array}$ 

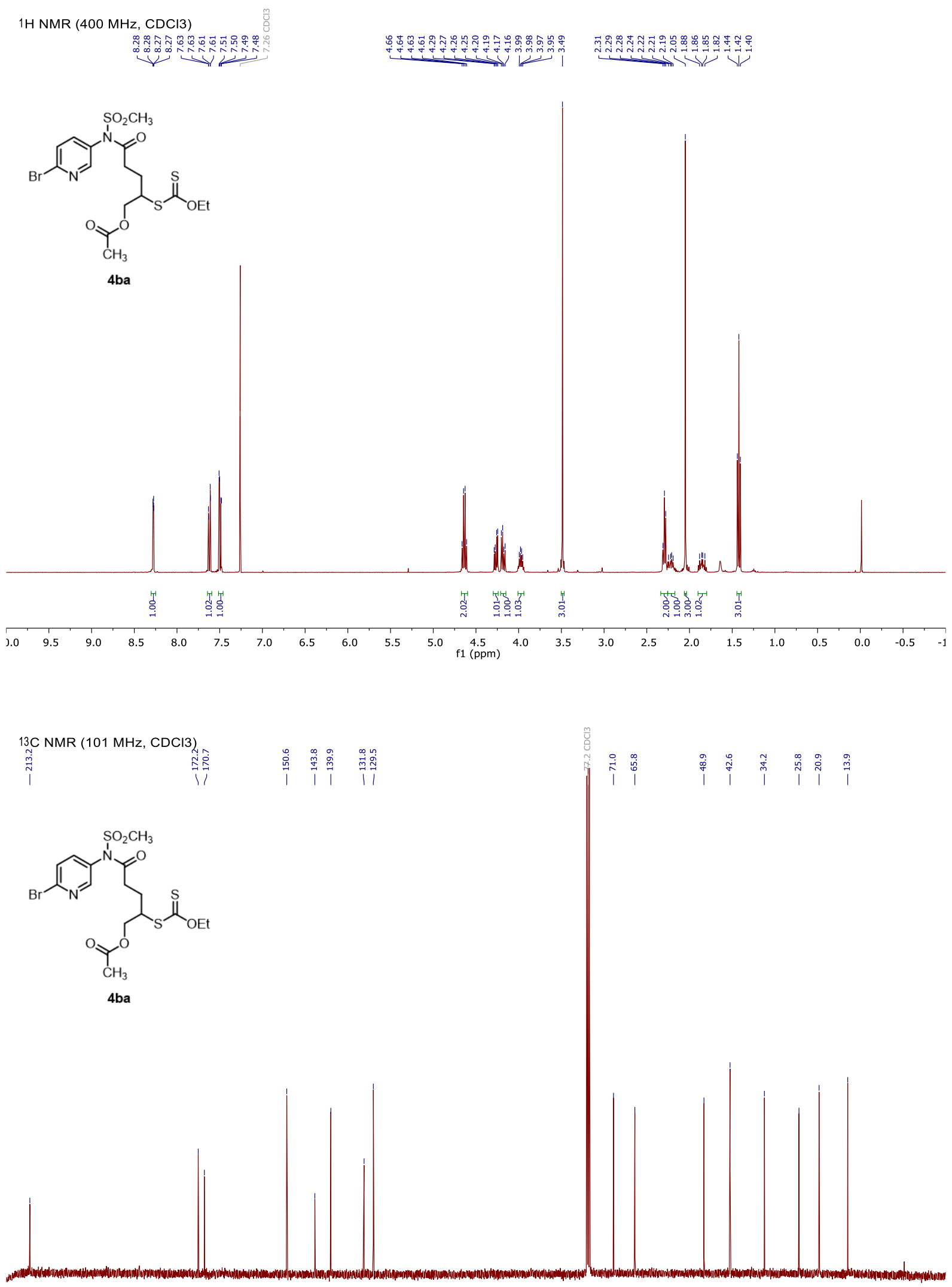

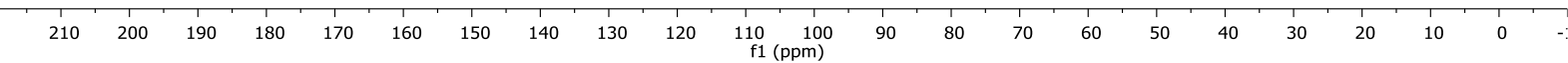




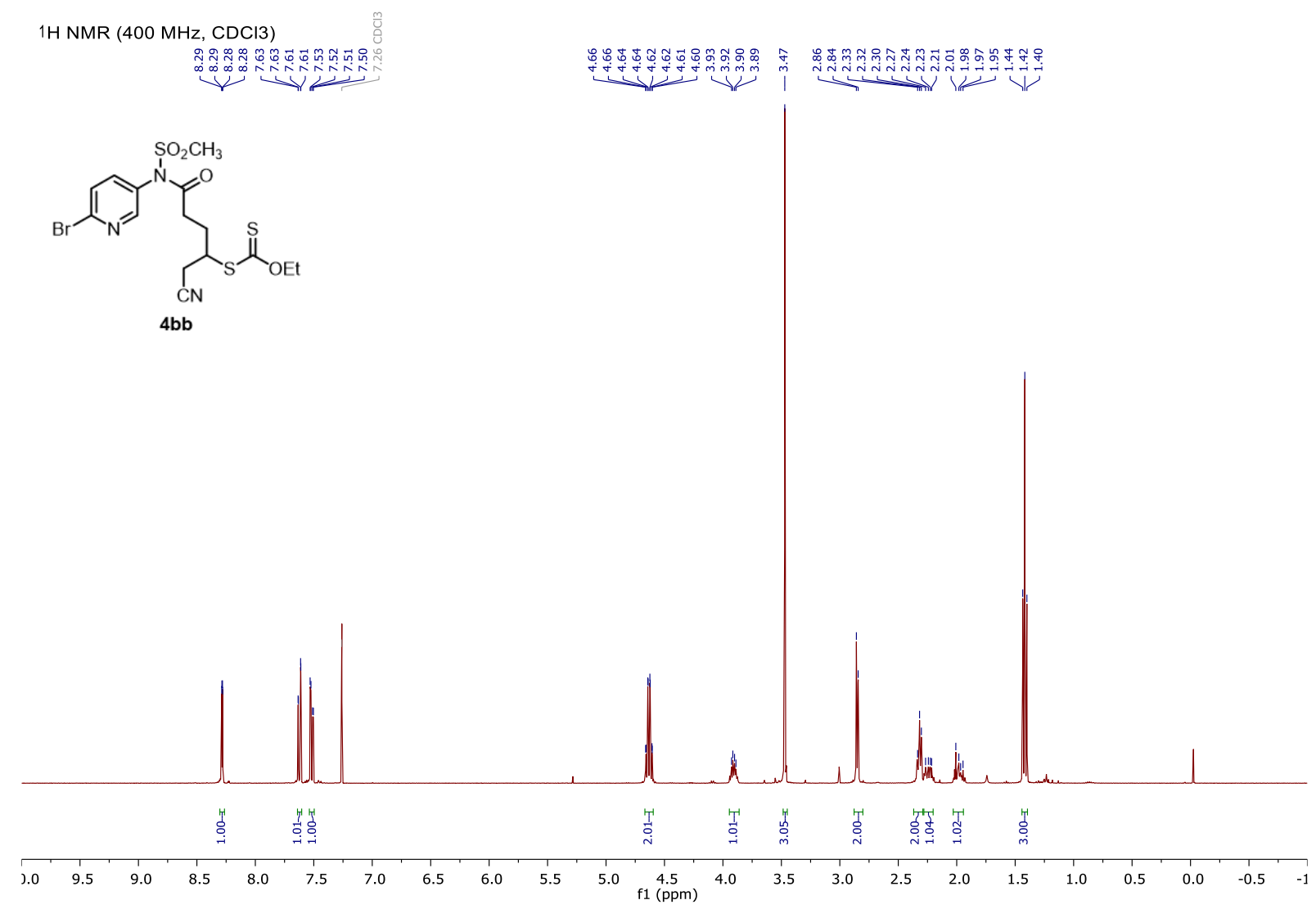

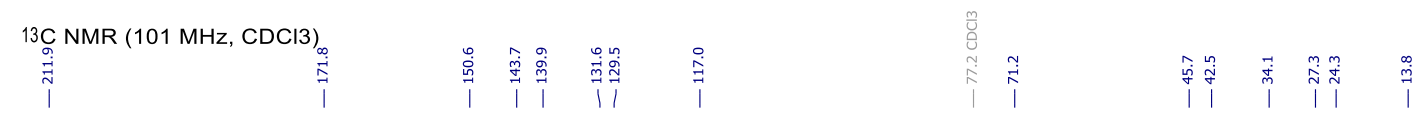<smiles>CCOC(=S)SC(CCC(=O)N(C)c1ccc(Br)nc1)C(=O)N[N+](=O)[O-]</smiles>

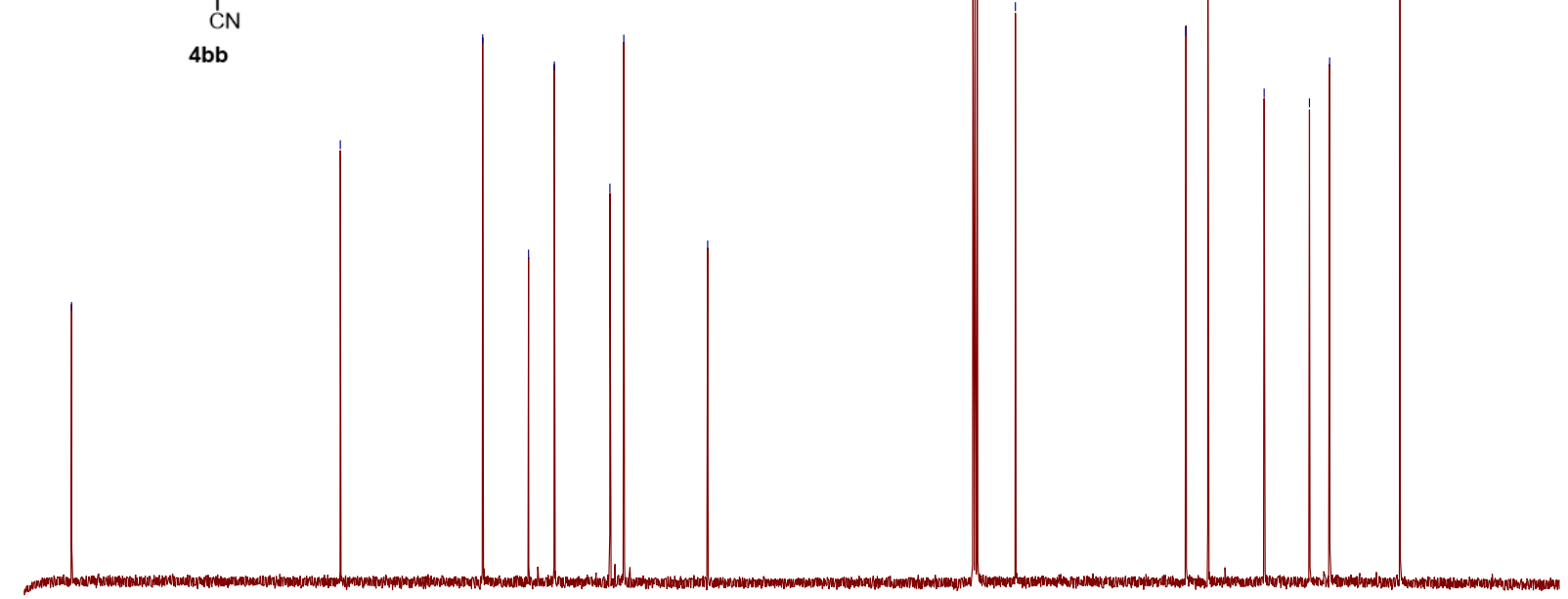

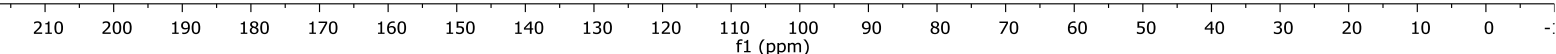




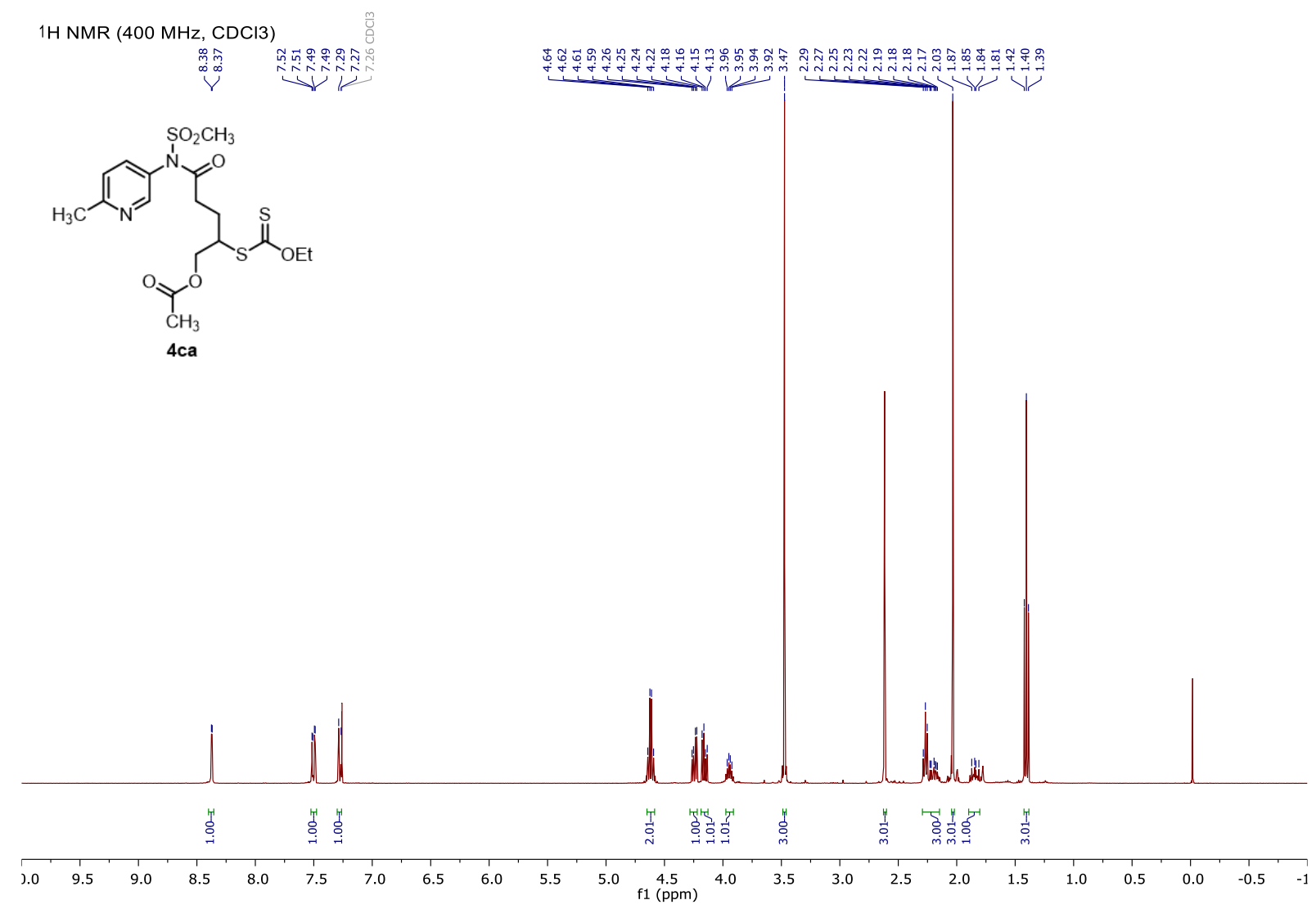

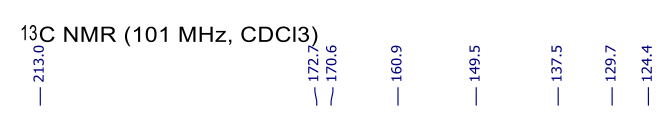

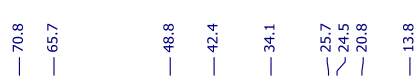<smiles>CCOC(=O)SC(CCC(=O)N(C)c1ccc(C)nc1)COC(C)=O</smiles>

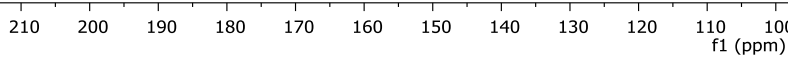



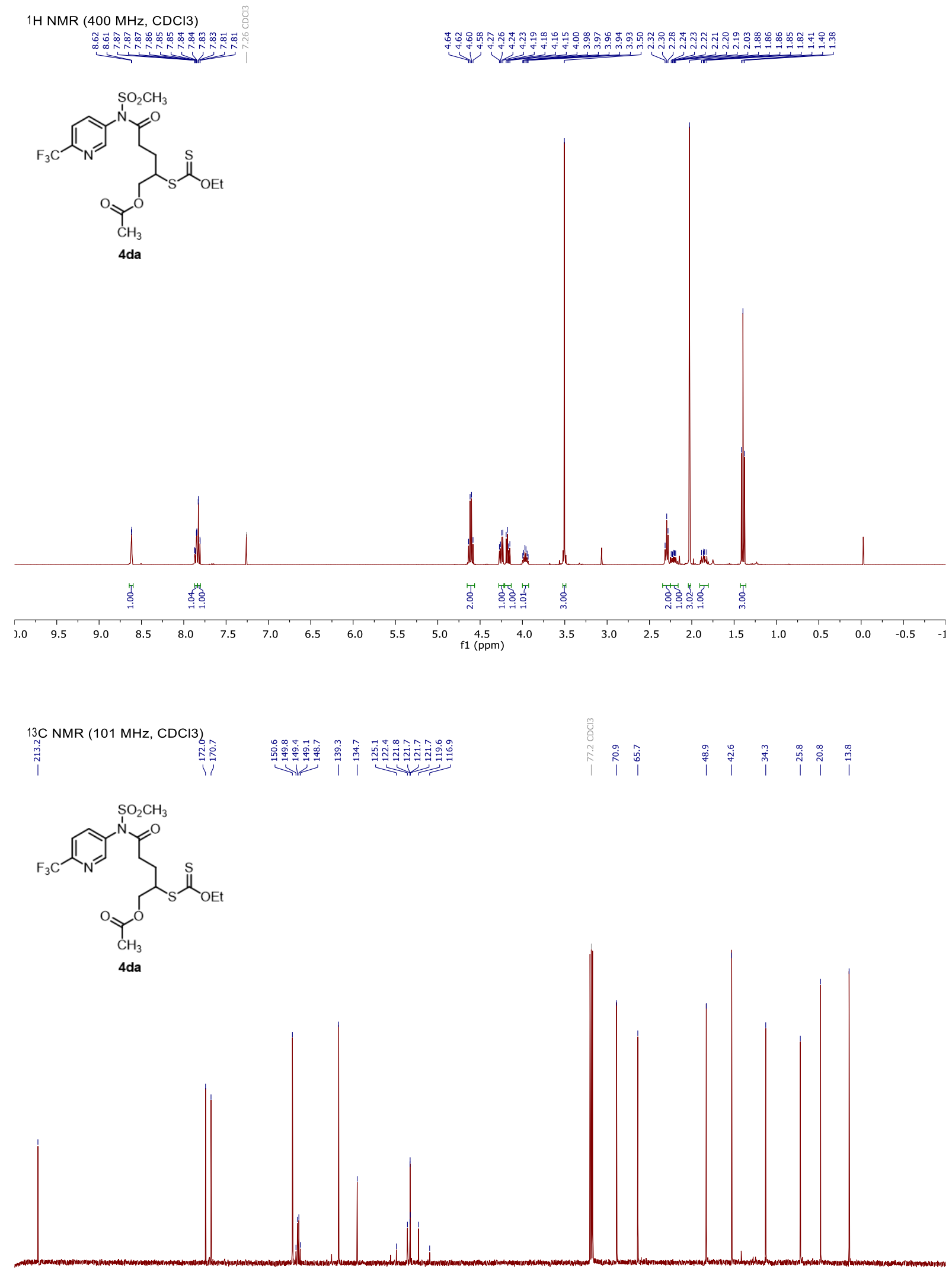

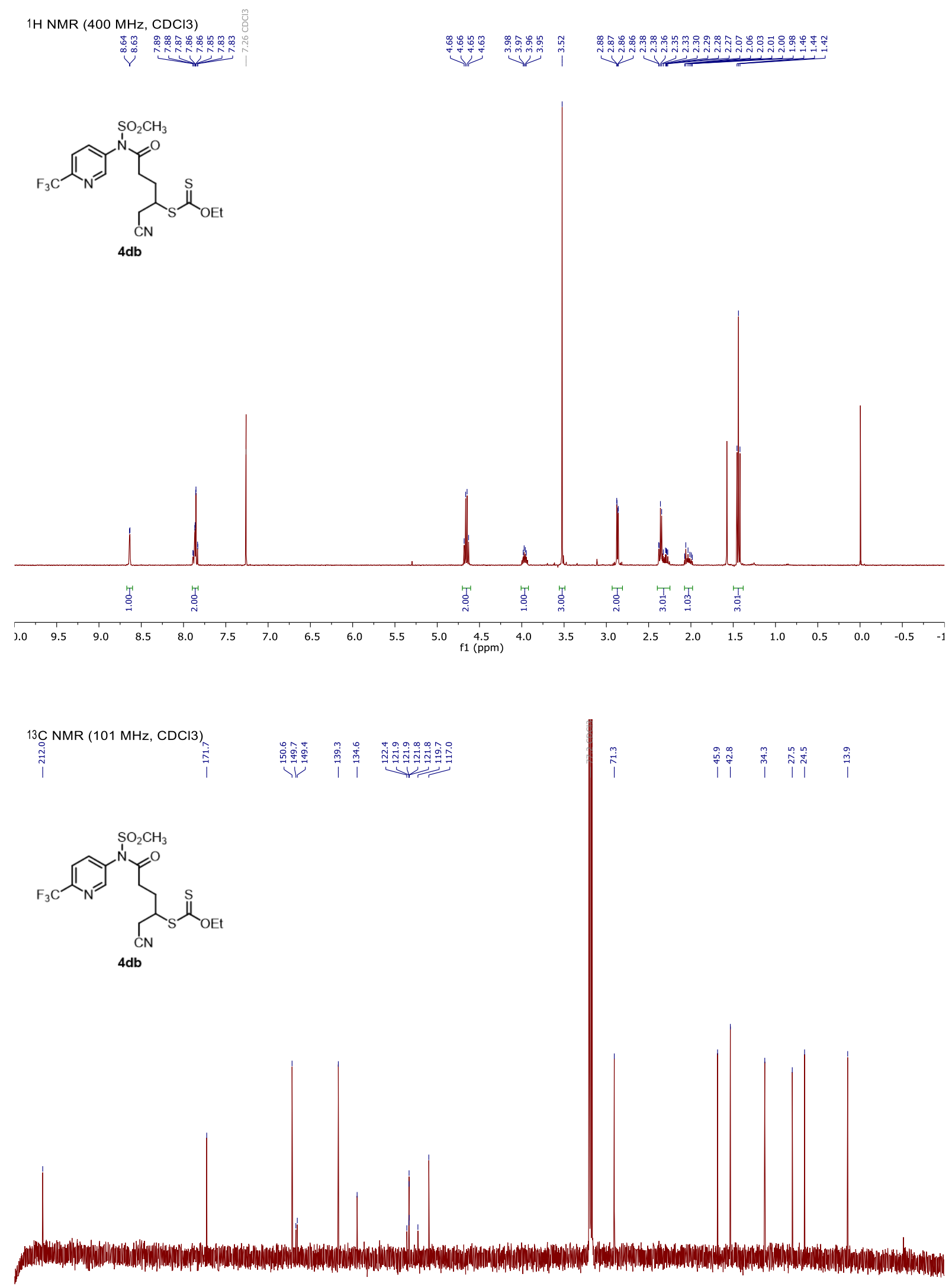

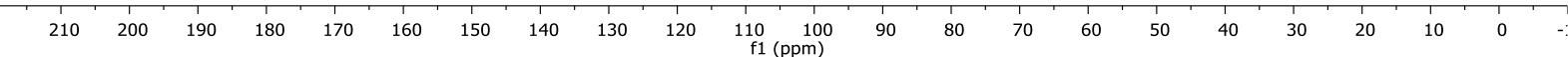




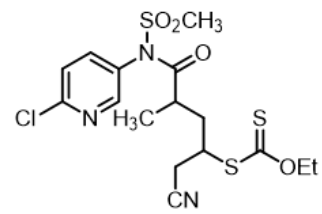

$4 \mathrm{eb}$

הับ

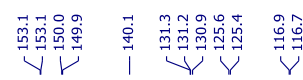

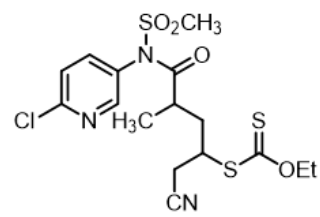

4eb

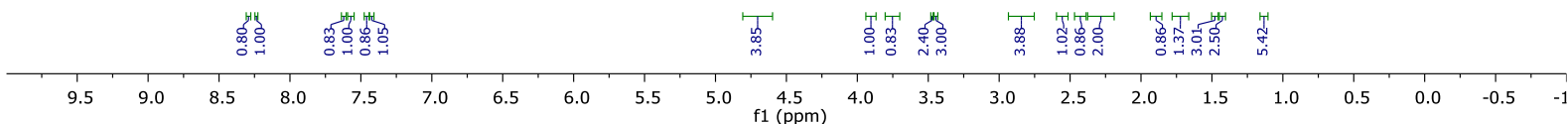

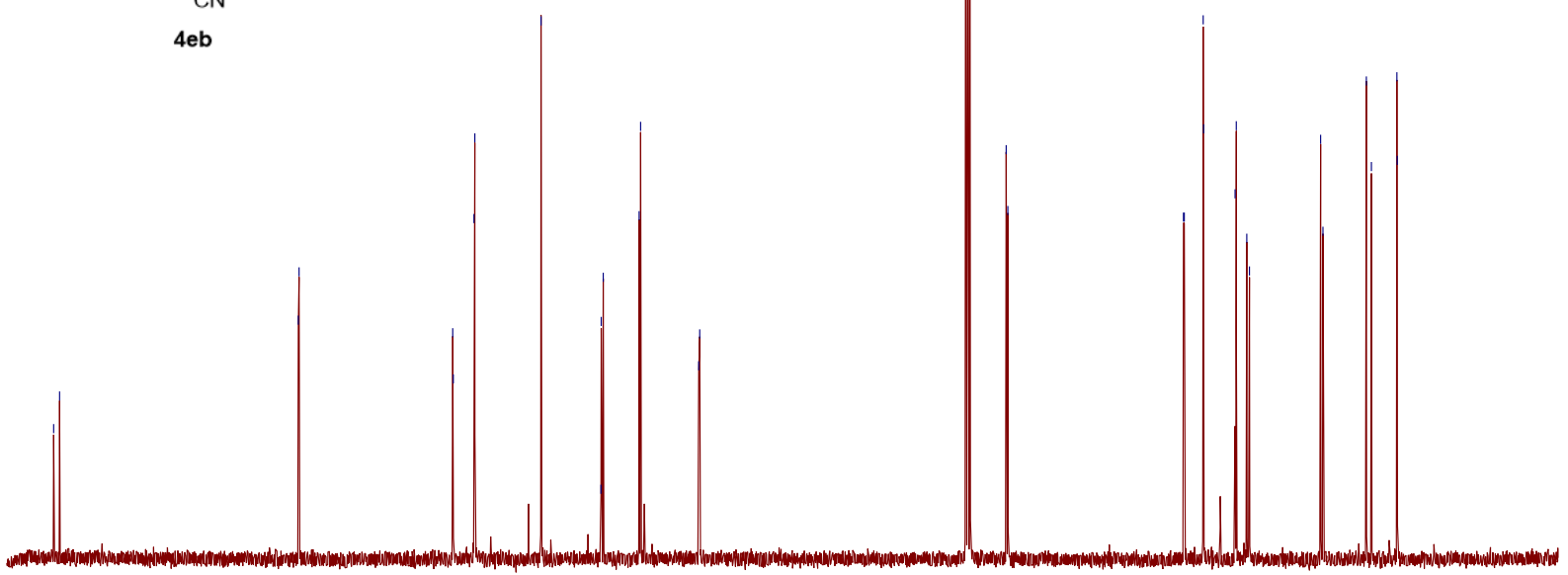

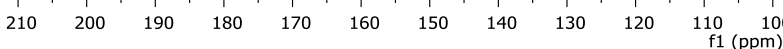



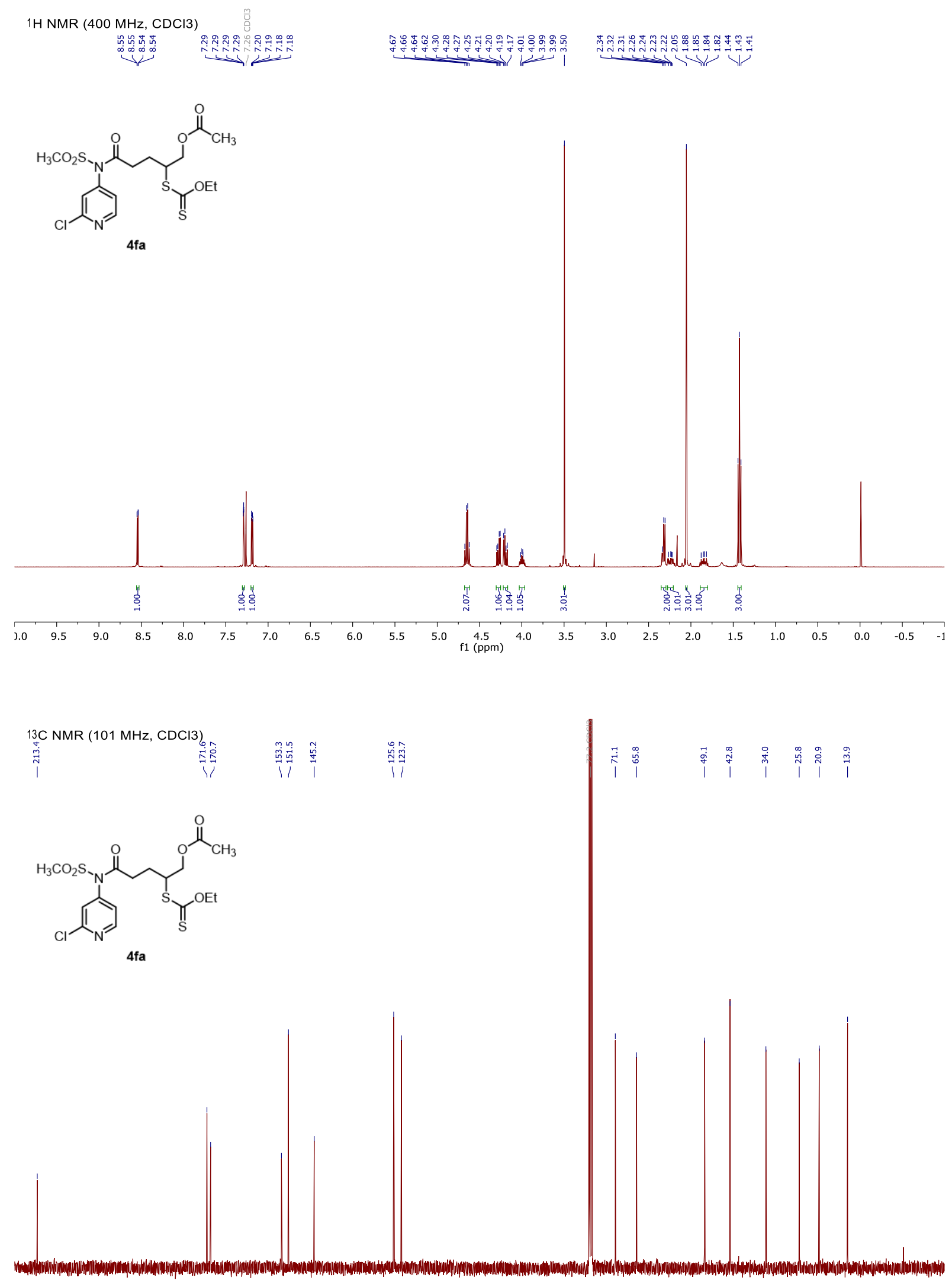

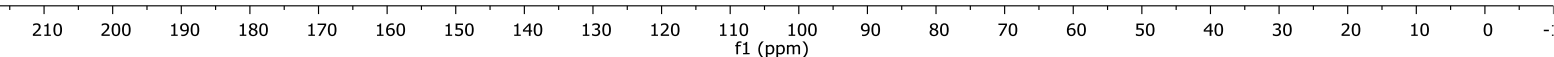



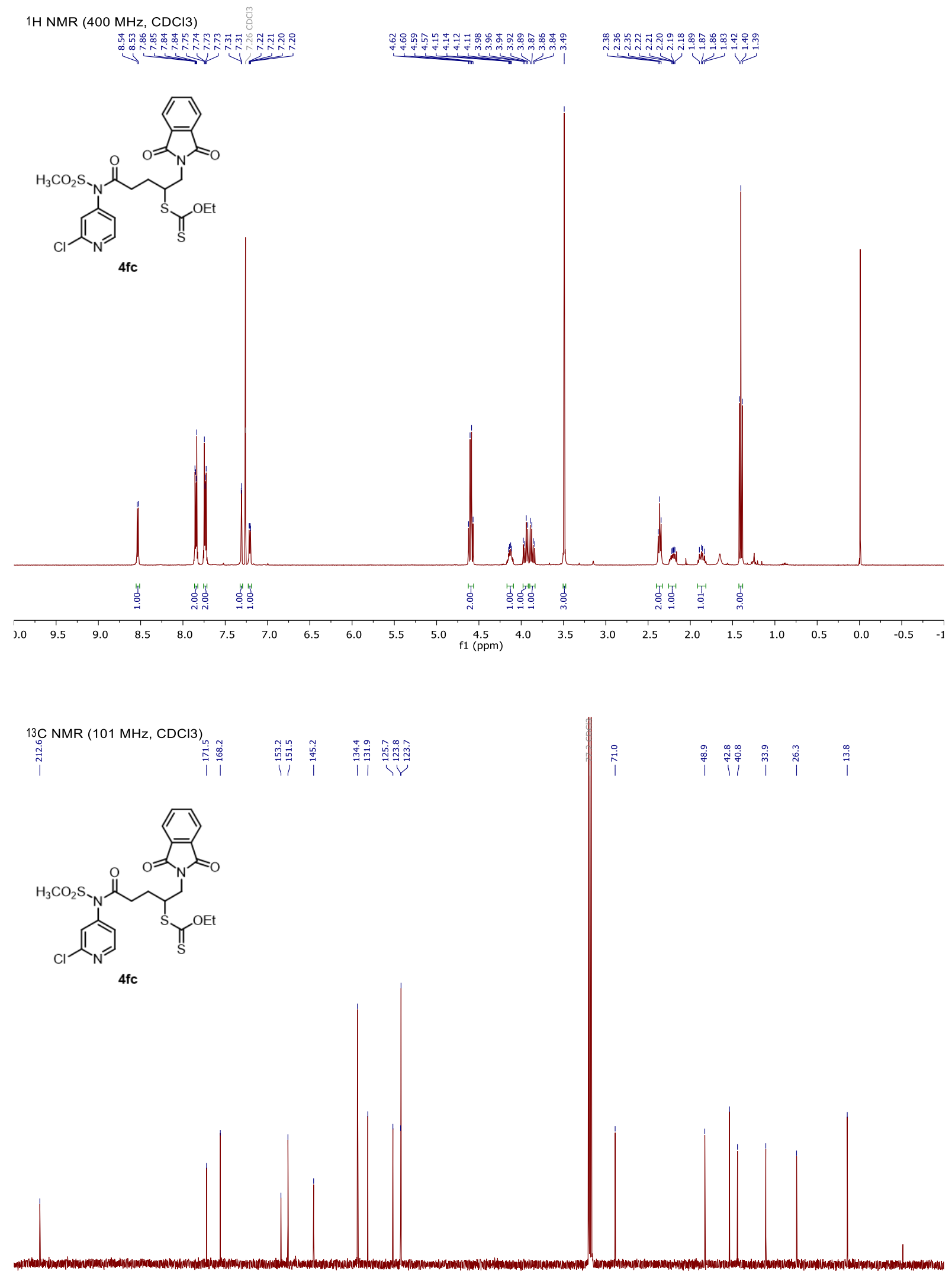

$\begin{array}{llllllllllllllllllllllllllllllll}210 & 200 & 190 & 180 & 170 & 160 & 150 & 140 & 130 & 120 & 110 & 100 & 90 & 80 & 70 & 60 & 50 & 40 & 30 & 20 & 10 & 0 & -\end{array}$ 

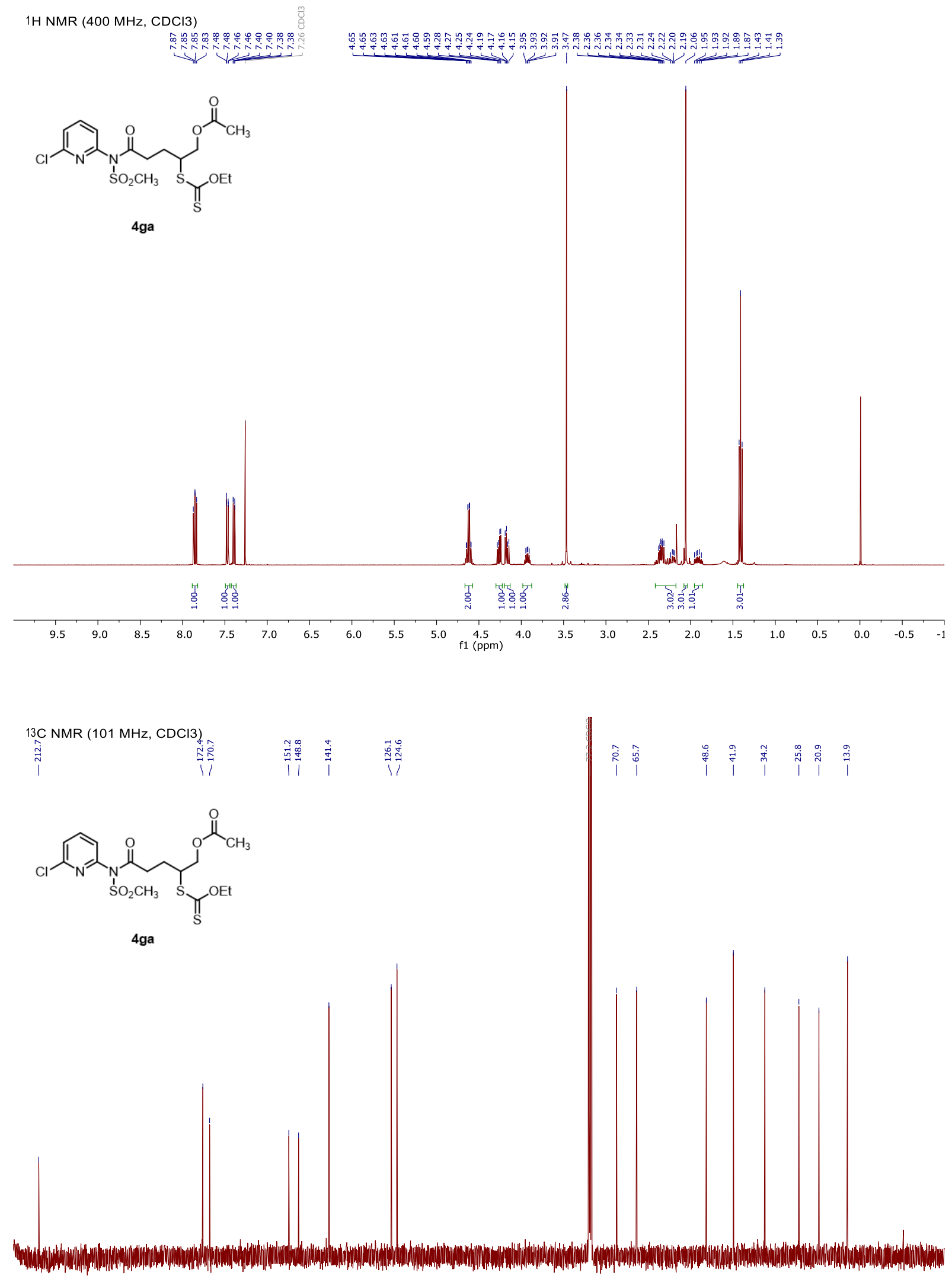

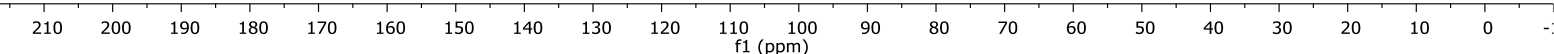



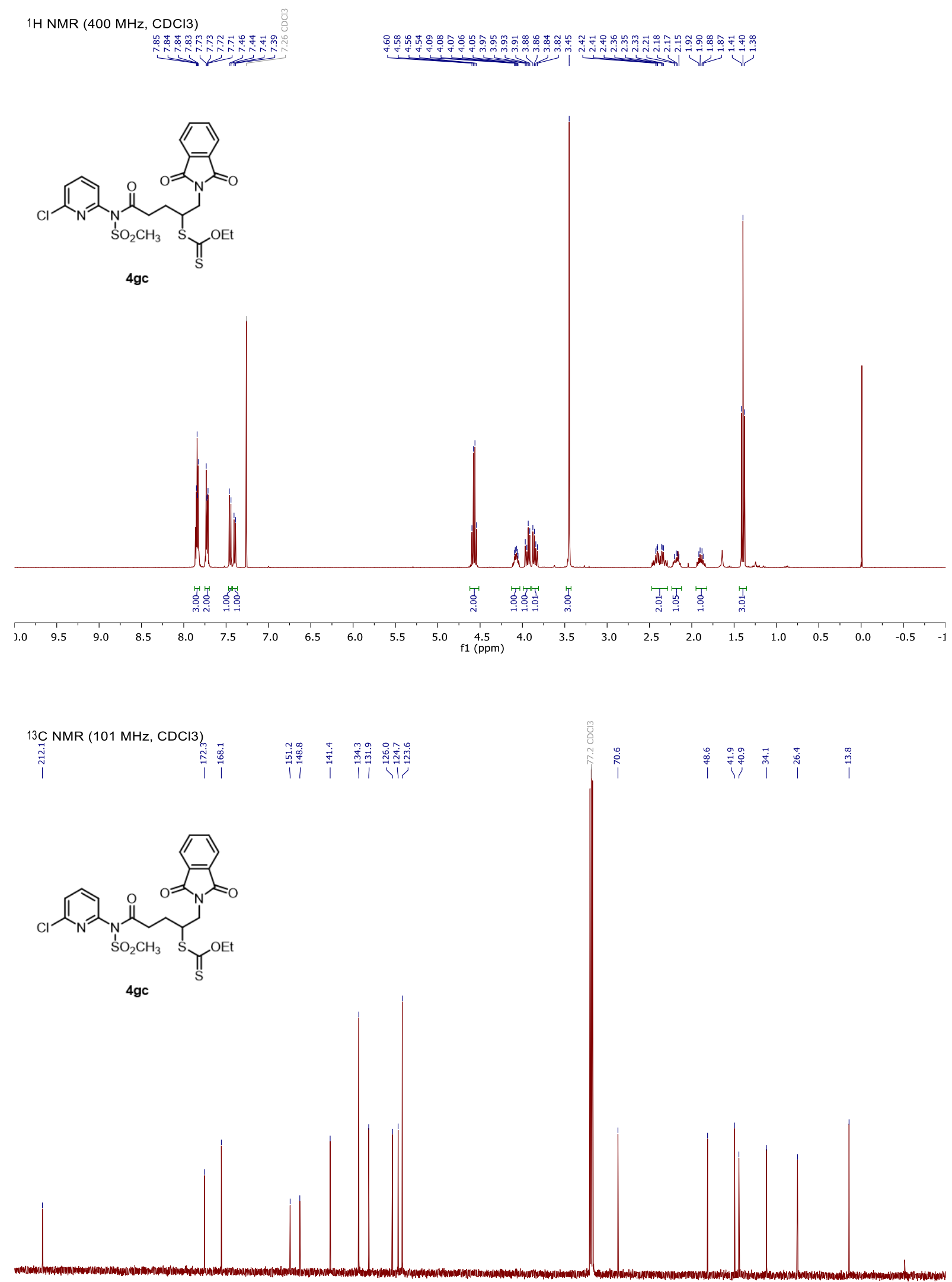

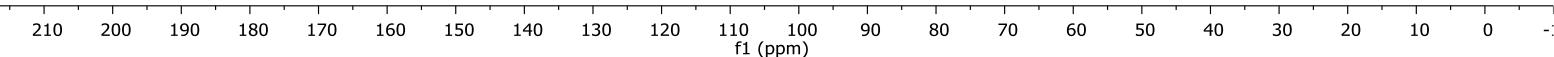




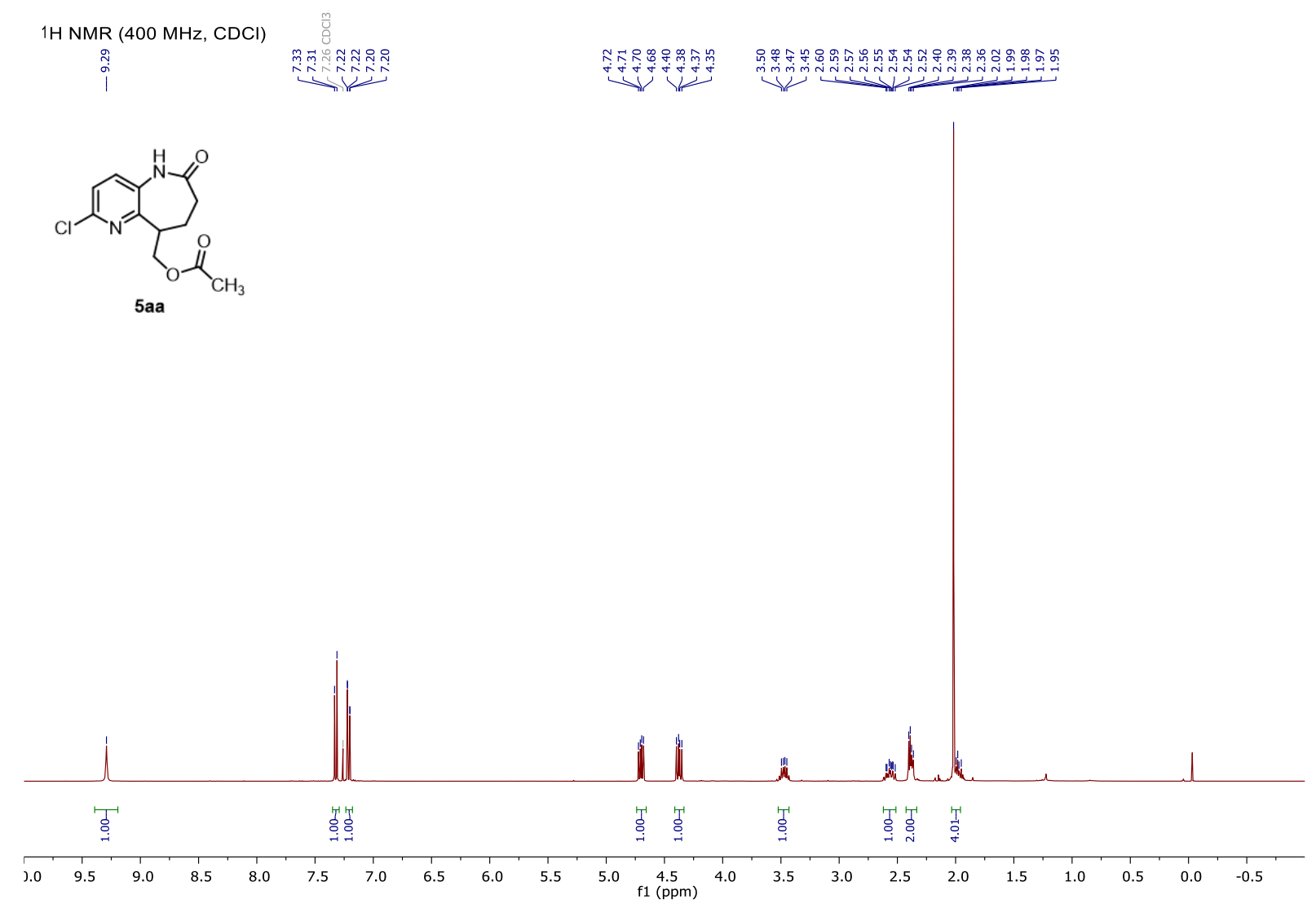

13C NMR (101 MHz, CDCl)

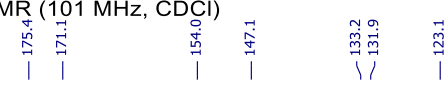

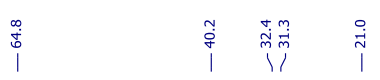
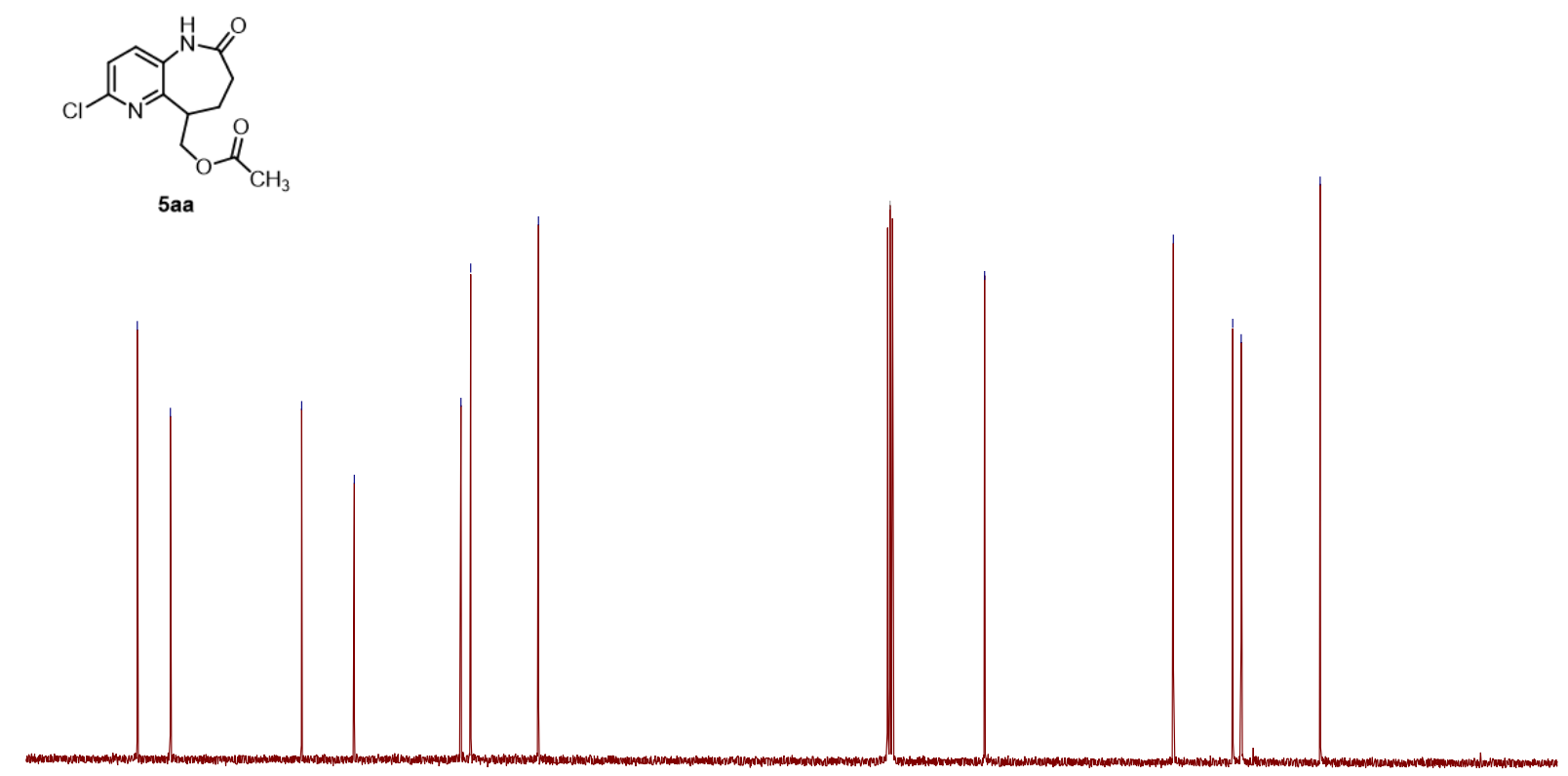

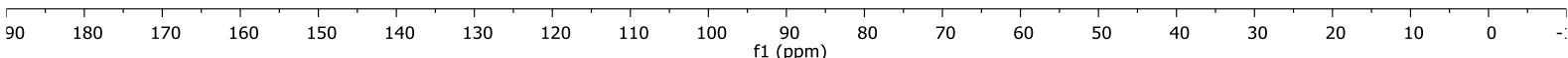


1H NMR (400 MHz, DMSO)

cond<smiles>N#CCC1CCC(=O)Nc2ccc(Cl)nc21</smiles>

$5 \mathrm{ab}$

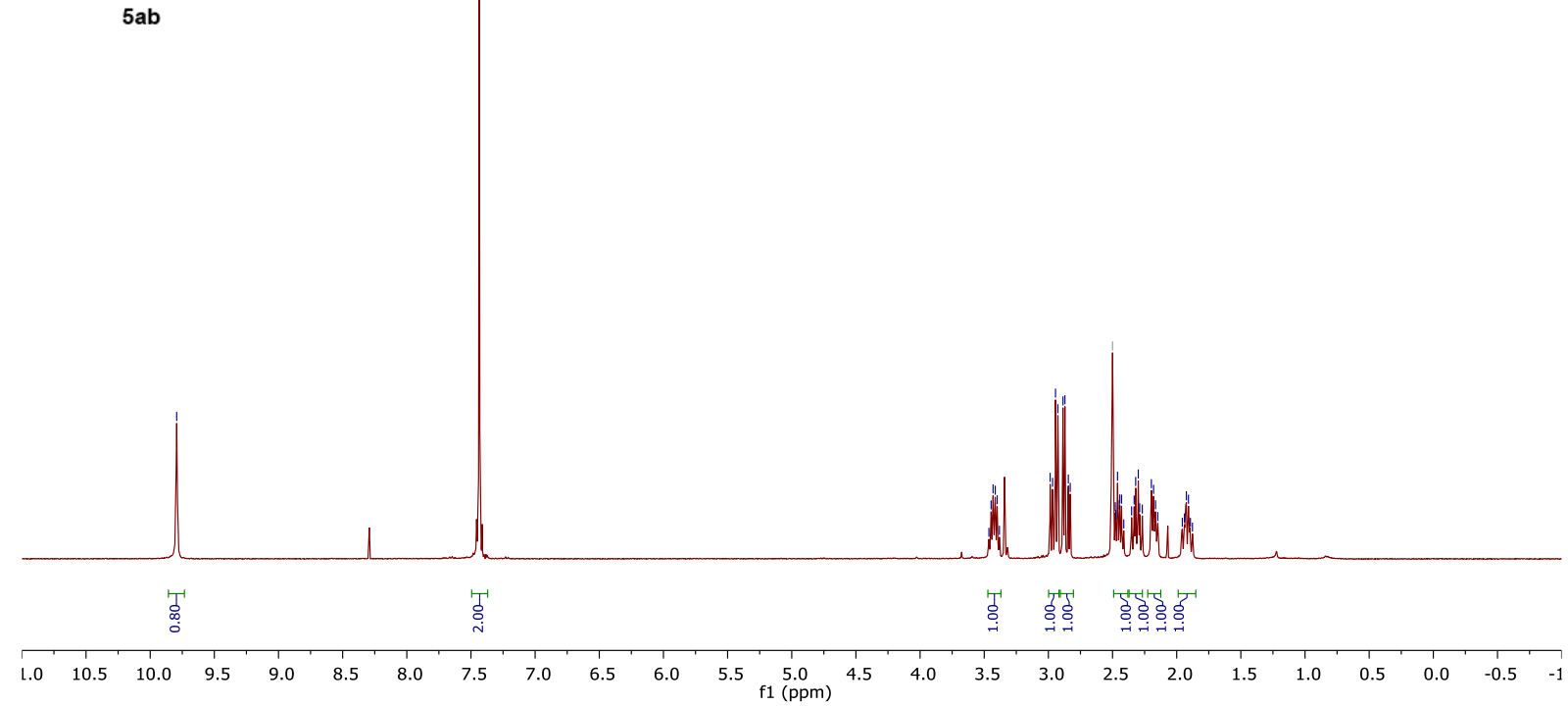

13C NMR (101 MHz, DMSO)

点<smiles>N#CCC1CCC(=O)Nc2ccc(Cl)nc21</smiles>

5ab
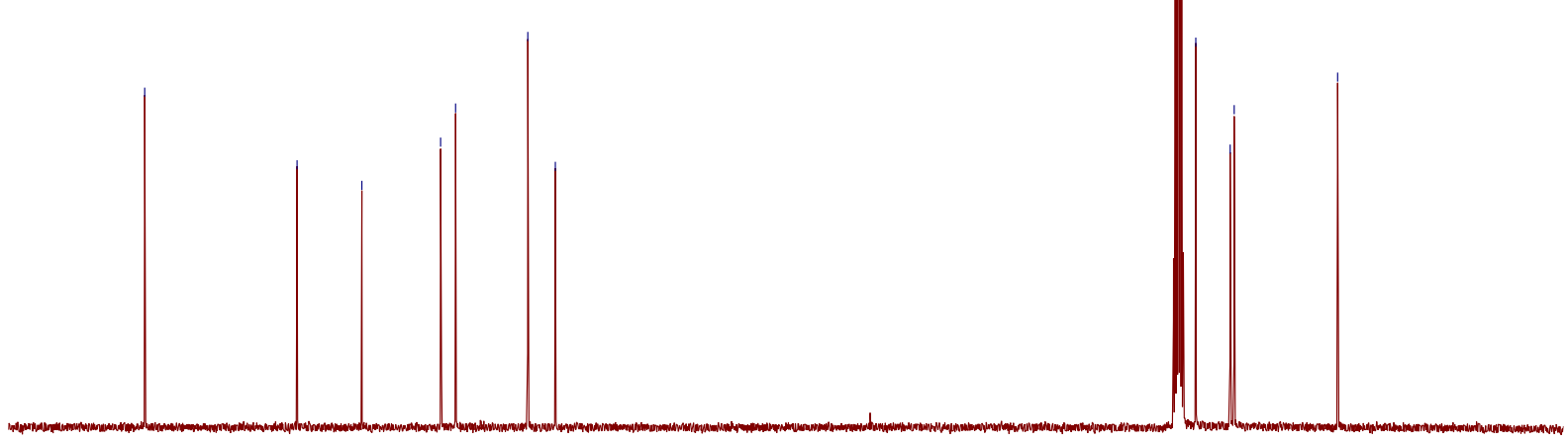
1H NMR (400 MHz, DMSO)

ֻ̊.

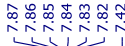<smiles>O=C1CCC(CN2C(=O)c3ccccc3C2=O)c2nc(Cl)ccc2N1</smiles>

$5 a c$

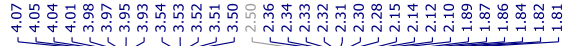

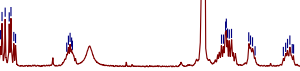

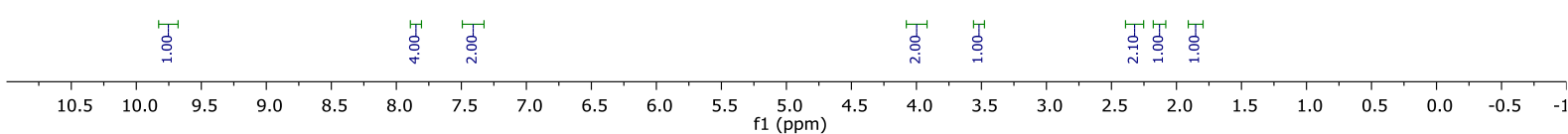

13C NMR (101 MHz, DMSO)

।<smiles>O=C1CCC(CN2C(=O)c3ccccc3C2=O)c2nc(Cl)ccc2N1</smiles>

5 ac

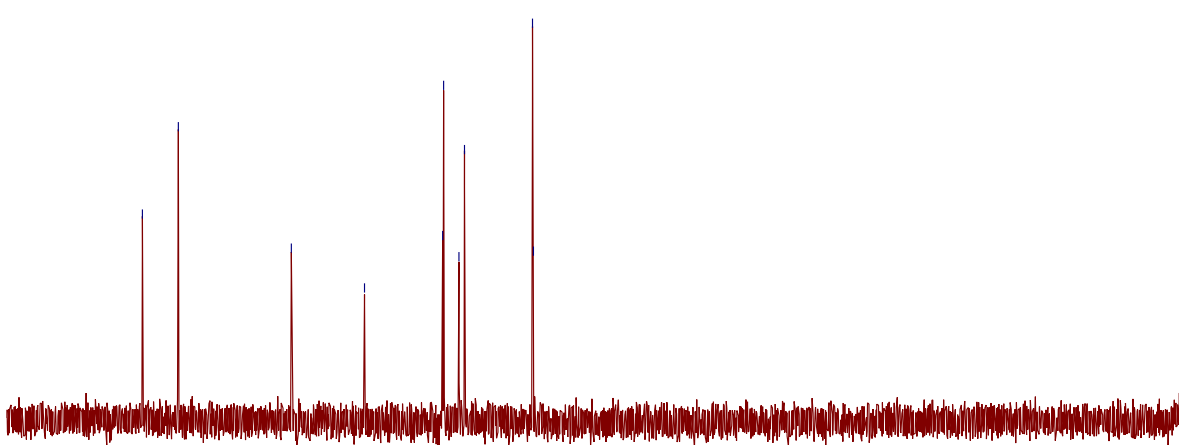

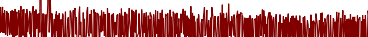

\begin{tabular}{lllllllllllllllllllllllll}
\hline 90 & 180 & 170 & 160 & 150 & 140 & 130 & 120 & 110 & 100 & 90 & 80 & 70 & 60 & 50 & 40 & 30 & 20 & 10 & 0 & -
\end{tabular} 

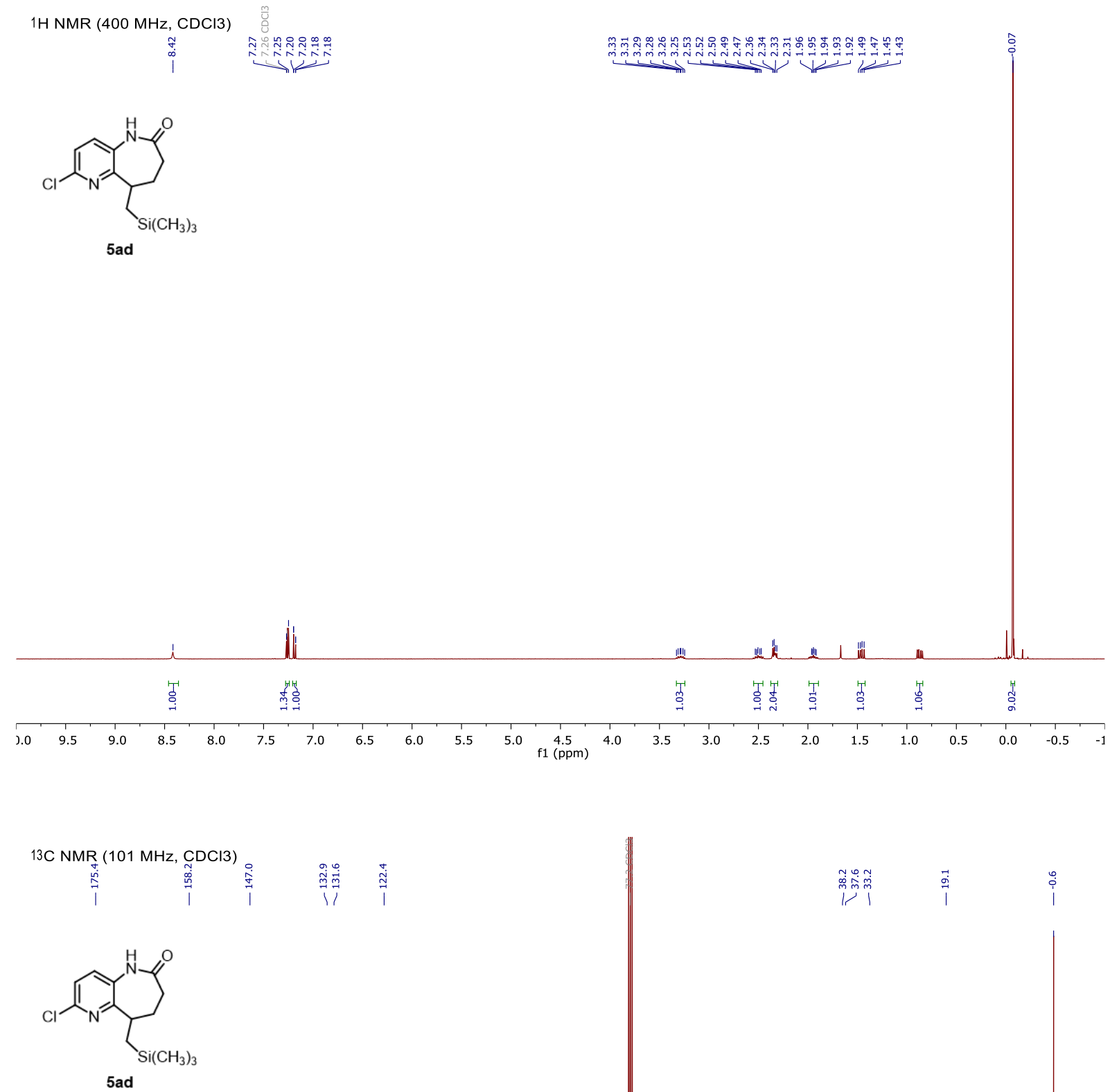

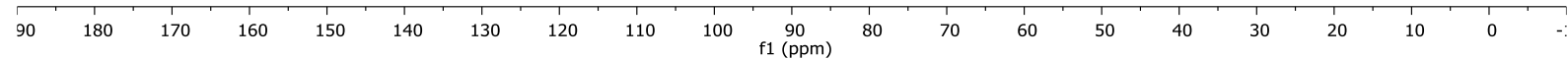


1H NMR (400 MHz, DMSO)

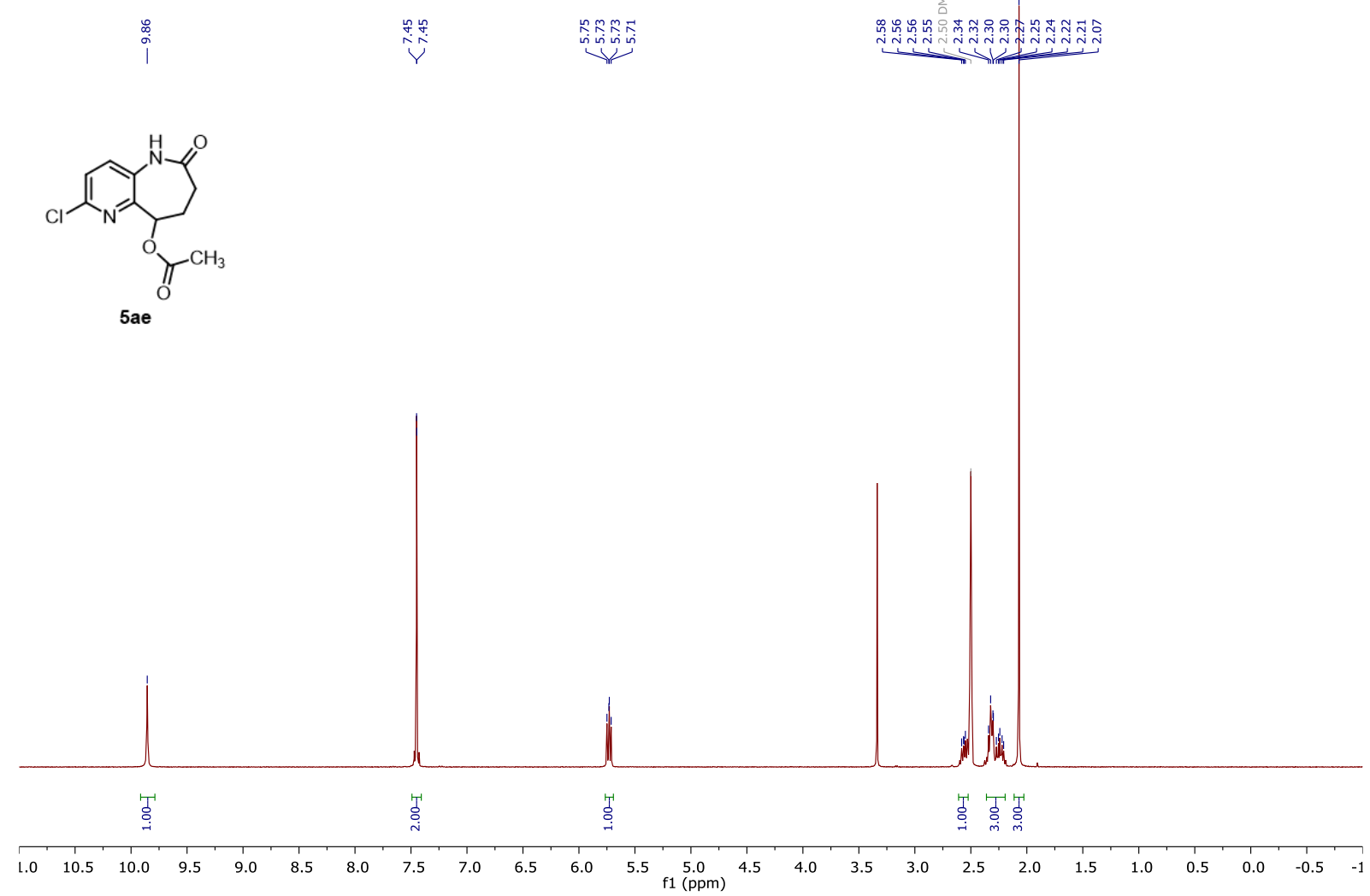

13C NMR (101 MHz, DMSO)

尗焦

$\stackrel{1}{i}$<smiles>CC(=O)OC1CCC(=O)Nc2ccc(Cl)nc21</smiles>

5ae

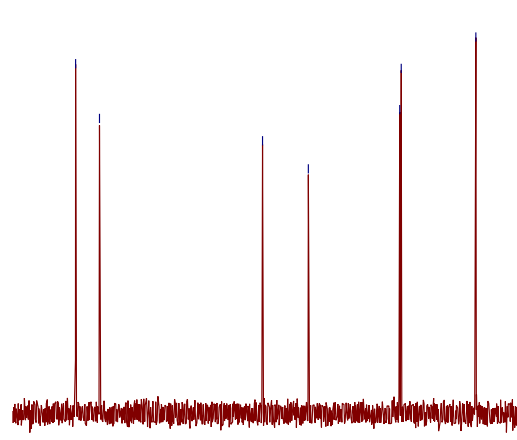

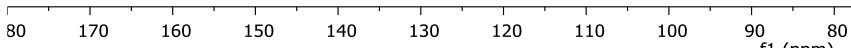




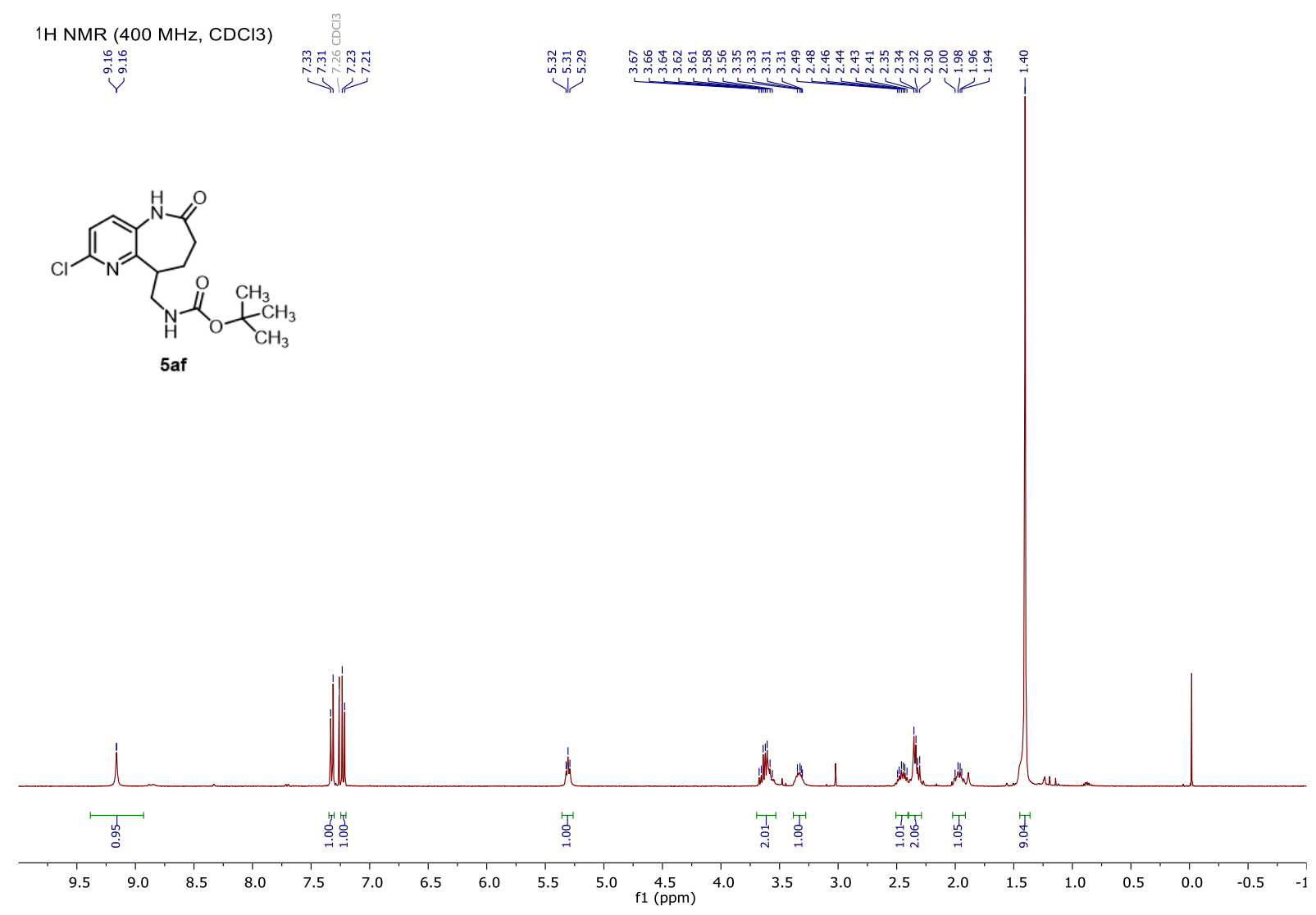

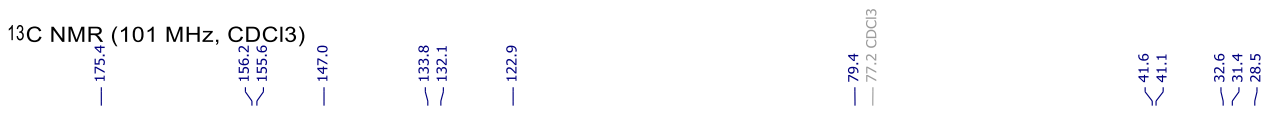

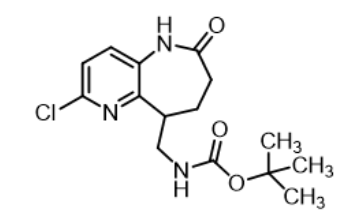

5af

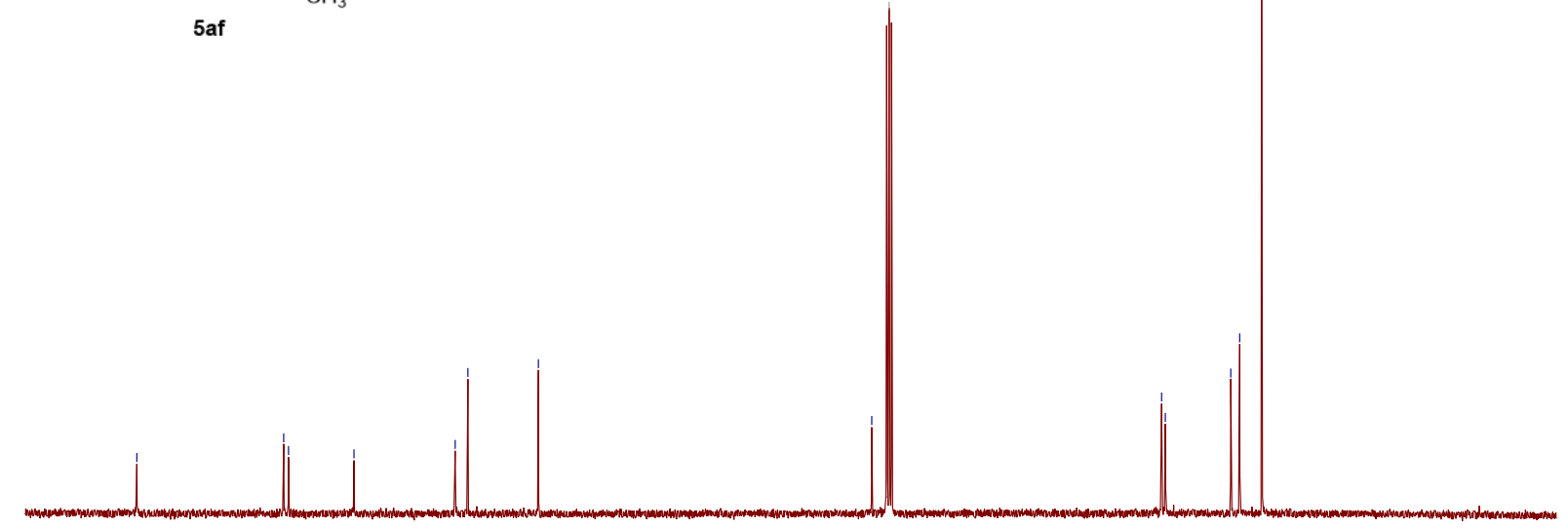

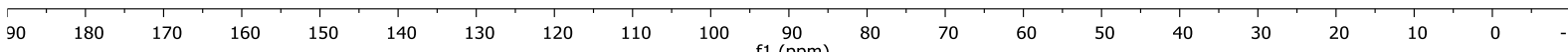



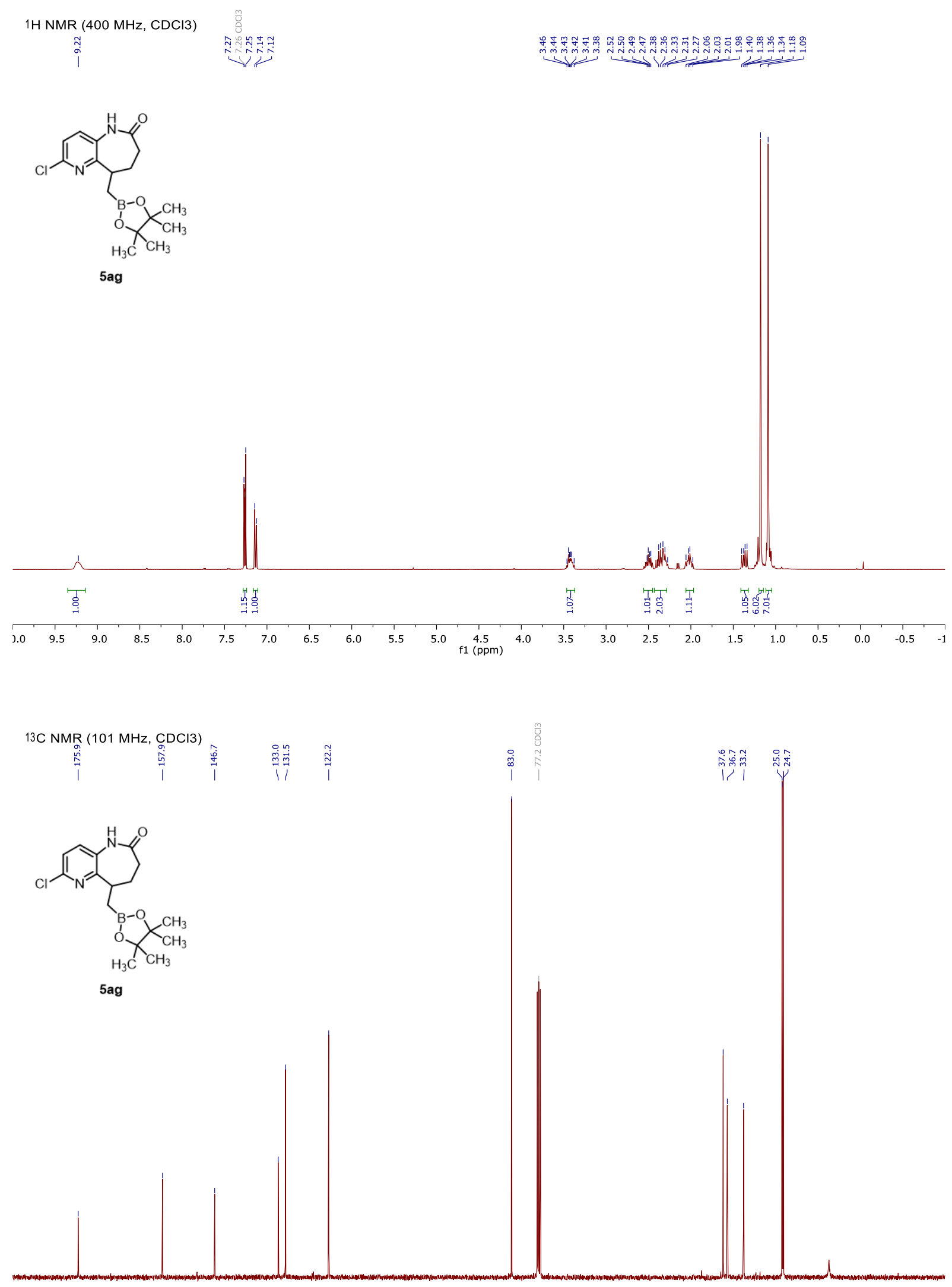

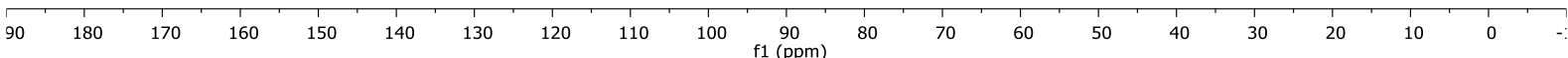



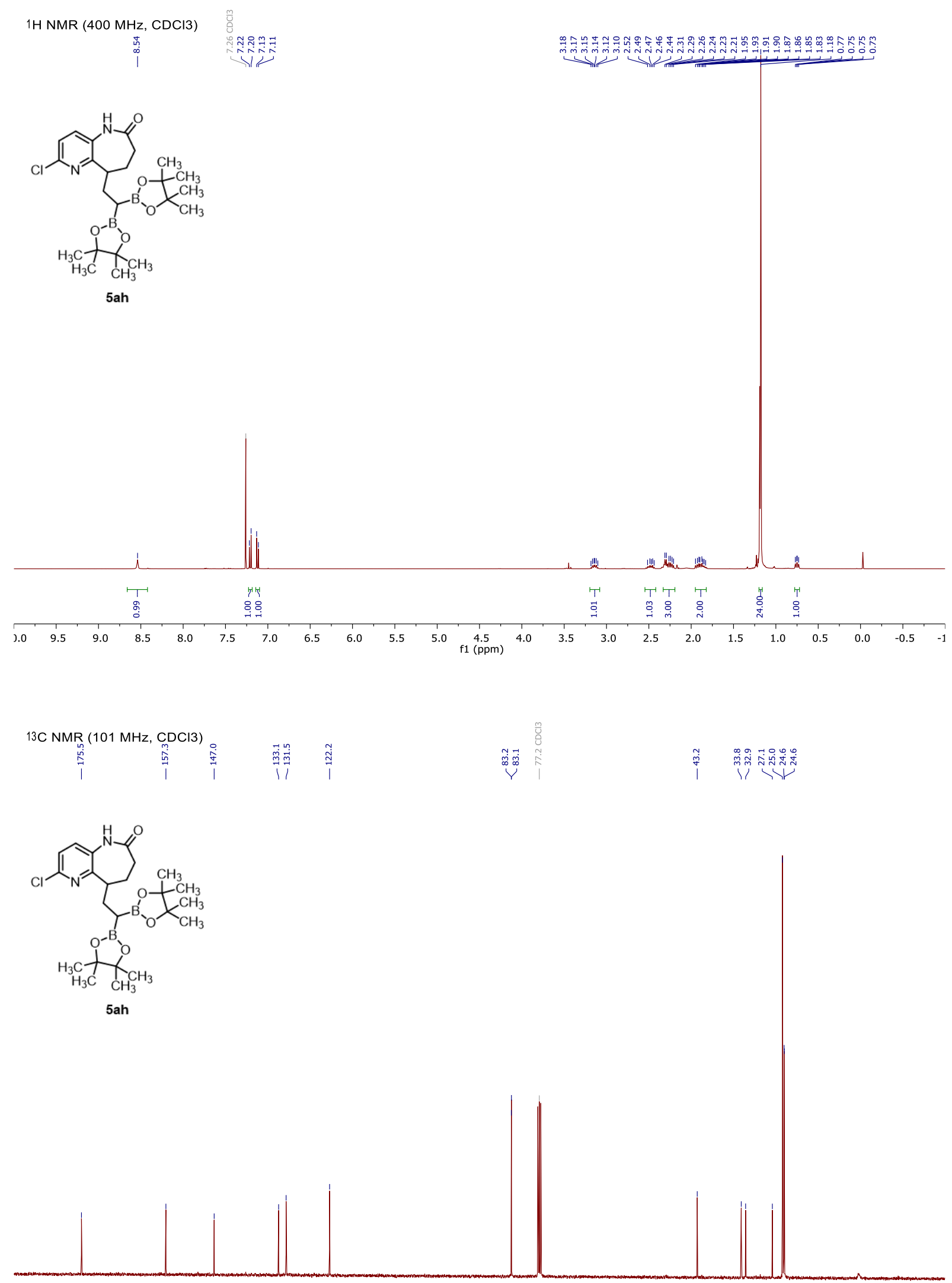

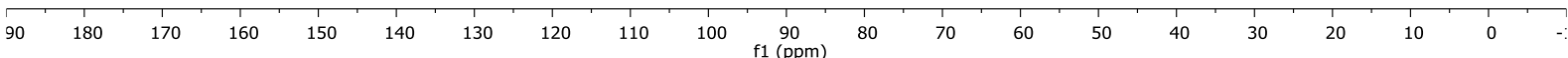


$1 \mathrm{H} \mathrm{NMR}(400 \mathrm{MHz}$, Acetone)

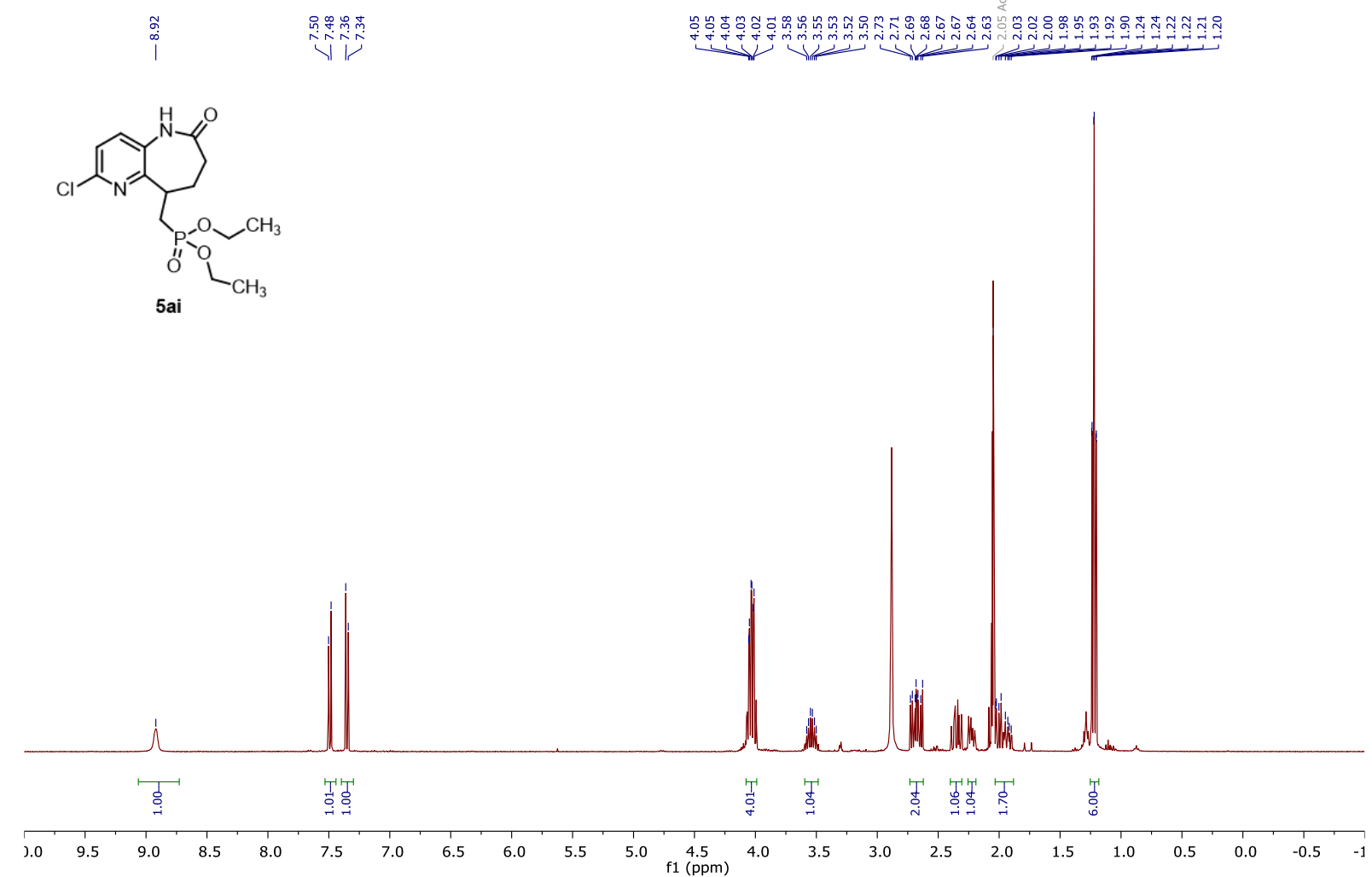

13C NMR (101 MHz, Acetone)
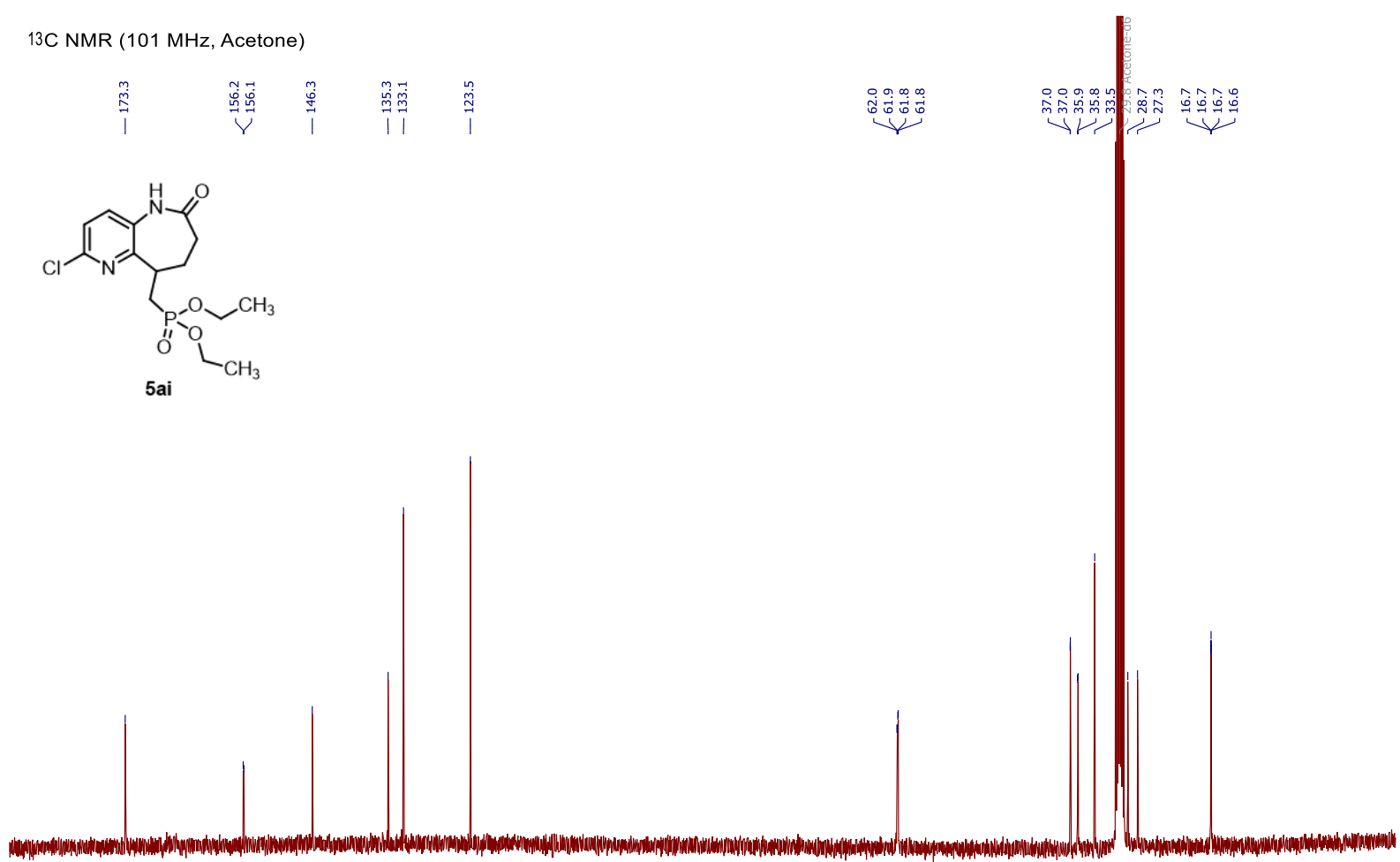

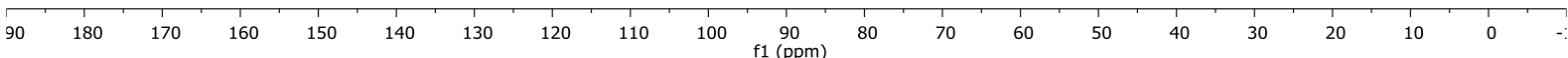



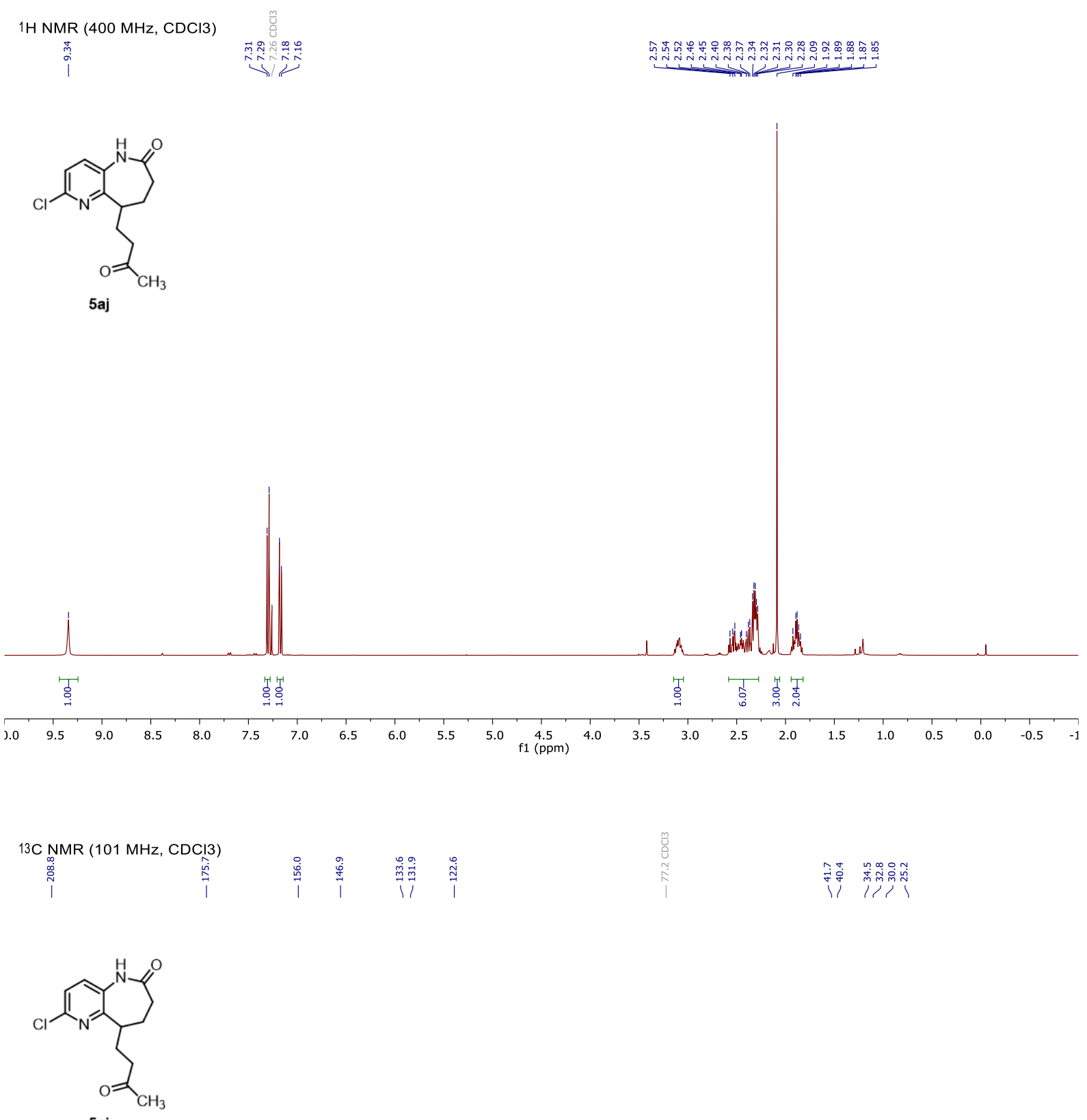

5aj

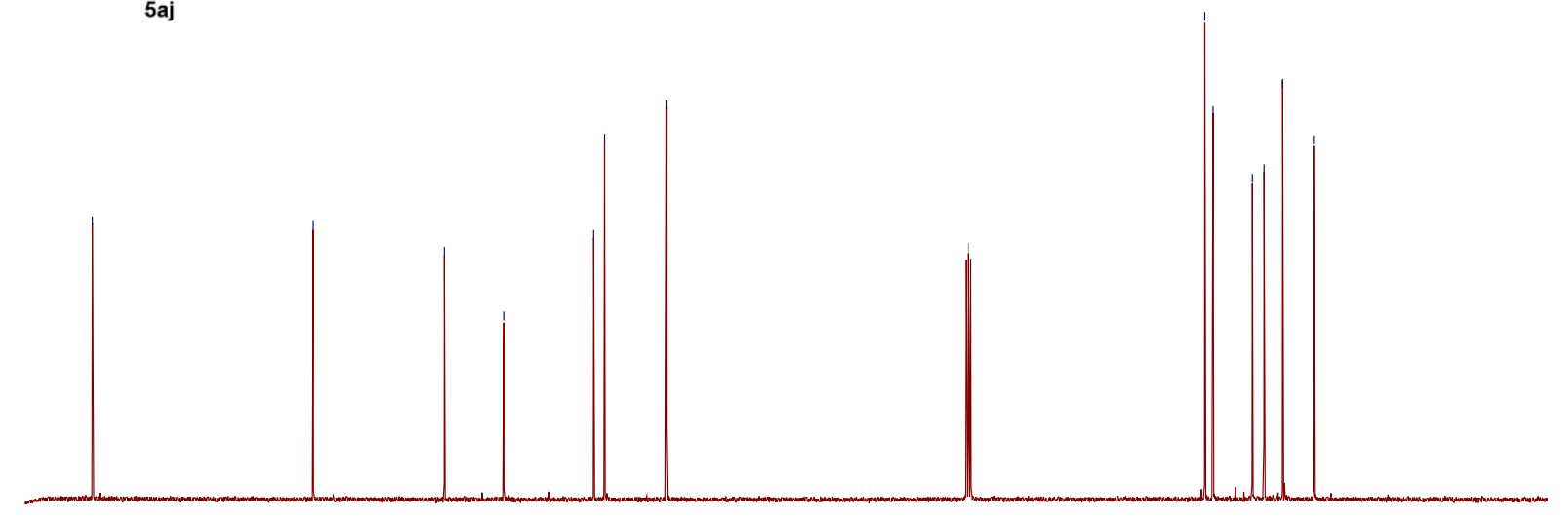

$\begin{array}{lllllllllllllllllllllllll}210 & 200 & 190 & 180 & 170 & 160 & 150 & 140 & 130 & 120 & 110 & 100 & 90 & 80 & 70 & 60 & 50 & 40 & 30 & 20 & 10 & 0 & -:\end{array}$ 


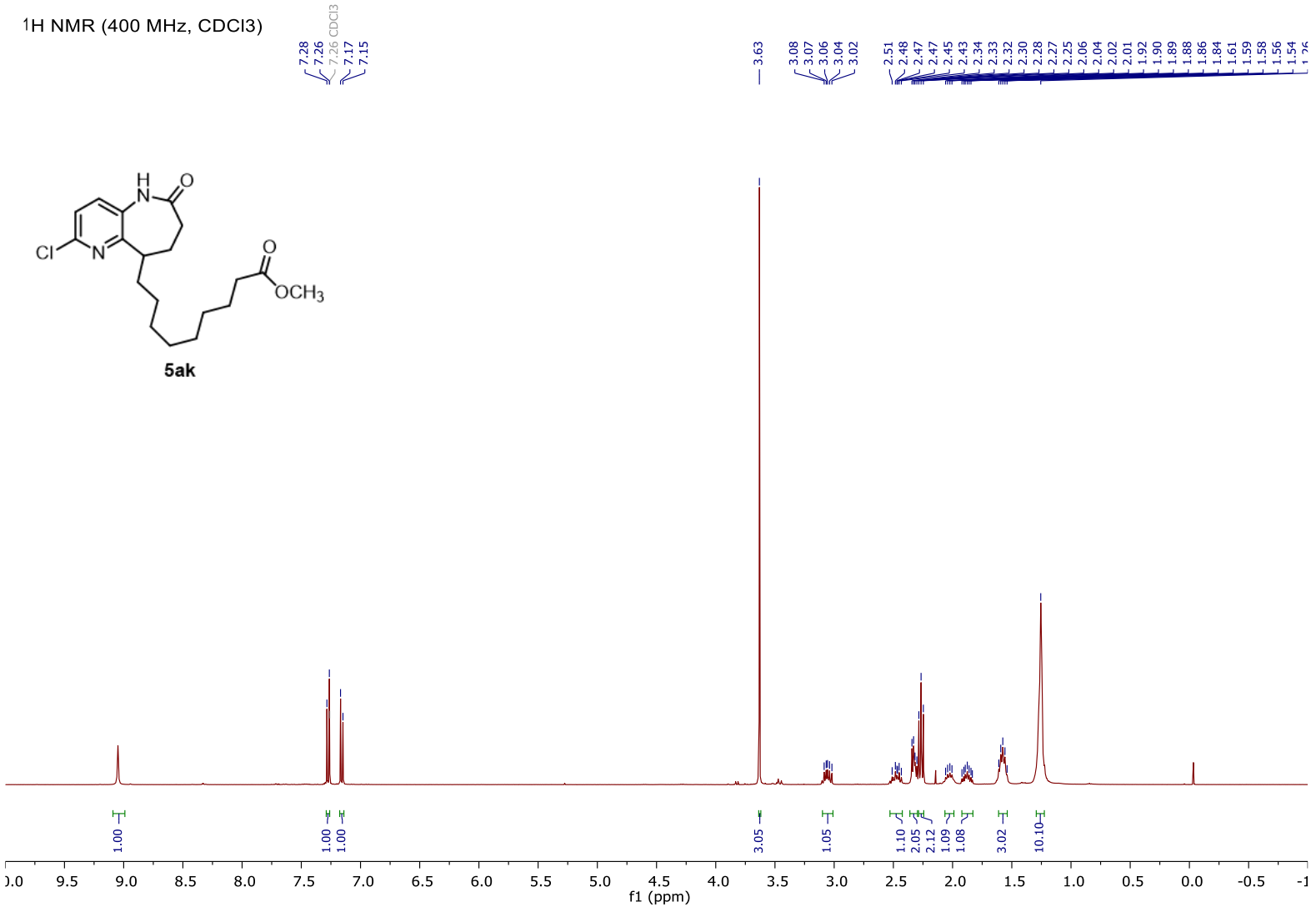

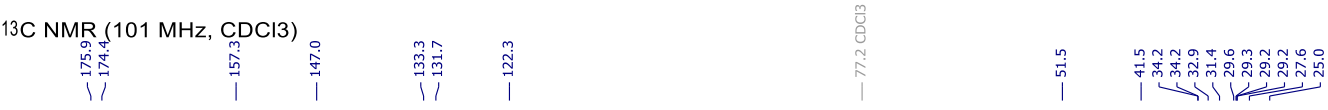
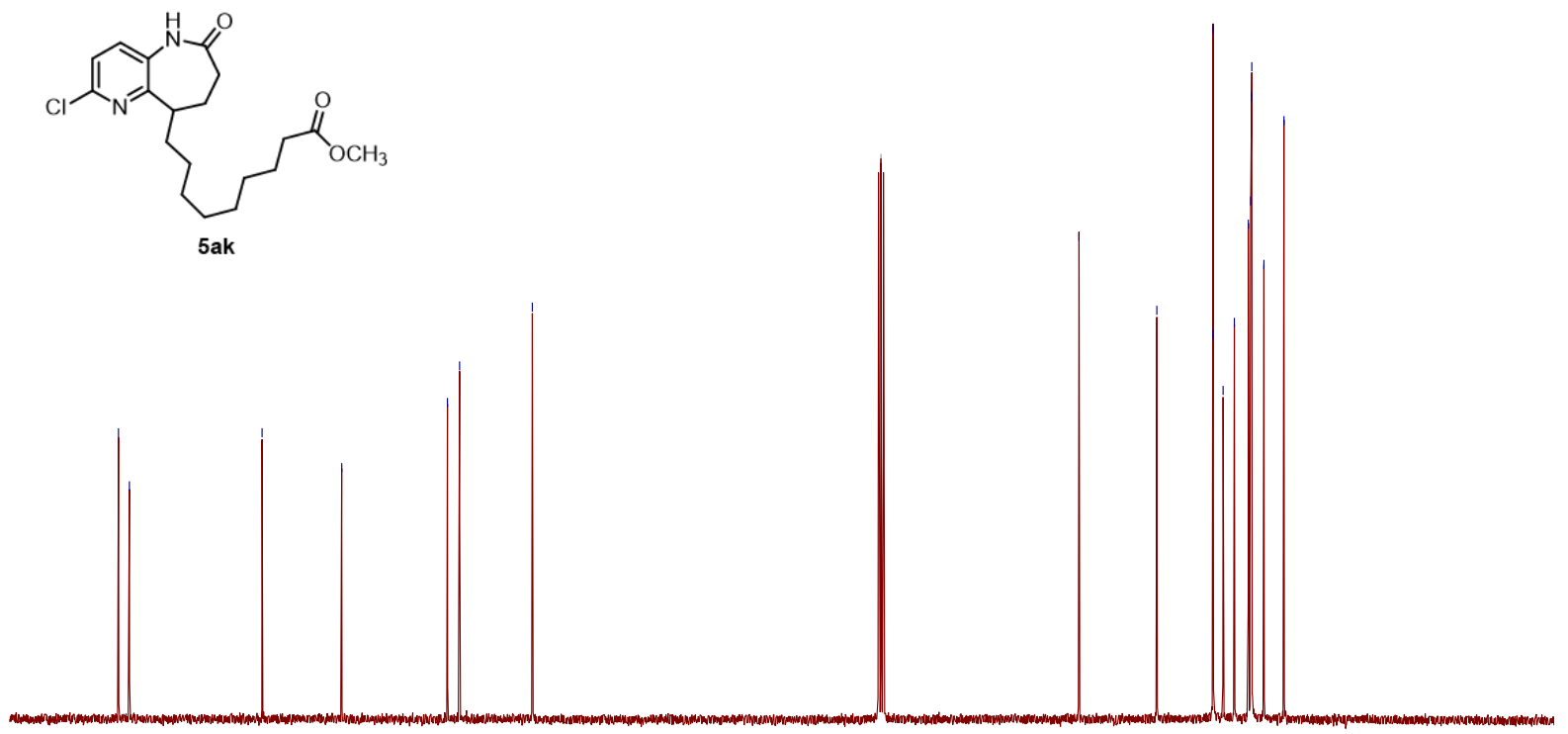

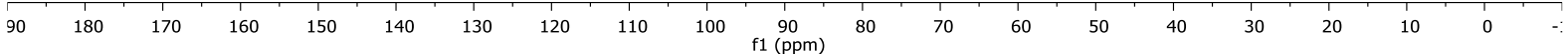



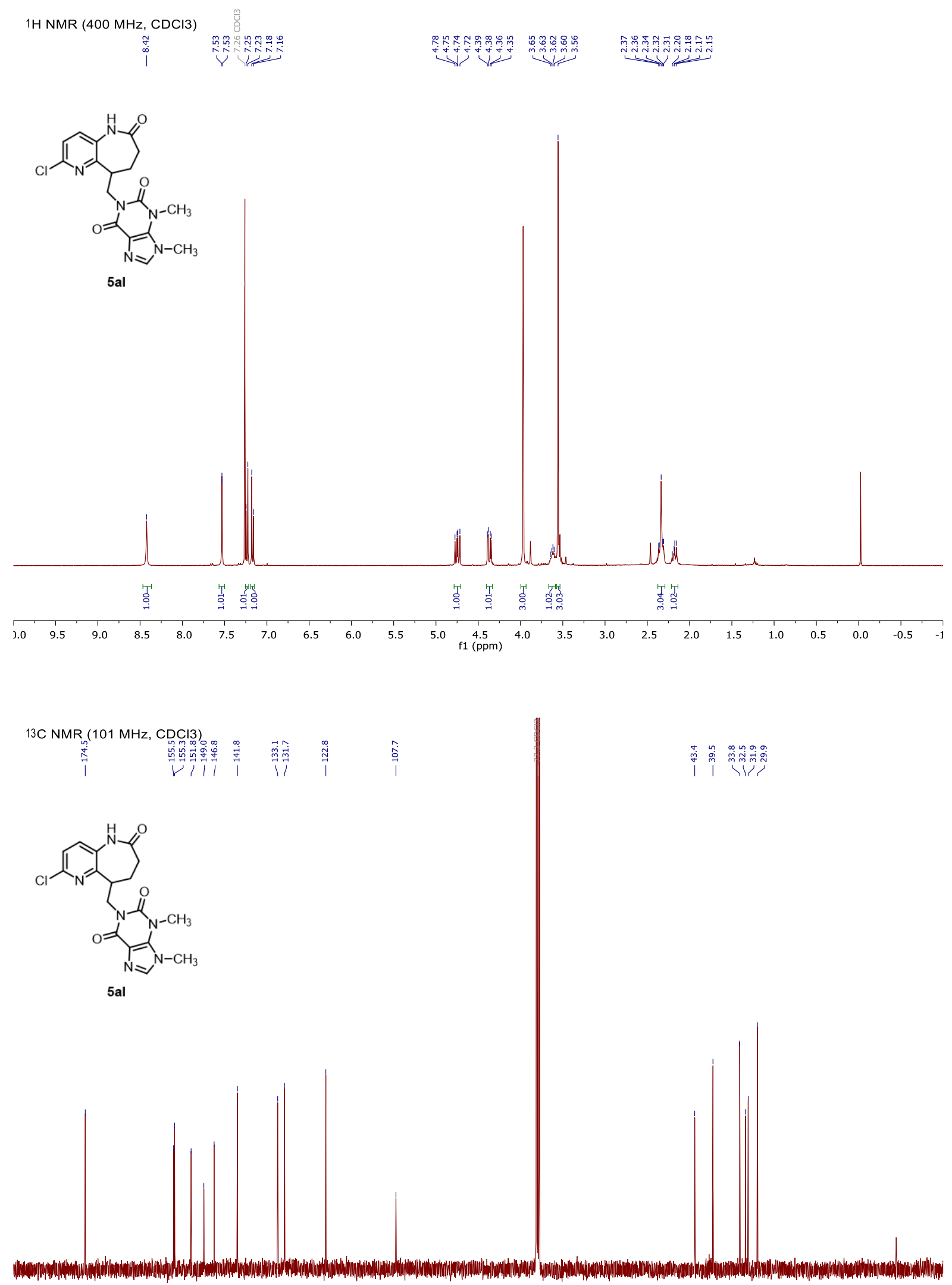

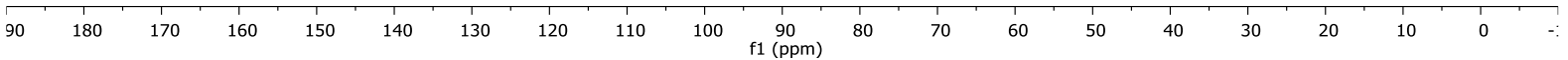



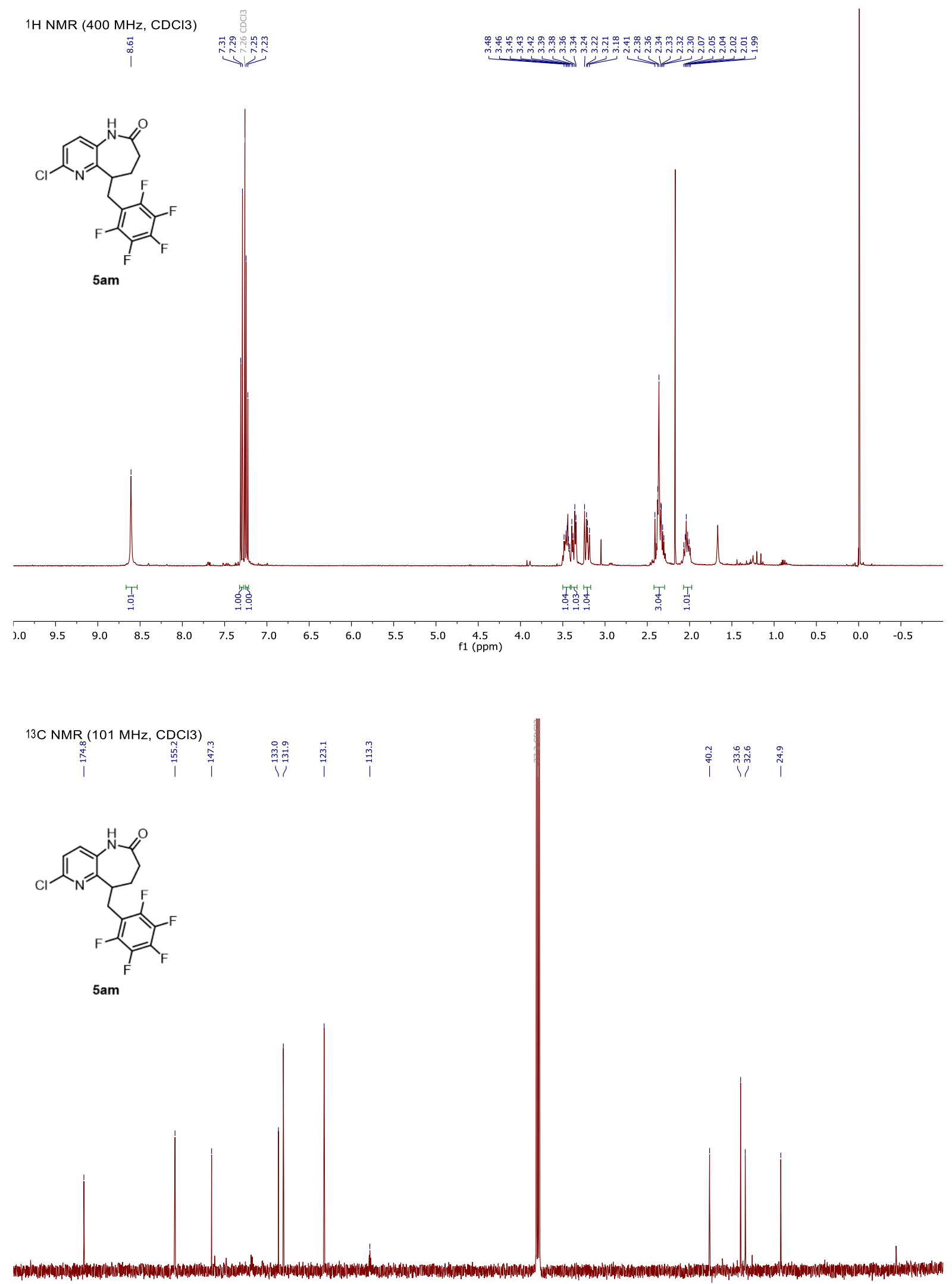

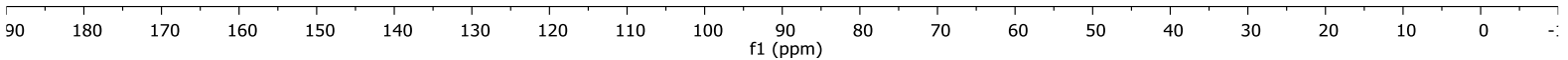



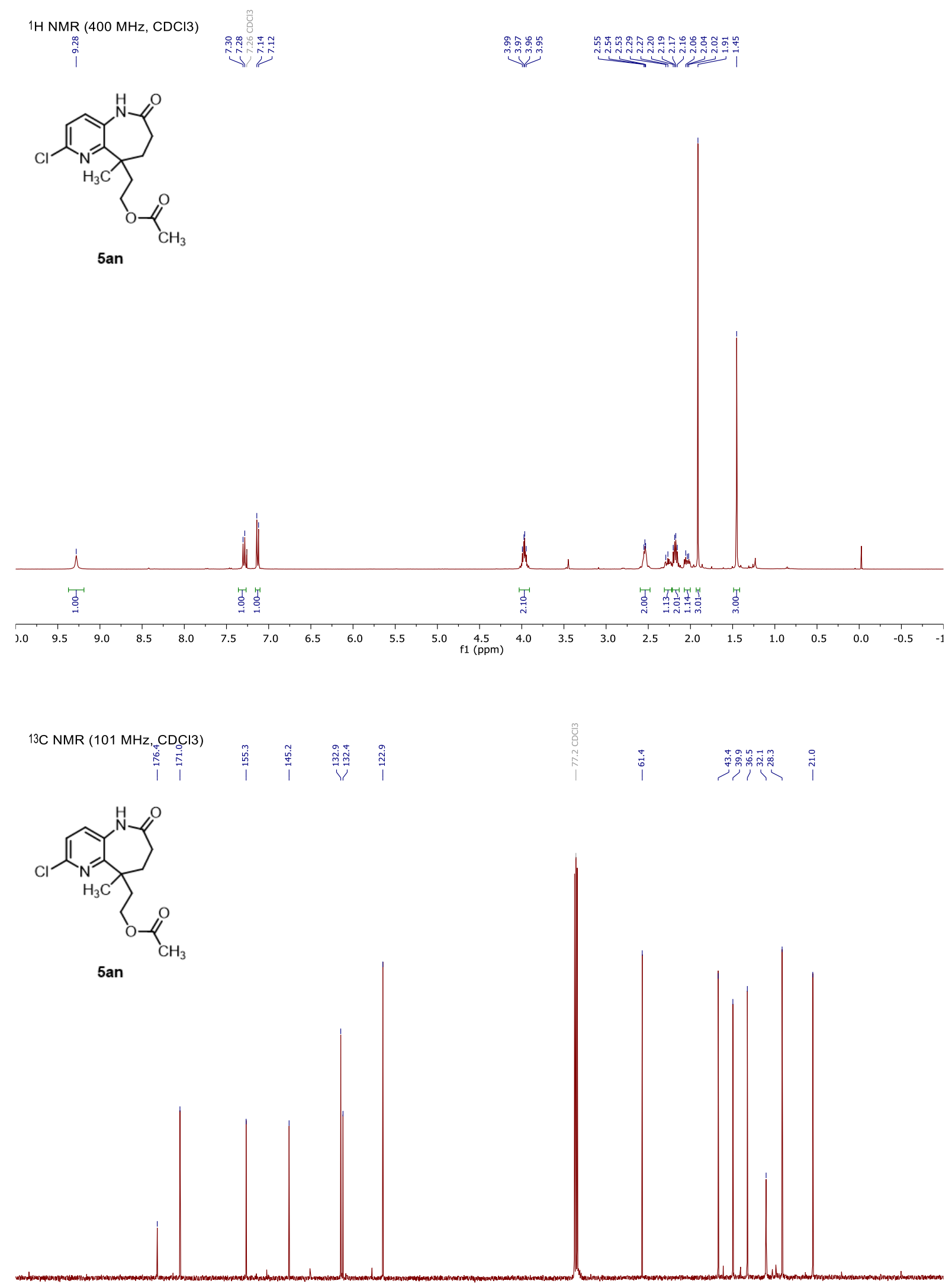

\begin{tabular}{lllllllllllllllllllllll}
\hline 10 & 200 & 190 & 180 & 170 & 160 & 150 & 140 & 130 & 120 & 110 & 100 & 10 & 80 & 70 & 60 & 50 & 40 & 30 & 20 & 10 & 0 & $-:$
\end{tabular} 

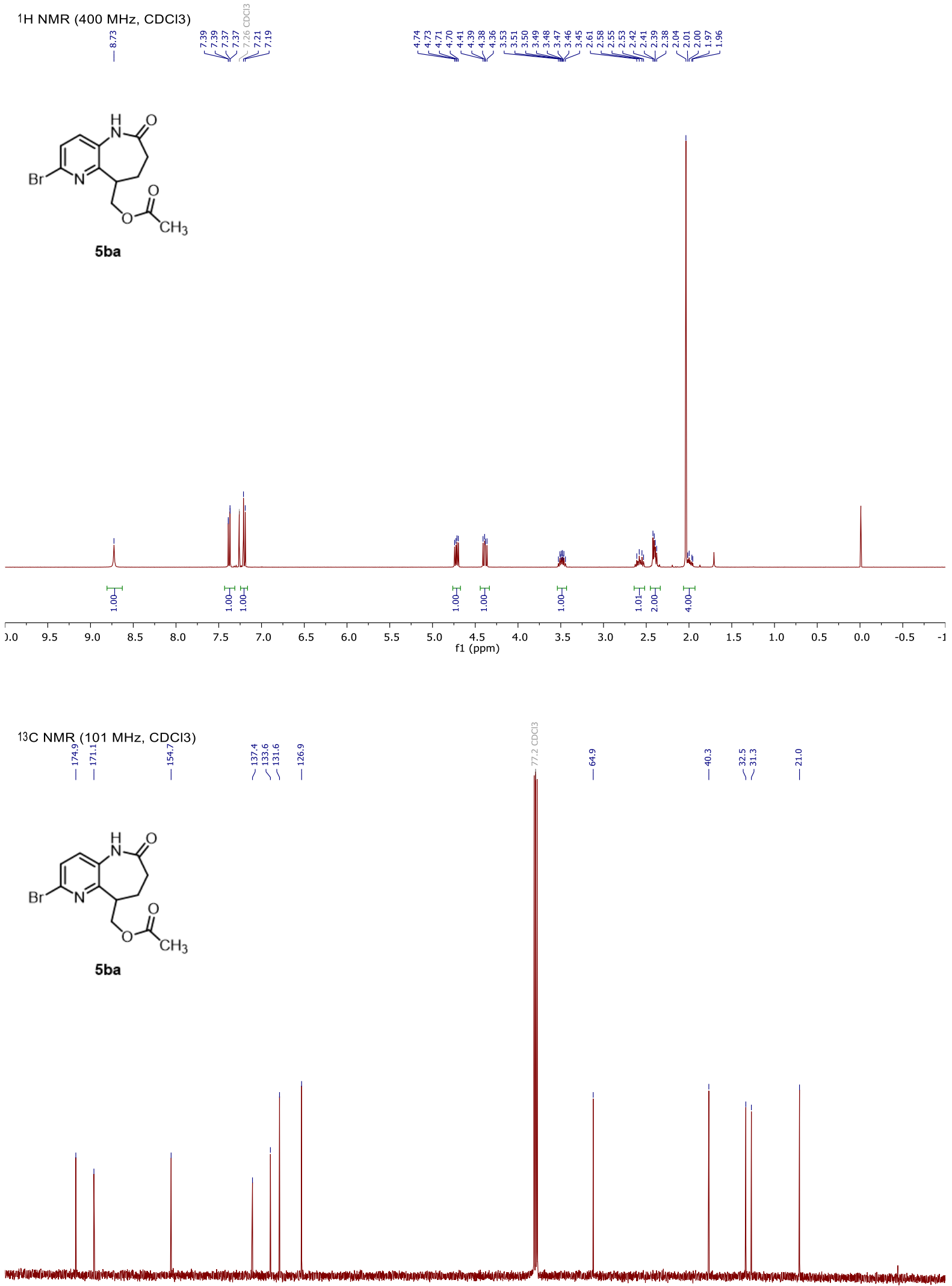

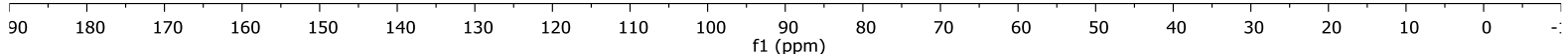



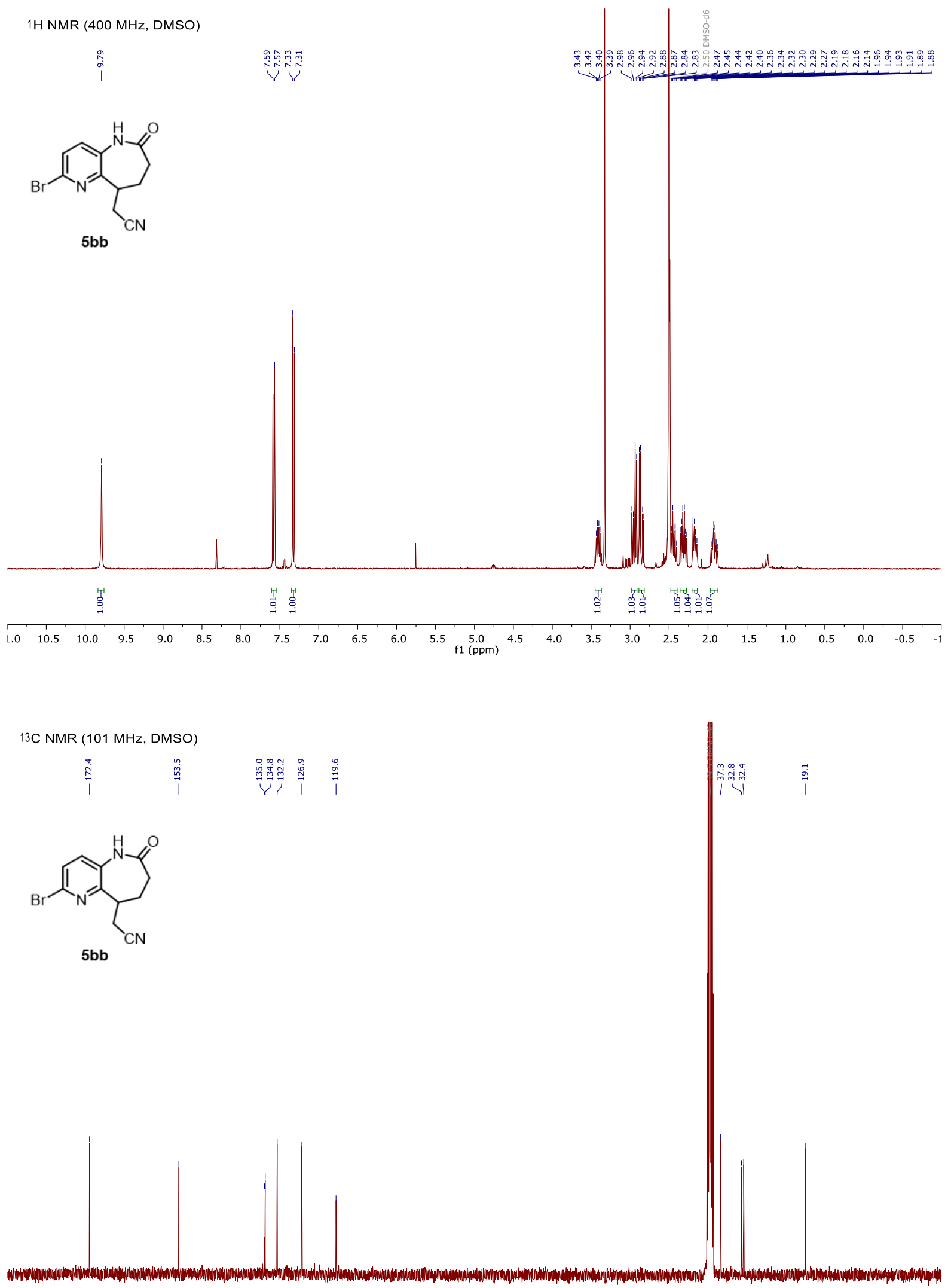

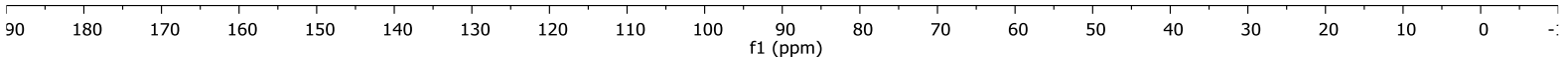




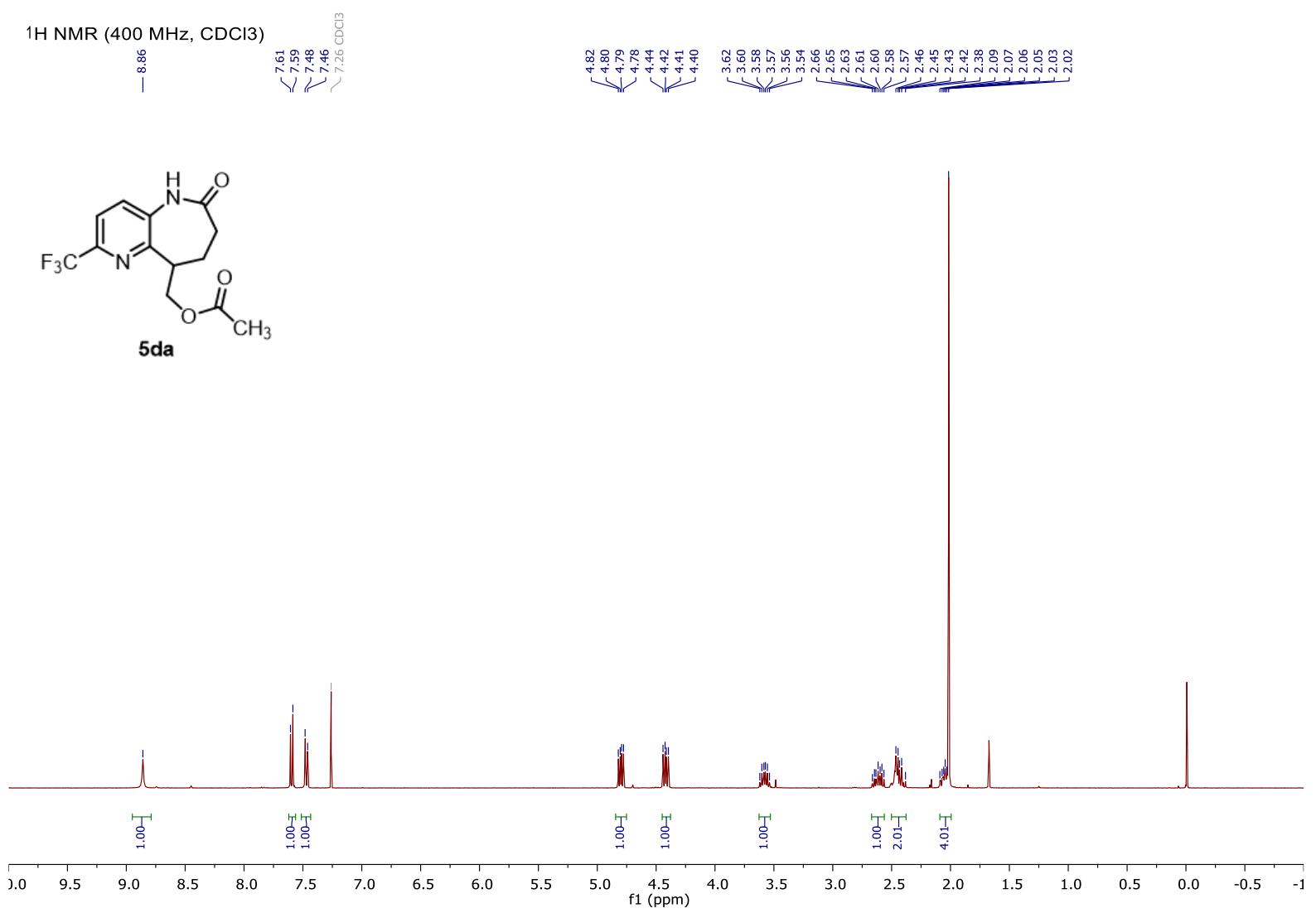

$13 \mathrm{CNMR}(101 \mathrm{MHz}, \mathrm{CDCl} 3)$

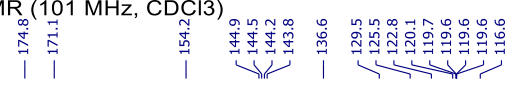<smiles>CC(=O)OC1CC(=O)Nc2ccc(C(F)(F)F)nc21</smiles>

$5 \mathrm{da}$

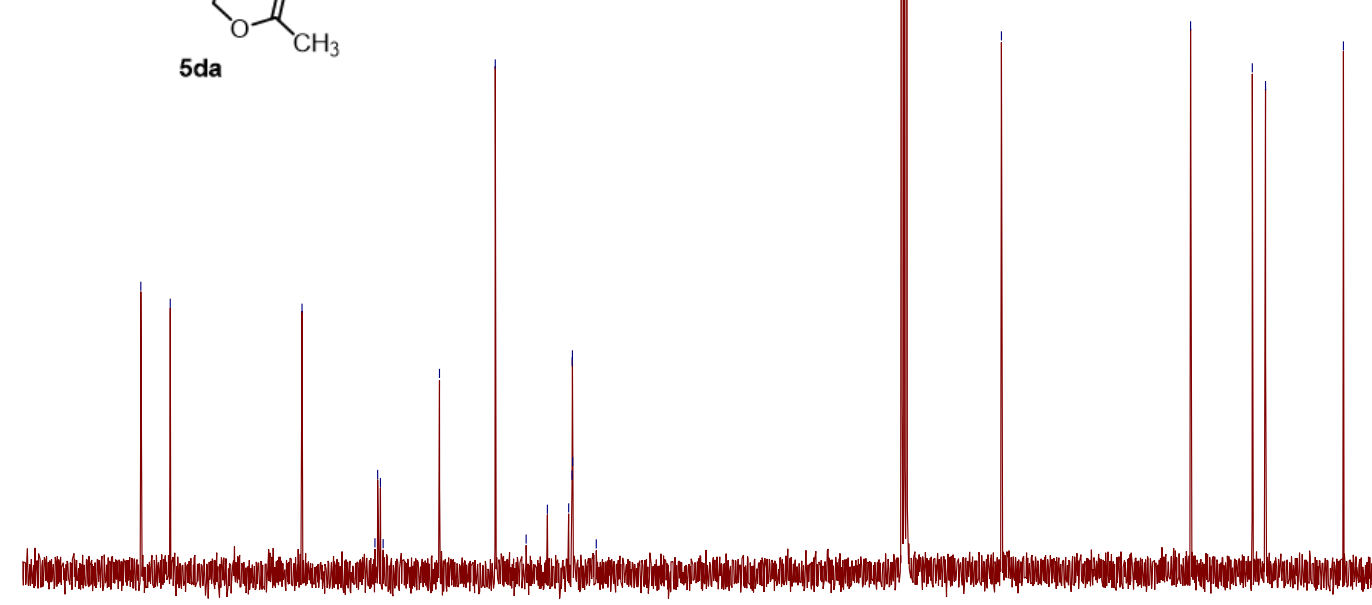

$\begin{array}{llllllllllllllllllllll} & 180 & 170 & 160 & 150 & 140 & 130 & 120 & 110 & 100 & 90 & 80 & 70 & 60 & 50 & 40 & 30 & 20 & 10 & 0 & -\therefore\end{array}$ 

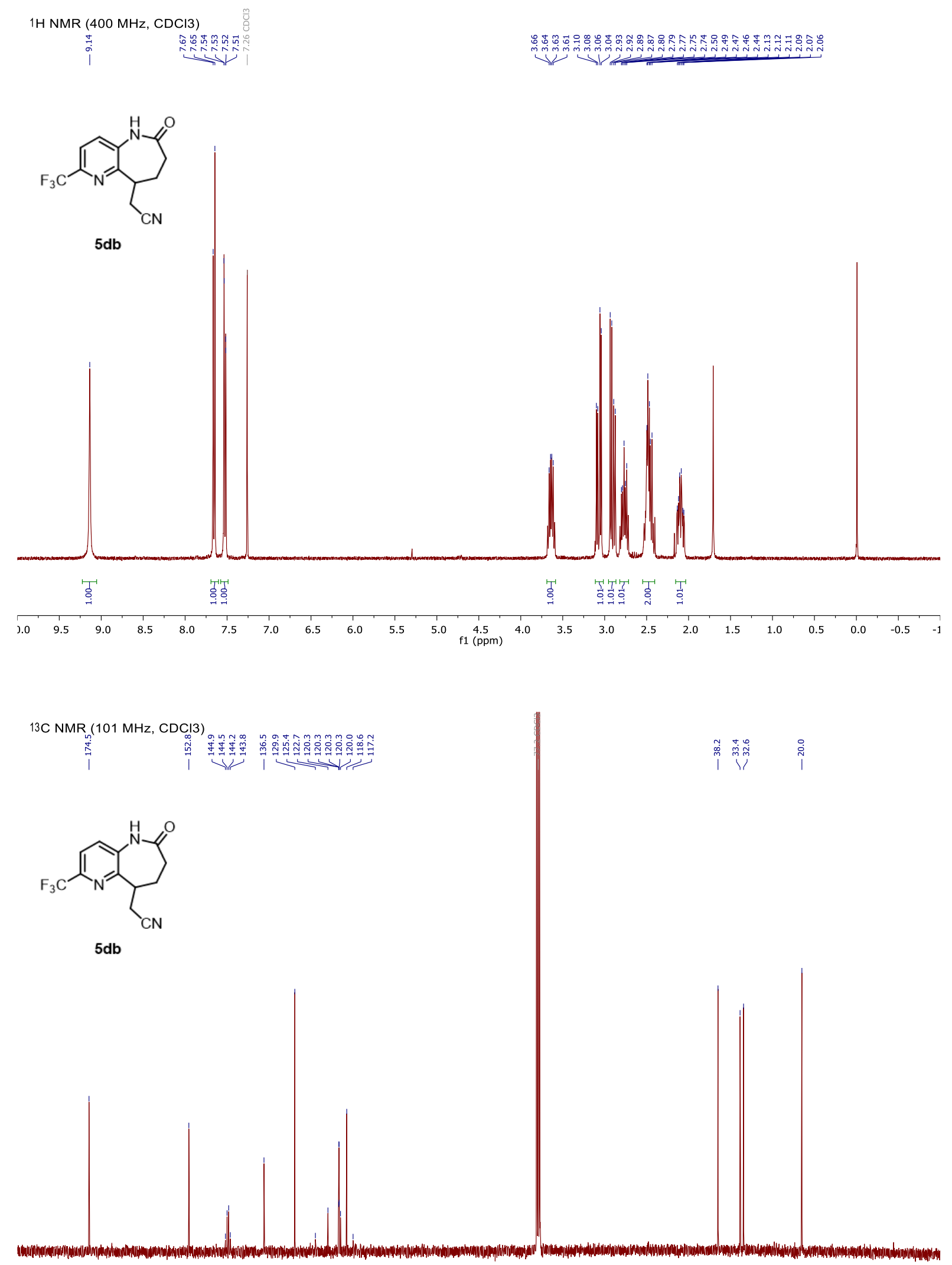

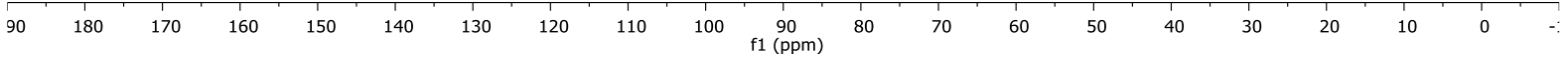


1H NMR (400 MHz, CDCl3)

素京

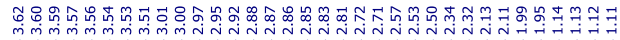<smiles>CC1CC(CC#N)c2nc(Cl)ccc2NC1=O</smiles>

5eb

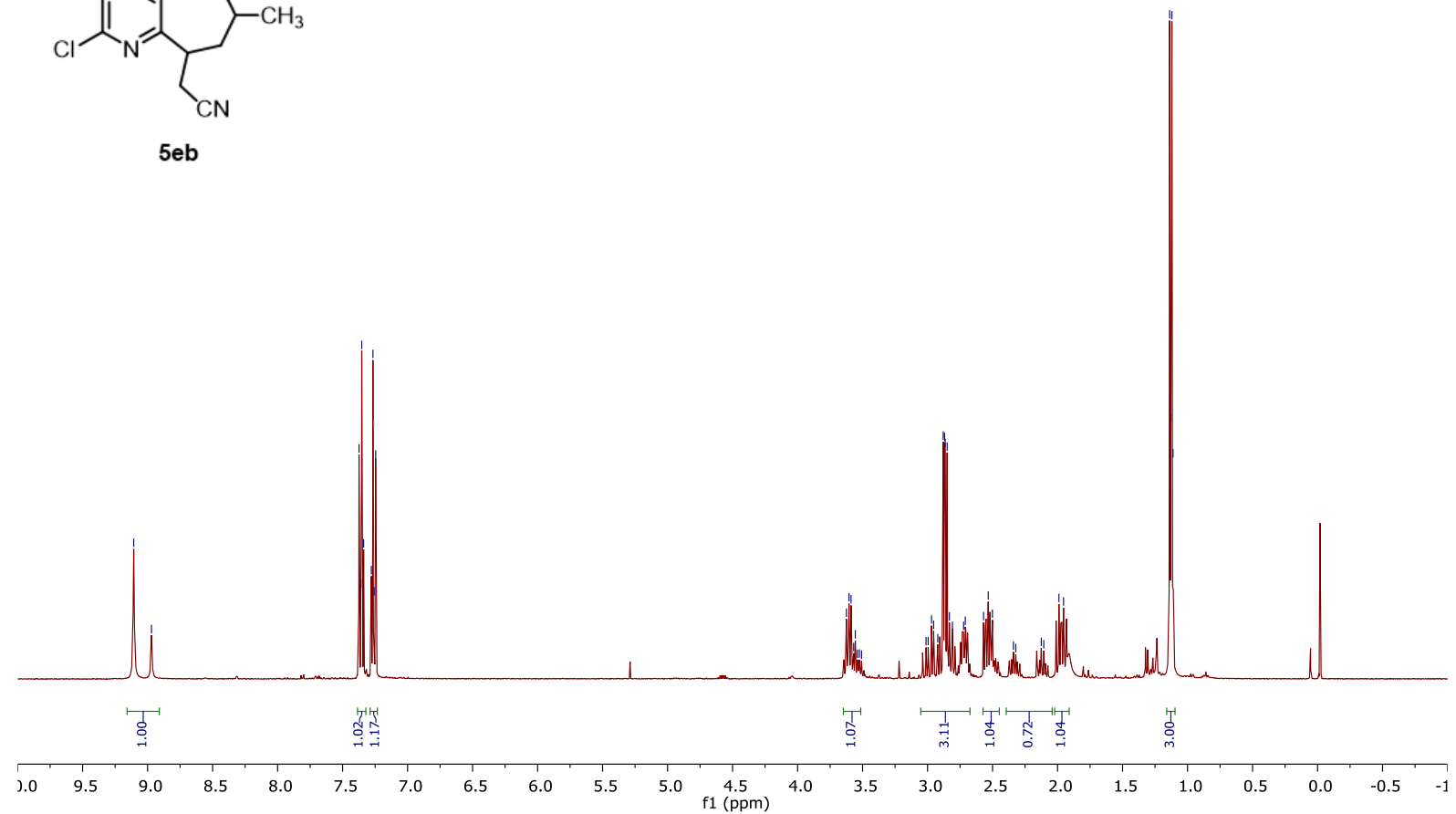

13C NMR (101 MHz, CDCl3)

至<smiles>CC1CC(CC#N)c2nc(Cl)ccc2NC1=O</smiles>

5eb

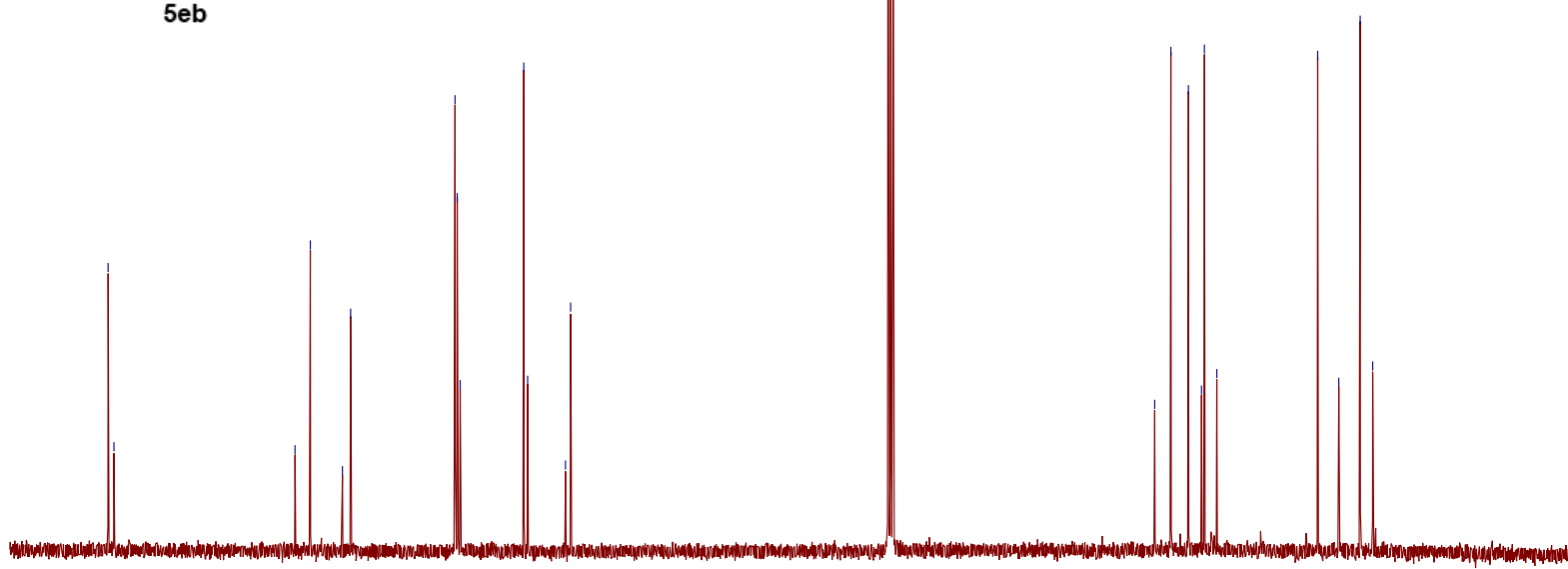

90

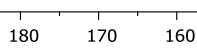

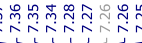



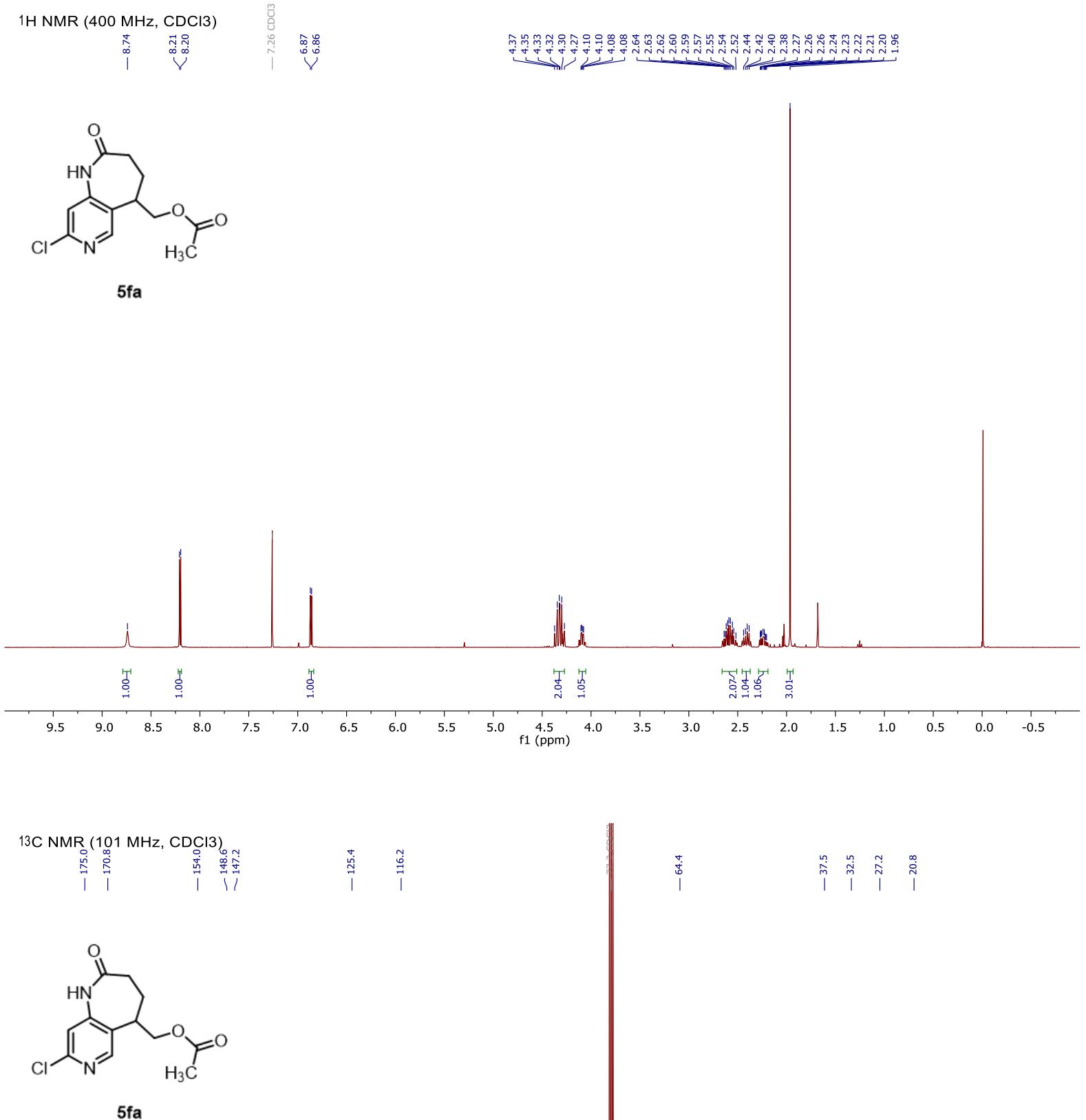

$5 f a$

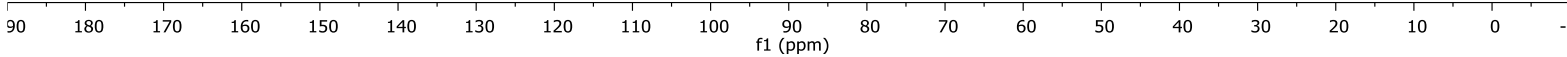




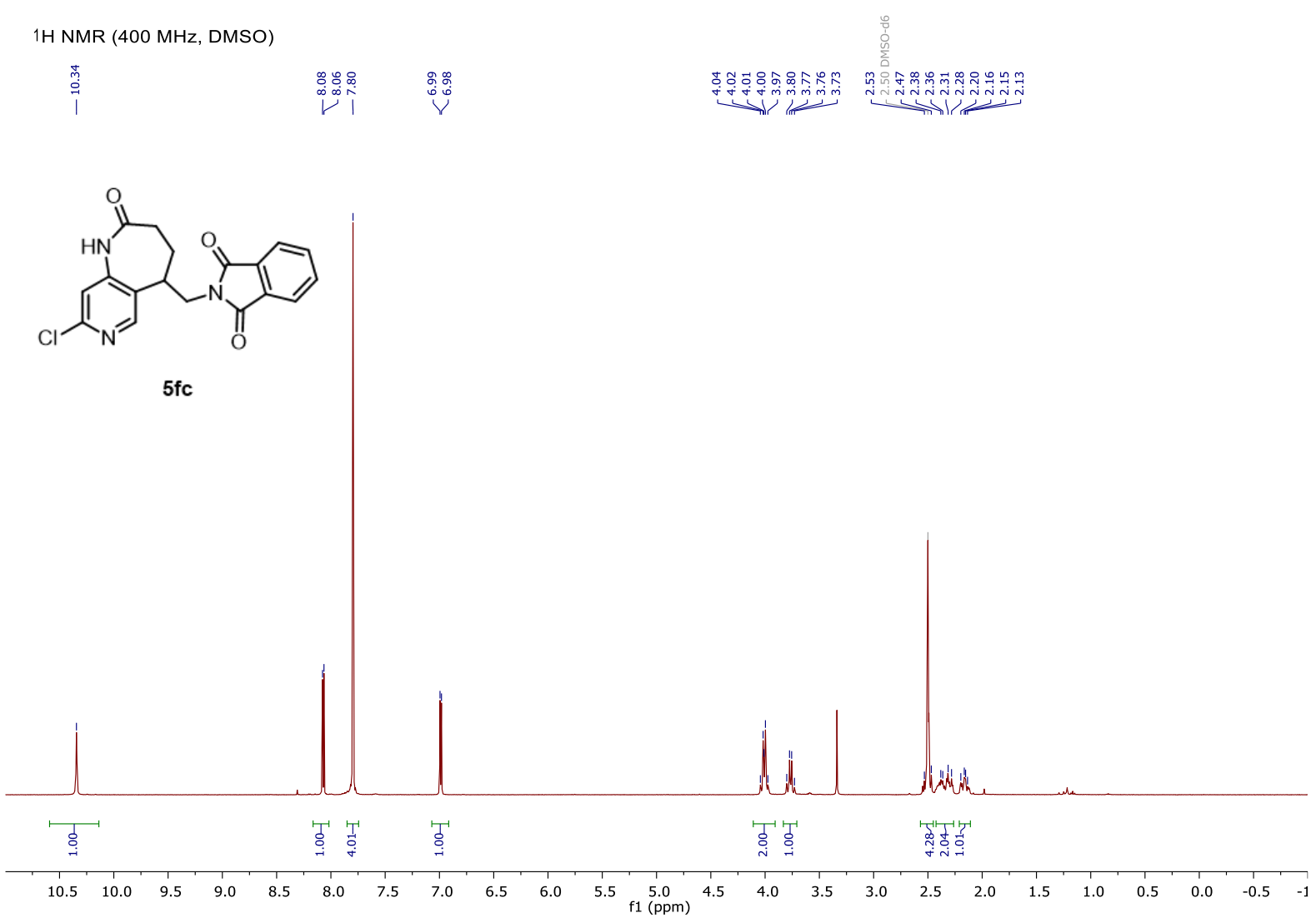

13C NMR (101 MHz, DMSO)

蕉

ำำำำำำำ

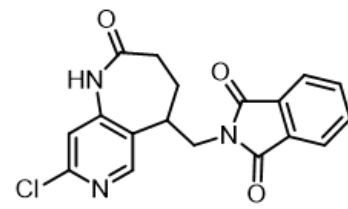

$5 \mathrm{fc}$

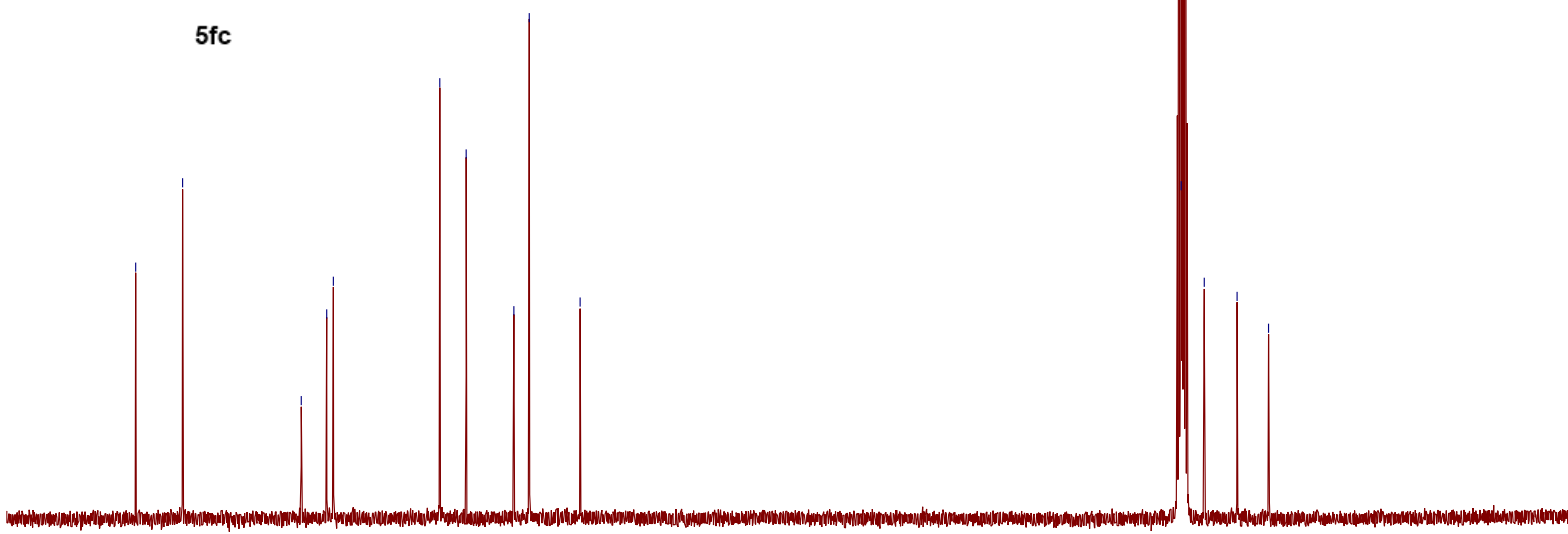

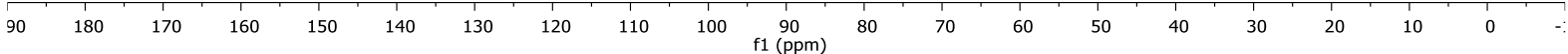



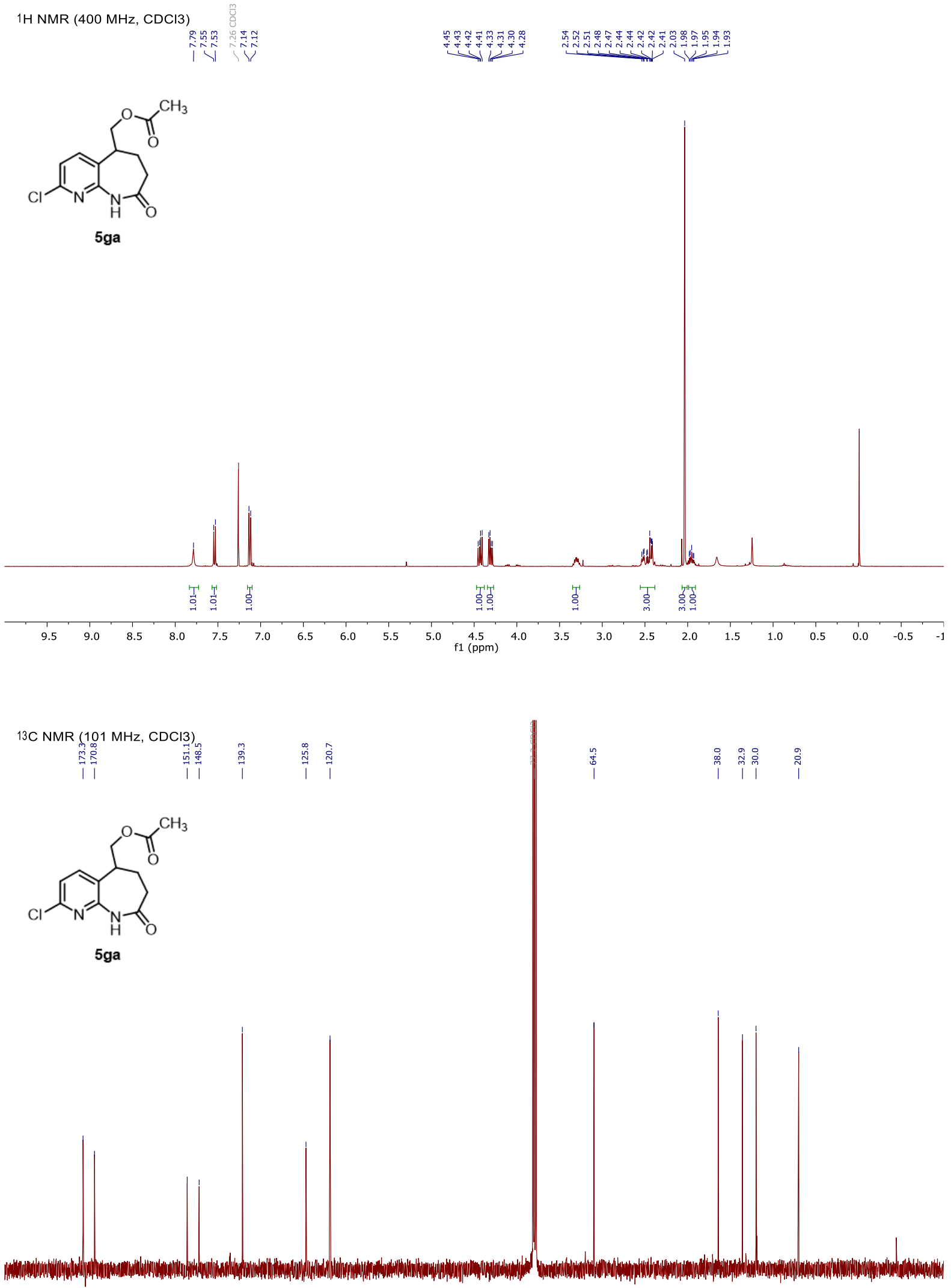

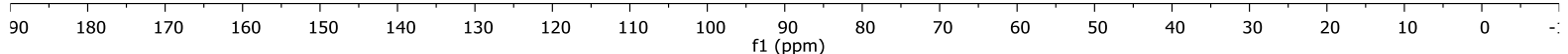




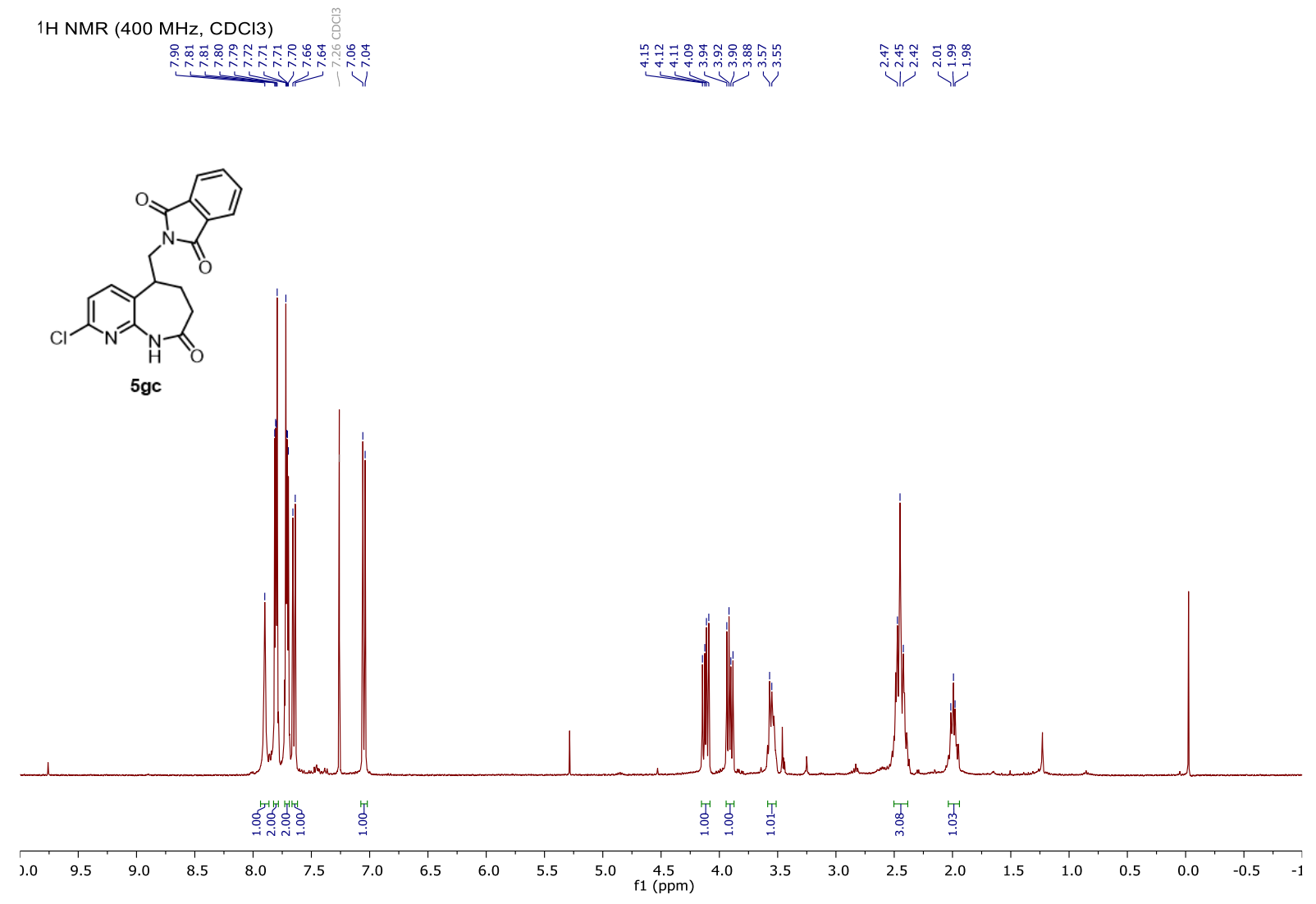

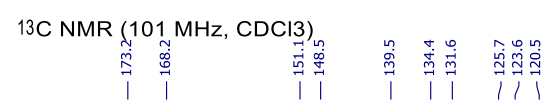
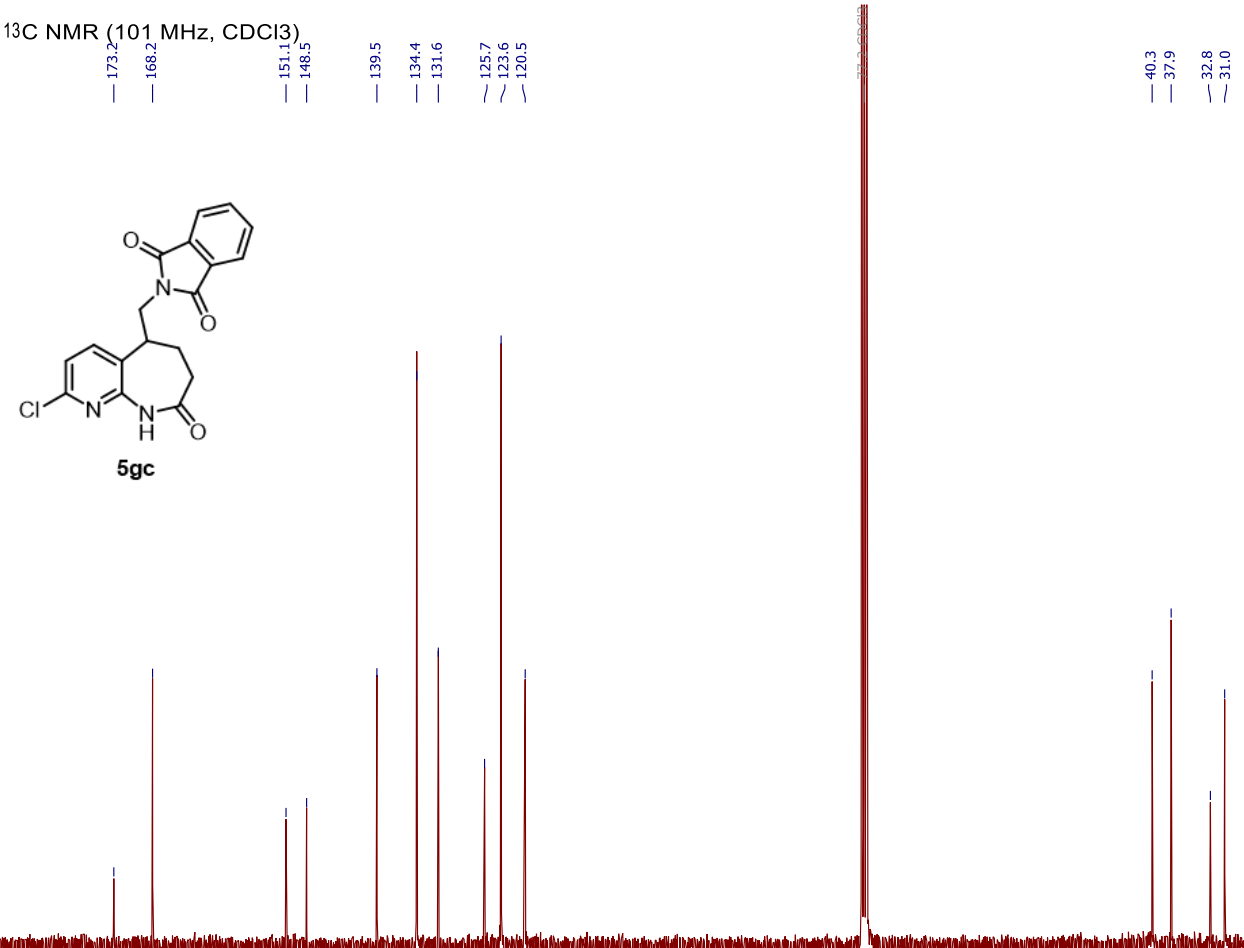

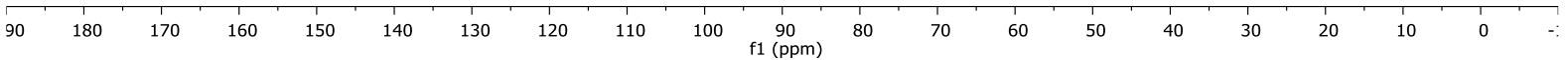


1H NMR (400 MHz, CDCl3)

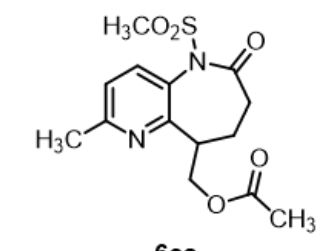

$6 c a$

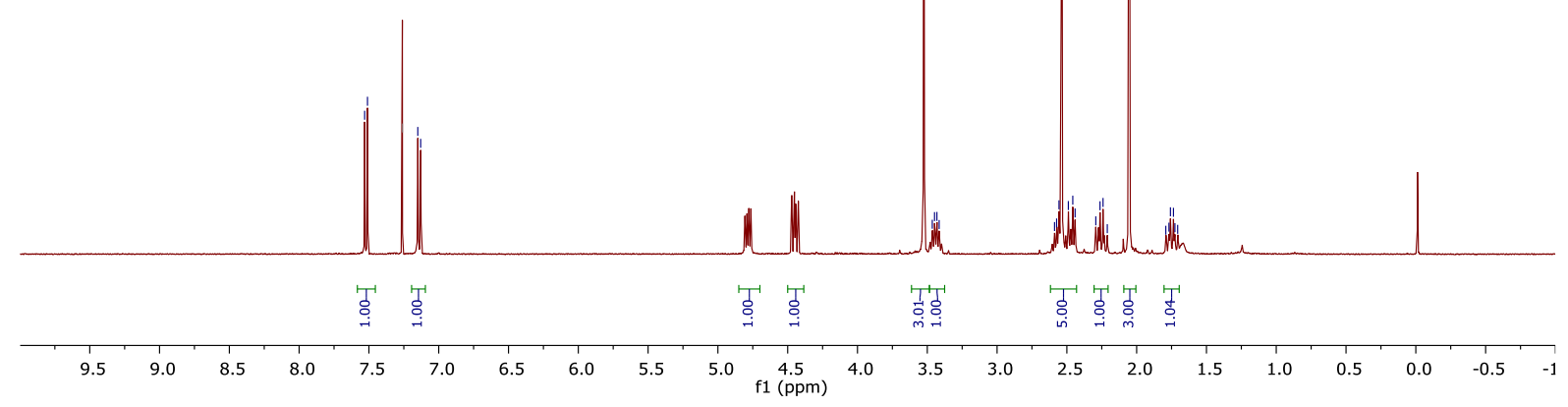

13C NMR (101 MHz, CDCl3)

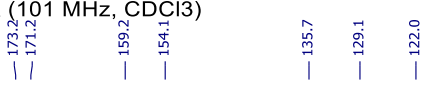

苞<smiles>CC(=O)OCC1CCC(=O)N([As](=O)O[Na])c2ccc(C)nc21</smiles>

$6 \mathrm{ca}$

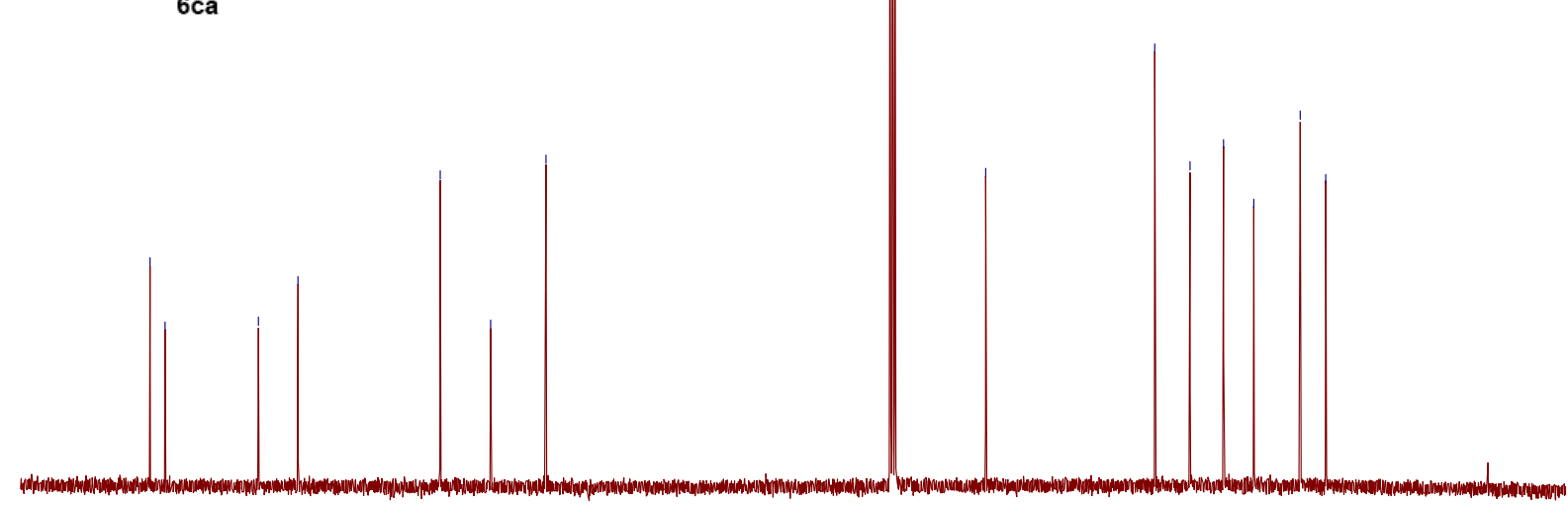

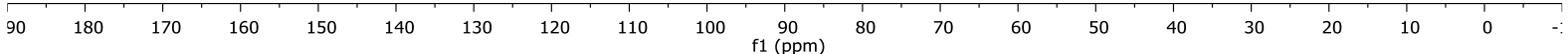



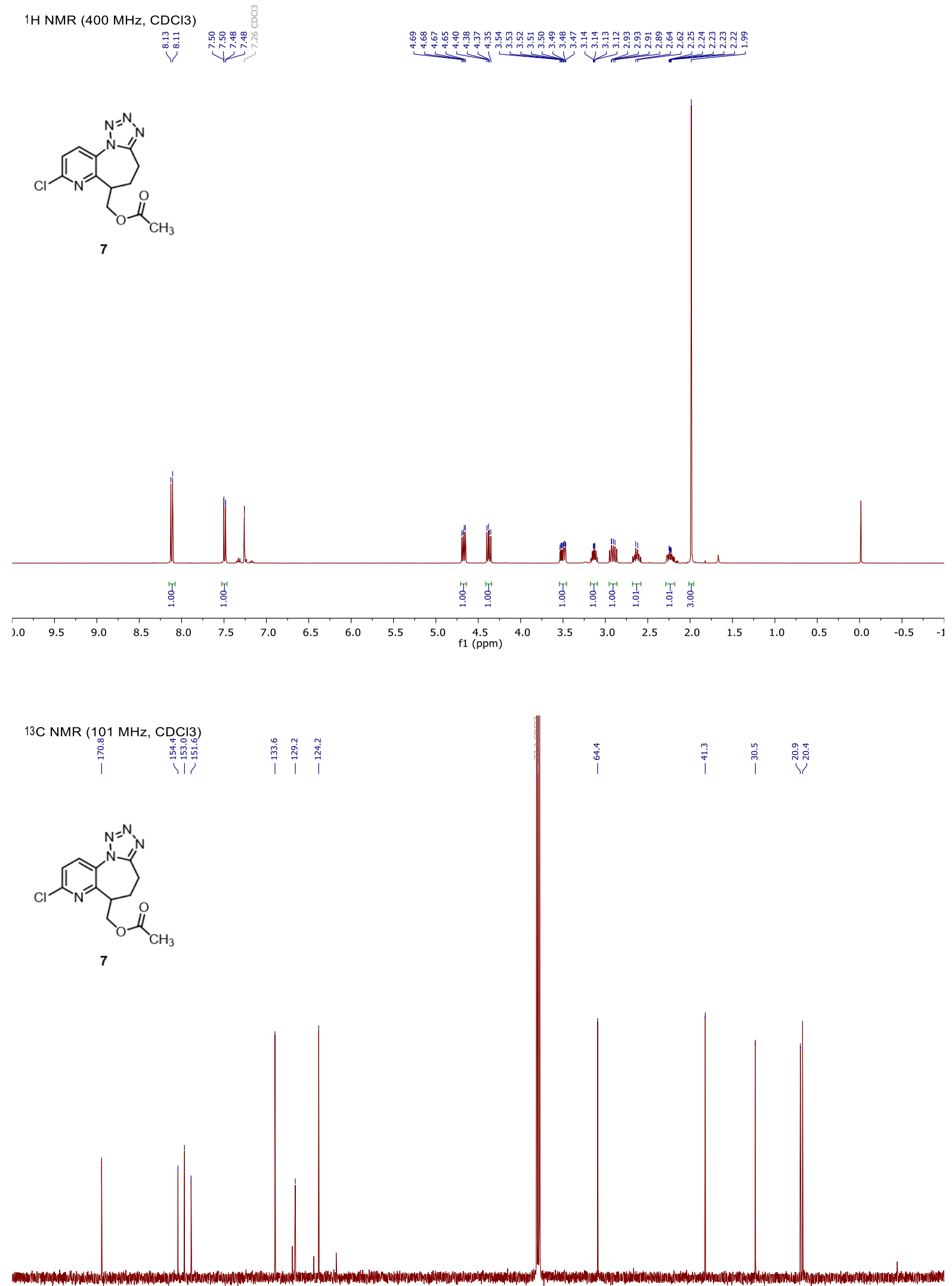

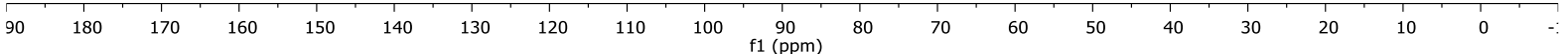



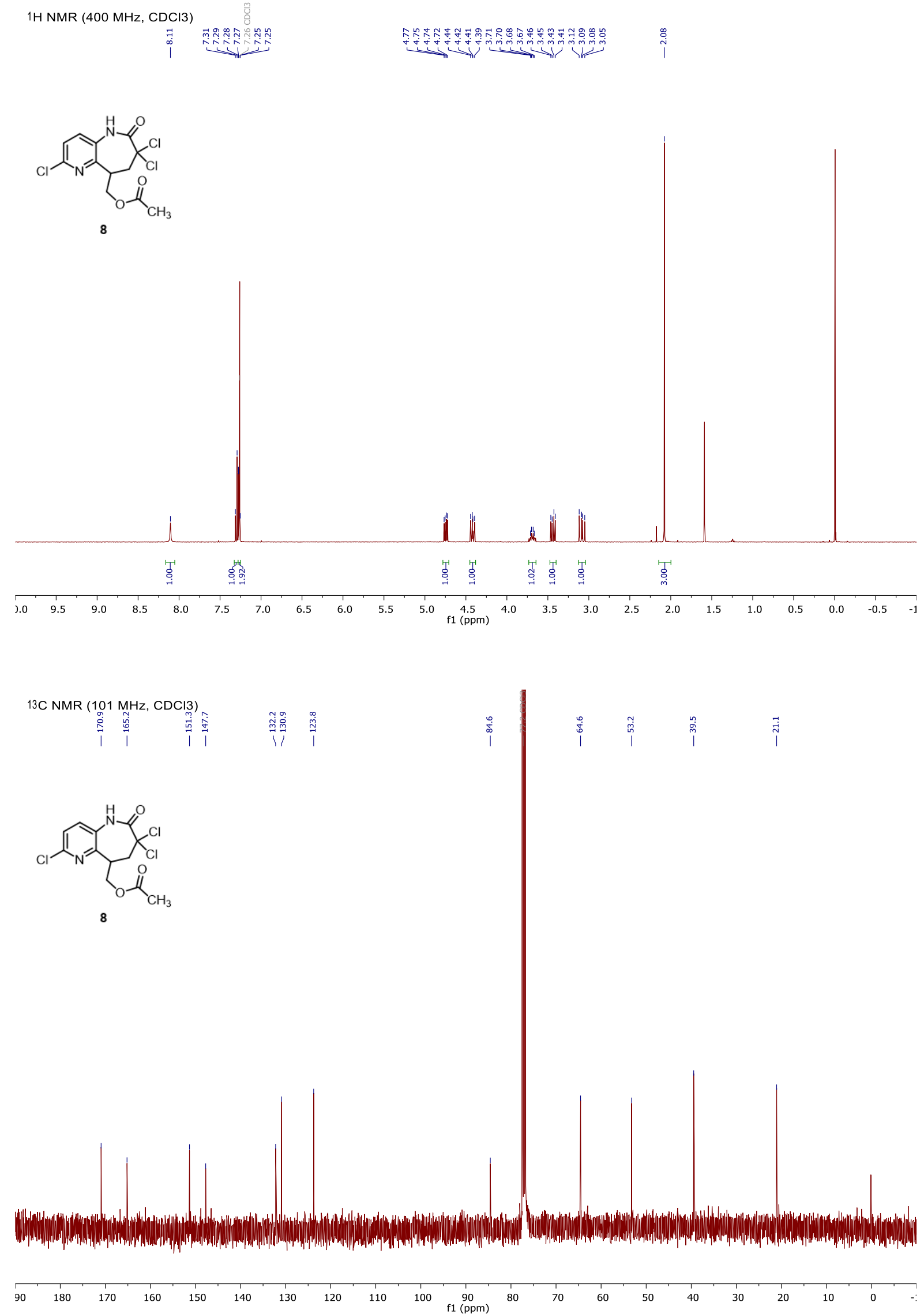

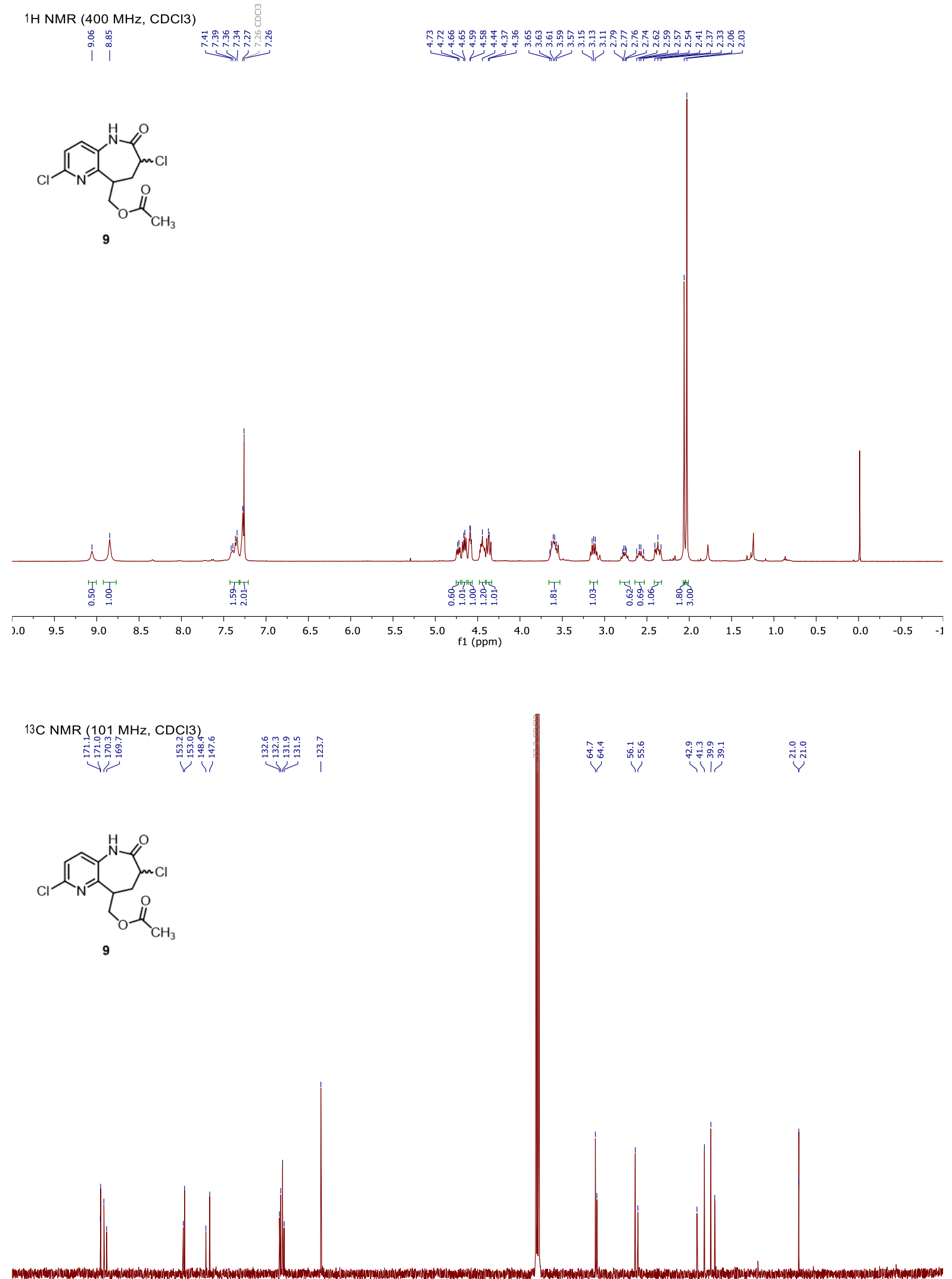

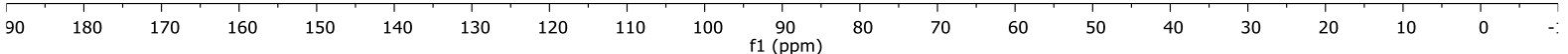

